\title{
Nanoscale Brownian Dynamics of Semiflexible Biopolymers
}

Dissertation for the award of the degree

"Doctor rerum naturalium" of the Georg-August-Universität Göttingen

within the doctoral program Physics of Biological and Complex Systems of the Göttingen Graduate School of Neurosciences, Biophysics, and Molecular Biosciences (GGNB) of the Georg-August-University School of Science (GAUSS)

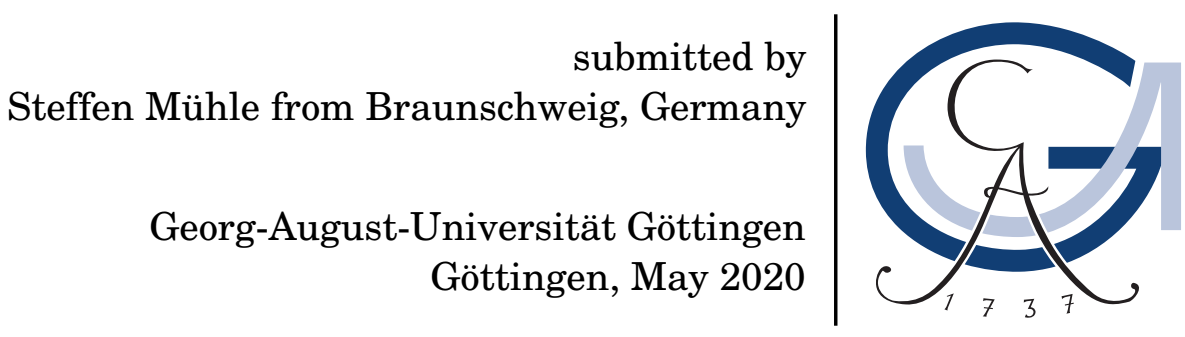




\title{
THESIS ADVISORY COMMITTEE:
}

Prof. Dr. Jörg Enderlein (1st referee)

Drittes Physikalisches Institut

Georg-August-Universität Göttingen

Prof. Dr. Max Wardetzky (2nd referee)

Institut für Numerische und Angewandte Mathematik

Georg-August-Universität Göttingen

Prof. Dr. Andreas Janshoff

Institut für Physikalische Chemie

Georg-August-Universität Göttingen

\section{FURTHER MEMBERS OF THE EXAMINATION BOARD}

\author{
Prof. Dr. Stefan Klumpp \\ Drittes Physikalisches Institut \\ Georg-August-Universität Göttingen \\ Prof. Dr. Anja Sturm \\ Institut für Mathematische Stochastik \\ Georg-August-Universität Göttingen \\ Dr. Andreas Neef \\ Campus Institut für Dynamik Biologischer Netzwerke \\ Max-Planck-Institut für Dynamik und Selbstorganisation, und \\ Georg-August Universität Göttingen
}

DATE OF THE ORAL EXAM: 16 TH JULY 2020 


\begin{abstract}
AFFIDAVIT
I hereby declare that the presented thesis has been written independently and with no other sources or aids than quoted.

Financial support by the Deutsche Forschungsgemeinschaft (DFG) via project A05 of the Collaborative Research Center SFB755 and via the Excellence Cluster 2067 "Multiscale Bioimaging" is gratefully acknowledged.
\end{abstract}

\title{
List OF RELATED PUBLICATIONS
}

Parts of this thesis have been published in the following five manuscripts:

Steffen Mühle, Man Zhou, Arindam Ghosh, Jörg Enderlein: Loop formation and translational diffusion of intrinsically disordered proteins, Physical Review E 100, 052405, doi.org/10.1103/PhysRevE.100.052405.

Published 18 November 2019.

Nora Molkenthin, Steffen Mühle, Antonia Mey, Marc Timme: Self-organized emergence of folded protein-like network structures from geometric constraints, PLOS ONE 15(2): e0229230, doi.org/10.1371/journal.pone.0229230.

Published 27 February 2020.

Rohit Satija, Dmitrii Makarov, Steffen Mühle, Atanu Das, Jörg Enderlein: Kinetics of loop closure in disordered proteins: theory vs simulations vs experiments, The Journal of Physical Chemistry B, 124, 3482-3493, doi.org/10.1021/acs.jpcb.0c01437. Published 7 April 2020.

Daja Ruhlandt, Jörg Enderlein, Steffen Mühle: Electric field lines of an arbitrarily moving point charge, American Journal of Physics 88, 5, doi.org/10.1119/10.0000189. Published 23 December 2019.

Soheil Mojiri, Sebastian Isbaner, Steffen Mühle, Hongje Jang, Albert Johann Bae, Ingo Gregor, Azam Gholami and Jörg Enderlein: Three-dimensional motion of flagella: Curvature and torsion of a chiral structure, Physical Review X.

Submitted 27 May 2020.

The topmost manuscript in particular constitutes the main project of my doctoral work. Parts of it can be found throughout this thesis (in particular section 5.2), but have been slightly altered to fit into its narrative. Similarly, parts of the other four manuscripts, as well as my contributions to an unpublished project ("Probing conformational dynamics with metal-induced energy transfer"), can be found in sections $8.1,8.4,8.3,8.2$ and 5.4 respectively. 
To my parents Berit and Werner 
The freedom to make my own mistakes was all I ever wanted. 


\begin{abstract}
Proteins are one of, if not the most, versatile of biology's tools, performing a great variety of functions in living organisms and giving rise to behavior as complex as life itself. They are linear chains of amino acids and therefore heteropolymers from a chemical point of view. Performing diverse tasks while obeying the laws of thermodynamics and microscopic physics, proteins thus invoke the interest of biologists, chemists and physicists alike.

While the theoretical foundations for describing molecular motion can be traced back more than a century, experimental techniques allowing us to measure the dynamics of single molecules have only become available in the last twenty years. Here, we aim to link the two together and use data from single-molecule experiments to infer characteristic properties of individual proteins in a systematic and quantitative manner. In particular, we have at our disposal three complementing methods which measure dynamical properties of single molecules. The first is photo-induced electron transfer paired with fluorescence correlation spectroscopy (PET-FCS) which measures their end-to-end or internal-to-end loop formation rate, the second is dual-focus fluorescence correlation spectroscopy (2fFCS) which measures their hydrodynamic radius, and the third is dynamic metal-induced energy transfer (dynaMIET) which measures their reconfiguration time when bound to a surface.

In this thesis, we develop a polymer model which enables the efficient interpretation of results from PET-FCS, 2fFCS and dynaMIET experiments. This model is a bead-rod chain which takes into account hydrodynamic interactions, excluded volume effects, bending rigidity and the fluorophore with which the protein is labeled, while including only two free parameters. It quantitatively reproduces systematically measured data from PET-FCS and 2fFCS applied to glycine-serine (GS) repeats - the prototype of an intrinsically disordered protein (IDP). Loop formation dynamics in GS-repeats typically take tens of nanoseconds, and their hydrodynamic radius is around one nanometer. From this data, the model yields a persistence length of $l_{P}=5.2 \pm 1.9 \AA$ and one amino acid's hydrodynamic radius of $a=3.5 \pm 0.7 \AA$, while at the same time validating the important role of excluded volume effects in the dynamics of GS-repeats.

Thus, we now have at hand a combined method of performing single-molecule experiments and Brownian dynamics simulations for IDPs which yields quantitative insights into their molecular properties. This enables further study of the elastic and hydrodynamic properties associated with different amino acid sequences. Extending the model to take into account secondary structures, inhomogeneities or internal friction effects should be subject to future work. This may improve our understanding of the mechanisms governing protein function and folding, and hence contribute to novel medical treatments and drug design.
\end{abstract}




\section{TABLe OF Contents}

$\begin{array}{ll}\text { Conventions and theorems } & 1\end{array}$

List of symbols and acronyms 2

1 Introduction $\quad 3$

2 Theory 15

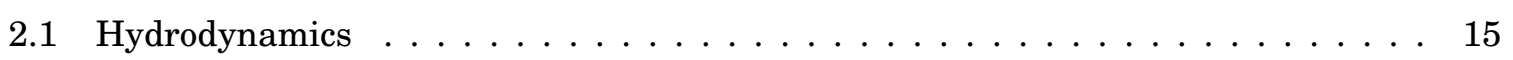

2.1 .1 Continuity equation $\ldots \ldots \ldots \ldots \ldots \ldots \ldots$

2.1 .2 Navier-Stokes equation . . . . . . . . . . . . . . . . . 16

2.1 .3 Stokes equation . . . . . . . . . . . . . . . . . . . 17

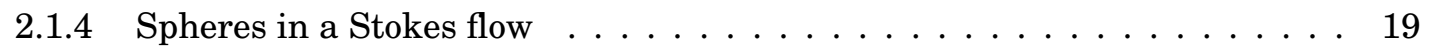

2.1.5 Rotne-Prager approximation . . . . . . . . . . . . . . . . . 22

2.1.6 Hydrodynamics near a wall . . . . . . . . . . . . . . . . . . 25

2.2 Statistical mechanics . . . . . . . . . . . . . . . . . . . . . 27

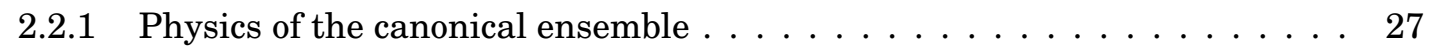

2.2.2 Basic notions of probability theory . . . . . . . . . . . . . 32

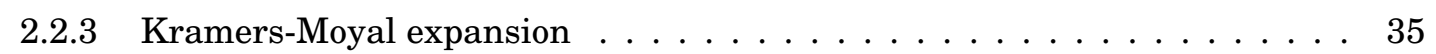

2.2.4 The Fokker-Planck equation $\ldots \ldots \ldots \ldots \ldots \ldots$

2.2 .5 Langevin equation . . . . . . . . . . . . . . . . . . 41

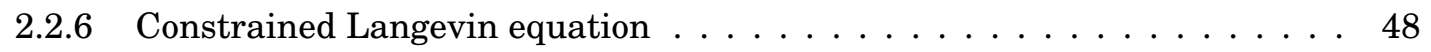

2.2.7 Diffusion coefficient and hydrodynamic radius . . . . . . . . . . . . 52

2.3 Differential geometry . . . . . . . . . . . . . . . . . . . . 54

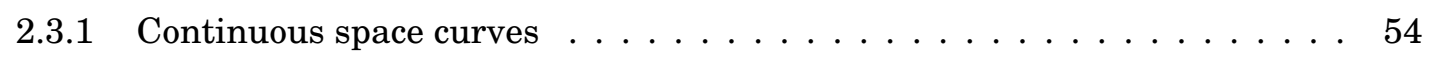

2.3 .2 Discrete space curves $\ldots \ldots \ldots \ldots \ldots \ldots$

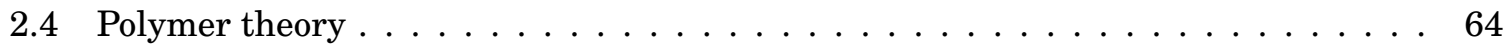

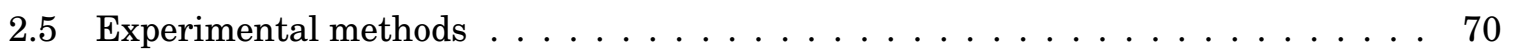

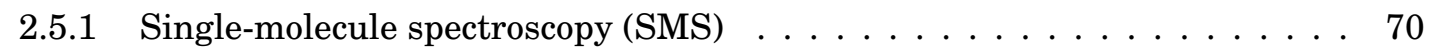

2.5.2 Fluorescence correlation spectroscopy (FCS) . . . . . . . . . . . . 70

2.5.3 Photo-induced electron transfer $($ PET) . . . . . . . . . . . . . . . . . . . . 74

2.5.4 Dynamic metal-induced energy transfer (dynaMIET) . . . . . . . . . . 76

2.5.5 Dual-focus fluorescence correlation spectroscopy (2fFCS) . . . . . . . . . 79 
4 Numerical methods $\quad 85$

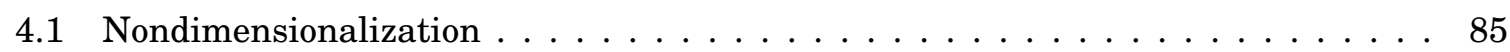

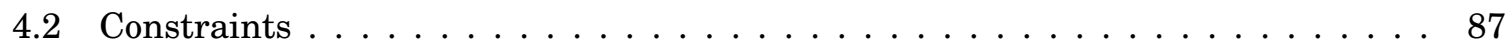

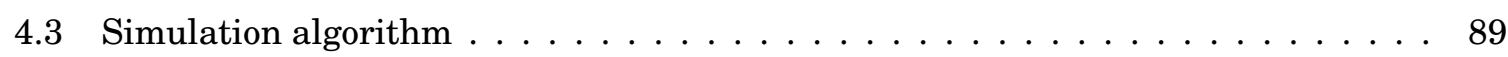

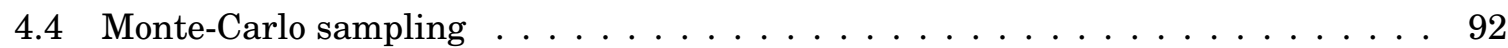

4.5 Hydrodynamic radius of a polymer chain $\ldots \ldots \ldots \ldots \ldots \ldots$

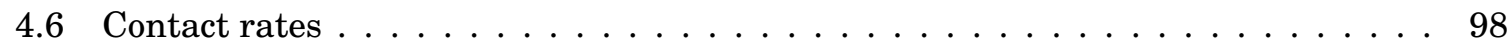

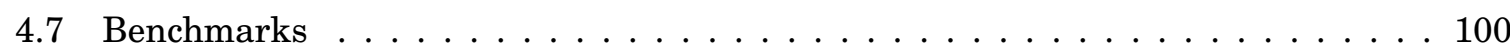

5 Applications $\quad 107$

5.1 Model behavior . . . . . . . . . . . . . . . . . . . . . . . . . 107

5.1 .1 Hydrodynamic radius . . . . . . . . . . . . . . . . . 107

5.1 .2 Contact rate . . . . . . . . . . . . . . . . . . . . . . . 111

5.2 End-to-end contact rates and hydrodynamic radii of GS-repeats . . . . . . . . . 115

5.3 Internal-to-end contact rates and hydrodynamic radii of FG-proteins . . . . . . . . 129

5.4 Polymer dynamics using dynaMIET . . . . . . . . . . . . . . . . . . . . . 133

6 Discussion and conclusion 141

7 Outlook $\quad 151$

7.1 Dynamics in generalized coordinates $\ldots \ldots \ldots \ldots$. . . . . . . . . . 151

7.2 Investigating dynaMIET . . . . . . . . . . . . . . . . . . . . . . 153

7.3 Internal friction . . . . . . . . . . . . . . . . . . . . . . 153

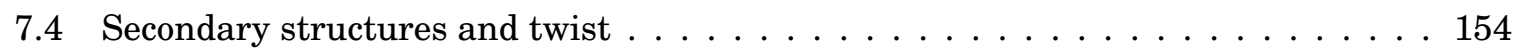

7.5 Repeat current protocol . . . . . . . . . . . . . . . . . . . 156

8 Other contributions $\quad 157$

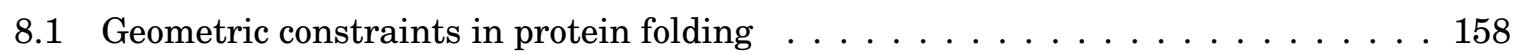

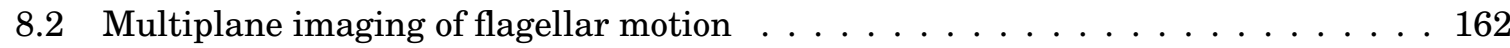

8.3 Electric field lines of an arbitrarily moving point charge . . . . . . . . . . . . 167

8.4 Kinetics of loop closure in disordered proteins . . . . . . . . . . . . . . . . 171 
A Convergence of discrete curvatures to the worm-like chain

$\begin{array}{ll}\text { B Pawula's theorem } & 177\end{array}$

$\begin{array}{ll}\text { C Entropic pseudoforce } & 179\end{array}$

D Angular correlations in worm-like chains 183

$\begin{array}{ll}\text { E Solving constraints } & 185\end{array}$

$\begin{array}{lll}\text { F Diffusion coefficient - benchmarks } & 189\end{array}$

$\begin{array}{lll}\text { G Secondary structures and twist elasticity } & 197\end{array}$

$\begin{array}{ll}\text { Bibliography } & 201\end{array}$

$\begin{array}{ll}\text { Acknowledgements } & 215\end{array}$ 


\section{CONVENTIONS AND THEOREMS}

Conventions Any simple symbol such as $x, N$ or $\lambda$ denotes a number, regardless of whether it is real, imaginary or complex. Column vectors are denoted as $\vec{x}$, row vectors as $\vec{x}^{T}$ and matrices as bold letters such as $\mathbf{D}$. The unity matrix in $N$ dimensions is denoted by $\mathbb{1}_{N \times N}$. Scalar elements of vectors and matrices are written as $x_{i}$ and $D_{i j}$. Submatrices (blocks) of a matrix $\mathbf{D}$ are written as $\mathbf{D}_{i j}$. The matrix product is $\mathbf{A} \cdot \mathbf{B}$ which is also used for $1 \times 1$-matrices, i.e. scalar. This means $\vec{x}^{T} \cdot \vec{x}$ is a scalar and $\vec{x} \cdot \vec{x}^{T}$ is a matrix. Occasionally, we also write $\vec{x} \cdot \vec{x}$ for readability, by which we mean the dot product $\vec{x}^{T} \cdot \vec{x}$. Values of functions are denoted with round parenthesis such as $f(t)$ and functionals as capital letters with square brackets such as $G[f]$ (capital letters are not necessarily functionals, though). The partial derivative of $f$ with respect to $x$ is $\partial_{x} f:=\partial f / \partial x$, and the total derivative of $f$ with respect to $x$ is $\mathrm{d}_{x} f:=\mathrm{d} f / \mathrm{d} x$. When $f$ depends on both spatial coordinates $\vec{x}=\left(x_{1}, \ldots, x_{n}\right)^{T}$ and time $t$, we write $f(\vec{x}, t)$ and $\partial_{k} f=\partial f / \partial x_{k}$ but still $\partial_{t} f=\partial f / \partial t$, so the letter $t$ has special meaning in this context. The nabla operator is $\vec{\nabla}=\left(\partial_{1}, \ldots, \partial_{n}\right)^{T}$ such that $\vec{\nabla} \cdot f$ is the gradient of $f, \vec{\nabla} \cdot \vec{g}$ is the divergence of $\vec{g}$ and $\vec{\nabla} \times \vec{g}$ is its curl. We further use Einstein's index convention which states that any index appearing twice in a term is summed over, such that e.g. $a_{i} b_{i} c_{j}=\sum_{i} a_{i} b_{i} c_{j}$. The Dirac delta function is written as $\delta(t-s)$, and the Kronecker delta as $\delta_{i j}$. Pointed brackets $\langle\cdot\rangle$ denote the ensemble or time average of a stochastic quantity. The mean value of a random variable is denoted by $\mu$ and its variance by $\sigma^{2}$, but $\mu$ and $\sigma$ also have other meanings, and the reader is expected to distinguish between them. Furthermore, we set Boltzmann's constant to $k_{B}=1$. As a consequence, the entropy $S$ is dimensionless, and the temperature $T$ is an energy.

Theorems The reader is assumed to be familiar with basic calculus, stochastics and algebra, and in particular with Gauss' integral theorem and L'Hospital's theorem concerning limits of fractions. Furthermore, we utilize a version of Reynold's transport theorem which states that the total time derivative of an integral over a volume $V$ can be pulled into the integral, thereby becoming a partial derivative

$$
\mathrm{d}_{t} \int_{V} f(\vec{x}) \mathrm{d} \vec{x}=\int_{V} \partial_{t} f(\vec{x}) \mathrm{d} \vec{x}
$$

which holds true when $V$ is constant over time. Moreover, we make use of the central limit theorem. It states that the sum of $N$ identical, independent stochastic variables with mean values $\mu$ and variances $\sigma^{2}$ is a new stochastic variable with the following properties; its mean and variance are $N \cdot \mu$ and $N \cdot \sigma^{2}$ (which is trivial), and as $N$ goes to infinity, its probability distribution becomes a Gaussian, regardless of the probability distribution of the stochastic variables which are summed over (which is fascinating). More precise formulations and proofs of these theorems can be found in numerous textbooks such as references [1, 2]. 


\section{LIST OF SYMBOLS AND ACRONYMS}

\begin{tabular}{|c|c|c|c|c|c|}
\hline symbol & name & see page & symbol & name & see page \\
\hline $2 \mathrm{fFCS}$ & dual-focal fluorescence & 79 & $l_{P}$ & persistence length & 67 \\
\hline & correlation spectroscopy & & $\vec{m}_{i}$ & material frame & 55,59 \\
\hline$a$ & hydrodynamic bead radius & 19,82 & $\mathrm{MC}$ & (Metropolis) Monte-Carlo & 92 \\
\hline$\alpha^{(n)}$ & jump moment & 35 & $\mu$ & mobility matrix & 22 \\
\hline$\vec{b}_{i}$ & Bishop frame & 55,60 & $N$ & number of beads & 22 \\
\hline$\beta$ & inverse temperature & 29 & $\Omega$ & phase space / rotation rate & 27,54 \\
\hline $\mathbf{B}$ & Cholesky decomp. of $\mathbf{D}$ & 47 & $p$ & pressure / probability & 17,28 \\
\hline$d$ & bond length & 81 & PET & photo-induced & 74 \\
\hline$D$ & diffusion coefficient & 52 & & electron transfer & \\
\hline $\mathbf{D}$ & diffusion matrix & 24,83 & $\Psi$ & complex curvature & 56 \\
\hline $\mathbf{D}_{i j}$ & $3 \times 3$ block in $\mathbf{D}$ & 24,83 & $\vec{r}$ & Cartesian position vector & 19 \\
\hline dynaMIET & dynamic metal-induced & 76 & $R_{C}$ & critical contact radius & 76 \\
\hline & energy transfer & & $\mathrm{Re}$ & Reynolds number & 17 \\
\hline$E$ & energy & $27,57,62$ & $R_{\mathrm{EV}}$ & excluded volume radius & 81 \\
\hline$\epsilon$ & power law exponent & 115 & $R_{H}$ & hydrodynamic radius & 53 \\
\hline$\eta$ & viscosity & 17 & $\rho$ & mass density & 15 \\
\hline$\vec{f}$ & force density & 18 & $s$ & arc length & 54 \\
\hline$\vec{F}$ & force & 19 & $S$ & entropy & 28 \\
\hline$F$ & free energy & 30 & $S_{C}$ & constraint entropy & 49 \\
\hline FCS & fluorescence correlation & 70 & $\sigma$ & bending stiffness (length) & 68 \\
\hline & spectroscopy & & $t$ & time & 16 \\
\hline$g$ & constraint & 50,87 & $t_{0}$ & numerical time scale & 87 \\
\hline $\mathbf{G}$ & constraint matrix & 50 & $\vec{t}$ & tangent vector & 54,59 \\
\hline$\gamma$ & friction coefficient & 19 & $T$ & temperature & 29 \\
\hline$\vec{j}$ & flux / current & $16,32,37$ & $\mathbf{T}$ & Oseen matrix & 18 \\
\hline$\kappa$ & curvature & 56,60 & $\theta$ & bending angle & 60 \\
\hline$k$ & transition rate & 32,34 & $\vec{v}$ & velocity & 15,19 \\
\hline$k_{-}$ & opening rate & 74 & $W$ & Wiener process & 42 \\
\hline$k_{+}$ & closing (contact) rate & 74 & $\xi$ & white noise & 41 \\
\hline$L$ & contour length & 54,117 & $Z$ & partition function & 29 \\
\hline$\lambda$ & Lagrangian multiplier & 48,51 & $\zeta$ & tail effect & 114,116 \\
\hline
\end{tabular}




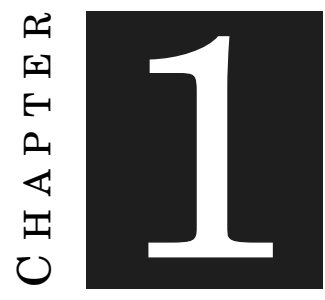

\title{
INTRODUCTION
}

\begin{abstract}
A $t$ least since Isaac Newton has discovered the link between differential equations and the movement of physical objects, we humans can use mathematics to predict the future. While these predictions can sometimes only be approximate or statistical due to practical or fundamental limitations, the remarkable fact remains: Using abstract mathematical rules, seemingly only concerning the manipulation of symbols on a piece of paper, we can make logical leaps exceeding our mind's limitations, and find out how the world around us works.

Consider for instance two pendulums connected by a spring, swinging with small amplitudes. Personally, I have a good intuition about how gravitational restoring forces lead to period motion of the pendulums, and how they push and pull at each other, but must admit that this intuition does not suffice for quantitative reasoning. Without mathematics, how could I figure out that the pendulums' motions are precisely described by the sum and difference of two sine functions, regardless of their initial speed and deflection? Perhaps a rare genius like Leonhard Euler could have done that, but nowadays anyone can do so by absent-mindedly following the laws governing differential equations, matrices and determinants - even without having any physical intuition whatsoever. With similar ease, one finds that one harmonic oscillation of an apple with mass $m$ connected to a spring with stiffness $k$ lasts exactly $2 \pi \sqrt{m / k}$. I find this fascinating: we have at hand a concrete numerical value which is entirely independent of how far the apple was initially pulled from the spring's equilibrium position. It is valid for any values of $m$ and $k$, and, of course, is by no means limited to apples. All this can be tediously tested in numerous real-world experiments, but is almost trivial to prove with a few lines of calculus. Furthermore, this insight implies that the value of $\pi$ can be measured with a rope, a stone and a stop watch.
\end{abstract}


As a physics student, one learns to apply this sort of trick in a number of well-understood situations: planets circling each other, electric circuits, quantum particles in a box, electromagnetic fields around point charges, an ideal gas and so forth. The methods of theoretical physics are by no means limited to such toy systems, however. After all, why should the laws of physics be applicable in laboratories and galaxies, but not inside our cells and brains? With the developments in statistical physics and thanks to an ever expanding amount of numerical modeling techniques and computational power at our disposal, biological systems have increasingly shifted into the focus of modern physics. While the study of fundamental physics has already made - mostly as a side effect - remarkable impacts on the technological advancement of humankind (navigational devices such as GPS, engines for the industrial revolution, transistors for electronic devices, X-ray and NMR scans for medicine, not to speak of nuclear plants and bombs), the importance of understanding biological systems cannot be overestimated. Without a doubt, figuring out the physical mechanisms which make a living organism function is of great academical and philosophical importance, and we cannot begin to imagine the impact such understanding may have on future technological and medical applications.

One of the first steps in this direction is to study the basic building blocks of biology such as proteins. Naturally, their properties are of interest in their biological environment, i.e. dissolved in water. From a physicist's point of view, this solution of water and proteins is described as the canonical ensemble: the system of interest is coupled to a heat bath of a given temperature with which it can exchange heat, and thus constantly undergoes thermal fluctuations. The physical disciplines treating the canonical ensemble are thermodynamics and statistical physics. They are concerned with complex systems consisting of particles which are too numerous to realistically be treated non-statistically. Classical thermodynamics is incredibly powerful in that it immediately delivers all statistical properties of the system (e.g. protein) from its energy function alone; they are given by the Boltzmann distribution for molecular conformations. However, thermodynamics does not include (or require) any notion of time which is its power and flaw at the same time. How fast does a protein change its conformations? How frequently do two sites on the molecule come together and form a loop? These questions are of great biological and physical interest, and classical thermodynamics explicitly does not answer them.

Since it is reasonable to say that this entire thesis is concerned with dynamical properties of polymer chains in thermal equilibrium, let us clarify the distinction between statistics and dynamics with an analogy: Imagine $x$ to be a conformation of a polymer chain, and we have at hand $10^{6}$ independently taken pictures of that chain. It is then easy to extract a probability distribution for $x$ from these snapshots and determine e.g. its average end-to-end distance - a statistical property. In order to determine dynamical properties, we also require knowledge about the order of the pictures, and the time which has passed between them. 
About proteins Proteins are biology's incredibly versatile tool to perform a diverse array of functions in living organisms. For instance, the dry mass of Escherichia coli (E. coli) bacteria consists of about $50 \%$ proteins. While the human body consists of around $60 \%$ water, proteins still make up around $17 \%$ of our weight - almost half of our dry mass. Chemically speaking, each protein is a linear chain of subunits which are called amino acids. From a theorist's perspective, a protein consisting of $N$ amino acids $A_{i}$ is thus simply the linear chain $A_{1}-A_{2}-A_{3}-\cdots-A_{N}$, which is called its primary structure. There are twenty natural different amino acids in the human body, each of which consists of a backbone and a side chain. The simplified two-dimensional chemical structure of an amino acid is<smiles>[R]C(N)C(=O)O</smiles>

where the central N-C-C chain is called its backbone, and "R" stands for the amino acid's side chain. The side chain is unique for all twenty amino acids but always branches out from the central carbon atom $\left(C_{\alpha}\right)$. Two amino acids can bond together by forming a peptide bond: the left part $\left(\mathrm{NH}_{2}\right)$ of the first amino acid reacts with the right part $(\mathrm{OH})$ of the second. The result is that a water molecule $\left(\mathrm{H}_{2} \mathrm{O}\right)$ is emitted and the reaction product (a protein) has a combined backbone which is N-C-C-N-C-C, and two side chains $R_{1}$ and $R_{2}$.

This product is the prototype of a protein, and the distance between two consecutive $\mathrm{C}_{\alpha}$ atoms is $3.8 \AA$. Natural proteins typically consist of hundreds of amino acids. It is the variety of the twenty amino acid's side chains which leads to the great diversity among proteins. As an example, figure 1.1 shows the three-dimensional structures of glycine (G), serine (S) and tryptophan (W), which are the most relevant amino acids for this thesis. The side chains of $\mathrm{G}$ and $\mathrm{S}$ are in fact the shortest among all amino acids. In a physiological environment, proteins are not straight lines, but often fold into a stable three-dimensional structure, both locally on basis of a few amino acids (secondary structure), and also the entire molecule as a whole (tertiary structure). Based on the tertiary structures, they can perform their specific biological functions, and often a sort of "lock-and-key" principle applies between the two. Over the course of about 4 billion years on earth, a self-organized trial-and-error algorithm called evolution has yielded a great number of amino acid sequences which make biologically useful proteins. Today, the famous protein folding problem is still unsolved; we cannot predict a protein's three-dimensional structure, let alone its biological function, based on a given amino acid sequence. In the future, such predictions may enable us to design proteins performing desired tasks in a living organism. In recent years, it has become known that many proteins contain parts which do not fold into stable secondary or tertiary structures at all, but keep on fluctuating ("random coil"), earning them the name "intrinsically disordered protein" (IDP). In fact, more than $30 \%$ of all eukaryotic proteins are IDPs; 
but even regularly folding proteins are initially disordered when they are chemically formed, and thus the dynamics of IDPs sets an upper speed limit for protein folding. Within the scope of this thesis, we can think of IDPs as random coils which constantly undergo conformational changes due to thermal fluctuations - or as the perfect playground for polymer physics.
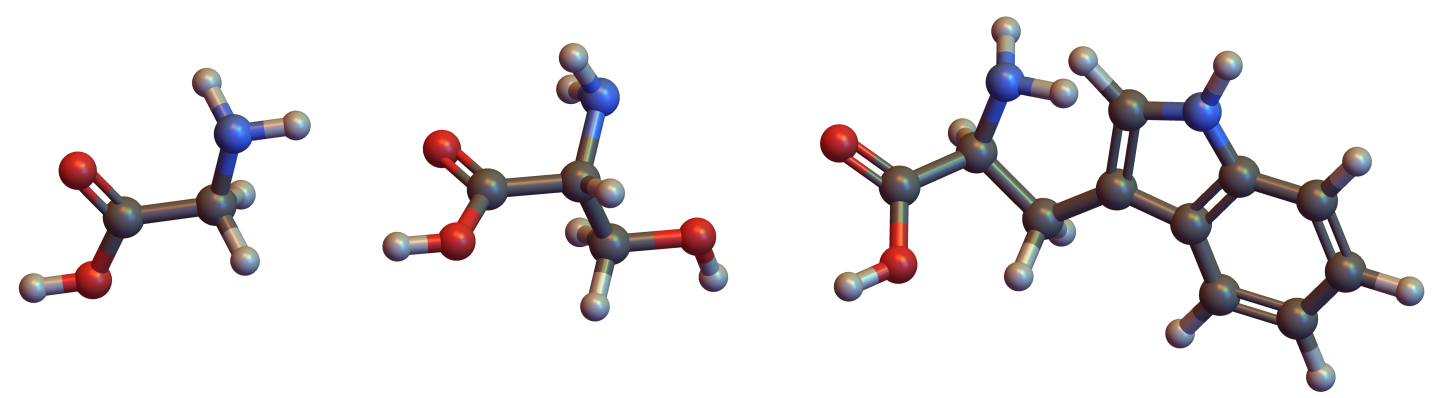

Figure 1.1: The three-dimensional structures of the three amino acids glycine (left), serine (center) and tryptophan (right). The beads represent carbon (gray), oxygen (red), hydrogen (white) and nitrogen (blue) atoms. The backbones can be found at the respective molecule's top left (C-C-N), and the side chains branch out from the central carbon atom towards the bottom right.

Situation at hand The past few decades have witnessed great progress in single-molecule measurements. It is now possible to obtain information not only about a molecule's statistical properties (e.g. its radius of gyration measured from small-angle x-ray scattering (SAXS) or its force-extension curve measured from atomic force spectroscopy (AFM)), but also to measure dynamical properties like the rate with which a linear polymer's two ends diffusively come together (called loop formation rates or contact rates). These are truly exciting times: we are in the process of creating a link between modern physics and biological processes by subjecting proteins to single-molecule experiments. The group I have worked with is pushing the frontier of what can be achieved on this scale, with my colleagues employing state-of-the-art singlemolecule techniques and continuing to develop new ones. At the time when I started my doctoral work in June 2016, Man Zhou had already measured loop formation rates and hydrodynamic radii of a number of IDPs called GS-repeats, using photo-induced electron transfer combined with fluorescence correlation spectroscopy (PET-FCS) and dual-focal fluorescence correlation spectroscopy (2fFCS) respectively. A GS-repeat is a short protein (10 to 40 amino acids long) whose amino acid sequence alternatingly consists of glycine $(G)$ and serine $(\mathrm{S})$. They are known to be flexible, intrinsically disordered proteins lacking secondary structures and showing no internal friction [3]. Their dynamics take place on the nanoscale, both temporally as well as spatially. We aim to translate the experimental findings into characteristic properties of these GS repeats, such as their persistence length, and interpret the results via a custom variant of polymer theory. 
Problem statement We require a polymer model which enables us to interpret data from our single-molecule experiments for short proteins. In particular, the model must be able to predict values for contact rates as well as hydrodynamic radius values. It is not required that the model is analytically solvable by any means; computational treatability suffices. The model should be reasonably realistic while keeping the total number of free parameters to a minimum not only (i) to avoid overfitting, but also because (ii) every additional dimension in parameter space makes finding the best-fitting parameter set exponentially more expensive from a numerical perspective. On the other hand, we want to be in control of the model's behavior and test which mechanisms are important to match the experimental data, and this requires at least one tunable model parameter. The experimentally determined time between loop formation events in proteins (inverse contact rate) is on the order of tens or hundreds of nanoseconds, and thus simulated trajectory lengths should be at least on the order of microseconds to estimate the simulated contact rate with a reasonable level of convergence. Given such a model, we would like to develop a combined routine of performing experiments with different molecules (i.e. different amino-acid sequences in proteins) and assigning properties (e.g. their persistence length) to these molecules by fitting the experimental results using our model.

State of the art Several experimental studies quantified the rate at which two sites on a peptide chain come together (contact rate $k_{+}$) [3-13] and found a power law dependence of the loop formation rate in disordered proteins on chain length $[3,6,7]$ as long as the chains are no shorter than about 10 amino acids [14].

Theoretical studies delivered explicit (yet approximate) expressions for contact rates which can be evaluated once a polymer model has been chosen. The most commonly used polymer models are Gaussian chains [15-17] which, inspired by the central limit theorem, are characterized by having Gaussian statistics between all of their monomers. A comprehensive treatment (including hydrodynamic interactions and internal friction) of Gaussian chains can be found in reference [18]. The Gaussian property leads to linear equations of motion, but it turns out that even for the simplest Gaussian polymer models, simplifying assumptions must be made to calculate contact rates analytically. The Szabo-Schulten-Schulten (SSS) theory [19] considers the end-to-end dynamics of the polymer to be Markovian, which leads to an explicit expression for the contact rate. Kramers theory [20] also delivers (approximate) scaling properties of the contact rate from a one-dimensional picture. The Wilemski-Fixman (WF) [21, 22] theory takes into account that the polymer dynamics are high-dimensional, but this comes at the cost of making the so-called closure approximation necessary to obtain an expression for the contact rate. The FriedmanO'Shaughnessy theory [23-25] is more concerned with the scaling behavior of the contact rate rather than delivering realistic values. These theories have been widely used for simple polymer models [26-35], yielding important understandings of the dynamics of loop formation. 
Power laws for the contact rate as a function of chain length are often found, and it turns out that different scaling regimes (depending on the critical contact radius for loop formation, chain length and persistence length) with different power law exponents exist as long as the chains are significantly longer than their persistence length. However, all currently available theories require numerical evaluation for non-oversimplified polymer models, and even then only yield approximate contact rate values within those models. Since we do not seek analytical results but want to fit experimental data quantitatively, we conclude that no currently available theory exists which serves our purpose. Instead, it is advantageous for us to formulate a fairly realistic polymer model, and extract the contact rate from it using simulations rather than analytics.

Computational studies have also found the predicted power-law-like scaling behavior [36-39] and deviations from it for short, semiflexible chains (i.e. chains with finite bending stiffness) [40, 41]. Computational models of fluctuating polymers can be separated into two categories: Those using an explicit fluid, and those using an implicit fluid. As the name suggests, explicit-fluid models represent the heat bath surrounding the polymer of interest via explicit variables whose dynamics are simulated along with the polymer's coordinates. Simulating each water molecule explicitly is known as a molecular dynamics (MD) simulation. Modern MD simulations for proteins such as one of our GS-repeats need to simulate around 20000 water molecules explicitly and reach around $100 \mathrm{~ns}$ per day, if run on 64 processors [42-44]. Since sufficiently long trajectories, from which contact rates on the order of $1 / 100 \mathrm{~ns}$ can be estimated, therefore take months of computation time for each tested chain length, MD is eliminated as a potential quick and efficient tool to interpret our single-molecule experiments. Implicit-fluid techniques, on the other hand, only simulate the polymer's coordinates and combine their dynamics with hydrodynamic theory to capture the fluid's impact on the chain. This impact is three-fold: friction (energy dissipation), hydrodynamic interaction (coupled movement of objects in the fluid) and thermal fluctuations (stochastic momentum transfer). These three effects need to be treated approximately for implicitfluid models, while they emerge automatically in explicit-fluid models (which also means they cannot be turned on or off). Implicit-fluid models represent the molecule with a chosen spatial resolution - one bead in the model represents one amino acid for instance, or even a large number of them, depending on the situation at hand. Hydrodynamic interactions are nonlinear, pairwise interactions of the beads, severely limiting the number of beads which can be handled numerically. For this reason, many polymer models neglect hydrodynamic interactions, and in pursuit of capturing them in an emergent manner - explicit fluid techniques representing the solvent more coarsely than MD does exist. Such "mesoscale hydrodynamics" or "coarsegrained fluid" techniques (including lattice-Boltzmann [45], dissipative-particle dynamics [46] and multiparticle-collision dynamics [47]) are well-suited for simulating hundreds of beads, but are being outperformed by implicit-fluid models (with hydrodynamic interactions) in both accuracy and speed [48] the smaller the system of interest is (short chains). 
We are therefore in the sweet spot where numerical simulations with an implicit fluid are both required and possible: Our proteins are too long for MD, too short for Gaussian analytics, and short enough to include hydrodynamic interactions in an implicit fluid.

Model structure The polymer model developed and applied in this thesis is a semiflexible beadrod chain with hydrodynamic interactions and excluded volume effects. Each bead represents one amino acid, and the fluorophore is represented by one additional larger bead at one chain end. The distances between neighboring beads (bond lengths) are constant over time, and thus the model underlies geometric constraints. The model has two free parameters. The first one is the chain's bending stiffness $\sigma$ which enters the model from within the framework of discrete differential geometry as a prefactor of its bending energy. It quantifies how much energy is required to bend the chain and can be bijectively mapped onto the chain's persistence length. $\sigma$ entirely determines the chain's statistical properties via the Boltzmann distribution. The second free parameter is the hydrodynamic bead radius $a$ of a single bead (amino acid) in the chain. It enters the model from within the framework of hydrodynamics via the Rotne-Prager diffusion matrices. Given $\sigma$, it entirely determines the model's dynamical properties.

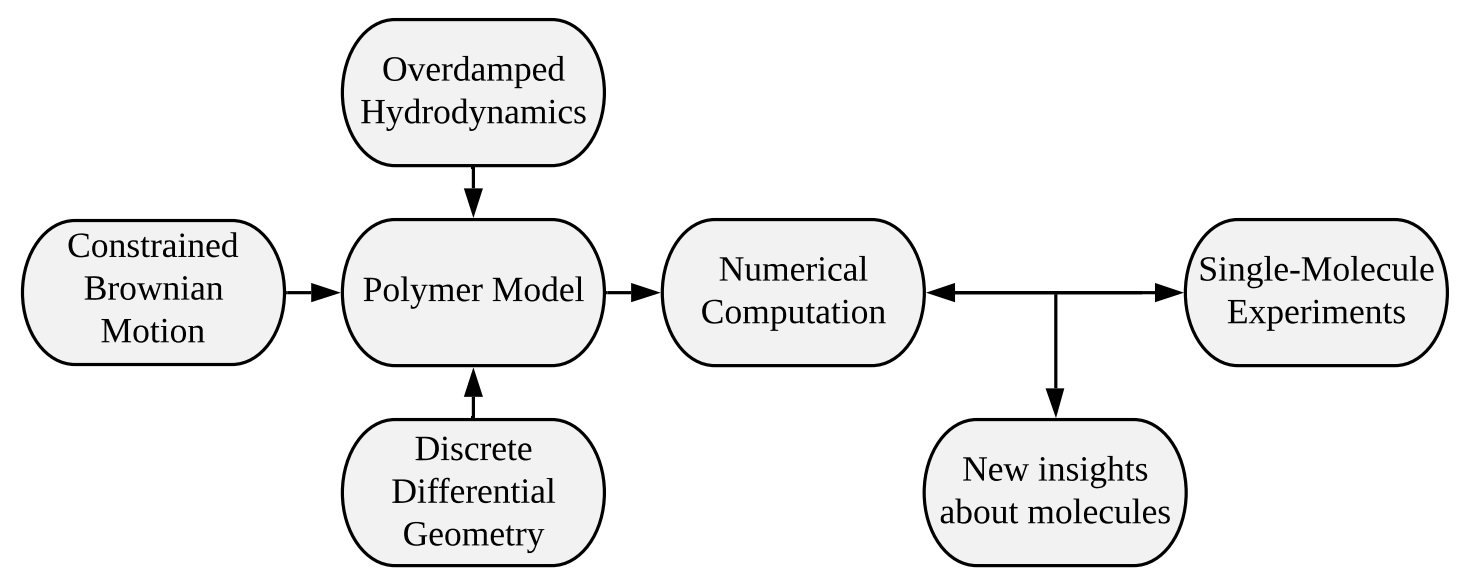

Figure 1.2: We combine the theories of hydrodynamics, Brownian motion and differential geometry into a polymer model. Results from single-molecule experiments can be compared with those predicted by the model, which yields quantitative information about the investigated molecules. 
Philosophy behind modeling A mathematical model of a complex system yields an imaginative, simplified reality. Unlike in the real world, the rules governing this reality are perfectly known to us and are ours to modify as we please. We can control this alternative reality from the outside by erasing rules, introducing new ones or by changing the values of model parameters. Meanwhile, the model predicts values for observables (such as the contact rate of a polymer) which can be compared with values measured in the real world. Since the importance and fun of understanding which rules lead to which behavior often seem incomprehensible to our peers, it is fortunate that building a model also has pragmatic justifications:

- We can tune parameter values such that the model's prediction matches the measured reality as well as possible. Properties of the real-world system can then be deduced from these parameter values. For instance, we can tune our polymer model's persistence length such that the measured contact rates are reproduced, and then attribute this persistence length to the studied proteins.

- We can cleanly test how altering the parameter values changes the observables. In reality, it is often impossible to e.g. change the protein's persistence length without also changing the solvent's viscosity or the amount of ions in it.

- Modeling allows us to turn physical effects on or off which are irrefutable in reality. For instance, it is easy for us to allow the polymer chain to cross through itself or turn off hydrodynamic interactions, while in reality this is clearly impossible. Consequently, it can e.g. be tested if the fact that the proteins cannot cross through themselves is relevant for their dynamics.

- Having at hand complete information about the model's output, it is possible to extract information which is in reality expensive, time-consuming, unethical or even impossible to measure.

I would like to emphasize that our mathematical polymer model is derived from physical principles. Rather than heuristically writing down a mathematical model which is taylored to predict specific observables, we are following a "bottom-up" approach and only after having the model at hand do we specify which observables (contact rate, hydrodynamic radius, reconfiguration time) we extract from it. In particular, we are not designing the model such that it automatically delivers some desired or experimentally observed behavior, as this would be pointless but alas not unheard of. 
Thesis structure A list of symbols and acronyms, as well as the conventions for our mathematical notation can be found right before this chapter.

In chapter 2, the existing theories of hydrodynamics, statistical mechanics, differential geometry and polymer theory are introduced in this order. The section on hydrodynamics first derives the continuity equation and the Navier-Stokes equation, introduces the concept of the Reynolds number and then finds the (overdamped) Stokes-equation. Next, the dynamics and hydrodynamic interactions of a number of spheres embedded in a Stokes-flow are investigated within the Rotne-Prager approximation, resulting in an analytical expression for the diffusion matrix. The section on statistical mechanics starts with the basic thermodynamic principles governing systems in thermal equilibrium (free energy minimization, the Boltzmann distribution etc.) and basic concepts of stochastic processes, in particular of Markov processes. From there, we derive the Fokker-Planck equation as the Kramers-Moyal expansion of the Master equation. We show the equivalence of the Fokker-Planck equation with the Langevin equation the stochastic differential equation governing our polymer model's dynamics - and demonstrate how to impose geometric constraints on the dynamics using Lagrangian multipliers. The section on statistical mechanics ends with introducing the diffusion coefficient, hydrodynamic radius and the Stokes-Einstein relation. The section on differential geometry is concerned with onedimensional, framed curves in three-dimensional space. First the case of a continuous Kirchhoff rod is treated in a Lagrangian manner. Afterwards, we study its discrete analogue, arriving at analytical expressions for the elastic forces which are present in our polymer model. Next we give a brief overview of aspects of polymer theory which are important for us. By the end of chapter 2, we introduce the experimental methods of single-molecule spectroscopy (SMS), fluorescence correlation spectroscopy (FCS), photo-induced electron transfer (PET), dynamic metal-induced energy transfer (dynaMIET) and dual-focus fluorescence correlation spectroscopy (2fFCS).

In chapter 3, we assemble the ingredients from chapter 2 and compose our polymer model.

In chapter 4, we explain the computational methods utilized to breath life into the model. To this end, we first nondimensionalize the model equations and then explain the numerical integration scheme used for the Langevin equation in the presence of geometric constraints. Next, we introduce a method called Monte-Carlo sampling (MC) which allows the evaluation of ensemble averages without running a simulation, and demonstrate how to use it for calculating the hydrodynamic radius of a polymer chain undergoing conformational dynamics. After showing how to calculate the contact rate from a simulated trajectory, we conclude chapter 3 by benchmarking our model and numerical methods by checking that they reproduce known analytical results. 
In chapter 5, the main results of this thesis are presented. We start off by investigating how the model parameters influence its behavior without considering experimental results yet. Next comes what I consider the main project of this thesis: Loop formation and translational diffusion of GS-repeats investigated using PET-FCS and 2fFCS. Here we find that the model excellently reproduces experimental results, and are able to assign a persistence length of $5.2 \pm 1.9 \AA$ to the measured proteins, as well as a hydrodynamic radius of $3.5 \pm 0.7 \AA$ to their individual amino acids. In the next, very similar, project, it turns out that the model fails in reproducing the experimental results for a different type of protein (FG-proteins) which has a highly heterogeneous amino acid sequence. Lastly, we apply the model to a different experimental setup: the reconfiguration dynamics of DNA molecules being bound to a surface investigated via dynaMIET. The model predicts the DNA's dynamics to take place on a time scale of tens to hundreds of nanoseconds.

In chapter 6, we discuss our findings and conclude the thesis.

In chapter 7, we suggest possible directions of future work which may succeed this thesis, and show preliminary results in some of these directions.

In chapter 8, four side-projects that I have been working on during my doctoral dissertation are briefly presented. These are (i) multiplane imaging of flagellar motion, (ii) geometric constraints in protein folding, (iii) electric field lines of arbitrarily moving point charges and (iv) kinetics of loop closure in disordered proteins.

The appendices include details or calculations which were removed from the thesis for readability, and are referred to in the main text.

How to read this thesis? I recommend one of two options:

1. You can read this thesis from front to back, learning about all theoretical concepts step by step before they are assembled into our polymer model.

2. You can jump straight to the model definition (chapter 3) and treat chapter 2 as a source of reference to read up on individual concepts as needed. The numerical methods (chapter 4), as well as the outlook (chapter 7) and side projects (chapter 8) may be skipped as well. 
Historical context Fluid dynamics is described by the well-known Navier-Stokes equation which was discovered in the 1820s [49] by Claude-Louis Navier. In the decades after, significant distributions were made by Sir George Gabriel Stokes [50], including the introduction of the Reynolds number. This lead to the overdamped Navier-Stokes equation (called the Stokes equation), the solution of which we require in this thesis to quantify hydrodynamic interactions and solvent friction. Its Green's function is called the Oseen matrix which was published in 1910 [51]. Taking into account that the objects in the fluid have a finite size is achieved by using the so-called Rotne-Prager diffusion matrix. It was originally calculated by Rotne and Prager [52] and Yamakawa [53] in the 1960s and 1970s. We require an analytical expression of this matrix for spherical beads of finite size which are potentially different-sized and overlapping. Remarkably, this expression was only found in 2015 [54, 55].

Building on the 19th century's work on statistical mechanics and thermodynamics performed by Maxwell, Boltzmann, Clausius, Gibbs and others [56], the theory of Brownian motion was mainly developed in the early 20th century by Smoluchovski [57], Langevin [58], Fokker [59], Planck [60] and Einstein [61]. The combination of Brownian dynamics with overdamped hydrodynamics, which we use in this thesis, goes back to Ermak and McCammon's work in 1978 [62]. Within the framework of Brownian motion, our model is subject to geometric constraints. Constrained Brownian motion is an ongoing research topic [63] (2017), first treated by Kramers [64] (1946) and Fixman [65] (1974). In particular, the evaluation of the entropic pseudoforce we use later on was described in 2002 by Pasquali and Morse [66], and the numerical integration algorithm in 2005 by Montesi et al [67].

Meanwhile, polymer theory can be regarded as a branch of statistical mechanics which has seen major developments since the middle of the 20 th century. The worm-like chain (an elastic rod in a heat bath) is the standard model for a polymer with finite bending stiffness, and was first published by Kratky and Porod [68] in 1949. Flory's work on chains with excluded volume effects was published in 1953 [69]. The most paradigmatic polymer model whose dynamics can be treated analytically because it is Gaussian is the Rouse chain [15] (1953), which was later on generalized to include hydrodynamic interaction (Zimm chain [16], 1956) and internal friction (RIF chain [70], 2007). With the work on stochastic processes by Zwanzig, van Kampen, Risken, Mazo and others in the 1970 s, and with the recent advent of computational power, the dynamics of nonlinear polymer models which are only numerically accessible has garnered more and more attention since the beginning of this century. In the last 10 years in particular, beads models have been used to study intrinsically disordered proteins [71-73]. 
The theory of elastic rods with an energy function which is quadratic in its curvature is an ancient paradigm, already studied by Leonhard Euler and Daniel Bernoulli in the 18th century [74]. Major progress is due to Kirchhoff's work [75] in the 19th century, who described elastic rods as framed curves using an orthonormal triad. The efficient description of the triad's evolution along the curve goes back to Bishop's work in 1975 [76]. Using local rather than Cartesian coordinates, a Lagrangian treatment of such framed curves was described in 1996 by Langer and Singer [77], and physical dynamics of inextensible rods within this framework were described shortly after (1998) by Goldstein et al [78]. In this thesis, we use the more recently developed theory of discrete differential geometry (2010 by Bergou et al. [79]) to describe discrete chains in a Lagrangian manner and calculate the elastic forces acting on them. Discretizing an intrinsically continuous theory for numerical purposes is thereby avoided, and instead the theory itself treats the chain as a discrete object.

The first direct, optical detection of a single molecule in a liquid was achieved by Hirschfeld [80, 81] in 1976. In 1996, Keller et al. [82, 83] detected individual molecules in a fluid flow. The field of single-molecule spectrocopy (SMS) has since seen vast developments. In particular, it was combined with fluorescence correlation spectroscopy (FCS) which had already been developed in the 1970s [84-86], but not yet been applied to single-molecule data. Experimental techniques which, when combined with SMS and FCS, allow the investigation of a single molecule's dynamics are Förster-resonance energy transfer (FRET), triplet-triplet energy transfer (TTET) and photoinduced electron transfer (PET), all of which have been available since around the year 2000 . TTET and PET, in particular, allow measuring loop formation rates in single molecules, with PET being the more "direct" method because, in contrast to TTET, quenching of the excited fluorophore occurs virtually instantaneously upon loop closure. PET-FCS has been used since the beginning of this century to study loop formation rates in IDPs. The hydrodynamic radius of single molecules (e.g IDPs) can be measured using dual-focal fluorescence correlation spectroscopy (2fFCS), which was developed in 2007 by Dertinger et al [87].

To the best of my knowledge, we are the first to systematically use both 2fFCS and PET-FCS to simultaneously measure translational diffusion and loop formation of short IDPs of varying chain lengths, and develop an efficient, quantitative fitting routine to interpret the data. 


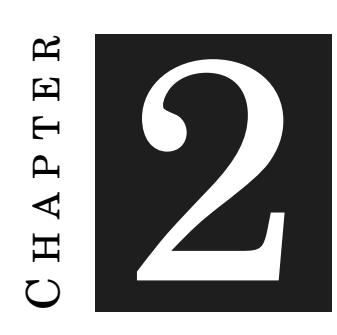

\section{THEORY}

T $\mathrm{n}$ this chapter the physical and mathematical concepts which are being utilized in this thesis Lare introduced. Roughly starting at the level of a physics graduate, its contents can either be found in advanced text books, which we name at the beginning of the respective section, or in published manuscripts we cite at the appropriate places. I would thus like to make clear that none of the results in this chapter were originally derived by me. Our aim here is not to replace or summarize full textbooks on the treated subjects.

Instead, an effort will be made to streamline them towards their aspects which are relevant for our polymer model, thereby combining hydrodynamics, statistical mechanics and differential geometry into a global picture which can be understood from the perspective of polymer theory.

\subsection{Hydrodynamics}

When a force acts on a classical body in a vacuum, Newton's second law allows us to calculate its velocity. In this thesis, however, we are not considering an object in a vacuum, but microscopic objects surrounded by a fluid - a continuum of water. It is dense enough for microscopic volumes $\mathrm{d} V$ to still be treatable as a continuum of water. Instead of talking about individual molecules, the fluid's state is described by its mass density $\rho$, velocity $\vec{v}$ and pressure $p$ as functions of both space $\vec{r}$ and time $t$. The objects embedded in this fluid are the vertices of a discrete polymer chain which are treated as spherical beads. The entire purpose of this section is to calculate the beads' velocities when the forces acting on them are known. This is achieved as follows: We derive the continuity equation, the Navier-Stokes equation, and their incompressible, overdamped version: the Stokes equation. Next, spherical beads will be embedded in the Stokes flow, where 
hydrodynamic interactions and solvent friction are quantified by the diffusion matrix $\mathbf{D}$. The derivation of this matrix is ultimate goal of this section.

Unless explicitly stated otherwise, we follow the line of reasoning from the textbook "An Introduction to Dynamics of Colloids" by Jan Dhont [88].

\subsubsection{Continuity equation}

The continuity equation describes the conservation of mass in a fluid. Considering any volume $V$ fixed in space, it balances the influx and outflux of fluid into that volume as follows. The change of the total mass $M$ in $V$ changes only due to fluid flux $\vec{j}=\rho \vec{v}$ through its boundary $\partial V$ :

$$
\begin{aligned}
\mathrm{d}_{t} M & =\mathrm{d}_{t} \int_{V} \rho \mathrm{d} \vec{r}=\int_{V} \partial_{t} \rho \mathrm{d} \vec{r} \\
& \stackrel{!}{=}-\int_{\partial V}(\vec{j} \cdot \vec{n}) \mathrm{d} A=-\int_{V}(\vec{\nabla} \cdot \vec{j}) \mathrm{d} \vec{r}=-\int_{V} \vec{\nabla} \cdot(\rho \vec{v}) \mathrm{d} \vec{r}
\end{aligned}
$$

where $\vec{n}$ is the normal vector of $\partial V$ (pointing outwards, thus the minus sign). Gauss' integral theorem was used in the last line and Reynold's transport theorem in the first line. The transport theorem simply states that for our case of a fixed volume $V$, the total time derivative may be pulled into the integral, becoming a partial derivative. Since $V$ is arbitrary, the integrands of both integrals must coincide and thus we have

$$
\begin{aligned}
0 & =\partial_{t} \rho+\vec{\nabla} \cdot(\rho \vec{v}) \\
& =\mathrm{d}_{t} \rho+\rho \vec{\nabla} \cdot \vec{v}
\end{aligned}
$$

This equation is a partial differential equation coupling the evolution of $\rho$ and $\vec{v}$. In this thesis, we are dealing with incompressible fluids such that $\rho$ is a constant. The continuity equation therefore reduces to the incompressibility equation, which constrains the fluid flow to obey

$$
\vec{\nabla} \cdot \vec{v}=0
$$

\subsubsection{Navier-Stokes equation}

Newton's second law states that the momentum $\vec{P}$ of an object changes according to the total force $\vec{F}=\mathrm{d}_{t} \vec{P}$ applied to it. We take this object to be an arbitrary volume $V$ which is fixed in space as before. Its momentum balance reads

$$
\begin{aligned}
\mathrm{d}_{t} \vec{P} & =\mathrm{d}_{t} \int_{V} \rho \vec{v} \mathrm{~d} \vec{r}=\int_{V} \partial_{t}(\rho \vec{v}) \mathrm{d} \vec{r} \\
& \stackrel{!}{=} \int_{\partial V} \rho \vec{v} \cdot(\vec{v} \cdot \vec{n}) \mathrm{d} A+\int_{V} \vec{f} \mathrm{~d} \vec{r}+\int_{\partial V}(\hat{\boldsymbol{\sigma}} \cdot \vec{n}) \mathrm{d} A
\end{aligned}
$$

The first line is a mathematical identity, and the second line consists of three terms which contribute to a change of momentum in $V$. Firstly, there is a convective flow of momentum density $\rho \vec{v}$ through the boundary of $V$. Secondly, in general, an external force density $\vec{f}$ acts within $V$. 
Thirdly, a short ranged force $\hat{\boldsymbol{\sigma}} \cdot \vec{n}$ acts on the surface of $V$. This force is conveniently expressed as the matrix product of the stress tensor $\hat{\boldsymbol{\sigma}}$ with the surface's normal vector $\vec{n}$ such that Gauss' integral theorem can be applied. The stress tensor's entries for incompressible Newtonian fluids such as water read (see page 233 in ref. [88])

$$
\hat{\sigma}_{i j}=-p \delta_{i j}+\eta\left(\partial_{j} v_{i}+\partial_{i} v_{j}\right)
$$

where the first term describes the fluid's pressure $p$ (a force density per area) experienced by $V$ on its surface. The second term describes the friction $V$ experiences from interacting with surrounding fluid cells which may have different velocities. This friction is proportional to the local velocity gradient and the fluid's viscosity $\eta$ acts as the proportionality factor. The property that this friction force is linear in the velocity gradient defines both Newtonian fluids and $\eta$.

Making use of the incompressibility equation as well as Gauss' integral theorem for every component of $\rho \vec{v}$ and every row of $\hat{\boldsymbol{\sigma}}$, we again equalize the integrands in equation 2.4 because $V$ is arbitrary. This leads to a partial differential equation which the fluid's flow must obey:

$$
\rho \cdot\left(\partial_{t} \vec{v}+(\vec{v} \cdot \vec{\nabla}) \vec{v}\right)=\eta \vec{\nabla}^{2} \vec{v}-\vec{\nabla} p+\vec{f}
$$

It is called the Navier-Stokes equation for incompressible Newtonian fluids. Save for the diffusive term $\vec{\nabla}^{2} \vec{v}$, which is a material property, it is as exact as Newton's second law together with the incompressibility condition. We only need to keep in mind that we are treating the fluid as a continuum of mass rather than individual molecules.

\subsubsection{Stokes equation}

In many applications, approximate knowledge of the orders of magnitude that the variables in the Navier-Stokes equation take on is available. For example, when a person is swimming in water, a typical length scale is that person's height, and a typical velocity might be $2 \mathrm{~km} / \mathrm{h}$. Let us assume that for some abstract application, a length scale $r_{0}$, a velocity scale $v_{0}$, as well as the fluid's mass density $\rho$ and viscosity $\eta$ are given. One can then make use of these scales and nondimensionalize the Navier-Stokes equation by introducing the primed variables $\vec{r}=r_{0} \vec{r}^{\prime}$, $\vec{v}=v_{0} \vec{v}^{\prime}$ and so forth. The dashed variables are thus dimensionless by construction and can be expected to take on values which are approximately of order 1 . Choosing a time scale $t_{0}=r_{0} / v_{0}$, a pressure scale $p_{0}=v_{0} \eta / r_{0}$ and a force scale $f_{0}=\eta v_{0} / r_{0}^{2}$, and inserting the primed quantities into the Navier-Stokes equation yields

$$
\begin{aligned}
\left(\rho v_{0}^{2} / r_{0}\right) \cdot\left(\partial_{t^{\prime}} \vec{v}^{\prime}+\left(\vec{v}^{\prime} \cdot \vec{\nabla}^{\prime}\right) \vec{v}^{\prime}\right) & =\left(\eta v_{0} / r_{0}^{2}\right) \cdot\left(\vec{\nabla}^{\prime 2} \vec{v}^{\prime}-\vec{\nabla}^{\prime} p^{\prime}+\vec{f}^{\prime}\right) \\
\Leftrightarrow \operatorname{Re} \cdot\left(\partial_{t^{\prime}} \vec{v}^{\prime}+\left(\vec{v}^{\prime} \cdot \vec{\nabla}^{\prime}\right) \vec{v}^{\prime}\right) & =\vec{\nabla}^{\prime 2} \vec{v}^{\prime}-\vec{\nabla}^{\prime} p^{\prime}+\vec{f}^{\prime}
\end{aligned}
$$

where we have introduced the Reynolds number

$$
\operatorname{Re}=\frac{\rho r_{0} v_{0}}{\eta} .
$$


Since all appearing terms are of order 1, the Reynolds number dictates how relevant the inertial terms (left hand side) are compared to the acting forces (right hand side). If it is very large, inertial effects dominate the fluid's dynamics, which leads to turbulence (starting at around $\operatorname{Re} \approx 2000$ ). Throughout this thesis, we assume that Re is small enough to neglect inertial terms and therefore set the left hand side of equation 2.7 to zero. Given the values of $\rho$ and $\eta$, this is appropriate for small velocity and length scales; it is thus a much better approximation for microscopic objects than it is for whales. In our applications later on, estimating the Reynolds number yields values on the order of $10^{-2}$, which makes the neglect of inertial effects reasonable. The corresponding Navier-Stokes equation is called the Stokes equation and reads

$$
0=\vec{\nabla}^{2} \vec{v}-\vec{\nabla} p+\vec{f}
$$

It is linear and time does not enter it, and therefore the fluid's velocity $\vec{v}$ and pressure $p$ must be linear functions of the applied force density $\vec{f}$ at each individual instant of time. As a direct consequence, the fluid is immediately at rest when forces are no longer applied to it. This has astonishing physical implications on microscopic swimming mechanisms, which are beautifully demonstrated in Purcell's famous paper "Life at low Reynolds number" [89]. Due to these properties, the Stokes equation and the incompressibility equation taken together are also called the creeping flow equations. From a mathematical perspective, the four creeping flow equations form a set of equations for the four variables $\vec{v}(\vec{r})$ and $p(\vec{r})$. We do not consider the force density $\vec{f}$ as a variable, but as given input at each time, for which the equations are to be solved independently of all other times because of the lack of inertial effects. Due to the linearity of the creeping flow equations, the dependence of $\vec{v}$ and $p$ on $\vec{f}$ must be linear superpositions of the form

$$
\begin{aligned}
& \vec{v}(\vec{r})=\int\left(\boldsymbol{T}\left(\vec{r}-\vec{r}^{\prime}\right) \cdot \vec{f}\left(\vec{r}^{\prime}\right)\right) \mathrm{d} \vec{r}^{\prime} \\
& p(\vec{r})=\int\left(\vec{g}\left(\vec{r}-\vec{r}^{\prime}\right) \cdot \vec{f}\left(\vec{r}^{\prime}\right)\right) \mathrm{d} \vec{r}^{\prime}
\end{aligned}
$$

where the matrix $\boldsymbol{T}$ and the vector $\vec{g}$ are Green's functions for the creeping flow equations. They can be found explicitly by inserting equation 2.10 into equations 2.3 and 2.9. Using the boundary condition that the velocity field drops to zero at $|\vec{r}| \rightarrow \infty$, one finds that

$$
\boldsymbol{T}(\vec{r})=\frac{1}{8 \pi \eta} \frac{1}{|\vec{r}|}\left(\rrbracket_{3 \times 3}+\frac{\vec{r} \cdot \vec{r}^{T}}{|\vec{r}|^{2}}\right)
$$

$$
\text { and } \quad \vec{g}(\vec{r})=\frac{1}{4 \pi} \frac{\vec{r}}{|\vec{r}|^{3}} \quad .
$$

An identity we require later on is

$$
\int_{\partial S} \boldsymbol{T}\left(\vec{r}-\vec{r}^{\prime}\right) \mathrm{d} A^{\prime}=\frac{2 a}{3 \eta} \mathbb{1}_{3 \times 3} \quad \text { where } \quad \vec{r} \in \partial S
$$

and $S$ is a sphere with radius $a$. The matrix $\mathbf{T}$ is called the Oseen-matrix [51,90] and satisfies $\mathbf{T}(\vec{r})=\mathbf{T}(-\vec{r})$. Its explicit derivation from the creeping flow equations is straightforward but lengthy, and we refer the interested reader to the mentioned textbooks or to reference [91]. 


\subsubsection{Spheres in a Stokes flow}

Let us consider a single sphere $S_{1}$ with radius $a$ embedded in a Stokes flow with a total nonhydrodynamic force $\vec{F}_{1}$ and no torques acting on it. The sphere resides at position $\vec{r}_{1}$ with translational velocity $\vec{v}_{1}$ and angular velocity $\vec{\omega}_{1}$. The force $\vec{F}_{1}$ is equally distributed over the surface $\partial S_{1}$ of $S_{1}$. Thus, the force density determining the fluid's velocity via equation 2.10 is

$$
\vec{f}_{1}=\frac{1}{4 \pi a^{2}} \delta\left(\left|\vec{r}-\vec{r}_{1}\right|-a\right) \vec{F}_{1}
$$

Furthermore, the presence of the sphere imposes no-slip boundary conditions on the fluid flow, which read

$$
\vec{v}_{1}+\vec{\omega}_{1} \times\left(\vec{r}-\vec{r}_{1}\right)=\vec{v}(\vec{r})=\frac{1}{4 \pi a^{2}} \int_{\partial S_{1}^{\prime}}\left(\boldsymbol{T}\left(\vec{r}-\vec{r}^{\prime}\right) \cdot \vec{F}_{1}\right) \mathrm{d} A^{\prime} \quad \text { where } \vec{r} \in \partial S_{1}
$$

which simply means that there is no relative velocity between fluid and surface elements on $\partial S_{1}$. While it is clear why the fluid cannot possess a velocity component through the sphere's surface, the physical reason why there is no component parallel to it is the following [92]. At the microscopic level, there are interaction (adhesion) forces between surface and water molecules which statistically reduce their relative velocity upon collision. As a result of the short mean free path of water molecules, i.e. the distance they travel before colliding with another water molecule, each water molecule near the surface collides with it many times. Therefore, the average fluid velocity in any small volume $V$ at the surface loses its velocity component parallel to the wall, even though individual water molecules within $V$ may not be at rest. The no-slip boundary condition can thus be understood as a consequence of the continuum description of a dense fluid which microscopically interacts with the surface. It fails when the fluid is too diluted, or when its molecules only elastically bounce off a (hydrophobic) surface.

Stokes law Integrating the boundary conditions over $\partial S_{1}$ eliminates the rotational term on the left hand side and, using equation 2.12 , leads to

$$
\begin{aligned}
4 \pi a^{2} \vec{v}_{1} & =\frac{1}{4 \pi a^{2}} \int_{\partial S_{1}} \int_{\partial S_{1}^{\prime}}\left(\boldsymbol{T}\left(\vec{r}-\vec{r}^{\prime}\right) \cdot \vec{F}_{1}\right) \mathrm{d} A^{\prime} \mathrm{d} A \\
& =\frac{1}{4 \pi a^{2}} \int_{\partial S_{1}} \frac{2 a}{3 \eta} \vec{F}_{1} \mathrm{~d} A \\
& =\frac{2 a}{3 \eta} \vec{F}_{1} \\
\Leftrightarrow \gamma \vec{v}_{1} & =\vec{F}_{1},
\end{aligned}
$$

which is the famous Stokes law for a sphere with radius $a$ in an overdamped fluid: The linear relation between its velocity and the force acting on it is quantified by the friction coefficient

$$
\gamma=6 \pi \eta a \quad \text {. }
$$

Remarkably, this coefficient itself is a linear function of the sphere's radius $a$ (rather than its cross sectional area). 
Flow field around the sphere The velocity field $\vec{v}(\vec{r})$ caused by the sphere is given by inserting the force density 2.13 into equation 2.10 . The resulting integral can be solved analytically and, defining $\Delta \vec{r}=\vec{r}-\vec{r}_{1}$ and $\Delta r=|\Delta \vec{r}|$, reads

$$
\begin{aligned}
\vec{v}(\vec{r}) & =\frac{1}{4 \pi a^{2}} \int_{\partial S_{1}}\left(\boldsymbol{T}\left(\vec{r}-\vec{r}^{\prime}\right) \cdot \vec{F}_{1}\right) \mathrm{d} A^{\prime} \\
& = \begin{cases}\left(\frac{3}{4} \frac{a}{\Delta r}\left[1_{3 \times 3}+\frac{\Delta \vec{r} \cdot \Delta \vec{r}^{T}}{\Delta r^{2}}\right]+\frac{1}{4}\left(\frac{a}{\Delta r}\right)^{3}\left[1_{3 \times 3}-3 \frac{\Delta \vec{r} \cdot \Delta \vec{r}^{T}}{\Delta r^{2}}\right]\right) \cdot \vec{v}_{1} & \text { for } \quad R>a \\
\vec{v}_{1} & \text { for } R \leq a\end{cases}
\end{aligned}
$$

Note that this flow is continuous and satisfies the no-slip boundary conditions. It is visualized in figure 2.1. One may think of the inside of the sphere as consisting of fluid which is moving with the same velocity as the sphere's center and shell.

Dynamics of the sphere's center position Considering the translational dynamics of the sphere's center $\vec{r}_{1}$ instead of the fluid, the Stokes law 2.15 replaces Newton's second law (a second order, ordinary differential equation)

$$
m \mathrm{~d}_{t}^{2} \vec{r}=\vec{F}
$$

with the first order, ordinary differential equation

$$
0=-\gamma \mathrm{d}_{t} \vec{r}+\vec{F}
$$

Hence the widely used linear friction force $-\gamma \vec{v}$ is not a heuristic term, but is as exact as the Stokes equation.

Faxén's law So far we considered a sphere in a fluid which would be at rest without the force acting on that sphere. Now we are going to consider the same sphere embedded in a given velocity field $\vec{v}_{0}(\vec{r})$ (which satisfies the creeping flow equations) without specifying what this velocity field is caused by. Due to the linearity of the Stokes equation, we now have

$$
\vec{v}(\vec{r})=\vec{v}_{0}(\vec{r})+\frac{1}{4 \pi a^{2}} \int_{\partial S_{1}^{\prime}}\left(\boldsymbol{T}\left(\vec{r}-\vec{r}^{\prime}\right) \cdot \vec{F}_{1}\right) \mathrm{d} A^{\prime}
$$

This leads to modified no-slip boundary conditions at the sphere's surface, reading

$$
\vec{v}_{1}+\vec{\omega}_{1} \times\left(\vec{r}-\vec{r}_{1}\right)=\vec{v}(\vec{r})=\vec{v}_{0}(\vec{r})+\frac{1}{4 \pi a^{2}} \int_{\partial S_{1}^{\prime}}\left(\boldsymbol{T}\left(\vec{r}-\vec{r}^{\prime}\right) \cdot \vec{F}_{1}\right) \mathrm{d} A^{\prime} \quad \text { where } \vec{r} \in \partial S_{1}
$$

Again, we integrate the boundary conditions over $\partial S_{1}$, but now we study how the new term $\vec{v}_{0}$ behaves inside the integral. 
Expanding $\vec{v}_{0}$ as a Taylor series, terms of odd order do not contribute to the integral due to the spherical symmetry of $\partial S$. It can furthermore be shown in a lengthy but straightforward calculation (see pages 254-255 in [88]) which we omit here, that terms of even order higher than two vanish for all flow fields obeying the creeping flow equations.

Keeping only the zeroth and second order term, we can hence update Stokes law (equation 2.15) with two new terms introduced by the given velocity field $\vec{v}_{0}$ as follows:

$$
\vec{v}_{1}=\frac{1}{\gamma} \vec{F}_{1}+\left(1+\frac{a^{2}}{6} \vec{\nabla}^{2}\right)_{\vec{r}=\vec{r}_{1}} \vec{v}_{0}
$$

This equation is called Faxén's law for translational motion. For $\vec{v}_{0}=0$ it immediately reduces to the Stokes law. We treat $\vec{v}_{0}$ and the force $\vec{F}_{1}$ acting on the sphere as given and use Faxén's law to determine the sphere's resulting velocity $\vec{v}_{1}$. Naturally, Faxén's law can also be used the other way around - it determines $\vec{F}_{1}$ when $\vec{v}_{1}$ is known.

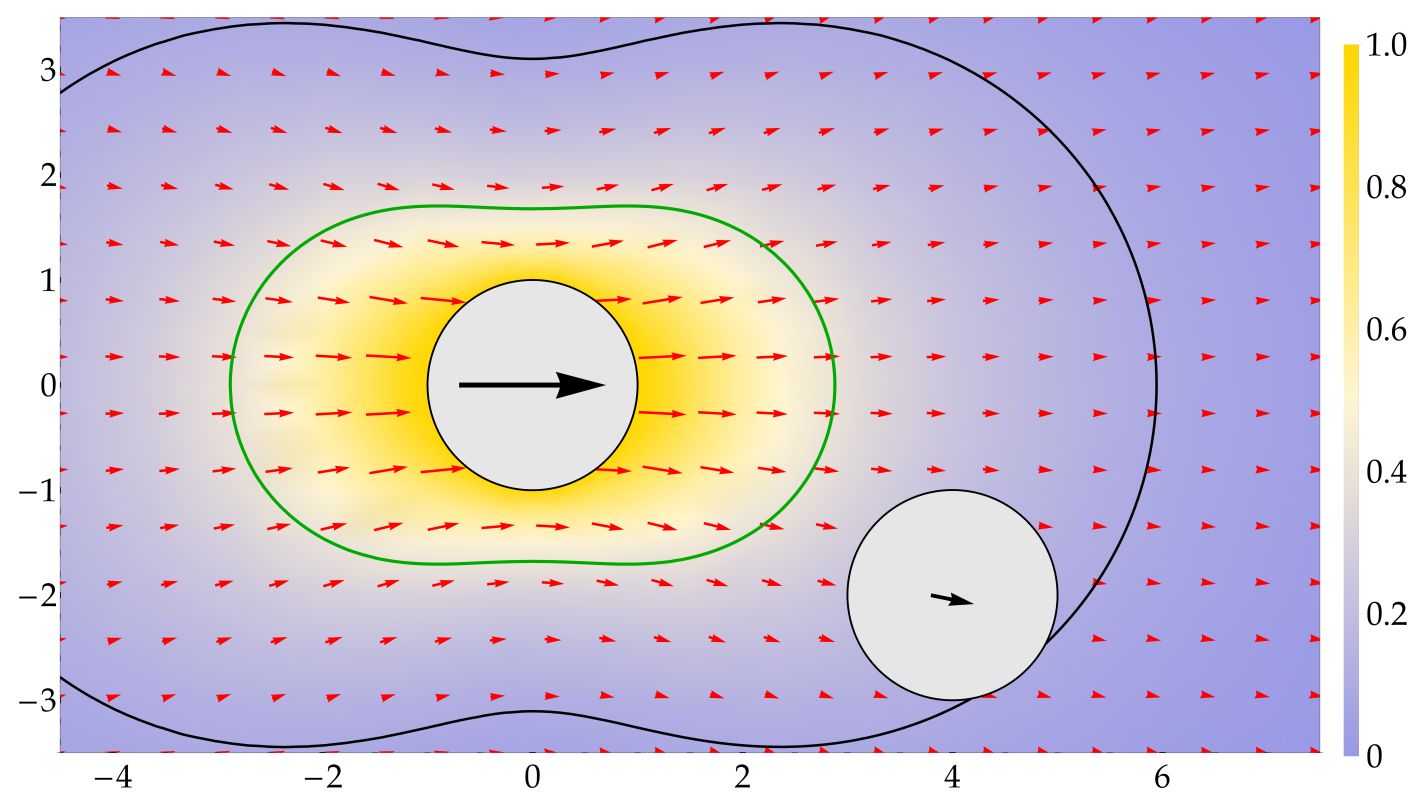

Figure 2.1: The velocity field (red arrows) around a unit sphere (origin) moving with unit speed in $\mathrm{x}$-direction as given by equation 2.17. The background color shows its absolute value in terms of the sphere's velocity. The closed lines show contour lines of the velocity field's absolute value. At the inner green line, the velocity has dropped to $50 \%$, and at the outer black line it has dropped to $25 \%$. A second sphere (bottom right) is embedded in the velocity field caused by the first sphere. It moves along with the fluid flow around it - this effect is called a "hydrodynamic interaction". Its velocity can be quantified using Faxén's law (equation 2.22), or equivalently via the Rotne-Prager matrices (equation 2.29). The velocities of both spheres are indicated with black arrows at their respective centers. The lengths of these arrows have the correct ratio, but are not in the proper scale with the fluid flow for visual purposes. The fluid velocity equals that of the first sphere at its boundary. 
Two spheres in a Stokes flow Let us now consider two spheres $S_{1}$ and $S_{2}$ with radii $a_{1}$ and $a_{2}$, center points $\vec{r}_{1}$ and $\vec{r}_{2}$, translational velocities $\vec{v}_{1}$ and $\vec{v}_{2}$, rotational velocities $\vec{\omega}_{1}$ and $\vec{\omega}_{2}$, and forces $\vec{F}_{1}$ and $\vec{F}_{2}$ acting on them in a Stokes flow which would be at rest in the absence of the spheres (see figure 2.1 ). The no-slip boundary conditions for the fluid flow $\vec{v}(\vec{r})$ now read

$$
\begin{array}{llll} 
& \vec{v}_{1}+\vec{\omega}_{1} \times\left(\vec{r}-\vec{r}_{1}\right)=\vec{v}(\vec{r}) & \text { where } & \vec{r} \in \partial S_{1} \\
\text { and } \quad & \vec{v}_{2}+\vec{\omega}_{2} \times\left(\vec{r}-\vec{r}_{2}\right)=\vec{v}(\vec{r}) & \text { where } & \vec{r} \in \partial S_{2}
\end{array}
$$

and the force density is given by

$$
\vec{f}(\vec{r})=\frac{1}{4 \pi a_{1}} \delta\left(\left|\vec{r}-\vec{r}_{1}\right|-a_{1}\right) \vec{F}_{1}+\frac{1}{4 \pi a_{2}} \delta\left(\left|\vec{r}-\vec{r}_{2}\right|-a_{2}\right) \vec{F}_{2}
$$

Even for only two spheres of equal size, this boundary value problem for the creeping flow equations does not have a closed analytical solution. The solution can be constructed, however, using an iterative method which is called the method of reflections. It can be found e.g. in chapter 5.12 of the textbook by Dhont [88], but exceeds the scope of this thesis. The method of reflections is in principle straightforward for two spheres. For three spheres, only the leading order terms in a series expansion of the correct flow field are known, and for more accuracy or more spheres one needs to retreat to expensive numerics. This makes the method of reflections impractical for most applications, including ours, which is why we do not discuss it in detail ${ }^{1}$. Instead, we find an approximate solution to the given boundary value problem.

\subsubsection{Rotne-Prager approximation}

Let us now consider $N$ spheres numbered $k=1, \ldots, N$. Even in this case, we can expect a linear relation between the spheres' velocities and the forces acting on them due to the linearity of the creeping flow equations. Defining the collection of velocities $\vec{V}:=\left(\vec{v}_{1}, \ldots, \vec{v}_{N}\right)^{T}$ and the collection of forces $\vec{F}$ analogously, one may therefore write

$$
\begin{aligned}
\vec{V} & =\boldsymbol{\mu} \cdot \vec{F} \\
\vec{v}_{i} & =\sum_{j=1}^{N} \boldsymbol{\mu}_{i j} \cdot \vec{F}_{j} .
\end{aligned}
$$

This is the defining equation for the $3 N \times 3 N$-dimensional mobility matrix $\boldsymbol{\mu}$, which consists of $N^{2} 3 \times 3$-blocks $\boldsymbol{\mu}_{i j}$ (see also equation 2.30 ).

\footnotetext{
${ }^{1}$ In a nutshell, one finds the required solution $\vec{v}(\vec{r})$ of the boundary value problem 2.23 as a series expansion. The first term in this series is the velocity field $\vec{v}^{(0)}$ that sphere $i$ causes in absence of sphere $j$, see equation $2.17 . \vec{v}^{(0)}$ obeys the boundary conditions on sphere $i$. Next, one embeds sphere $j$ in the flow field $\vec{v}^{(0)}$ using Faxén's law and determines the additional flow field $\vec{v}^{(1)}$ sphere $j$ causes on top of $\vec{v}^{(0)} \cdot \vec{v}^{(1)}$ obeys the boundary conditions on sphere $j$. This additional flow field is then used to consider sphere $i$ again in order to calculate a flow field $\vec{v}^{(2)}$ which is more accurate than $\vec{v}^{0}$ and obeys the boundary conditions on sphere $i$. Next, sphere $j$ is considered again in the flow field $\vec{v}^{(2)}$ and so forth.
} 
Note that due to the Stokes law, the "self mobility matrix" of a sphere with radius $a$ is already known to us: it is $\boldsymbol{\mu}_{i i}=\gamma^{-1} \mathfrak{1}_{3 \times 3}$. The off-diagonal matrices $\boldsymbol{\mu}_{i j}$ describe how the velocity of sphere $i$ is affected by the force acting on sphere $j$, which is called a hydrodynamic interaction. Without hydrodynamic interactions, $\boldsymbol{\mu}$ would therefore be diagonal.

The most commonly used approximation that enables us to calculate closed analytical expressions for all mobility matrices is called the Rotne-Prager approximation [52, 53]. Let us consider the mobility matrix $\boldsymbol{\mu}_{i j}$, i.e. the influence the force $\vec{F}_{j}$ acting on sphere $j$ has on the velocity $\vec{v}_{i}$ of sphere $i$. This situation is visualized in figure 2.1 - here, we are calculating the bottom right sphere's velocity. The Rotne-Prager approximation is twofold:

1. We ignore all other spheres which are neither $i$ nor $j$.

2. We assume that sphere $i$ experiences the flow field which sphere $j$ would cause in the absence of sphere $i$.

Using these assumptions, the situation at hand is the following: Sphere $j$ experiences a force acting on it and thus causes the velocity field $\vec{v}^{(j)}(\vec{r})$ given by equation 2.17 . This velocity field solves the creeping flow equations and fulfills the boundary conditions on sphere $j$. Next we embed sphere $i$ in this velocity field and immediately make use of Faxén's law (equation 2.22) to find its velocity $\vec{v}_{i}$. This procedure yields only an approximation to the true solution for two spheres because the flow field $\vec{v}^{(j)}(\vec{r})$ ignores the boundary conditions on sphere $i$.

Within the Rotne-Prager approximation, the velocity of sphere $i$ due to the force on sphere $j$ is thus

$$
\begin{aligned}
\vec{v}_{i} & =\left(1+\frac{a_{i}^{2}}{6} \vec{\nabla}^{2}\right)_{\vec{r}=\vec{r}_{i}} \vec{v}^{(j)}(\vec{r}) \\
& =\frac{1}{4 \pi a_{i}^{2}} \int_{\partial S_{i}} \vec{v}^{(j)}(\vec{r}) \mathrm{d} A \\
& =\frac{1}{4 \pi a_{i}^{2}} \int_{\partial S_{i}} \int_{\partial S_{j}} \boldsymbol{T}\left(\vec{r}-\vec{r}^{\prime}\right) \frac{1}{4 \pi a_{j}^{2}} \vec{F}_{j} \mathrm{~d} A^{\prime} \mathrm{d} A
\end{aligned}
$$

Comparing this with equation 2.25 , we read off that

$$
\boldsymbol{\mu}_{i j}=\frac{1}{4 \pi a_{i}^{2}} \frac{1}{4 \pi a_{j}^{2}} \int_{\partial S_{i}} \int_{\partial S_{j}} \boldsymbol{T}\left(\vec{r}-\vec{r}^{\prime}\right) \mathrm{d} A \mathrm{~d} A^{\prime},
$$

which is invariant under exchanging $i$ and $j$ :

$$
\boldsymbol{\mu}_{i j}=\boldsymbol{\mu}_{j i}
$$

From a physical perspective, this symmetry is remarkable: Pulling on sphere $i$ affects sphere $j$ in the same way as pulling with the same force on sphere $j$ affects sphere $i$. 
From a mathematical perspective, we have at hand pairwise interactions of surface elements on the two spheres, which are mediated via the underlying symmetric Green's function $\mathbf{T}(\vec{x})=\mathbf{T}(-\vec{x})$. The expressions above are also valid for overlapping spheres: For physical intuition, note that the flow field $\vec{v}^{(j)}(\vec{r})$ (according to equation 2.17) exists even inside of sphere $j$ where it has the constant flow velocity $\vec{v}_{j}$. Sphere $i$ is embedded in this field at an arbitrary position, which requires a case distinction upon solving the integrals above, depending on whether or not parts of $\partial S_{i}$ lie within $S_{j}$.

The double integrals can be solved analytically, which we do not demonstrate here for obvious reasons - they can be found in references [54, 55] for potentially overlapping and different-sized spheres. Defining $\vec{r}_{i j}=\vec{r}_{i}-\vec{r}_{j}$ and $r_{i j}=\left|\vec{r}_{i j}\right|$, the final expressions we require in this thesis read

$$
\boldsymbol{\mu}_{i j}= \begin{cases}\frac{1}{8 \pi \eta r_{i j}} \cdot\left[\left(1+\frac{a_{i}^{2}+a_{j}^{2}}{3 r_{i j}^{2}}\right) \mathbb{1}_{3 \times 3}+\left(1-\frac{a_{i}^{2}+a_{j}^{2}}{r_{i j}^{2}}\right) \frac{\vec{r}_{i j} \cdot \vec{r}_{i j}^{T}}{r_{i j}^{2}}\right] & \text { if } \quad a_{i}+a_{j}<r_{i j}, \\ \frac{1}{6 \pi \eta a_{i} a_{j}} \cdot\left[\frac{16 r_{i j}^{3}\left(a_{i}+a_{j}\right)-\left(\Delta a^{2}+3 r_{i j}^{2}\right)^{2}}{32 r_{i j}^{3}} \mathbb{1}_{3 \times 3}+\frac{3\left(\Delta a^{2}-r_{i j}^{2}\right)^{2}}{32 r_{i j}^{3}} \frac{\vec{r}_{i j} \cdot \vec{r}_{i j}^{T}}{r_{i j}^{2}}\right] & \text { if } \quad \Delta a<r_{i j} \leq a_{i}+a_{j} \\ \frac{1}{6 \pi \eta a_{>}} \mathbb{1}_{3 \times 3} & \text { if } \quad r_{i j} \leq \Delta a \quad,\end{cases}
$$

where $a_{<}\left(a_{>}\right)$is the smaller (larger) value among $a_{i}$ and $a_{j}$, and $\Delta a=a_{>}-a_{<}$. Note that only the first line is required for non-overlapping spheres and that the expressions further simplify for equally sized spheres. As may be expected, $\boldsymbol{\mu}_{i j}$ depends only on the vector $\vec{r}_{i j}$. For large distances $r_{i j}$ between the two spheres (or for $a \rightarrow 0$ ) it converges towards the Oseen-tensor. This makes sense from a physical perspective as the two spheres will then perceive each other as mere points. In fact, the Rotne-Prager mobility matrices can be regarded as a higher-order correction to the Oseen-tensor, taking into account the beads' finite sizes. As a side note, the method of reflections we mentioned earlier delivers further correction terms which are of higher order in $a / r_{i j}$ than the mobility matrices we are using. However, to my knowledge, these higher order terms are not known in closed form for different sized, overlapping spheres.

For later use in this thesis, let us define the $3 N \times 3 N$-dimensional diffusion matrix $\mathbf{D}$ which consists of $N^{2} 3 \times 3$-blocks $\mathbf{D}_{i j}=T \boldsymbol{\mu}_{i j}$ where $T$ is the surrounding fluid's temperature (not the Oseen matrix!):

$$
\mathbf{D}=T \cdot\left(\begin{array}{cccc}
\boldsymbol{\mu}_{11} & \boldsymbol{\mu}_{12} & \cdots & \boldsymbol{\mu}_{1 N} \\
\boldsymbol{\mu}_{21} & \boldsymbol{\mu}_{22} & & \vdots \\
\vdots & & \ddots & \\
\boldsymbol{\mu}_{N 1} & \cdots & & \boldsymbol{\mu}_{N N}
\end{array}\right)
$$

Note that $\mathbf{D}$ is symmetric. 


\subsubsection{Hydrodynamics near a wall}

So far, we have considered hydrodynamics in an infinite medium (bulk). In some applications, the presence of a wall is relevant, e.g. when a molecule is bound to a surface (see section 5.4, page 133). All concepts we have shown are formally universal. The only changes required when a wall is present is to take additional no-slip boundary conditions at the wall into account. In particular, these boundary conditions alter the Green's functions $\boldsymbol{T}(\vec{r})$ and $\vec{g}(\vec{r})$ of the creeping flow equations. The Green's function $\boldsymbol{T}^{\text {wall }}(\vec{r})$ for the velocity field near a wall is called the Blake tensor [93]. For physical intuition, figure 2.2 shows a sketch of the velocity caused by a sphere moving away from a wall.

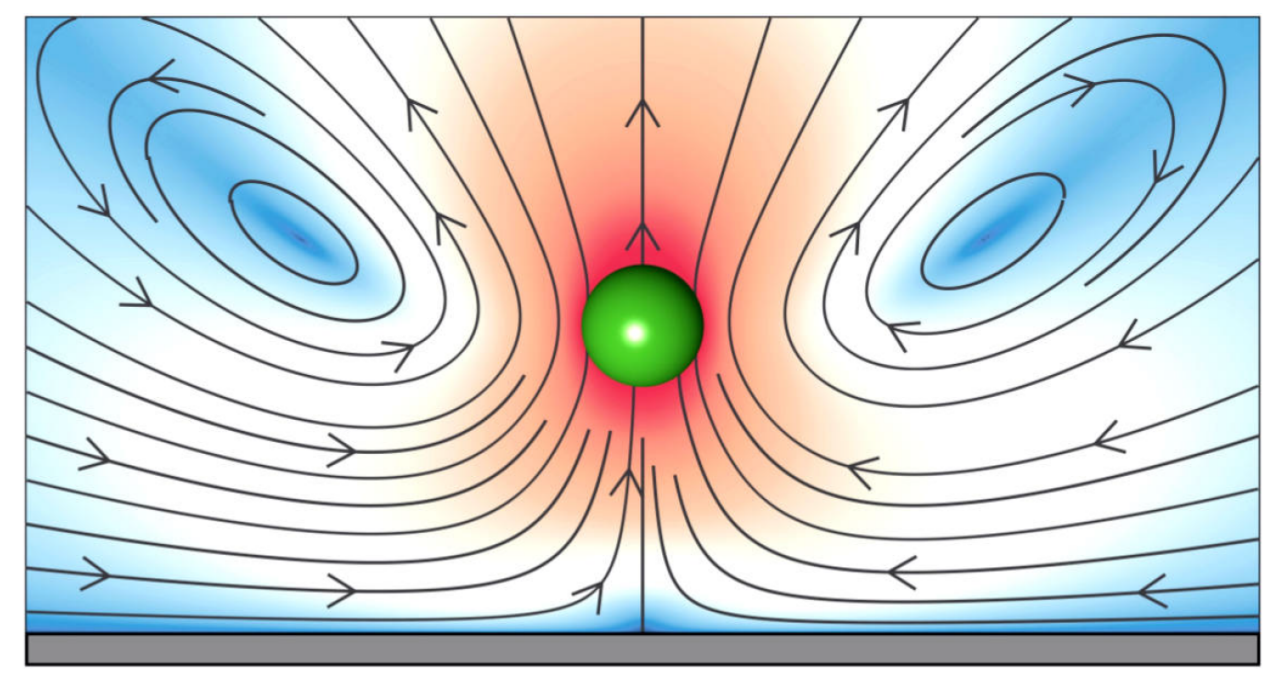

Figure 2.2: A 2-dimensional cross section of the flow field near a wall when a sphere is moving away from it. This figure was kindly provided by Rajesh Singh and published in [94].

The Rotne-Prager approximation next to a wall is considered in references [94-96], and the mobility matrices read

$$
\boldsymbol{\mu}_{i j}^{(\text {wall })}\left(\vec{r}_{i}, \vec{r}_{j}\right)=\left\{\begin{array}{ll}
\boldsymbol{\mu}_{i}^{\text {self }}\left(z_{i}\right) & \text { if } i=j \\
\boldsymbol{\mu}_{i j}\left(\vec{r}_{i}-\vec{r}_{j}\right)-\boldsymbol{\mu}_{i j}\left(\vec{r}_{i}-\overline{\vec{r}}_{j}\right)+\hat{\boldsymbol{\mu}}\left(\vec{r}_{i}, \vec{r}_{j}\right) & \text { if } i \neq j
\end{array} .\right.
$$


Here it is assumed that the wall lies in the $\mathrm{x}$-y-plane and that the spheres do not overlap with the wall. Moreover, the mirrored coordinate $\overline{\vec{r}}_{j}=\left(x_{j}, y_{j},-z_{j}\right)^{T}$ and the matrix $\hat{\boldsymbol{\mu}}\left(\vec{r}_{i}, \vec{r}_{j}\right)$ were defined as

$$
\begin{aligned}
\hat{\boldsymbol{\mu}}_{\alpha, \alpha} & =\frac{1}{4 \pi \eta}\left(\frac{-z_{i} z_{j}}{R^{3}}\left(1-3 \frac{R_{\alpha}^{2}}{R^{2}}\right)+\frac{a^{2} R_{3}^{2}}{R^{5}}\left(1-5 \frac{R_{\alpha}^{2}}{R^{2}}\right)\right) \\
\hat{\boldsymbol{\mu}}_{\alpha, \beta} & =\frac{1}{4 \pi \eta}\left(\frac{3 z_{i} z_{j} R_{\alpha} R_{\beta}}{R^{5}}-5 a^{2} \frac{R_{\alpha} R_{\beta} R_{3}^{2}}{R^{7}}\right) \\
\hat{\boldsymbol{\mu}}_{\alpha, 3} & =\frac{1}{4 \pi \eta}\left(\frac{z_{j} R_{\alpha}}{R^{3}}\left(1-3 \frac{z_{i} R_{3}}{R^{2}}\right)-\frac{a^{2} R_{\alpha} R_{3}}{R^{5}}\left(2-5 \frac{R_{3}^{2}}{R^{2}}\right)\right) \\
\hat{\boldsymbol{\mu}}_{3, \alpha} & =\frac{1}{4 \pi \eta}\left(\frac{z_{j} R_{\alpha}}{R^{3}}\left(1+3 \frac{z_{i} R_{3}}{R^{2}}\right)-5 \frac{a^{2} R_{\alpha} R_{3}^{3}}{R^{7}}\right) \\
\text { and } \quad \hat{\boldsymbol{\mu}}_{3,3} & =\frac{1}{4 \pi \eta}\left(\frac{z_{i} z_{j}}{R^{3}}\left(1-3 \frac{R_{3}^{2}}{R^{2}}\right)-\frac{a^{2} R_{3}^{2}}{R^{5}}\left(3-5 \frac{R_{z}^{2}}{R^{2}}\right)\right)
\end{aligned}
$$

where $\vec{R}=\vec{r}_{i}-\overline{\vec{r}}_{j}, R=|\vec{R}|$ and $\alpha, \beta \in\{1,2\}$. The self-mobility matrices read

$$
\mu_{i}^{\text {self }}=\left(\begin{array}{ccc}
\mu_{\|}\left(z_{i}\right) & 0 & 0 \\
0 & \mu_{\|}\left(z_{i}\right) & 0 \\
0 & 0 & \mu_{\perp}\left(z_{i}\right)
\end{array}\right)
$$

where

$$
\begin{aligned}
\mu_{\|} & =\left(1-\frac{9 a}{16 z}+\frac{1}{8}\left(\frac{a}{z}\right)^{3}+\mathscr{O}\left(\frac{a}{z}\right)^{5}\right) / \gamma \\
\text { and } \quad \mu_{\perp} & =\left(1-\frac{9 a}{8 z}+\frac{1}{2}\left(\frac{a}{z}\right)^{3}+\mathscr{O}\left(\frac{a}{z}\right)^{5}\right) / \gamma \quad .
\end{aligned}
$$

We observe that in the presence of a wall, the mobility matrices no longer depend only on the vector $\vec{r}_{i}-\vec{r}_{j}$, but on both $\vec{r}_{i}$ and $\vec{r}_{j}$ individually, which makes sense, as information where the spheres are placed in relation to the wall is required. Even the self-mobility matrices require the distance of the sphere from the surface and show that a single sphere's movement is altered next to a wall due to the additional no-slip boundary condition. 


\subsection{Statistical mechanics}

Statistical mechanics is the application of probability theory and the theory of stochastic processes to physical systems. We make use of it to derive the forces acting on the beads considered in the previous section and thereby arrive at their stochastic equation of motion, which is called the Langevin equation. In contrast to quantum mechanics, the reason why randomness plays a role in statistical mechanics is not because it is an inherent property of the physical processes. Its origin is instead a result of our practical viewpoint; although we have an enormous number of degrees of freedom at hand, we explicitly study only a few of them (the "system") and choose to treat the remaining ones (the "heat bath") in a statistical manner only. The laws of statistics are, however, powerful and universal, and their implications on physics are vast. As any physical theory does, statistical mechanics requires axioms from which to start our calculations. A hallmark of statistical mechanics is that one can view and understand it from many different viewpoints which in the end all turn out to be equivalent. Often, there is no consensus if statement $A$ should be the axiom from which statement $B$ follows, or vice versa - in a sense it is a matter of taste. For instance, one can equivalently claim that all microstates in a closed system with fixed energy must be equally probable, and then deduce that this probability distribution maximizes entropy, or one can use entropy maximization as an axiom and then deduce that all states are equally probable. The same holds true for the Langevin equation - it can be derived from the FokkerPlanck equation; or it can be constructed in its own right and the Fokker-Planck equation be derived from it. In the following, we first consider the thermodynamics of the canonical ensemble, then derive the Fokker-Planck equation by combining thermodynamics with hydrodynamics and probability theory, and finally deduce the Langevin equation from it.

Many introductory level textbooks on thermodynamics and statistical mechanics are available. For the more advanced topics treated here, the textbooks by Risken [97], Gardiner [1], van Kampen [98] and Mazo [99] can be recommended. Unless stated otherwise, I have mainly followed the reasoning found in the lecture script by Garcia [100] and Schön [101].

\subsubsection{Physics of the canonical ensemble}

Let us consider a system whose state is represented by the abstract variables $\vec{x} \in \Omega$ embedded in a heat bath. Imagine, for example, a discrete chain swimming in water at finite temperature $T$, such that $\Omega$ is the set of all $3 N$-dimensional chain conformations (phase space). The system also possesses an energy function $E(\vec{x})$ (e.g. reflecting its elastic properties). This situation is known in thermodynamics as the canonical ensemble: the system of interest is coupled to a heat bath and is allowed to exchange heat with it, making its total energy and momentum fluctuating rather than preserved quantities. 
When the microstate of a system is prepared to be $\vec{x}_{0}$ at time zero, all information about it is initially available: the probability distribution for $\vec{x}$ is a delta function: $p(\vec{x}, 0)=\delta\left(\vec{x}-\vec{x}_{0}\right)$. For times $t>0$, the system's current microstate $\vec{x}$ is no longer known since its evolution is stochastic. We imagine an ensemble of trajectories $\vec{x}(t)$ : A set of infinitely many independent realizations of the experiment obeying the initial condition. The trajectories in the ensemble independently branch out from the initial condition, and to an outside observer who went for lunch after preparing the initial condition, the particular stochastic trajectory the system has chosen is unknown. Therefore, its current state can then only be quantified in terms of a probability distribution $p(\vec{x}, t)$. This distribution is best understood as a histogram over all microstates in the ensemble at time $t$. For intuition, compare figures 2.4 (page 44) which shows trajectories in an ensemble, and figure 2.3 (page 40) which shows the corresponding probability distributions at different times.

Entropy We start our calculations with the following axiom: Sufficiently long after knowledge about the system's initial state is available, $p(\vec{x})$ has relaxed towards a stationary state which has maximum uncertainty $S[p]$. This situation is referred to as thermal equilibrium. Thanks to the groundbreaking work of Claude Shannon [102], the founder of information theory, the somewhat vague concept of uncertainty can be mathematically quantified by the probability distribution's entropy $S$. It can in fact be shown (see e.g. pages 68-71 in [103]) that the functional $S$ is uniquely determined from the three axioms that (i) $S$ be a real, continuous functional of $p$, (ii) if $p$ is constant, say $p_{i}=\frac{1}{N}$, then $S$ be an increasing function of $N$, and (iii) $S$ be additive under groupings of the states $\vec{x}$ in $\Omega$. The third axiom makes sure that the uncertainties on distinct regions in $\Omega$ add up when they are treated as a whole: uncertainties about independent systems must be additive. Up to an arbitrary prefactor which we set to 1 , the only function with these properties is known as entropy, and it reads

$$
S[p]=-\langle\ln p\rangle=-\int_{\Omega} p(\vec{x}) \ln p(\vec{x}) \mathrm{d} \vec{x} \quad .
$$

Note that contrary to entropy in conventional thermodynamics, this entropy is dimensionless, which corresponds to setting Boltzmann's constant to $k_{B}=1$. This definition of entropy is also known as the Gibbs entropy: $p(\vec{x})$ arises from an ensemble rather than from a time average.

Technically speaking, Shannon's axioms above only lead to a discrete version ("Shannon entropy") of the Gibbs entropy. Taking its continuum limit is not trivial, and requires singling out a set of variables $\vec{x}$ with respect to which $p(\vec{x})$ shall be maximally ignorant. For instance, a uniform distribution on a spherical surface ${ }^{2}$ is constant with respect to the variables $(\phi, \cos \theta)$ but not with respect to $(\phi, \theta)$. We must thus keep in mind that $\vec{x}$ in our entropy definition represents Cartesian coordinates. For technical details on this issue, we refer to chapters 4.5 and 5.2 in reference [103].

\footnotetext{
${ }^{2}$ For a uniform distribution on the surface of a unit sphere, surface elements carry a probability $\propto|\mathrm{d} A|=$ $|\sin \theta \mathrm{d} \theta \mathrm{d} \phi|=|\mathrm{d}(\cos \theta) \mathrm{d} \phi|$. Therefore, $\cos \theta$ and not $\theta$ is uniformly distributed.
} 
Boltzmann distribution At the same time as maximizing its entropy, it is required that $p$ fulfills two constraints. Firstly, any probability distribution must be normalized such that $\int_{\Omega} p(\vec{x}) \mathrm{d} \vec{x}=1$. Secondly, the average energy $E$ of the chain cannot be unbounded, but the heat bath dictates some fixed average value $U=\langle E\rangle$ which is called its inner energy. Let us now find the function $p(\vec{x})$ which maximizes its entropy while fulfilling these two constraints. The variational problem at hand can be solved by introducing two scalar Lagrangian multipliers $\alpha$ and $\beta$ representing the constraints, and solving

$$
\begin{aligned}
0 & \stackrel{!}{=} \delta\left(S[p]-\alpha \cdot\left(\int_{\Omega} p(\vec{x}) \mathrm{d} \vec{x}-1\right)-\beta \cdot\left(\int_{\Omega} p(\vec{x}) E(\vec{x}) \mathrm{d} \vec{x}-U\right)\right) \\
& =\int_{\Omega} \delta(-p \ln p-\alpha p-\beta p E) \mathrm{d} \vec{x} \\
& =\int_{\Omega} \delta p \cdot(-\ln p-1-\alpha-\beta E) \mathrm{d} \vec{x}
\end{aligned}
$$

Setting the integrand to zero yields

$$
\begin{aligned}
0 & =-\ln p-1-\alpha-\beta E \\
\Leftrightarrow \quad p(\vec{x}) & =\frac{1}{Z} \exp (-\beta E)
\end{aligned}
$$

where we redefined $Z^{-1}=\exp (-\alpha-1) . Z$ is called the partition sum and plays the role of a normalization factor of $p$. It can hence be determined from the normalization constraint and is given by

$$
Z=\int_{\Omega} \exp (-\beta E) \mathrm{d} \vec{x}
$$

The second Lagrangian multiplier, $\beta$, can be interpreted as follows. Since the argument of the exponential function must be dimensionless, $\beta^{-1}$ must be an energy. It determines the energy scale on which the probability to find the system in a certain state $\vec{x}$ decays. For $\beta \rightarrow \infty$, states with more energy than the minimum energy the system can take on do not carry any statistical weight. For $\beta \rightarrow 0$, all states carry the same statistical weight, regardless of their energy. At the same time, the system's inner energy $U$ can be tuned by changing $\beta$ because

$$
U \propto \int_{\Omega} E \cdot \exp (-\beta E) \mathrm{d} \vec{x} \quad
$$

It would be perfectly reasonable to treat $\beta$ as a free parameter characterizing the heat bath that the system is embedded in. It is more intuitive, however, to deal with the energy scale $\beta^{-1}$ instead, which is better known as the temperature

$$
T=\beta^{-1} \quad .
$$

The fact that the temperature is an energy is a result of our convention that $k_{B}=1$. 
The probability distribution therefore takes on the simple form

$$
p(\vec{x})=\frac{1}{Z} \exp \left(-\frac{E(\vec{x})}{T}\right),
$$

which is called the Boltzmann distribution. Note that it is entirely characterized by the system's energy landscape $E$ and the heat bath's temperature $T$ - no kinematic details enter it. For instance, whether or not all vertices (beads) of the chain have identical friction coefficients and whether or not hydrodynamic interactions are considered for the chain's dynamics does not alter the Boltzmann distribution, which is quite remarkable. The Boltzmann distribution has two further properties which are sometimes even used to derive it in a heuristic manner. Firstly, due to being normalized, it does not change when a constant is added to the energy function $E^{\prime}(\vec{r})=E(\vec{r})+$ const., as it should not because only the gradients of potential functions have physical relevance. Secondly, when two non-interacting physical systems with energy functions $E_{1}\left(\vec{x}_{1}\right)$ and $E_{2}\left(\vec{x}_{2}\right)$ are treated as one global system with $E=E_{1}+E_{2}$, then the probability distribution for the global system factorizes $\left(p\left(\vec{x}_{1}, \vec{x}_{2}\right) \propto \exp \left(-E_{1} / T\right) \cdot \exp \left(-E_{2} / T\right)\right)$, which correctly demonstrates the statistical independence of the two subsystem's states.

Another side note is that the celebrated equipartition theorem directly follows from the Boltzmann distribution: Consider any variable $q$ which enters the system's energy function in a quadratic manner (e.g. a harmonic elastic potential, or a particle's velocity) such that $E=\frac{c}{2} \cdot q^{2}$. Then its probability distribution is the Gaussian

$$
p(q) \propto \exp \left(-\frac{c q^{2}}{2 T}\right) \quad,
$$

and we can immediately read off its variance to be

$$
\left\langle q^{2}\right\rangle=\frac{T}{c}
$$

This gives the average energy in q's degree of freedom:

$$
U=\langle E\rangle=\left\langle\frac{c}{2} q^{2}\right\rangle=\frac{T}{2}
$$

This is yet another perspective which allows us to interpret the Lagrangian multiplier we named temperature as an energy scale relevant to the physical system.

Free energy From equation 2.36 we observe that, within the space of all normalized probability distributions, the Boltzmann distribution minimizes the functional

$$
F=U-T S,
$$

which is called the (Helmholtz) free energy. It is the minimized thermodynamic potential in the canonical ensemble. 
The distinction between entropy maximization and free energy minimization is entirely formal: the latter considers the system's inner energy $U$ as part of the extremized functional, while the former uses its value solely as a mathematical constraint. Both perspectives consider the normalization of $p$ as a mathematical constraint. Taking the viewpoint of free energy minimization, some physical insight is gained: We have at hand a balancing act between two contradicting extreme cases: minimizing energy (causing a sharply peaked probability distribution) and maximizing entropy (causing a uniform probability distribution), with the temperature acting as a weighting factor dictating which of these two extremes enjoys higher priority.

Inserting the Boltzmann distribution into the definition of entropy (equation 2.35) yields

$$
\begin{aligned}
S & =-\int_{\Omega} p \ln p \mathrm{~d} \vec{x} \\
& =-\int_{\Omega} p \ln \left(\frac{1}{Z} \exp \left(-\frac{E}{T}\right)\right) \mathrm{d} \vec{x} \\
& =-\int_{\Omega} p \cdot\left(-\ln Z-\frac{E}{T}\right) \mathrm{d} \vec{x} \\
& =\ln Z+\frac{U}{T},
\end{aligned}
$$

which (upon comparison with equation 2.45) shows us that the system's free energy is given by

$$
F=-T \ln Z \quad .
$$

Finally, we consider a subset $\Omega_{A}$ of $\Omega$ : a mesostate $A$. For example, if $\Omega$ is the set of all conformations (microstates) of a polymer, then $\Omega_{A}$ (the set of all open states) could be the set of all conformations for which the end-to-end distance of the polymer exceeds some given value.

The total probability to find the system in $\Omega_{A}$ is given by

$$
p(A)=\int_{\Omega_{A}} p \mathrm{~d} \vec{x}=\frac{1}{Z} \int_{\Omega_{A}} \exp \left(-\frac{E}{T}\right) \mathrm{d} \vec{x}=: \frac{Z_{A}}{Z} \quad .
$$

Thus, the partition function $Z$ denotes the total probability of the set of microstates $\vec{x}$ over which $p$ was integrated. In analogy to the probability of a microstate, which is given by the Boltzmann distribution with that microstate's energy in the exponent, we may therefore write

$$
p(A)=\frac{1}{Z} \exp \left(-\frac{F_{A}}{T}\right)
$$

where $F_{A}=-T \ln Z_{A}$ is the free energy of the mesostate $A$. The relative probabilities of microstates $\vec{x}_{1}$ and $\vec{x}_{2}$, and of mesostates $A$ and $B$ are therefore given by Boltzmann factors

$$
\text { and } \begin{aligned}
\frac{p\left(\vec{x}_{1}\right)}{p\left(\vec{x}_{2}\right)} & =\exp \left(-\frac{E\left(\vec{x}_{1}\right)-E\left(\vec{x}_{2}\right)}{T}\right) \\
\frac{p(A)}{p(B)} & =\exp \left(-\frac{F_{A}-F_{B}}{T}\right) .
\end{aligned}
$$

In summary, one may hence interpret the free energy as the effective energy associated with a mesostate (or $\Omega$ itself) rather than a microstate. The probability distribution for microstates hidden within that mesostate minimizes its free energy. 
Detailed balance Note that, by construction, all the arguments above include no mention of time. That is because we just studied statistical properties of a physical system in thermal equilibrium, which is well known as classical thermodynamics. In my opinion, this terminology is very unfortunate as thermodynamics explicitly does not concern itself with dynamics. We must bear in mind, however, that even in thermal equilibrium, a physical system constantly evolves over time. It changes its microstate $\vec{x}$, sampling the Boltzmann distribution as its time average. While the statistical properties of the system are easily found - only the energy function $E(\vec{x})$ is required - examining its dynamical properties (anything requiring a notion of time) is far from trivial. Consider, for example, the fact that the sampled probability distribution of a sufficiently long trajectory $\vec{x}(t)$ does not change when it is sped up or slowed down, or when it is cut into parts which are then reassembled such that $\vec{x}(t)$ may perform discontinuous jumps.

To study dynamics in thermal equilibrium, we require another physical axiom other than free energy minimization (determining all statistical properties), and this is called the detailed balance condition. It states that a sufficiently long time after $\vec{x}$ was prepared to obey some known initial condition, the trajectories $\vec{x}(t)$ obey time-reversal symmetry. For instance, this means that there is no way of telling if a video of a polymer chain in thermal equilibrium is played forward or backward. This is a stronger requirement than stating that the probability distribution $p(\vec{x})$ be stationary. Consider for example a three-state system $\Omega=\left\{x_{1}, x_{2}, x_{3}\right\}$ whose dynamics cyclically move from states $x_{1} \rightarrow x_{2} \rightarrow x_{3} \rightarrow x_{1} \rightarrow x_{2} \rightarrow \ldots$ with unit speed - these trajectories sample a stationary distribution but do not obey time reversal symmetric. The detailed balance condition must hold for any two states $x_{i}$ and $x_{j}$ :

$$
\begin{gathered}
\text { probability flux }\left(x_{i} \rightarrow x_{j}\right)=\operatorname{probability~flux}\left(x_{j} \rightarrow x_{i}\right) \\
\begin{aligned}
p\left(x_{i}\right) \cdot k\left(x_{i}, x_{j}\right) & =p\left(x_{j}\right) \cdot k\left(x_{j}, x_{i}\right) \\
\Leftrightarrow \quad \frac{k\left(x_{i}, x_{j}\right)}{k\left(x_{j}, x_{i}\right)} & =\exp \left(-\frac{E\left(\vec{x}_{j}\right)-E\left(\vec{x}_{i}\right)}{T}\right)
\end{aligned}
\end{gathered}
$$

where $k(p, q)$ is the transition rate per time from state $p$ to state $q$.

\subsubsection{Basic notions of probability theory}

This section presents textbook knowledge about probability theory which can also be found in e.g. the textbook by Gardiner [1].

Notation We consider the temporal evolution of a random variable $X$ which takes on values $x(t) \in \Omega$ at times $t>0$, and we use the convention that $0=t_{0} \leq t_{1} \leq t_{2} \leq \ldots$. The set $\Omega$ is called the phase space and the collection of the values $\{x(t)\}_{t>0}$ is called a trajectory, which is one particular realization of the stochastic process $X(t)$. 
An infinite number of independent trajectories is called an ensemble of $X$, and histogramming its values at any given time $t$ yields the probability distribution $p_{1}(x, t)$. If all trajectories start at $X(0)=x_{0}$, the histogram yields $p_{1 \mid 1}\left(x, t \mid x_{0}, 0\right)$. More formally, $p_{n}(A)$ denotes the unconditioned probability distribution for a number of events $A=\left\{A_{1}, \ldots, A_{n}\right\}$ to occur. Each single event $A_{i}$ has the form $\left(x_{i}, t_{i}\right)$, meaning that $X$ takes on the value $x_{i}$ at time $t_{i}$. The conditional probability distribution $p_{n \mid m}$ for the $n$ events $A_{i}(i=1, \ldots, n)$ to occur, given that $m$ events $B:=\left\{B_{j}\right\}(j=1, \ldots, m)$ occurred in the past, is defined as

$$
p_{n \mid m}(A \mid B)=\frac{p_{n+m}(A ; B)}{p_{m}(B)} .
$$

For instance, $p_{1 \mid 2}\left(x_{3}, t_{3} \mid x_{2}, t_{2} ; x_{1}, t_{1}\right)$ is the probability (distribution) that $X\left(t_{3}\right)=x_{3}$, given that first $X\left(t_{1}\right)=x_{1}$ and then $X\left(t_{2}\right)=x_{2}$. Equation 2.52 also holds true when all probabilities appearing in it are additionally conditioned on another set of even earlier events $C:=\{C\}_{k}(k \in \mathscr{N})$ :

$$
p_{n \mid m+k}(A \mid B ; C)=\frac{p_{n+m \mid k}(A ; B \mid C)}{p_{m \mid k}(B \mid C)} \quad .
$$

For readability in the following sections, we drop all subscripts on the functions $p_{n}$ and $p_{n \mid m}$, instead simply writing $p$ for all of them, even though technically speaking they are different mathematical functions. The reader is prompted to distinguish those functions by their arguments instead of by their name.

Consistency condition As a starting point for our calculations we choose the consistency condition which holds true for any probability distribution and time $t_{1}$, and is used as an axiom:

$$
\begin{aligned}
p\left(x_{2}, t_{2}\right) & =\int p\left(x_{1}, t_{1} ; x_{2}, t_{2}\right) \mathrm{d} x_{1} \\
& \stackrel{2.52}{=} \int p\left(x_{2}, t_{2} \mid x_{1}, t_{1}\right) p\left(x_{1}, t_{1}\right) \mathrm{d} x_{1} \\
\text { and } p\left(x_{2}, t_{2} \mid x_{0}, t_{0}\right) & =\int p\left(x_{2}, t_{2} ; x_{1}, t_{1} \mid x_{0}, t_{0}\right) \mathrm{d} x_{1} \\
& \stackrel{2.53}{=} \int p\left(x_{2}, t_{2} \mid x_{1}, t_{1} ; x_{0}, t_{0}\right) p\left(x_{1}, t_{1} \mid x_{0}, t_{0}\right) \mathrm{d} x_{1}
\end{aligned}
$$

As the name indicates, these statements simply formalize logical consistency: The probability to end up at some final state equals the sum of the probabilities for all paths ending there, even when conditioned on an initial state in the past.

Markov processes Throughout this thesis, we focus on so-called Markov processes. Physically speaking, this means that the current state of a system contains all information required to make best-possible predictions about its future. The history of past events does not allow for more accurate predictions. Mathematically speaking, the conditional probability fulfills the Markov condition:

$$
p\left(x_{N}, t_{N} \mid x_{N-1}, t_{N-1} ; x_{N-2}, t_{N-2} ; \ldots\right)=p\left(x_{N}, t_{N} \mid x_{N-1}, t_{N-1}\right)
$$


Chapman Kolmogorov equation Pairing the Markov condition with the consistency condition, which holds for all processes, Markovian or not, leads to the Chapman-Kolmogorov equation (CKE):

$$
\begin{aligned}
p\left(x_{2}, t_{2} \mid x_{0}, t_{0}\right) & \stackrel{2.54}{=} \int p\left(x_{2}, t_{2} \mid x_{1}, t_{1} ; x_{0}, t_{0}\right) p\left(x_{1}, t_{1} \mid x_{0}, t_{0}\right) \mathrm{d} x_{1} \\
& \stackrel{2.55}{=} \int p\left(x_{2}, t_{2} \mid x_{1}, t_{1}\right) p\left(x_{1}, t_{1} \mid x_{0}, t_{0}\right) \mathrm{d} x_{1}
\end{aligned}
$$

The CKE holds true only for Markov processes. Note that the first part of equation 2.54 looks almost identical to the CKE but holds true for any process. The difference is that the CKE considers conditional probabilities.

Master equation We now derive the master equation: the partial integro-differential equation which describes the temporal change of the probability distribution $p\left(x, t \mid x_{0}, t_{0}\right)$ in the phase space of a Markov process. Before we start, let us consider the conditional probability to be at some point $x$ at time $t+\tau$ after starting at $y$ at time $t$ :

$$
p(x, t+\tau \mid y, t)=\tau k(y, x)+\delta(x-y) \cdot\left[1-\tau \int k\left(y, x^{\prime}\right) \mathrm{d} x^{\prime}\right]+\mathscr{O}\left(\tau^{2}\right)
$$

This is a simple balance equation stating that probability flows with a rate $k(y, x)$ from $y$ to $x$ (which defines $k$ as a transition rate of probability per time, see also eq. 2.51). The second term states that the amount of probability which is still left in $y$ after a time $\tau$ equals 1 minus the total outflux. It further takes care of the proper $\tau \rightarrow 0$ behavior, as well as normalization of $p(x, t+\tau \mid y, t)$. For non-infinitesimal times $\tau$, probability may flow back from $x$ to $y$. This is a second order $\left(\propto \tau^{2}\right)$ effect, however, which is all we need to know about it. We remark that equation 2.57 can also be rigorously derived from the CKE (see e.g. chapter 4 in reference [98]). Next, we calculate the partial time derivative of the probability distribution at some point $x$ :

$$
\begin{aligned}
\partial_{t} p\left(x, t \mid x_{0}, t_{0}\right)= & \lim _{\tau \rightarrow 0} \frac{p\left(x, t+\tau \mid x_{0}, t_{0}\right)-p\left(x, t \mid x_{0}, t_{0}\right)}{\tau} \\
\stackrel{2.56}{=} \lim _{\tau \rightarrow 0} \frac{1}{\tau} \int\left[p(x, t+\tau \mid y, t) p\left(y, t \mid x_{0}, t_{0}\right)-p(x, t \mid y, t) p\left(y, t \mid x_{0}, t_{0}\right)\right] \mathrm{d} y & \stackrel{2.57}{=} \lim _{\tau \rightarrow 0} \frac{1}{\tau} \int\left[p\left(y, t \mid x_{0}, t_{0}\right) \cdot\left(\tau k(y, x)+\delta(x-y) \cdot\left[1-\tau \int k\left(y, x^{\prime}\right) \mathrm{d} x^{\prime}\right]\right)\right. \\
& \left.\quad-p\left(y, t \mid x_{0}, t_{0}\right) \cdot \delta(x-y)\right] \mathrm{d} y \\
= & \lim _{\tau \rightarrow 0} \frac{1}{\tau} \int p\left(y, t \mid x_{0}, t_{0}\right) \cdot\left(\tau k(y, x)-\delta(x-y) \cdot \tau \cdot \int k\left(y, x^{\prime}\right) \mathrm{d} x^{\prime}\right) \mathrm{d} y \\
= & \lim _{\tau \rightarrow 0} \frac{1}{\tau} \int \tau p\left(y, t \mid x_{0}, t_{0}\right) k(y, x) \mathrm{d} y-\lim _{\tau \rightarrow 0} \frac{1}{\tau}\left[\tau p\left(x, t \mid x_{0}, t_{0}\right) \int k\left(x, x^{\prime}\right) \mathrm{d} x^{\prime}\right] \mathrm{d} y \\
= & \int\left[p\left(y, t \mid x_{0}, t_{0}\right) k(y, x)-p\left(x, t \mid x_{0}, t_{0}\right) k(x, y)\right] \mathrm{d} y .
\end{aligned}
$$

This is the famous master equation. It is easily interpreted: The probability at some point $x$ changes according to the balance between total influx and outflux from all other states $y$. Note that setting the integrand above to zero corresponds to the detailed balance condition. 


\subsubsection{Kramers-Moyal expansion}

The Master-equation is a partial integro-differential equation which is often difficult to handle ${ }^{3}$. As will now be demonstrated, it is possible to convert it into an equivalent partial differential equation (PDE). To this end we change the way we denote the transition rates $k$ : Instead of having the initial and final states of the transition as arguments, the initial state and increment (step size) are chosen. Hence we define said increment $\Delta$ and the transition rate $w(x, \Delta)$ from $x$ to another state at a distance $\Delta$ from $x$ as

$$
\begin{aligned}
\Delta & :=y-x \\
\text { and } \quad w(x, \Delta)=w(x, y-x) & :=k(x, y)=k(x, \Delta+x) \quad .
\end{aligned}
$$

Inserting these definitions into the Master equation 2.58 (and dropping the condition $\left(x_{0}, t_{0}\right)$ for readability) yields

$$
\begin{aligned}
\partial_{t} p(x, t) & =\int[w(y, x-y) p(y, t)-w(x, \Delta) p(x, t)] \mathrm{d} y \\
& =\int[w(\Delta+x,-\Delta) p(\Delta+x, t)-w(x, \Delta) p(x, t)] \mathrm{d} \Delta \\
& =\int w(\Delta+x,-\Delta) p(\Delta+x, t) \mathrm{d} \Delta-p(x, t) \int w(x, \Delta) \mathrm{d} \Delta \\
& =\int w(x-\Delta, \Delta) p(x-\Delta, t) \mathrm{d} \Delta-p(x, t) \int w(x, \Delta) \mathrm{d} \Delta
\end{aligned}
$$

where in the last line, the sign of $\Delta$ was changed assuming infinite integration limits. We now expand the integrand of the first integral around $x$ and find

$$
\begin{aligned}
\partial_{t} p(x, t) & =\int \sum_{n=1}^{\infty} \frac{(-\Delta)^{n}}{n !} \frac{\partial^{n}}{\partial x^{n}}[w(x, \Delta) p(x, t)] \mathrm{d} \Delta \\
& =\sum_{n=1}^{\infty} \frac{(-1)^{n}}{n !} \frac{\partial^{n}}{\partial x^{n}}\left[p(x, t) \int \Delta^{n} w(x, \Delta) \mathrm{d} \Delta\right] \\
& =\sum_{n=1}^{\infty} \frac{(-1)^{n}}{n !} \frac{\partial^{n}}{\partial x^{n}}\left[p(x, t) \alpha^{(n)}(x)\right]
\end{aligned}
$$

where the zero-order term of the Taylor expansion cancelled out the second integral, and we have introduced the jump moments

$$
\alpha^{(n)}(x):=\int \Delta^{n} w(x, \Delta) \mathrm{d} \Delta
$$

\footnotetext{
${ }^{3}$ Due to its linearity, the Master equation is commonly solved by using characteristic functions.
} 
An alternative expression for the jump moments can be obtained as follows. For $n \in \mathbb{N}$, we define

$$
\begin{aligned}
\Psi^{(n)}(x, \tau) & :=\left.\left\langle[X(t+\tau)-X(t)]^{n}\right\rangle\right|_{X(t)=x} \\
& =\int(y-x)^{n} p(y, t+\tau \mid x, t) \mathrm{d} y \\
& \stackrel{2.57}{=} \int(y-x)^{n}\left[\tau k(y, x)+\delta(x-y) \cdot\left[1-\tau \int k\left(y, x^{\prime}\right) \mathrm{d} x^{\prime}\right]+\mathscr{O}\left(\tau^{2}\right)\right] \mathrm{d} y \\
& =\tau \int(y-x)^{n} k(y, x) \mathrm{d} y+\mathscr{O}\left(\tau^{2}\right) \\
& =\tau \int \Delta^{n} w(x, \Delta) \mathrm{d} \Delta+\mathscr{O}\left(\tau^{2}\right) \\
& =\tau \alpha^{(n)}(x)+\mathscr{O}\left(\tau^{2}\right)
\end{aligned}
$$

where, after line $3, n \geq 1$ was assumed. Hence we found

$$
\Psi^{(n)}(x, \tau)= \begin{cases}1 & \text { for } n=0 \\ \alpha^{(n)}(x) \tau+\mathscr{O}\left(\tau^{2}\right) & \text { for } n \geq 1\end{cases}
$$

and read off

$$
\begin{aligned}
\alpha^{(n)}(x) & =\lim _{\tau \rightarrow 0} \frac{\Psi_{n}(x, \tau)}{\tau} \\
& =\left.\lim _{\tau \rightarrow 0} \frac{1}{\tau}\left\langle[X(t+\tau)-X(t)]^{n}\right\rangle\right|_{X(t)=x},
\end{aligned}
$$

which then holds true only for $n \geq 1$. So far, we have assumed that $x, y$ and $\Delta$ are scalars. When they are not, the Master equation does not formally change, but its Kramers-Moyal expansion (equation 2.61) must be performed in $N$ dimensions where $\vec{x}=\left(x_{1}, \ldots, x_{N}\right)$ and $\vec{\Delta}=\left(\Delta_{1}, \ldots, \Delta_{N}\right)$. This is straightforward and will not be done here explicitly. The formerly scalar $n$-th jump moment $\alpha^{(n)}(x)$ turns into the $n$-dimensional, symmetric tensor

$$
\begin{aligned}
\alpha_{i_{1} \ldots i_{n}}^{(n)}(\vec{x}) & =\int \ldots \int \Delta_{i_{1}} \ldots \Delta_{i_{n}} w(\vec{x}, \vec{\Delta}) \mathrm{d} \Delta_{i_{1}} \ldots \mathrm{d} \Delta_{i_{n}} \\
& =\left.\lim _{\tau \rightarrow 0} \frac{1}{\tau}\left\langle\prod_{k=1}^{n} X_{i_{k}}(t+\tau)-X_{i_{k}}(t)\right\rangle\right|_{X(t)=\vec{x}}
\end{aligned}
$$

where the indices $i_{1}, \ldots, i_{n} \in\{1, \ldots, N\}$ point out one of its elements.

No approximations were made above, and hence equation 2.61 is equivalent to equation 2.58 itself. Why then use the Kramers-Moyal expansion of the Master equation? Firstly, it is a regular $\mathrm{PDE}$, while the master equation is an integro-differential equation. Secondly, one may in some cases pre-calculate the jump moments analytically and thereby simplify solving the full PDE. Thirdly, one may truncate the expansion after an appropriate number of terms and thereby simplify the problem at hand systematically.

Note that we can now identify a Markov process not only by its transition rates $k(x, y)$ (Master equation) or jump moments $\alpha^{(n)}(x)$ (Kramers-Moyal expansion), but also by statistical properties of the underlying random variable's trajectories $X(t)$ (equation 2.65). 


\subsubsection{The Fokker-Planck equation}

The theorem of Pawula [104] states that the Kramer-Moyal expansion ends after either one term, two terms, or never. We proof this theorem in appendix B. It can further be shown that non-vanishing higher-order $(k>2)$ jump moments are linked to discontinuous processes (jumpprocesses) [105]. In the $N$-dimensional physical situations which are relevant in the scope of this thesis (continuous diffusion processes), the jump moments are $\alpha^{(k)}=0$ for $k \geq 3$. This turns the Master equation into the Fokker-Planck equation:

$$
\partial_{t} p(\vec{x}, t)=-\partial_{k}\left[p(\vec{x}, t) \alpha_{k}^{(1)}(\vec{x})\right]+\frac{1}{2} \partial_{i} \partial_{j}\left[p(\vec{x}, t) \alpha_{i j}^{(2)}(\vec{x})\right]
$$

Due to Pawula's theorem, the Fokker-Planck equation is exact and not an approximation. An equivalent formulation is found via the product rule

$$
\begin{aligned}
\partial_{t} p & =-\partial_{k}\left[p \alpha_{k}^{(1)}\right]+\frac{1}{2} \partial_{i} \partial_{j}\left[\alpha_{i j}^{(2)} p\right] \\
& =-\partial_{k}\left[p \alpha_{k}^{(1)}\right]+\frac{1}{2} \partial_{i}\left[\alpha_{i j}^{(2)} \partial_{j} p\right]+\frac{1}{2} \partial_{i}\left[p \partial_{j} \alpha_{i j}^{(2)}\right] \\
& =-\partial_{k}\left[p\left(\alpha_{k}^{(1)}-\partial_{j} \alpha_{k j}^{(2)}\right)\right]+\frac{1}{2} \partial_{i}\left[\alpha_{i j}^{(2)} \partial_{j} p\right] \\
& =-\partial_{k}\left[A_{k} p\right]+\partial_{i}\left[D_{i j} \partial_{j} p\right]
\end{aligned}
$$

where we have defined the drift vector and the diffusion matrix as

$$
A_{k}(\vec{x}):=\alpha_{k}^{(1)}(\vec{x})-\partial_{j} \alpha_{k j}^{(2)}(\vec{x})
$$

and $\quad D_{i j}(\vec{x}):=\frac{1}{2} \alpha_{i j}^{(2)}(\vec{x}) \quad$.

By further denoting the probability current as

$$
\begin{aligned}
\vec{j}(\vec{x}) & :=-\vec{A} p+\mathbf{D} \vec{\nabla} p \\
& =(-\vec{A}+\mathbf{D} \cdot \vec{\nabla} \ln p) \cdot p,
\end{aligned}
$$

the Fokker-Planck equation takes on the simple form

$$
\partial_{t} p=-\vec{\nabla} \cdot((-\vec{A}+\mathbf{D} \cdot \vec{\nabla} \ln p) \cdot p)=-\vec{\nabla} \cdot \vec{j}
$$

which is a continuity equation for the total probability, unsurprisingly confirming that it is a conserved quantity. 
The physical Fokker-Planck equation We now derive the Fokker-Planck equation for an ensemble of polymer chains from a physical viewpoint by implementing (i) overdamped dynamics with hydrodynamic interactions and (ii) free energy minimization on ensemble level. The probability distribution defined on $\Omega$, the set of all chain conformations, must obey a continuity equation of the form of equation 2.71. Denoting the collection of velocities as $\vec{v}=\mathrm{d}_{t} \vec{x}$, the probability current can be written as

$$
\vec{j}=p \cdot \vec{v}
$$

As we have established in the section on hydrodynamics, $\vec{v}$ has a linear relationship with the collection of forces $\vec{F}$ acting on the chain and the diffusion matrix $\mathbf{D}$ (see page 24) translates between them:

$$
\vec{v}=T^{-1} \mathbf{D} \cdot \vec{F}
$$

where $T$ denotes the temperature. Inserting equations 2.72 and 2.73 into the continuity equation yields

$$
\partial_{t} p=-T^{-1} \vec{\nabla} \cdot(p \cdot \mathbf{D} \cdot \vec{F})
$$

At this point the overdamped dynamics and hydrodynamic interactions are already built in, but free energy minimization is not. To do so, we revisit the free energy's functional form:

$$
\begin{aligned}
F[p(\vec{x})] & =U-T S \\
& =\langle E+T \ln p\rangle \\
& =\int_{\Omega} p(\vec{x}) \cdot[E(\vec{x})+T \ln p(\vec{x})] \mathrm{d} \vec{x} .
\end{aligned}
$$

This is an integral of the term $[E(\vec{x})+T \ln p(\vec{x})]$, weighted by the probability $p(\vec{x})$. Equivalently, it can be understood as a non-weighted sum over all trajectories in the ensemble of $X$ because they are by definition distributed according to $p(\vec{x})$. We deduce that, in order to globally minimize free energy, each trajectory in the ensemble experiences a force

$$
\vec{F}=-\vec{\nabla}(E+T \ln p)
$$

and thereby contributes to global free energy minimization. That is because free energy is the physical potential of the canonical ensemble, and forces are the negative gradients of physical potentials, leading to dynamics which minimize them.

Inserting this force into equation 2.74 finally yields the physical Fokker-Planck equation:

$$
\partial_{t} p=\vec{\nabla} \cdot\left(T^{-1} p \mathbf{D} \cdot \vec{\nabla} E\right)+\vec{\nabla} \cdot(\mathbf{D} \cdot \vec{\nabla} p) \quad .
$$


While the first force term is clearly related to energy minimization, the second force term depends on the probability $p$ itself, showing that it is of a purely statistical nature. This makes sense as its origin is entropy maximization. Remarkably, this second force term also shows up in the "default" Fokker-Planck equation. This indicates that its logarithmic nature is quite naturally linked to the diffusive behavior of Markov processes in general, even without any physical considerations. To summarize, the first term (drift term) in the Fokker-Planck equation minimizes energy and the second term (diffusion term) maximizes entropy.

Now, let us study the stationary solution of the physical Fokker-Planck equation. It represents thermal equilibrium, and is found by setting the time-derivative to zero such that

$$
\vec{\nabla} \cdot \vec{j}=0
$$

Given that the hydrodynamic diffusion matrix is invertible and that the detailed balance condition (time reversal symmetry) sets $\vec{j}$ itself to zero, we find

$$
\begin{aligned}
& 0=\vec{j}=T^{-1} \mathbf{D} \cdot(-\vec{\nabla}(E+T \ln p) p) \\
& \Leftrightarrow \vec{\nabla} E \cdot p=T \vec{\nabla} \ln p \cdot p=T \vec{\nabla} p
\end{aligned}
$$

This equation can be interpreted as a local force balance between energetic and entropic forces. It is solved by the Boltzmann distribution

$$
p^{\mathrm{eq}}(\vec{x})=\frac{1}{Z} \exp (-\beta E(\vec{x})) \quad,
$$

which is not very surprising, remembering that free energy minimization is equivalent to taking on the Boltzmann distribution. What is remarkable, however, is that the Fokker-Planck perspective explicitly shows that equilibrium statistics are independent of the diffusion matrix $\mathbf{D}$, while it simply does not make an appearance in thermodynamics at all. 
Diffusion equation Let us consider the one-dimensional Fokker-Planck equation in the case $E=0$ and $D=\frac{1}{2}$. Equivalently, the jump moments are given by

$$
\begin{aligned}
\alpha^{(1)} & =0 \\
\text { and } \quad \alpha^{(2)} & =1
\end{aligned}
$$

The corresponding Fokker-Planck equation is called the diffusion equation and reads

$$
\partial_{t} p=\frac{1}{2} \partial_{x}^{2} p
$$

This equation can easily be solved analytically via Fourier transformation (see e.g. reference [106]) and the solution for $p(x, 0)=\delta(x)$ reads

$$
p(x, t \mid 0,0)=\frac{1}{\sqrt{2 \pi t}} \exp \left(-\frac{x^{2}}{2 t}\right) \quad,
$$

which has the interesting properties $\langle x\rangle=0$, but $\left\langle x^{2}\right\rangle=t$. This solution is visualized in figure 2.3.

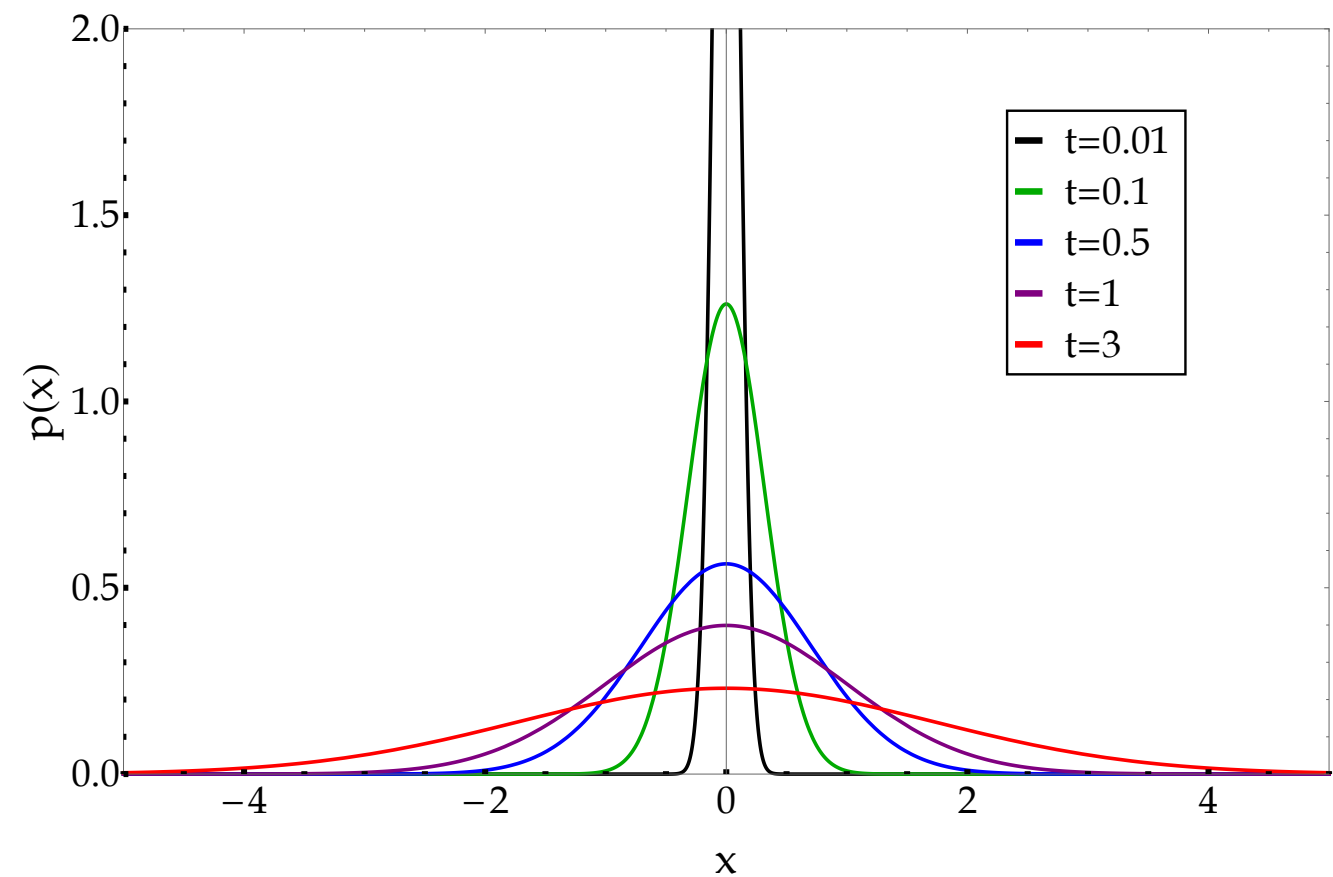

Figure 2.3: The solution of the diffusion equation plotted at different times using the initial condition $x(0)=0$. 


\subsubsection{Langevin equation}

Now we move away from the ensemble level and instead study the properties of individual trajectories $\vec{x}(t)$ within the ensemble described by the physical Fokker-Planck equation. In the previous sections, a Markov process has been uniquely characterized by its jump moments, which can be calculated from equation 2.62 on ensemble level. The same can be done on trajectory level by using the alternative expressions

$$
\alpha^{(n)}(x)=\left.\lim _{\tau \rightarrow 0} \frac{1}{\tau}\left\langle[X(t+\tau)-X(t)]^{n}\right\rangle\right|_{X(t)=x} \quad .
$$

Their evaluation requires an equation of motion for $X$ : the Langevin equation. Rather than defining it right away, let us first motivate it from a physical perspective. Between the dynamics of $\vec{x}$ (the polymer) and the dynamics of the water molecules, there is a significant separation of time scales. The configurational dynamics of the studied proteins takes place on a time scale of tens or hundreds of nanoseconds $\left(10^{-7} \mathrm{~s}-10^{-8} \mathrm{~s}\right)$, while reconfiguration times of water molecules in the fluid are on the order of tens of femtoseconds $10^{-14} \mathrm{~s}$. Therefore, even during time steps which seem very short from the protein's perspective, an enormous number of momentum transfers from the surrounding water molecules take place. Consequently, the net momentum transfer in each time step of say $10^{-11} \mathrm{~s}$ is practically stochastic from the protein's perspective and furthermore normally distributed due to the central limit theorem. To describe the dynamics of $X$, we turn back to the overdamped dynamics within a Stokes flow (see equation 2.19, page 20) and represent the discussed fluctuating momentum transfer by introducing a stochastic term $\vec{\xi}$ :

$$
\mathrm{d}_{t} \vec{x}=\vec{a}(\vec{x})+\boldsymbol{b}(\vec{x}) \cdot \vec{\xi}
$$

The coefficients $\vec{a}$ and $\boldsymbol{b}$ are to be determined later on to match the Fokker-Planck equation's jump moments. The stochastic term $\vec{\xi}$ has a mean value of zero, as any non-zero mean could be subtracted from $\vec{\xi}$ and be absorbed into $\vec{a}$. Each vector component of $\vec{\xi}$ independently takes on values which we assume to be uncorrelated in time - a convenient idealization justified by the separation of time scales. Therefore

$$
\begin{aligned}
\langle\vec{\xi}(t)\rangle & =\overrightarrow{0} \\
\text { and }\left\langle\xi_{i}(0) \xi_{j}(t)\right\rangle & =\left\{\begin{array}{ll}
\delta_{i j} f(t) & \text { if } t=0 \\
0 & \text { if } t \neq 0
\end{array} \quad \text { where } i, j \in\{1,2,3\}\right.
\end{aligned}
$$

The function $f(t)$ must be a Dirac delta function for the following reason. The Wiener-Khinchin theorem states that for any time series $\xi(t)$, the integral over its autocorrelation function $\left\langle\xi_{j}(0) \xi_{j}(t)\right\rangle$ is related to its power spectrum $\left|\hat{\xi}_{j}\right|^{2}$ via

$$
|\hat{\boldsymbol{\xi}}(s)|^{2}=\int_{-\infty}^{\infty}\left\langle\xi_{j}(0) \xi_{j}(t)\right\rangle \exp (-i 2 \pi s t) \mathrm{d} t
$$


Therefore, if this integral is zero, the power spectrum is zero, and then $\xi$ itself is zero. The integral must thus be finite and since the autocorrelation function is zero for $t_{1} \neq t_{2}$, it must be a delta function. It is thus an artifact of the idealization that $\vec{\xi}$ is uncorrelated at different times that its autocorrelation is infinite - otherwise it would not have a net effect on the trajectory. Consequently, its power spectrum is a constant, which earns $\xi$ the title of "white noise". Due to its stochastic nature, the Langevin is a stochastic differential equation, and we write it in differential form

$$
\mathrm{d} \vec{x}=\vec{a} \mathrm{~d} t+\boldsymbol{b} \cdot \vec{\xi} \mathrm{d} t
$$

such that, formally, we have

$$
\vec{x}(t)=\vec{x}(0)+\int_{0}^{t} \vec{a}\left(\vec{x}\left(t^{\prime}\right)\right) \mathrm{d} t^{\prime}+\int_{0}^{t} \boldsymbol{b}\left(\vec{x}\left(t^{\prime}\right)\right) \cdot \vec{\xi}\left(t^{\prime}\right) \mathrm{d} t
$$

The first integral is a regular, deterministic integral as known from standard calculus. The second integral, however, is a stochastic integral whose practical evaluation must be discussed.

The Wiener process Defining each component of the integral of $\vec{\xi}(t)$ as

$$
\Delta W(t)=W(t+\Delta t)-W(t)=\int_{t}^{t+\Delta t} \xi \mathrm{d} t
$$

such that $\mathrm{d} \vec{W}=\vec{\xi} \mathrm{d} t$, one finds

$$
\begin{aligned}
\langle\Delta W\rangle & =\int_{t}^{t+\Delta t}\langle\xi\rangle \mathrm{d} t=0 \\
\left\langle\Delta W^{2}\right\rangle & =\int_{t}^{t+\Delta t} \int_{t}^{t+\Delta t}\left\langle\xi(s) \cdot \xi\left(s^{\prime}\right)\right\rangle \mathrm{d} s^{\prime} \mathrm{d} s \\
& =\int_{t}^{t+\Delta t} \int_{t}^{t+\Delta t} \delta\left(s-s^{\prime}\right) \mathrm{d} s^{\prime} \mathrm{d} s \\
& =\Delta t
\end{aligned}
$$

The stochastic process $W(t)$ is called the Wiener process. A typical size of its time increments is

$$
\sqrt{\left\langle\Delta W^{2}\right\rangle}=\sqrt{\Delta t} .
$$

Assuming that the Wiener increments are Gaussian in nature (due to the central limit theorem, the net momentum transfer due to the stochastic force $\xi$ must be Gaussian as a result of a large number of collisions with water molecules), their probability distribution is determined by their first two moments (being 0 and $\Delta t$ respectively) and reads

$$
p(W(t+\Delta t), t+\Delta t \mid W(t), t)=\frac{1}{\sqrt{2 \pi \Delta t}} \exp \left(-\frac{\Delta W^{2}}{2 \Delta t}\right) \quad .
$$


Note that this is precisely the solution of the diffusion equation (see equation 2.83). In fact, the Wiener process has the same jump moments

$$
\begin{aligned}
\alpha^{(1)} & =0 \\
\text { and } \quad \alpha^{(2)} & =1 \quad .
\end{aligned}
$$

as the diffusion equation does (see paragraph "Jump moments for the Langevin equation" below) and can therefore be regarded as its analogue at the level of trajectory - the histogram over an ensemble of Wiener processes equals the solution of the diffusion equation!

The Wiener process is stationary (increments do not depend on $t$ ) and spatially homogeneous (increments do not depend on $W$ ). It is also Markovian since increments at different times are independent of each other.

Trajectories of the Wiener process Trajectories $\left\{W_{0}, \ldots, W_{N}\right\}=\{W(0), W(\Delta t), \ldots, W(N \Delta t)\}$ of the Wiener process can be generated by successively drawing increments $W_{i+1}-W_{i}$ from the Gaussian probability distribution 2.93. This is done as follows: Each increment is first drawn from a standard normal distribution (zero mean and variance one), and then multiplied with $\sqrt{\Delta t}$ such that its variance equals $\Delta t$. A number of such trajectories are displayed in figure 2.4. It is therefore intuitively clear that the sample paths are continuous because

$$
\lim _{\Delta t \rightarrow 0} \Delta W \propto \lim _{\Delta t \rightarrow 0} \sqrt{\Delta t} \rightarrow 0
$$

but at the same time nowhere differentiable because

$$
\lim _{\Delta t \rightarrow 0} \frac{\Delta W}{\Delta t} \propto \lim _{\Delta t \rightarrow 0} \frac{1}{\sqrt{\Delta t}} \rightarrow \infty
$$

Mathematically more rigorous proofs of the continuity and non-differentiability of the Wiener process (which require technically more precise notions of limits of stochastic variables) are available in literature $[1,107]$. The non-differentiability in particular implies that, technically speaking, the process $\xi$ with the properties given by equation 2.86 does not exist. Its integral, the Wiener process, does exist however, and thus the Langevin equation has meaningful solutions.

The same properties (equations 2.95 and 2.96) hold true for the trajectories of the Langevin equation 2.88 because the Wiener increments are simply multiplied with the matrix $\mathbf{b}$ and then the vector $\vec{a} \cdot \Delta t$ is added. It turns out, however, that the statistical properties (jump moments) of the resulting process depend on where in the interval $[t, t+\Delta t]$ the matrix $\boldsymbol{b}$ is evaluated, no matter how small $\Delta t$ is. This curiosity is being discussed below under the paragraph "Itô versus Stratonovich". 


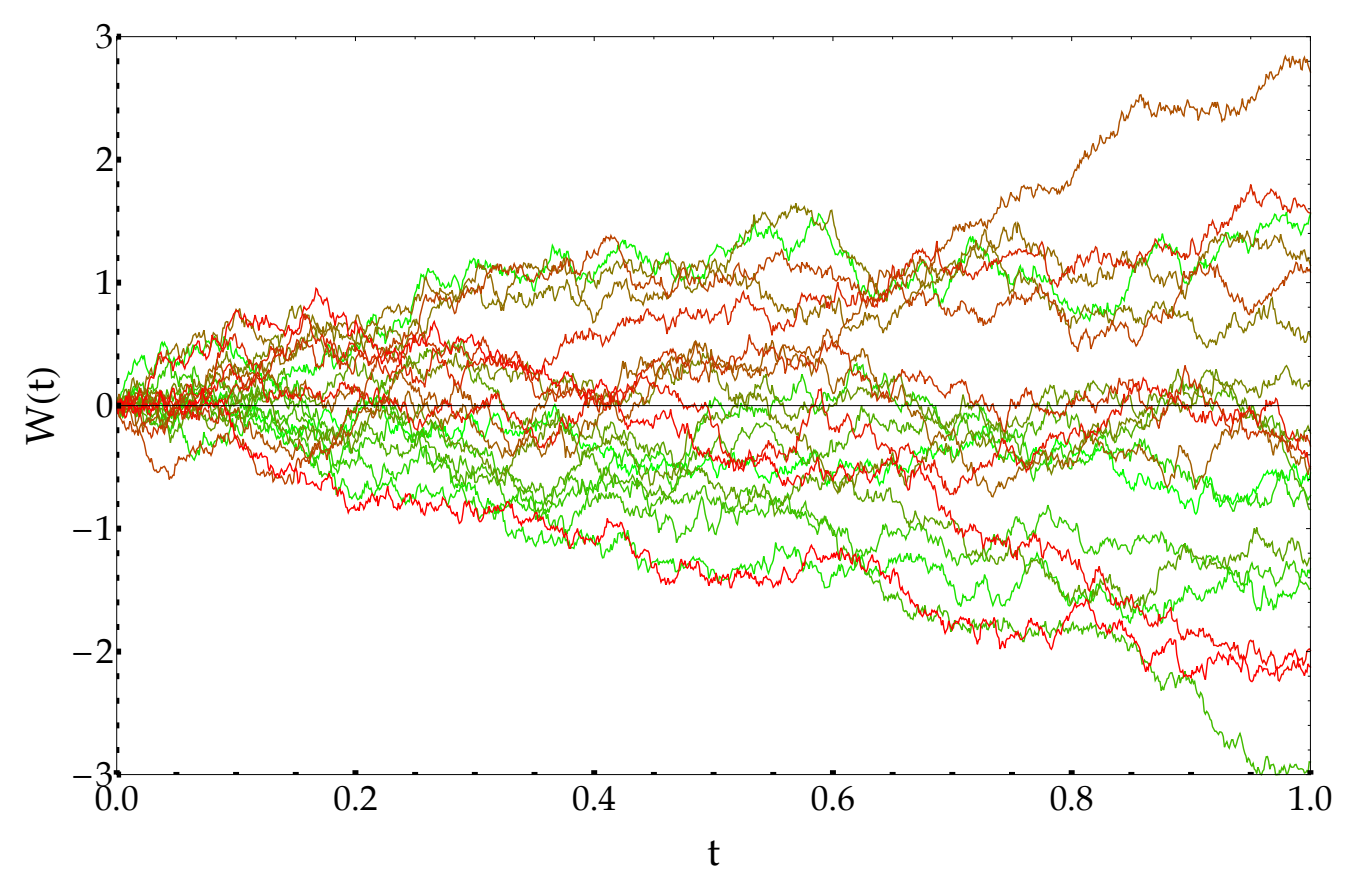

Figure 2.4: Twenty independent trajectories of the Wiener process, all starting at zero. They are everywhere continuous, but nowhere differentiable. Viewed as an ensemble, their distribution spreads over time according to the diffusion equation 2.82. Therefore, this distribution is at all times $t$ a Gaussian with zero mean and variance $t$, see also figure 2.3.

Another quite fascinating property of the Wiener process is that the time resolution used to arrive at a final increment is irrelevant - starting at some value $W(t)$, drawing a single increment $\Delta W$ at time $t+\Delta t$ from a Gaussian (statistically) gives the same result as drawing $i=1, \ldots, N$ successive (smaller) increments $\Delta W_{i}$ with time resolution $\Delta t / N$ such that $\Delta W=\sum_{i} \Delta W_{i}$. That is because

$$
\begin{aligned}
\langle\Delta W\rangle & =\sum_{i}\left\langle\Delta W_{i}\right\rangle=0 \\
\text { and } \quad\left\langle\Delta W^{2}\right\rangle & =\sum_{i j}\left\langle\Delta W_{i} \Delta W_{j}\right\rangle=\sum_{i}\left\langle\Delta W_{i}^{2}\right\rangle=N \frac{\Delta t}{N}=\Delta t
\end{aligned}
$$

This is consistent with the fact that the final increment could have just been drawn from its own Gaussian probability distribution in the first place. Intuitively speaking, this means that the trajectories in figure 2.4 would (statistically) look the same if the time resolution during their generation was increased. The practical limitation of numerical time steps in a Langevin simulation hence stems solely from the fact that the coefficients $\vec{a}$ and $\mathbf{b}$ are in general not constant. 
The jump moments for the Langevin equation In order to calculate the jump moments

$$
\alpha^{(n)}(x)=\left.\lim _{\tau \rightarrow 0} \frac{1}{\tau}\left\langle[X(t+\tau)-X(t)]^{n}\right\rangle\right|_{X(t)=x}
$$

of the Langevin equation $\mathrm{d}_{t} \vec{x}=\vec{a}+\mathbf{b} \cdot \vec{\xi}$, we prepare the term

$$
\begin{aligned}
& =\left[x_{i}(t+\tau)-x_{i}(t)\right]^{n} \\
& =\left[\int_{t}^{t+\tau} \mathrm{d}_{t} x_{i}\left(t^{\prime}\right) \mathrm{d} t^{\prime}\right]^{n} \\
& =\left[\int_{t}^{t+\tau}\left[a_{i}\left(x\left(t^{\prime}\right)\right)+b_{i j}\left(x\left(t^{\prime}\right)\right) \xi_{j}\left(t^{\prime}\right)\right] \mathrm{d} t^{\prime}\right]^{n} \\
& =\left[\int _ { t } ^ { t + \tau } \left[a_{i}(\vec{x}(t))+\partial_{k} a_{i}(\vec{x}(t)) \cdot\left(x_{k}\left(t^{\prime}\right)-x_{k}(t)\right)+\ldots\right.\right. \\
& \left.\left.\quad+b_{i j}(\vec{x}(t)) \cdot \xi_{j}(t)+\partial_{k} b_{i j}(\vec{x}(t)) \cdot \xi_{j}\left(t^{\prime}\right) \cdot\left(x_{k}\left(t^{\prime}\right)-x_{k}(t)\right)+\ldots\right] \mathrm{d} t^{\prime}\right]^{n}
\end{aligned}
$$

where we have Taylor expanded the integrand to take into account that it changes during the time interval $[t, t+\tau]$. Here, we have only performed the first step in an iterative process. Next we would need to reinsert $x_{k}\left(t^{\prime}\right)-x_{k}(t)$ into itself because it occurs in the first line as well as in the Taylor expanded integrands. The reinserted terms then involve that same integral again and again. Making use of $\langle\vec{\xi}\rangle=\overrightarrow{0},\left\langle\xi(t) \xi\left(t^{\prime}\right)\right\rangle=\delta\left(t-t^{\prime}\right)$ and realizing that the jump moments are only interested in terms proportional to $\tau$, this iterative process can quickly be truncated.

For $n=1$, one finds

$$
\begin{aligned}
\alpha_{i}^{(1)}(\vec{y} \mid \text { Strato }) & =\left.\lim _{\tau \rightarrow 0} \frac{1}{\tau}\left\langle\left[x_{i}(t+\tau)-x_{i}(t)\right]\right\rangle\right|_{x(t)=y} \\
& =a_{i}(\vec{y})+b_{j k} \partial_{j} b_{i k}(\vec{y})
\end{aligned}
$$

where, interestingly, a term from the first reinsertion-iteration has survived the limiting process. For $n=2$ one finds

$$
\begin{aligned}
\alpha_{i j}^{(2)}(\vec{y} \mid \text { Strato }) & =\left.\lim _{\tau \rightarrow 0} \frac{1}{\tau}\left\langle\left[x_{i}(t+\tau)-x_{i}(t)\right]^{2}\right\rangle\right|_{x(t)=y} \\
& =b_{i k} b_{j k}(\vec{y})
\end{aligned}
$$

All higher order jump moments are zero, because for $n \geq 3$, no terms remain which are of order $\tau$. The reason why we supplemented our notation with the term "Strato" will now be discussed. 
Itô versus Stratonovich interpretation Carefully studying the calculations of the first jump moment $\alpha_{i}^{(1)}$ above, it turns out that the additional term $b_{j k} \partial_{j} b_{i k}(\vec{y})$ comes from the Taylor expansion of the integrand in equation 2.99. This Taylor expansion takes into account that the integrand is not constant during the time interval $[t, t+\tau]$ over which it is integrated. In regular calculus, this would not need to be taken into account due to the limit $\tau \rightarrow 0$ which is applied to the integral: no Taylor-expanded terms related to the deterministic force $\vec{a}$ (i.e. regular calculus) remain after taking this limit. It is solely a result of the properties of the stochastic force that a Taylor-expanded term contributes to the first jump moment: Because $(\xi \mathrm{d} t)^{2}=\mathrm{d} W^{2} \propto \mathrm{d} t$, a term which would be of second order in regular calculus scales linearly with $\tau$, and thus does not vanish. In other words: It matters at which time during $[t, t+\mathrm{d} t]$ the matrix $\boldsymbol{b}(\vec{x}(t))$ is evaluated, no matter how small $\mathrm{d} t$ is. In the calculations above, we have effectively evaluated $\boldsymbol{b}(\vec{x}(t))$ at $\vec{x}(t+\tau / 2)$. This is a choice we have made, which is known as the Stratonovich interpretation: the prefactor of the noise term is evaluated in the middle of the infinitesimal time step. An alternative choice is the Itô interpretation, which evaluates the prefactor of the noise term at the beginning of the time step. Within the Itô interpretation, the integrand in equation 2.99 therefore does not need to be Taylor expanded, which simplifies the calculation. The resulting jump moments read

$$
\begin{aligned}
\alpha_{i}^{(1)}(\vec{y} \mid \mathrm{It} \hat{\mathrm{o}}) & =a_{i}(\vec{y}) \\
\text { and } \quad \alpha_{i j}^{(2)}(\vec{y} \mid \mathrm{It} \hat{)}) & =b_{i k} b_{j k}(\vec{y}) \quad .
\end{aligned}
$$

The consequence of the differing jump moments is that the Langevin equation on its own does not have well-defined statistical properties. In fact, the Langevin equation is meaningless until it is supplemented by an interpretation rule such as "Itô" or "Stratonovich". It must be noted, however, that any Itô-Langevin equation can easily be transformed into an equivalent StratonovichLangevin equation (and vice versa) by simply compensating the additional term in the first jump moment by redefining $a_{i} \mapsto a_{i}-b_{j k} \partial_{j} b_{i k}(\vec{y})$. After this transformation, both interpretations yield identical jump moments and thus describe the same Markov process. Note that the Itô and Stratonovich interpretations automatically coincide if the matrix $\mathbf{b}$ does not depend on the system's state $\vec{x}$.

We remark that there are other interpretation rules such as the Hänggi-Klimontovich interpretation, which evaluates the noise term at the end of the infinitesimal time interval [108]. Which interpretation to use is a question of modeling, and for further reading, I highly recommend Van Kampen's paper "Itô versus Stratonovich" [109] which is clear and short. For this thesis, however, this question is irrelevant because we do not model the system under consideration in terms of the Langevin equation at all. All the physics has already been implemented within the Fokker-Planck equation 2.77 which determines the physically correct jump moments. Thus we can make use of this Fokker-Planck equation and find the equivalent Itô-Langevin equation, solely owing to the fact that the Itô-interpretation leads to simpler numerics. 
Translation between Langevin and Fokker-Planck equation For better readability, let us repeat the Fokker-Planck equation in terms of jump moments (equation 2.67):

$$
\partial_{t} p=-\partial_{i}\left[p \alpha_{i}^{(1)}\right]+\frac{1}{2} \partial_{i} \partial_{j}\left[p \alpha_{i j}^{(2)}\right] \quad,
$$

and directly compare it with the physical Fokker-Planck equation (equation 2.77):

$$
\begin{aligned}
\partial_{t} p & =T^{-1} \partial_{i}\left[p D_{i j} \partial_{j} E\right]+\partial_{i}\left(D_{i j} \partial_{j} p\right) \\
& =T^{-1} \partial_{i}\left[p D_{i j} \partial_{j} E\right]+\partial_{i} \partial_{j}\left[D_{i j} p\right]-\partial_{i}\left[\partial_{j} D_{i j} \cdot p\right] \\
& =\partial_{i}\left[\left(T^{-1} D_{i j} \partial_{j} E-\partial_{j} D_{i j}\right) \cdot p\right]+\partial_{i} \partial_{j}\left[D_{i j} p\right] .
\end{aligned}
$$

The physical jump moments can therefore be read off to be

$$
\begin{aligned}
\alpha_{i}^{(1)} & =-T^{-1} D_{i j} \partial_{j} E+T \partial_{j} D_{i j} \\
\text { and } \quad \alpha_{i j}^{(2)} & =2 D_{i j} .
\end{aligned}
$$

The following Itô-Langevin equation has the same jump moments:

$$
\mathrm{d} \vec{r}=T^{-1} \mathbf{D} \cdot[-\vec{\nabla} E] \mathrm{d} t+\left(\vec{\nabla}^{T} \cdot \mathbf{D}\right)^{T} \mathrm{~d} t+\sqrt{2} \mathbf{B} \cdot \mathrm{d} \vec{W}
$$

where $\mathbf{B}$ is the Cholesky decomposition of $\mathbf{D}$, meaning that $\mathbf{D}=\mathbf{B} \cdot \mathbf{B}^{T}$. Due to the equivalence to the Fokker-Planck equation, the dynamics described by this Langevin equation samples the Boltzmann distribution $p \propto \exp (-E / T)$ independently of the matrices $\boldsymbol{D}$ and $\boldsymbol{B}$. The diffusion matrix $\mathbf{D}$ is a function of the coordinates $\vec{r}$ itself, and it should be noted that the diffusion matrix in an infinite medium (see equation 2.29, page 24) is divergence-free, while the diffusion matrix near a wall (see equation 2.31, page 25) is not.

The last term is stochastic, and it is referred to as thermal noise or Brownian noise. It can alternatively be understood as a force $\sqrt{2} T\left(\mathbf{B}^{T}\right)^{-1} \mathrm{~d} \vec{W} / \mathrm{d} t$ which would then be added to the parenthesis in the first term. The equation $\mathbf{D}=\mathbf{B} \cdot \mathbf{B}^{T}$ is called a fluctuation-dissipation relation because it links the noise strength $\mathbf{B}$ to the friction matrix $\Gamma=T \mathbf{D}^{-1}$. The fact that the two are linked is remarkable, and heuristically owes to the fact that both solvent friction as well as thermal fluctuation are caused by the same fluid.

Considering the Langevin equation for an assembly of $N$ objects in three-dimensional space (e.g. a polymer chain), we saw in section 2.1.5 that the diffusion matrix $\mathbf{D}$ consists of $N^{2} 3 \times 3$-blocks $\mathbf{D}_{i j}$. With $\vec{r}=\left(\vec{r}_{1}^{T} \ldots \vec{r}_{N}^{T}\right)^{T}$ being the collection of Cartesian coordinates, the Langevin equation can be written as

$$
\mathrm{d} \vec{r}_{i}=T^{-1} \sum_{j=1}^{N} \mathbf{D}_{i j} \cdot\left[-\vec{\nabla}_{j} E\right] \mathrm{d} t+\left(\vec{\nabla}^{T} \cdot \mathbf{D}\right)_{i} \mathrm{~d} t+\sqrt{2} \sum_{j=1}^{N} \mathbf{B}_{i j} \cdot \mathrm{d} \vec{W}_{j}
$$

To summarize, the following Fokker-Planck equation and Itô-Langevin equation are equivalent:

$$
\begin{aligned}
\partial_{t} p & =-\partial_{i}\left[a_{i} p\right]+\frac{1}{2} \partial_{i} \partial_{j}\left[b_{i k} b_{j k} p\right] \\
\mathrm{d} \vec{x} & =\vec{a} \mathrm{~d} t+\boldsymbol{b} \cdot \mathrm{d} \vec{W}
\end{aligned}
$$


This equivalence must be understood as before: The Fokker-Planck equation describes how an entire ensemble of trajectories evolves. The Langevin equation describes a single trajectory within that ensemble. Since all trajectories in the ensemble are identical, in practice, solutions to the Langevin equation sample the Boltzmann distribution over time.

Remember also that the physical Fokker-Planck equation describes ensemble dynamics which minimizes its free energy. The free energy has an energetic part and an entropic part. Revisiting the calculations above from this perspective, one finds that the first term in the Langevin equation 2.106 minimizes energy, and that the second and third (random) term maximize entropy.

\subsubsection{Constrained Langevin equation}

In the following, we present aspects of the theory of constrained Brownian motion which can also be found in reference [110]. Many polymer models, including ours, are subject to geometric constraints. Let us denote them as

$$
g_{\mu}(\vec{x})=0 \quad \text { where } \quad \mu=1, \ldots, M \quad .
$$

For example, we are going to study polymer chains whose bond lengths are fixed (inextensible chains, see figure 2.6). The Langevin dynamics above, however, is unconstrained and must therefore be modified in order to take the constraints into account. This can be achieved in two ways, both of which require additional physical forces acting on the polymer chain.

Firstly (see green line in figure 2.5), one can simply punish deviations from the constraints by introducing an additional energy term. The (negative) gradient of this energy term describes restoring forces which push the system back towards the constraints. In the presence of thermal noise, the elastic constant of these forces must be chosen larger the more accurately the constraints are to be fulfilled. This limits the allowed numerical time step severely. Moreover, the constraints need to be violated in the first place in order for the restoring forces to react. Since the Langevin equation remains form-invariant, the dynamics of this first method will still sample the correct Boltzmann distribution for the modified energy function.

Secondly (see red dot in figure 2.5), it is possible to exactly preserve the constraints in each time step by introducing constraining forces which include Lagrangian multipliers (like in the Lagrange I formalism the reader might know from classical mechanics). The constraining forces enter the Langevin equation 2.107 by modifying the force term as follows:

$$
-\vec{\nabla}_{j} E \mapsto-\vec{\nabla}_{j} E+\sum_{\mu=1}^{M} \lambda_{\mu} \vec{\nabla}_{j} g_{\mu}(\vec{x})
$$

Here, $\lambda_{\mu}$ is the Lagrangian multiplier associated with the $\mu$-th constraint. When solving the Langevin equation, the Lagrangian multipliers must be determined from the constraints in each time step anew. Using this method, the constraints are fulfilled exactly. 


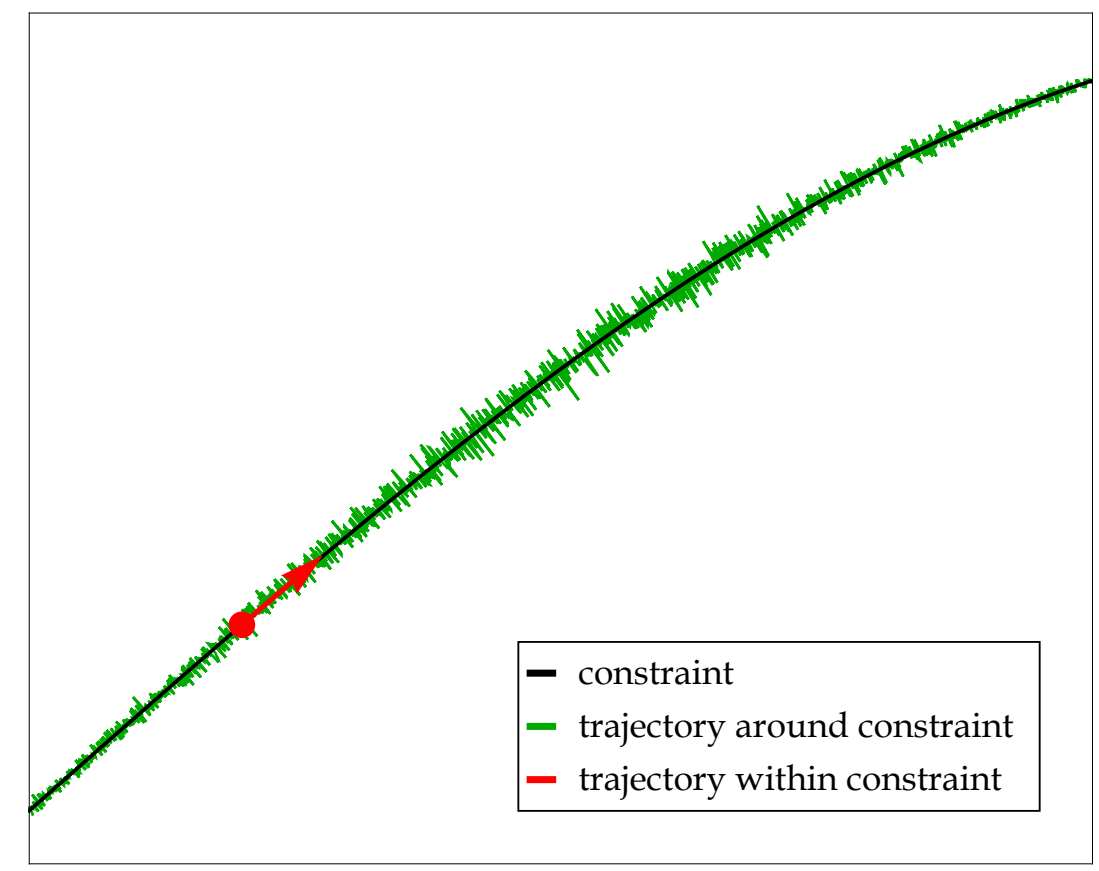

Figure 2.5: A two-dimensional sketch of $\Omega=\mathbb{R}^{3 N}$. The black curve represents the set of all chain conformations which fulfill the geometric constraints imposed on the dynamics. The green trajectory is subject to strong restoring forces which push it back towards the constraint, and thus it only approximately fulfills them. It correctly samples the Boltzmann distribution because it can be generated from the default form of the Langevin equation. The red dot, on the other hand, undergoes dynamics within the constraint which is described by a modified Langevin equation including Lagrangian multipliers. The (red) dynamics within the constraint disregard the entropy of the fluctuations (width of green fluctuations) and therefore do not correctly minimize free energy. This error is easily remedied by adding a force term which is the gradient of this entropy. This extra force pushes the red dot along the constraint towards regions of higher entropy (wider green fluctuations) as indicated by the red arrow.

The Langevin equation is not form-invariant, however, and therefore (for the time being) it is unclear whether the resulting dynamics still correctly samples the Boltzmann distribution and minimizes free energy.

We choose the second method of using Lagrangian multipliers in this thesis as it fulfills the constraints exactly while not entailing additional model parameters. It turns out that the modified Langevin equation does not minimize free energy. This is because it (by construction) does not take into account the entropy contribution $S_{C}$ of fluctuations around the constraints which the limiting case of the first method (for infinitely strong restoring forces) does while having correct statistics. Since fluctuations along and around the constraints can take on values independently of each other, their entropies are additive due to entropy's logarithmic dependence on $p$. 
Fortunately, the entropy $S_{C}$ can be calculated analytically and thus the constrained Lagrangian dynamics can be repaired by adding its gradient (with respect to $\vec{x}$ ) as an additional force:

$$
-\vec{\nabla}_{i} E \mapsto-\vec{\nabla}_{i} E+\sum_{\mu=1}^{M} \lambda_{\mu} \vec{\nabla}_{i} g_{\mu}(\vec{x})+T \vec{\nabla}_{i} S_{C}
$$

Thereby, the full free energy $F=\langle E\rangle-T S$ (not only $\langle E\rangle-T \cdot\left(S-S_{C}\right)$ ) is minimized. The entropy $S_{C}$ is explicitly calculated for general geometric constraints in appendix C. Its gradient is often called the (entropic) pseudoforce and reads

$$
\vec{\nabla}_{i} S_{C}=-\frac{1}{2} \vec{\nabla}_{i} \ln \operatorname{det} \mathbf{G}
$$

where the matrix elements of $\mathbf{G}$ are

$$
G_{k j}=\vec{\nabla}_{i} g_{k} \cdot \vec{\nabla}_{i} g_{j}
$$

The constrained Langevin equation which correctly minimizes free energy then reads

$$
\begin{aligned}
\mathrm{d} \vec{r}_{i}= & T^{-1} \sum_{j=1}^{N} \mathbf{D}_{i j} \cdot\left[-\vec{\nabla}_{j}\left(E-T S_{C}\right)+\sum_{\mu=1}^{M} \lambda_{\mu} \vec{\nabla}_{j} g_{\mu}\right] \mathrm{d} t \\
& +\left(\vec{\nabla}^{T} \cdot \mathbf{D}\right)_{i} \mathrm{~d} t+\sqrt{2} \sum_{j=1}^{N} \mathbf{B}_{i j} \cdot \mathrm{d} \vec{W}_{j}
\end{aligned}
$$

It must be supplemented with the constraints to determine the values of the Lagrangian multipliers $\lambda_{\mu}$ and with the interpretation rule "Itô", the numerical method for which is explained in section 4.2 on page 87 .

Inextensibility and bound-end constraints There are two types of constraints which are relevant in the scope of this thesis. In both cases, the abstract microstate $\vec{x}$ denotes the $i=1, \ldots, N$ Cartesian coordinates $\vec{r}_{i}$ of a discrete chain's vertices. Firstly, one end of some simulated chains is bound to a surface which will be referred to as the bound-end constraint:

$$
g_{0}(\vec{r})=\left|\vec{r}_{1}\right|-\text { const. }=0
$$

The other constraint is that all simulated chains are inextensible. This means that the bond lengths between their adjacent beads are fixed:

$$
g_{\mu}(\vec{r})=\left|\vec{r}_{\mu}-\vec{r}_{\mu-1}\right|-\text { const. }=0
$$

Both type of constraints are visualized in figure 2.6. We started with index zero to emphasize that the bound-end constraint is "optional" in the applications later on, while the $\mu=1, \ldots, N-1$ inextensibility constraints are not. 


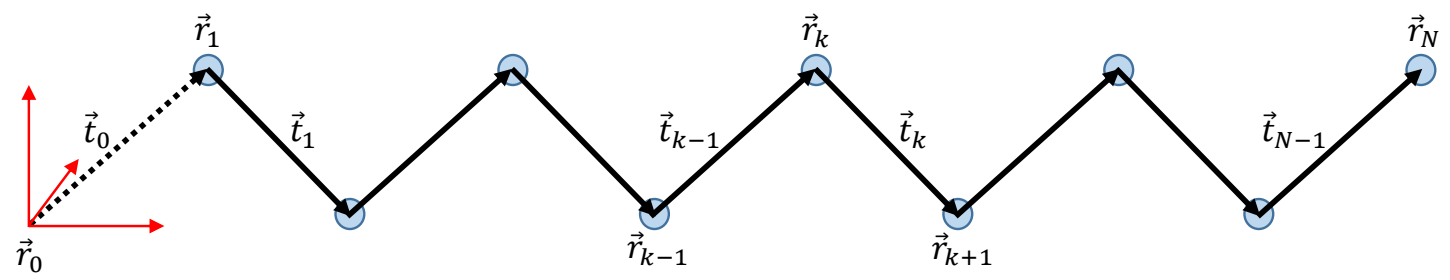

Figure 2.6: A sketch of a model polymer consisting of $N$ beads (vertices). The black lines' (rods) fixed lengths represent the inextensibility constraint (non-dashed), and the bound-end constraint (dashed). Each rod exerts a force on its adjacent beads such that its own length is preserved.

From a physical perspective, one may think of each constraint as being imposed by a rod of fixed length. Each of the $\mu=0, \ldots, N-1$ rods acts with a constraint force on the beads connected to it. The constraint forces have magnitudes $\lambda_{\mu}$ (Lagrangian multipliers) and must be directed parallel to the rod's orientation which are given by the chain's tangent vectors

$$
\vec{t}_{\mu}=\frac{\vec{r}_{\mu+1}-\vec{r}_{\mu}}{\left|\vec{r}_{\mu+1}-\vec{r}_{\mu}\right|}
$$

where $\vec{r}^{(0)}$ is not a variable but a fixed point in space that we choose to be the origin of our coordinate system such that

$$
\vec{t}_{0}=\frac{\vec{r}_{1}}{\left|\vec{r}_{1}\right|}
$$

Note that these vectors (directions of constraint forces) coincide with the gradients of the constraints. The constraint force $\lambda_{\mu} \vec{t}_{\mu}$ then acts on its two adjacent beads with opposite sign such that no total force is generated on the chain (except for the bound-end constraint, which exerts a force on e.g. a surface). Thus, the total force (including thermal noise) on bead $i$ is modified as

$$
\vec{F}_{i}=\vec{F}_{i}^{\mathrm{uc}}+\lambda_{i} \vec{t}_{i}-\lambda_{i-1} \vec{t}_{i-1}
$$

where we set $\lambda_{N}=0$ for a simpler notation and the total sum of unconstrained forces (including thermal noise) acting on the beads is formally given by

$$
\vec{F}^{\mathrm{uc}}=-\vec{\nabla}\left(E-T S_{C}\right)+\mathbf{D}^{-1} \cdot\left(\vec{\nabla}^{T} \cdot \mathbf{D}\right)+\sqrt{2}\left(\mathbf{B}^{T}\right)^{-1} \mathrm{~d} \vec{W} / \mathrm{d} t \quad .
$$

The sign convention used here implies that if $\lambda_{\mu}>0$, the corresponding rod counteracts being pulled apart by unconstrained forces on beads $\mu+1$ and $\mu$. Putting everything together, the Langevin equation constrained by bound-end and inextensibility constraints reads

$$
\mathrm{d} \vec{r}_{i}=T^{-1} \sum_{j=1}^{N} \mathbf{D}_{i j} \cdot\left[-\vec{\nabla}_{j}\left(E-T S_{C}\right)+\lambda_{i+1} \vec{t}_{i+1}-\lambda_{i} \vec{t}_{i}\right] \mathrm{d} t+\sqrt{2} \sum_{j=1}^{N} \mathbf{B}_{i j} \cdot \mathrm{d} \vec{W}_{j}
$$

where the gradient of the constraint entropy can be found in appendix C. Furthermore, $\lambda_{N}=0$, and in order to drop the bound-end constraint, one only needs to set $\lambda_{0}$ to zero as well. 


\subsubsection{Diffusion coefficient and hydrodynamic radius}

Let us study a 3 -dimensional object with a constant $3 \times 3$ diffusion matrix $\mathbf{D}=\mathbf{B} \cdot \mathbf{B}^{T}$ in absence of a potential energy function, i.e. $E=0$. With equation 2.106 on page 47 , we established that its dynamics is described by the following Langevin equation:

$$
\mathrm{d} \vec{r}=\sqrt{2} \mathbf{B} \cdot \mathrm{d} \vec{W}
$$

Multiplying this equation with its own transpose from the right, it follows that

$$
\mathrm{d} \vec{r} \cdot \mathrm{d} \vec{r}^{T}=2 \mathbf{B} \cdot \mathrm{d} \vec{W} \cdot \mathrm{d} \vec{W}^{T} \cdot \mathbf{B}^{T}
$$

Performing an ensemble average $\langle\cdot\rangle$ on both sides leads to

$$
\begin{aligned}
\left\langle\mathrm{d} \vec{r} \cdot \mathrm{d} \vec{r}^{T}\right\rangle & =2 \mathbf{B} \cdot\left\langle\mathrm{d} \vec{W} \cdot \mathrm{d} \vec{W}^{T}\right\rangle \cdot \mathbf{B}^{T} \\
& =2 \mathbf{B} \cdot \mathbb{1}_{3 \times 3} \cdot \mathbf{B}^{T} \mathrm{~d} t \\
& =2 \mathbf{D} \mathrm{d} t .
\end{aligned}
$$

Taking the trace of both sides yields

$$
\left\langle\mathrm{d} r^{2}\right\rangle=2 \operatorname{Tr} \mathbf{D} \mathrm{d} t
$$

This equation holds for infinitesimal time increments. The long term behavior can be found from the sum of a large number of such infinitesimal increments. Indexing them as $\mathrm{d} \vec{r}_{i}$ such that $\vec{r}=\sum_{i} \mathrm{~d} \vec{r}_{i}$ and $t=\sum_{i} \mathrm{~d} t$, and noting that they are statistically independent shows that

$$
\begin{aligned}
\left\langle r^{2}\right\rangle & =\left\langle\vec{r}^{T} \cdot \vec{r}\right\rangle \\
& =\left\langle\left(\sum_{i} \mathrm{~d} \vec{r}_{i}\right)^{T} \cdot\left(\sum_{j} \mathrm{~d} \vec{r}_{j}\right)\right\rangle \\
& =\sum_{i} \sum_{j}\left\langle\mathrm{~d} \vec{r}_{i}^{T} \cdot \mathrm{d} \vec{r}_{j}\right\rangle \\
& =\sum_{i} \sum_{j} \delta_{i j} 2 \operatorname{Tr} \mathbf{D} \mathrm{d} t \\
& =\sum_{i} 2 \operatorname{Tr} \mathbf{D} \mathrm{d} t \\
& =2 \operatorname{Tr} \mathbf{D} t .
\end{aligned}
$$

Diffusion coefficient For the simplest case of a diagonal diffusion matrix $\mathbf{D}=D \mathbf{1}_{3 \times 3}$ we immediately obtain the well-known diffusion law

$$
\left\langle\vec{r}^{2}\right\rangle=3 \cdot 2 D t
$$

where the prefactor 3 stems from the dimensionality of the problem. The defining equation for the scalar diffusion coefficient $D$ of any diffusing object hence is

$$
\begin{aligned}
D & :=\lim _{t \rightarrow \infty} \frac{\left\langle\vec{r}^{2}\right\rangle}{6 t} \\
& =\left.\frac{1}{6} \lim _{t \rightarrow \infty} \partial_{t^{\prime}}\left\langle\vec{r}^{2}\right\rangle\right|_{t} .
\end{aligned}
$$


The $t \rightarrow \infty$ limit was included since, in general, the diffusion matrix $\mathbf{D}$ may be time-dependent, and so the system's average behavior is being considered. For a polymer chain, for instance, the short-term behavior is further influenced by fast conformational dynamics. Due to the long-time limit, these intrachain effects are separated from the translational diffusion of the chain as a whole, which the diffusion coefficient aims to quantify. For a constant non-diagonal matrix $\mathbf{D}$, we read off that

$$
D=\frac{1}{3} \operatorname{Tr} \mathbf{D}
$$

As a side note, the trace of a matrix is independent of the chosen coordinate system, and so the diffusion coefficient must also be.

Stokes-Einstein relation With equation 2.15 on page 19 , we found that the dynamics of a sphere with radius $a$ is described by the Stokes law $\gamma \mathrm{d}_{t} \vec{r}=\vec{F}$ where $\gamma=6 \pi \eta a$. In that context, the diffusion matrix for this sphere was defined as $\mathbf{D}=T / \gamma \mathbf{1}_{3 \times 3}$. As we just found in the context of stochastic dynamics, the diagonal entries of this diagonal diffusion matrix are in fact equal to the scalar diffusion coefficient $D$. This leads to the famous Stokes-Einstein relation

$$
T=D \gamma=6 D \pi \eta a
$$

The diffusion coefficient $D$ does not only play the role of an inverse friction coefficient for deterministic dynamics, but also dictates the mean square displacement of purely stochastic dynamics. Thus the Stokes-Einstein relation links the statistical properties of a random walk to deterministic, hydrodynamic properties of the object undergoing the random walk.

Hydrodynamic radius In analogy to the case of a diffusing sphere of radius $a$, which the Stokes-Einstein relation sets equals to $T /(6 D \pi \eta)$, here we define any three dimensional object's hydrodynamic radius $R_{H}$ as

$$
R_{H}:=\frac{T}{6 \pi \eta D}
$$

based on its scalar diffusion coefficient. This radius is best understood as the radius of an imagined sphere which diffuses as fast as the object under consideration. We emphasize that it is not assumed that the object itself is a sphere - its hydrodynamic radius is simply a convenient and intuitive measure for its size. For intuition, a sketch of a number of polymer chains together with their hydrodynamic radii is displayed in figure 4.3 on page 97 , where we also demonstrate a numerical method to calculate a polymer chain's $3 \times 3$ diffusion matrix. 


\subsection{Differential geometry}

This section treats the mathematical description of one-dimensional curves living in threedimensional space. These curves may be either continuous or discrete, and they may be framed. A framed curve is a curve which consists not only of its centerline's Cartesian coordinates, but also of information about how it twists around its centerline at each point. Throughout this thesis, we assume curves to be inextensible, meaning that they cannot be stretched or compressed locally. The ultimate purpose of this section is to find analytical expressions for the elastic force $-\vec{\nabla} E$ which can be used for the Langevin equation.

\subsubsection{Continuous space curves}

We first present the general formalism of describing continuous framed space curves as introduced by Kirchhoff [75], later reformulated by Bishop [76] for unframed curves and then supplemented by Langer and Singer [77] to treat framed curves in a Lagrangian manner. This allows for the efficient treatment of framed curves in minimal coordinates.

Description of a continuous curve We parametrize a curve $\vec{r}(s)$ by its arc length $s \in[0, L]$, e.g. $\left|\partial_{s} \vec{r}\right|=1$, where $L$ is the curve's contour length. The unit vector $\vec{t}(s)=\partial_{s} \vec{r}$ is called the curve's tangent vector. Its tip describes a trajectory on the surface of a unit sphere, which is called the indicatrix. Given its starting point $\vec{r}(0)$, the curve can be reconstructed from its indicatrix via

$$
\vec{r}(s)=\vec{r}(0)+\int_{0}^{s} \vec{t}\left(s^{\prime}\right) \mathrm{d} s^{\prime} \quad .
$$

Generally speaking, an indicatrix is more easily parametrized than an arbitrary three-dimensional curve because the tangent vector's normalization is easily taken into account by only allowing rotational changes of $\vec{t}$. This rotation can be conveniently described by introducing two additional vectors $\vec{m}_{1}$ and $\vec{m}_{2}$ which, together with $\vec{m}_{3}:=\vec{t}$, form a right-handed, orthonormal triad. The triad's rotation along the curve is threefold: Any two vectors $\vec{m}_{i}$ and $\vec{m}_{j}$ may simultaneously rotate around $\vec{m}_{k}$ with an angular rate $\Omega_{k}$ (which has the physical dimension angle per length), where $k=1,2,3$ and $i \neq j \neq k \neq i$, such that

$$
\partial_{s}\left(\begin{array}{l}
\vec{m}_{1} \\
\vec{m}_{2} \\
\vec{m}_{3}
\end{array}\right)=\left(\begin{array}{ccc}
0 & \Omega_{3} & -\Omega_{2} \\
-\Omega_{3} & 0 & \Omega_{1} \\
\Omega_{2} & -\Omega_{1} & 0
\end{array}\right) \cdot\left(\begin{array}{l}
\vec{m}_{1} \\
\vec{m}_{2} \\
\vec{m}_{3}
\end{array}\right)
$$

This description of a framed continuous curve is visualized in figure 2.7. Note that the matrix above represents the most general case of a skew-symmetric matrix, and that the sign convention used here makes the rotations around $\vec{m}_{k}$ right-handed if $\Omega_{k}>0$. Equivalently, one may write $\partial_{s} \vec{e}_{k}=\vec{\Omega} \times \vec{e}_{k}$ where $\vec{\Omega}:=\left(\Omega_{1}, \Omega_{2}, \Omega_{3}\right)^{T}$ is called the Darboux vector. 


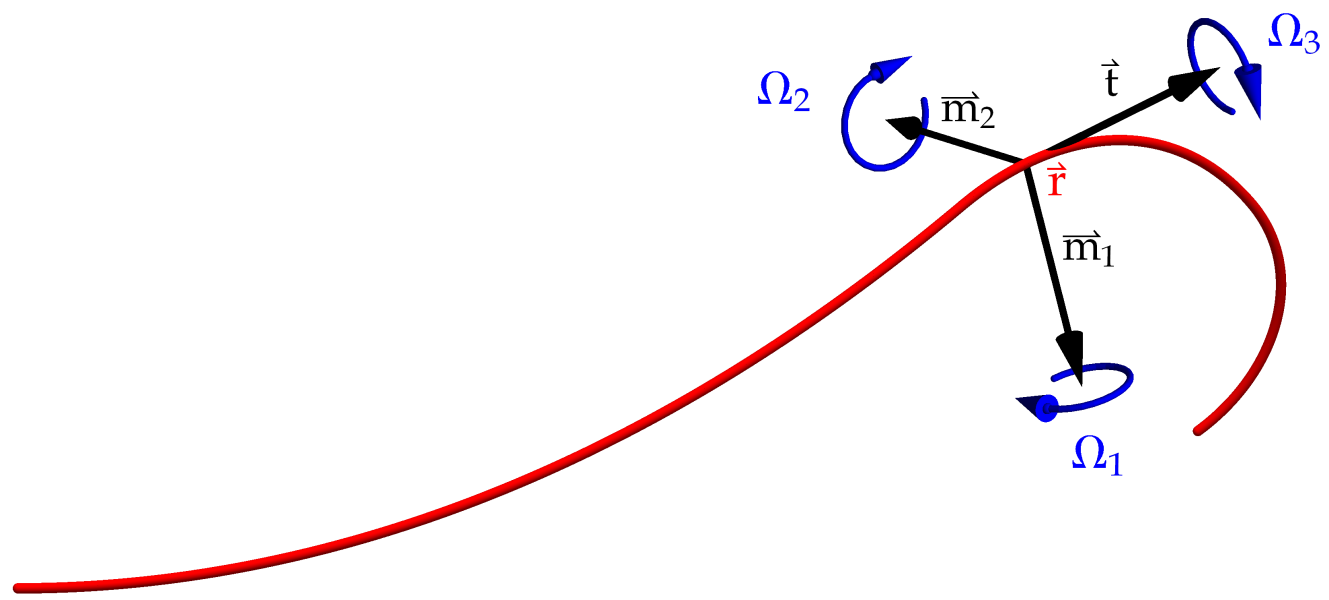

Figure 2.7: A continuous three-dimensional framed curve with its material frame (black triad) shown at one particular point $\vec{r}$ at arc length $s$. The triad rotates around its three vectors with angular rates $\Omega_{k}$, which are shown in blue. Red objects have a physical dimension of length, blue ones have dimension 1 / length, and black ones are dimensionless.

The triad ( $\left.\vec{m}_{1}, \vec{m}_{2}, \vec{m}_{3}\right)$ is also referred to as the material frame. It constitutes the most general description of a framed space curve. In particular, it allows us to encode the twist rate of a physical object via the function $\Omega_{3}$ : the rotation rate around the tangent vector. Imagine for example the track of a roller coaster - its centerline is of interest, and so is its orientation, i.e. the direction the passengers refer to as "up". The rate with which this direction twists around the centerline of the roller coaster track is exactly $\Omega_{3}$. In many applications, however, the twist component of the physical object under consideration is unavailable, or not even of interest. Consider, for instance, noisy microscopy data of thread-like molecules, or the trajectory of a star around the center of the Milky Way. In such cases where only the bare one-dimensional centerline of the curve is given, it is impossible to infer the full material frame simply because of incomplete information.

Bishop frame An alternative frame whose evolution requires only two independent functions can be constructed in the following way. From any material frame one can construct a so-called Bishop [76] (or natural) frame $\left(\vec{b}_{1}, \vec{b}_{2}, \vec{b}_{3}=\vec{m}_{3}=\vec{t}\right)$ which is defined by its lack of a twist rate, i.e. $\vec{b}_{2} \cdot \partial_{s} \vec{b}_{1}=0$. This is easily achieved by introducing the orthonormal vectors $\vec{b}_{1}=\cos \Theta \vec{m}_{1}-\sin \Theta \vec{m}_{2}$ and $\vec{b}_{2}=\sin \Theta \vec{m}_{1}+\cos \Theta \vec{m}_{2}$, where

$$
\Theta(s):=\int_{0}^{s} \Omega_{3}\left(s^{\prime}\right) \mathrm{d} s^{\prime}
$$

is the total twist angle at arc length $s$. 
Thereby, the influence of $\Omega_{3}$ gets undone: the material frame gets "untwisted". It follows that

$$
\vec{b}_{1}+i \vec{b}_{2}=\left(\vec{m}_{1}+i \vec{m}_{2}\right) e^{i \Theta}
$$

such that

$$
\partial_{s}\left(\vec{b}_{1}+i \vec{b}_{2}\right) \stackrel{(2.133)}{=}-\left(\Omega_{2}-i \Omega_{1}\right) e^{i \Theta} \vec{b}_{3}
$$

Conveniently, one also has

$$
\begin{aligned}
\frac{\partial \vec{b}_{3}}{\partial s} & \stackrel{(2.133)}{=} \Omega_{2} \vec{m}_{1}-\Omega_{1} \vec{m}_{2} \\
& =\operatorname{Re}\left(\left(\Omega_{2}-i \Omega_{1}\right) e^{i \Theta} \cdot\left(\vec{m}_{1}-i \vec{m}_{2}\right) e^{-i \Theta}\right)
\end{aligned}
$$

such that we may write

$$
\frac{\partial}{\partial s}\left(\begin{array}{l}
\vec{b}_{1} \\
\vec{b}_{2} \\
\vec{b}_{3}
\end{array}\right)=\left(\begin{array}{ccc}
0 & 0 & -\operatorname{Re}(\Psi) \\
0 & 0 & -\operatorname{Im}(\Psi) \\
\operatorname{Re}(\Psi) & \operatorname{Im}(\Psi) & 0
\end{array}\right) \cdot\left(\begin{array}{l}
\vec{b}_{1} \\
\vec{b}_{2} \\
\vec{b}_{3}
\end{array}\right) .
$$

where we have introduced the complex curvature

$$
\Psi=\left(\Omega_{2}-i \Omega_{1}\right) e^{i \Theta} \quad .
$$

This formula for $\Psi$ allows us to calculate it from a known material frame. However, even without initial knowledge about the material frame, the (unframed) curve $\vec{r}(s)$ can be constructed from any complex function $\Psi$ alone by using equations 2.138 and 2.132. Note also by comparing 2.133 and 2.138 that the Bishop frame is indeed twist-free. Hence, a framed curve can now be efficiently defined in a Lagrangian manner by supplementing a complex function $\Psi$ (which determines the curve's centerline via the Bishop frame) with a scalar function $\Omega_{3}$ (which delivers the angle $\Theta(s)$ between Bishop frame and material frame independently of the centerline). For unframed curves, $\Psi$ suffices.

We make two remarks. Firstly, one may globally (for all $s$ ) rotate $\vec{b}_{1}$ and $\vec{b}_{2}$ around the tangent vector by any globally fixed angle $\phi$, which leads to an identical curve $\vec{r}(s)$ when $\Psi$ is simultaneously multiplied with $e^{i \phi}$. Thus, for a given curve, there are infinitely many twist-free Bishop frames and we have singled out one particular Bishop frame by choosing $\vec{b}_{1}(0)=\vec{m}_{1}(0)$ and $\vec{b}_{2}(0)=\vec{m}_{2}(0)$. Secondly, the modulus of the tangent vector's derivative is called the curvature $\kappa$. Since $\vec{t}=\vec{m}_{3}=\vec{b}_{3}$, it follows that

$$
\kappa=\left|\partial_{s} \vec{t}\right|=\sqrt{\left(\Omega_{1}^{2}+\Omega_{2}^{2}\right)}=|\Psi|=\frac{1}{R}
$$

where $R$ is the radius of the curve's local osculating circle. 
Energy of a continuous rod The bending energy and twisting energy of an elastic rod can be rigorously derived in the framework of elasticity theory. This theory considers macroscopic materials for which the continuum limit applies and bulk properties of the considered material (e.g. the Young's modulus or shear modulus) are meaningful. In this thesis, however, we are not dealing with such materials, but with single molecules whose contour lengths are a few nanometers, and (in a side project) also consider the shapes of flagella whose contour lengths are a few micrometers. Therefore the use of the bending and twisting energy function for macroscopic, elastic rods must clearly be understood as an idealization and we refrain from diving into elasticity theory. The elastic energy of a generic, elastic rod with contour length $L$ reads [111]

$$
E[\vec{\Omega}]=\int_{0}^{L}\left(\frac{\alpha_{1}}{2} \Omega_{1}^{2}+\frac{\alpha_{2}}{2} \Omega_{2}^{2}+\frac{c}{2} \Omega_{3}^{2}\right) \mathrm{d} s
$$

This energy is a functional of the three arbitrary perturbations from a straight, untwisted rod, which is obviously the functional's minimum. It is a local functional which implies that the perturbations at different arc lengths contribute to the total energy independently. Therefore, if required, nonlocal interactions such as steric effects (excluded volume) or electrostatic interactions need to be considered in addition and are not captured by this elastic energy. In general, different elastic constants $\alpha_{1}$ and $\alpha_{2}$ for bending components in the two directions given by the material frame are allowed (imagine a rod with an elliptical cross section for instance). Throughout this thesis, however, the special case $\alpha_{1}=\alpha_{2}=: \alpha$ applies, which allows us to write

$$
E[\vec{\Omega}]=\int_{0}^{L}\left(\frac{\alpha}{2} \kappa^{2}+\frac{c}{2} \Omega_{3}^{2}\right) \mathrm{d} s
$$

The elastic constant $c$ in front of the last term is different from $\alpha$ because it quantifies the response to twisting, not bending.

Heuristically, the functional form of the elastic energy's integrand can easily be justified: Let us interpret it as a Taylor expansion in powers of $\vec{\Omega}$. Constant terms in any physical potential are irrelevant because they do not contribute to that potential's gradient. Furthermore, all appearing terms must not change signs when any $\Omega_{k}$ is replaced by $-\Omega_{k}$. Hence, from this simple argument alone, equation 2.142 is correct up to at least third (inclusive) order.

Dynamics of a continuous curve We do not require any dynamics or variational treatments of continuous rods in this thesis. Suffice to say that the force density acting on the rod equals the functional derivative of the elastic energy with respect to the Cartesian coordinates of the rod's centerline. For a given functional, this functional derivative can be explicitly calculated. For the quadratic functional we are using here, Goldstein et al. [78, 112] derive these terms in explicit form, together with a closed set of partial differential equations describing the spatiotemporal evolution of $\Psi$ and $\Omega_{3}$. We remark that for a quadratic bending energy, boundary conditions apply to $\Psi:\left.\Psi\right|_{0, L}=\left.\partial_{s} \Psi\right|_{0, L}=0$. We elaborate on this in the context of a side project on page 166 . 


\subsubsection{Discrete space curves}

For the discrete analogue of the previously introduced concepts, we present the formalism of Bergou et al [79, 113]. For our purposes, the notation used therein was slightly altered in order to emphasize the physical dimension of the quantities (e.g. a curvature is an angle per length etc.). It should also be noted that we do not aim for the limit of fine discretization, thereby merely approximating a continuous curve. Instead, each vertex of the discrete curve corresponds to a physical monomer (amino acid) whose properties are to be investigated, making the discrete curve an interesting object in its own right rather than a mere approximations to a continuous curve. Furthermore, an inherently discrete framework in minimal coordinates naturally leads us to an efficient numerical treatment.

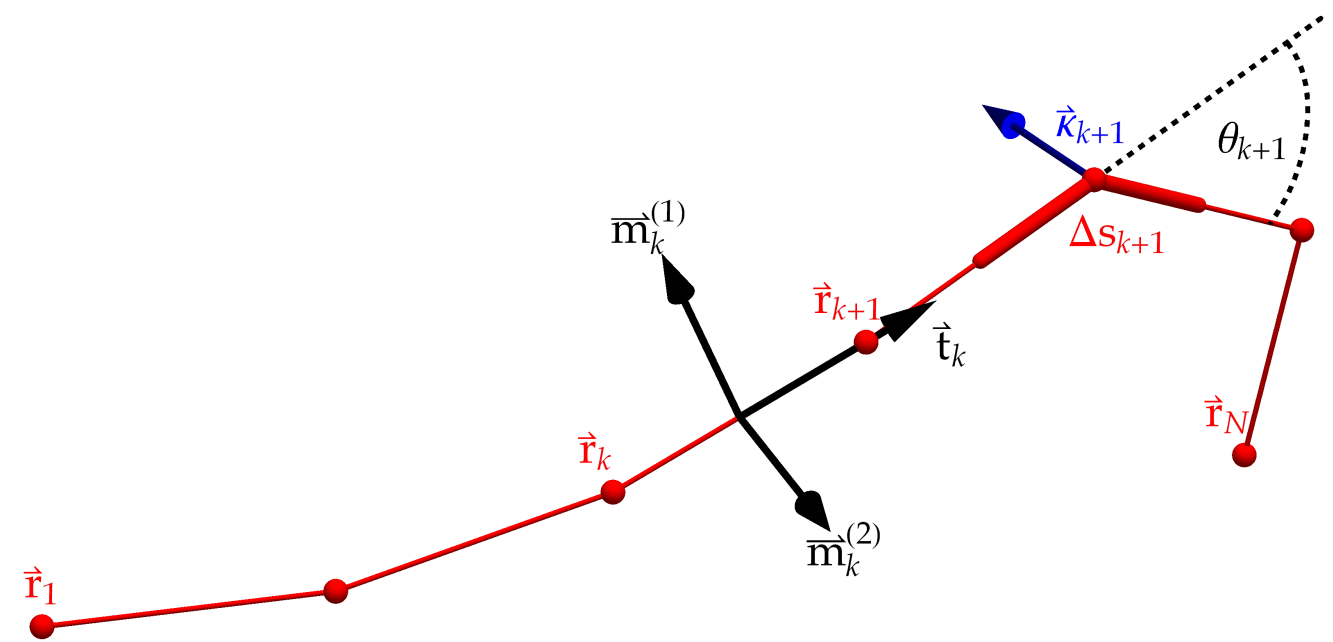

Figure 2.8: A discrete version of the curve in figure 2.7, consisting of $N$ vertices. Hence there are $N-1$ edges and $N-2$ joints (inner vertices). The material frame is naturally associated with an edge, while a bending angle and its curvature (vector) are associated with a joint. The thick red part of the curve around one of its joints shows the arc length $\Delta s$ associated with that joint. Red objects have a physical dimension of length, blue ones have dimension 1 / length, and black ones are dimensionless.

Description of a discrete curve While in principle a continuous chain has an infinite number of degrees of freedom, a discrete chain of finite length does not. It is a polygonal chain given by $N$ Cartesian coordinates

$$
\vec{r}_{i} \quad \text { where } \quad i=1, \ldots, N \quad,
$$

which represent the centerline of the discrete curve. 
A discrete curve (see figure 2.8) consisting of $N$ vertices has $N-1$ edges. These edges are parallel to the normalized tangent vectors

$$
\vec{t}_{i}:=\frac{\vec{r}_{i+1}-\vec{r}_{i}}{\left|\vec{r}_{i+1}-\vec{r}_{i}\right|} \quad \text { where } \quad i=1, \ldots, N-1
$$

Let us further define the arc length associated with an inner vertex as

$$
\Delta s_{i}:=\frac{\left|\vec{r}_{i+2}-\vec{r}_{i+1}\right|+\left|\vec{r}_{i+1}-\vec{r}_{i}\right|}{2} \quad \text { where } \quad i=1, \ldots, N-2 \quad .
$$

Two vectors

$$
\vec{m}_{i}^{(1)} \perp \vec{m}_{i}^{(2)} \perp \vec{t}_{i} \quad \text { where } \quad i=1, \ldots, N-1
$$

are attached to each edge which together with $\vec{m}_{i}^{(3)}=\vec{t}_{i}$ form the material frame at that edge. Analogous to the continuous case, the material frame carries information beyond the mere centerline. Again, we do not explicitly represent it via its vector components, but by signed total twist angles $\Theta_{i}$ (associated with edges) or their discrete derivative, the twist rates $\Omega_{i}^{(3)}$ (associated with inner vertices)

$$
\begin{aligned}
& \Theta_{i}:=\operatorname{sign}\left(\left(\vec{b}_{i}^{(1)} \times \vec{m}_{i}^{(1)}\right) \cdot \vec{t}_{i}\right) \cdot \arccos \left(\vec{b}_{i}^{(1)} \cdot \vec{m}_{i}^{(1)}\right) \quad \text { where } \quad i=1, \ldots, N-1 \\
& \Omega_{i}^{(3)}:=\frac{\Theta_{i+1}-\Theta_{i}}{\Delta s_{i}} \quad \text { where } i=1, \ldots, N-2
\end{aligned}
$$

relative to the Bishop frame, thereby avoiding redundant information and stiff constraints. Instead, the material frame is easily reconstructed from the Bishop frame by rotating it around the respective tangent vectors with angles $\Theta_{i}$.

Parallel transport In order to establish the notion of a Bishop frame attached to a discrete curve, we first introduce the concept of parallel transport. When a tangent vectors rotates from an initial state $\vec{t}^{\text {before }}$ to a new state $\vec{t}^{\text {after }}$, parallel transporting an arbitrary vector $\vec{v}^{\text {before }}$ simply means rotating it along with the tangent vector; it gets rotated around the axis $\vec{a}$ by an angle $\phi$ given by

$$
\begin{aligned}
\vec{a} & =\frac{\vec{t}^{\text {before }} \times \vec{t}^{\text {after }}}{\left|\vec{t}^{\text {before }} \times \vec{t}^{\text {after }}\right|} \\
\text { and } \quad \phi & =\arccos \left(\vec{t}^{\text {before }} \cdot \vec{t}^{\text {after }}\right) \quad .
\end{aligned}
$$

Let us denote parallel transport via a multiplication with a rotation matrix $P$ as follows:

$$
P\left(\vec{t}^{\text {before }}, \vec{t}^{\text {after }}\right) \cdot \vec{v}^{\text {before }}=\vec{v}^{\text {after }}
$$


Bishop frame The axis $\vec{a}$ is perpendicular to both $\vec{t}^{\text {before }}$ and $\vec{t}^{\text {after }}$. Thus, when we parallel transport a vector along the curve such that $\vec{t}^{\text {before }}=\vec{t}_{i}$ and $\vec{t}^{\text {after }}=\vec{t}_{i+1}$, the applied rotation does not correspond to a twisting rate $\Omega_{3}$ (which rotates around the tangential direction, see equation 2.133). Therefore, the (twist-free) Bishop frame of a given curve is easily constructed via repeated parallel transport:

$$
\vec{b}_{i}^{(1)}=\left(\prod_{k=1}^{i-1} P\left(\vec{t}_{k}, \vec{t}_{k+1}\right)\right) \cdot \vec{b}_{1}^{(1)} \quad \text { where } \quad i=2, \ldots, N-2 \quad .
$$

This equation is, of course, best solved in an iterative manner after an initial triad $\left(\vec{b}_{1}^{(1)}, \vec{b}_{1}^{(2)}, \vec{t}_{1}\right)$ is chosen. The third vector of each triad immediately follows from its orthonormality. Like in the continuous setting, we choose the initial Bishop frame to coincide with the material frame,

$$
\vec{b}_{1}^{(j)}=\vec{m}_{1}^{(j)} \quad \text { where } \quad j=1,2 \quad,
$$

but in principle there are infinitely many Bishop frames which can be transformed into each other by globally rotating around the tangent direction.

Discrete curvature Next we define the curvature $\kappa_{i}$ associated with an inner vertex. The discrete curvature must converge to the continuous curvature $\kappa(s)=\left|\partial_{s} \vec{t}\right|$ in the limit $N \rightarrow \infty$ at fixed contour length. It must hence have the physical dimension of angle per length. Since in said limit, the bending angles

$$
\theta_{i}:=\arccos \left(\vec{t}_{i} \cdot \vec{t}_{i+1}\right) \quad \text { where } \quad i=1, \ldots, N-2
$$

become infinitesimal, it is sufficient that $\Delta s \kappa_{i} \propto \theta_{i}+\mathscr{O}\left(\theta_{i}^{2}\right)$ for proper convergence to the continuous limit alone. For a continuous curve which is everywhere slightly displaced by an offset $\epsilon$ into its normal direction, Steiner's theorem relates its curvature to its change in contour length via $\Delta L=\epsilon \int \kappa \mathrm{d} s$. Motivated by this simple theorem, different definitions of discrete curvature are used in literature [114]: $\kappa^{(1)}=\theta / \Delta s, \kappa^{(2)}=2 \sin (\theta / 2) / \Delta s$ and $\kappa^{(3)}=2 \tan (\theta / 2) / \Delta s$. All these definitions correctly converge towards the continuous curve while reproducing some of its properties, but unfortunately there is no single discrete curvature which preserves all properties of the continuous curve simultaneously. For instance, the continuous curvature of a closed, planar curve always adds up to a multiple of $2 \pi$ when integrated over the curve, and only $\kappa^{(1)}$ has this property in the discrete setting. Conservation of the curve's center of mass under so-called curve-shortening flows is only given when $\kappa^{(2)}$ is chosen as the discrete curvature. The bottom line is that a choice must be made depending on which properties of the discrete curve are desired for the application at hand. For our purposes, we choose the third definition

$$
\kappa_{i}:=\frac{2 \tan \left(\theta_{i} / 2\right)}{\Delta s_{i}} \quad \text { where } \quad i=1, \ldots, N-2
$$


for two reasons. Firstly, using this curvature definition, the probability distribution of a discrete chain's end-to-end distance (in a heat bath) converges faster to the continuous worm-like chain limit than for the other two definitions. We demonstrate this numerically in appendix A, and the worm-like chain is introduced on page 66 . Secondly, using finite discretization, the tangent-based curvature has the property that it diverges as $\theta \rightarrow \pm \pi$, which - due to the chain's elastic response to deformations - ensures that the bending angles cannot exceed $\pi$ and the bond vector cannot make "a full turn", which would be unphysical.

Let us now introduce the curvature binormal vector $\vec{\kappa}_{i}$ as

$$
\vec{\kappa}_{i}=\frac{1}{\Delta s_{i}} \cdot \frac{2 \vec{t}_{i} \times \vec{t}_{i+1}}{1+\vec{t}_{i} \cdot \vec{t}_{i+1}} \quad \text { where } \quad i=1, \ldots, N-2
$$

which indeed satisfies $\left|\vec{\kappa}_{i}\right|=\kappa_{i}$ and is by construction parallel to the axis around which the tangent vector is rotated. Therefore $\vec{\kappa}_{i}$ may be utilized to find the bending rates into the two direction given by the material frame vectors. This is analogous to disentangling $\partial_{s} \vec{t}$ into $\Omega_{1}$ and $\Omega_{2}$ in the continuous setting, see equation 2.133. Note that while the material and Bishop frame are associated with edges, bending angles and thus curvature values are associated with inner vertices. Therefore, the angular bending rates

$$
\Omega_{i}^{(j)}=(-1)^{j+1} \vec{\kappa}_{i} \cdot \frac{\vec{m}_{i}^{(j)}+\vec{m}_{i+1}^{(j)}}{2} \quad \text { where } \quad i=1, \ldots, N-2 \quad \text { and } \quad j=1,2
$$

are defined by projecting the curvature binormal vector onto the average directions given by the two adjacent material frames. The minus sign is introduced to maintain the convention that the $\Omega_{i}^{(j)}$ describe right-handed rotations around $\vec{m}_{i}^{(j)}$.

Energy of a discrete rod The elastic energy of a discrete, elastic rod reads

$$
E=\sum_{i=1}^{N-2}\left(\frac{\alpha}{2}\left(\Omega_{i}^{(1)}-\bar{\Omega}_{i}^{(1)}\right)^{2}+\frac{\alpha}{2}\left(\Omega_{i}^{(2)}-\bar{\Omega}_{i}^{(2)}\right)^{2}+\frac{c}{2}\left(\Omega_{i}^{(3)}\right)^{2}\right) \Delta s_{i}
$$

and can be interpreted as a discretized version of the previously discussed elastic energy of a continuous rod. Here, we have already assumed that the bending response is isotropic $\left(\alpha_{1}=\right.$ $\alpha_{2}=\alpha$ ), which corresponds to a circular cross section of the elastic rod. Furthermore, we have introduced the reference curvatures $\bar{\Omega}_{i}^{(j)}$ which are not variables describing the curve, but fixed material properties. They allow us to model elastic rods whose energetic minimum (equilibrium conformation) is not necessarily a straight rod. The arbitrary energetic minimum is still assumed to be untwisted, i.e. $\bar{\Omega}_{i}^{(3)}=0$. For example, constant reference curvatures refer to a circular arc as the rod's energetic minimum. It must be noted that the reference curvatures are not only associated with a centerline conformation, but also with a reference material frame, which in our untwisted reference state is a Bishop frame. 
Elastic forces The elastic forces acting on vertex $i$ are given by the negative gradient of the elastic energy $E$ with respect to $\vec{r}_{i}$. Defining $\vec{e}_{i}:=\vec{r}_{i+1}-\vec{r}_{i}, \chi:=1+\vec{t}_{i} \cdot \vec{t}_{i+1}, \tilde{\vec{t}}_{i}:=\left(\vec{t}_{i}+\vec{t}_{i+1}\right) / \chi$ and $\tilde{\vec{m}}_{i}^{(j)}:=\left(\vec{m}_{i}^{(j)}+\vec{m}_{i+1}^{(j)}\right) / \chi$, the only nontrivial gradients required to calculate these forces are

$$
\begin{aligned}
\frac{\partial \Omega_{i}^{(1)}}{\partial \vec{e}_{i}} & =\frac{-\Omega_{i}^{(1)} \tilde{\vec{t}}_{i}+\vec{t}_{i+1} \times \tilde{\vec{m}}_{i}^{(2)}}{\left|\vec{e}_{i}\right|}, \\
\frac{\partial \Omega_{i}^{(1)}}{\partial \vec{e}_{i+1}} & =\frac{-\Omega_{i}^{(1)} \tilde{\vec{t}}_{i}-\vec{t}_{i} \times \tilde{\vec{m}}_{i}^{(2)}}{\left|\vec{e}_{i+1}\right|}, \\
\frac{\partial \Omega_{i}^{(2)}}{\partial \vec{e}_{i}} & =\frac{-\Omega_{i}^{(2)}}{\tilde{\vec{t}}_{i}-\vec{t}_{i+1} \times \tilde{\vec{m}}_{i}^{(1)}} \\
\frac{\partial \Omega_{i}^{(2)}}{\partial \vec{e}_{i+1} \mid} & =\frac{-\Omega_{i}^{(2)} \tilde{\vec{t}}_{i}+\vec{t}_{i} \times \tilde{\vec{m}}_{i}^{(1)}}{\left|\vec{e}_{i+1}\right|}, \\
\frac{\partial \Omega_{i}^{(3)}}{\partial \vec{e}_{i}} & =\frac{\vec{\kappa}_{i}}{2\left|\vec{e}_{i}\right|} \\
\text { and } \quad \frac{\partial \Omega_{i}^{(3)}}{\partial \vec{e}_{i+1}} & =\frac{\vec{\kappa}_{i}}{2\left|\vec{e}_{i+1}\right|}
\end{aligned}
$$

Throughout this thesis, we are assuming inextensible curves, e.g. $\Delta s_{i}=$ const..

One important special case is that of a naturally straight curve without twisting elasticity., i.e. $\bar{\Omega}_{i}^{(1)}=\bar{\Omega}_{i}^{(2)}=c=0$. In this case, one has $\left(\Omega_{i}^{(1)}\right)^{2}+\left(\Omega_{i}^{(2)}\right)^{2}=\left(\vec{\kappa}_{i}\right)^{2}=\kappa_{i}^{2}$ such that the elastic energy $E$ reduces to the bending energy

$$
E_{b}=\frac{\alpha}{2} \sum_{i=1}^{N-2} \vec{\kappa}_{i}^{2} \Delta s_{i}
$$

The only nontrivial terms required to calculate its gradient are

$$
\begin{aligned}
\vec{\nabla}_{i} \cdot \vec{\kappa}_{i}^{T} & =\frac{2 \mathbf{E}_{i+1}+\vec{\kappa}_{i} \cdot \vec{e}_{i+1}^{T}}{\left|\vec{e}_{i}\right| \cdot\left|\vec{e}_{i+1}\right|+\vec{e}_{i} \cdot \vec{e}_{i+1}} \\
\vec{\nabla}_{i+1} \cdot \vec{\kappa}_{i}^{T} & =-\left(\vec{\nabla}_{i}+\vec{\nabla}_{i+2}\right) \cdot \kappa_{i} \\
\text { and } \quad \vec{\nabla}_{i+2} \cdot \vec{\kappa}_{i}^{T} & =\frac{2 \mathbf{E}_{i}-\vec{\kappa}_{i} \cdot \vec{e}_{i}^{T}}{\left|\vec{e}_{i}\right| \cdot\left|\vec{e}_{i+1}\right|+\vec{e}_{i} \cdot \vec{e}_{i+1}}
\end{aligned}
$$

where $\mathbf{E}_{j} \cdot \vec{x}=\vec{e}_{j} \times \vec{x}$. Note that these expressions are matrices and do not require knowledge of the material or Bishop frame. Therefore, in applications where this special case applies, one does not need to keep track of any adapted frames at all, but can describe the chain's dynamics in terms of the Cartesian coordinates of its vertices alone. 
Dynamics of a discrete rod So far, we have dealt with the description of an elastic rod at a fixed point in time. Later on, the dynamics of discrete chains will be simulated and thus we need to study how the introduced quantities change when a chain is moved. Let us consider an initial discrete rod at time $t_{1}$, for which all geometric quantities have already been calculated, being transformed into a new conformation given at time $t_{2}>t_{1}$. This transformation is described by some physical equation of motion such as the Langevin equation to which the elastic forces contribute. Calculating the new geometric quantities from the new coordinates $\vec{r}_{i}\left(t_{2}\right)$ is straightforward in all cases except for one: the material frame. Physically speaking, the material frame at edge $i$ is fixed to that edge, which is being rotated from $\vec{r}_{i+1}\left(t_{1}\right)-\vec{r}_{i}\left(t_{1}\right)$ to $\vec{r}_{i+1}\left(t_{2}\right)-\vec{r}_{i}\left(t_{2}\right)$. The material frames along the curve are thus undergoing the same rotations as their respective tangent vectors which are parallel to that edge. Conveniently, this transformation is described via parallel transport as

$$
\vec{m}_{i}^{(j)}\left(t_{2}\right)=P\left(\vec{t}_{i}\left(t_{1}\right), \vec{t}_{i}\left(t_{2}\right)\right) \cdot \vec{m}_{i}^{(j)}\left(t_{1}\right)
$$

Therefore, material frames at a fixed edge $i$ can be found iteratively via parallel transport in time. On the other hand, Bishop frames at a fixed time $t$ can be found iteratively via parallel transport in space. Generally speaking, even if material frame and Bishop frame coincide at an initial time $t_{0}$, any transformation $\mathrm{d}_{t} \vec{r}$ which is not perpendicular to the twist gradient $\vec{\nabla} \Omega_{3}$ drives them apart. In other words: out-of-plane bending induces twist.

We reiterate that in the mentioned special case $\bar{\Omega}_{i}^{(1)}=\bar{\Omega}_{i}^{(2)}=c=0$, keeping track of the material frame during a simulation is not required. 


\subsection{Polymer theory}

A polymer is a linear chain of many (poly) units (mer) which may be identical (homopolymer) or differ from each other (heteropolymer).

Different polymer models can all be understood within the framework of statistical mechanics $[115,116]$. One specifies variables by which the polymer's state is to be represented and then assumes a probability distribution for these variables. This is equivalent to assuming an energy function because of the simplicity of the Boltzmann distribution. From the probability distribution, one then calculates statistical properties of the polymer which are of interest, e.g. its mean squared end-to-end distance $\left\langle R^{2}\right\rangle$ or persistence length $l_{P}$. For later reference in this thesis, a number of mathematical models of individual polymer molecules will now be introduced.

Freely jointed chain A freely jointed chain (FJC) describes a random walk in three-dimensional space. This random walk consists of $N$ steps which are statistically independent of each other. Each step $\Delta \vec{r}_{i}$ has a fixed step length $d$ and points into a random direction, each of which are equally likely such that

$$
\begin{aligned}
p\left(\Delta \vec{r}_{i}\right) & =\frac{1}{4 \pi d^{2}} \delta\left(\left|\Delta \vec{r}_{i}\right|-d\right) \\
\left\langle\Delta \vec{r}_{i}\right\rangle & =0 \\
\text { and } \quad\left\langle\Delta \vec{r}_{i} \cdot \Delta \vec{r}_{j}\right\rangle & =d^{2} \delta_{i j} .
\end{aligned}
$$

The contour of the polymer is the trajectory described by the random walk. It starts in the origin and ends at the point $\vec{R}$ which is

$$
\vec{R}=\sum_{i=1}^{N} \Delta \vec{r}_{i}
$$

One trivially has $\langle\vec{R}\rangle=0$, but the polymer still has finite size which is commonly quantified by $\sqrt{\left\langle\vec{R}^{2}\right\rangle}$ :

$$
\begin{aligned}
\left\langle\vec{R}^{2}\right\rangle & =\sum_{i j}^{N}\left\langle\Delta \vec{r}_{i} \cdot \Delta \vec{r}_{j}\right\rangle=N d^{2} \\
\text { and } \quad \sqrt{\left\langle\vec{R}^{2}\right\rangle} & =\sqrt{N} d .
\end{aligned}
$$

This square-root behavior with the number of steps is the hallmark of diffusion - we have already seen it in the diffusion equation (the variance obeyed $\sigma^{2}=t$ ) and in the Wiener process $\left(\left\langle\Delta W^{2}\right\rangle=\Delta t\right)$. The analogy is clear: Before, we considered a random walk in time and here we consider a random walk in space, but the underlying concepts are identical.

The FJC is clearly an oversimplified polymer model, but the simple scaling law $\left(R \propto N^{0.5}\right)$ which it predicts is surprisingly accurate and universal as long as the considered polymer is long enough for the spatial orientation of its sub-units to indeed be random and uncorrelated. In literature, $d$ is also known as the Kuhn length, and the FJC as the Kuhn chain. 
Gaussian chain Due to the central limit theorem, the probability distribution of the end-toend vector $\vec{R}$ is always Gaussian for large values of $N$. The Gaussian chain is thus the limiting case of any polymer model, and in particular of the FJC. The central limit theorem states that variance and mean of the individual $\Delta \vec{r}_{i}$ enter the Gaussian end-to-end distribution in an additive way. We need to be careful, however, since we are dealing with vector quantities. Let us thus consider each component individually. For a single component $\Delta r_{i}^{(k)}$ of $\Delta \vec{r}_{i}$ one has (from equation 2.161) $\left\langle\Delta r_{i}^{(k)}\right\rangle=0$ and $\left\langle\left(\Delta r_{i}^{(k)}\right)^{2}\right\rangle=d^{2} / 3$. The components of the end-to-end vector therefore satisfy $\left\langle R^{(k)}\right\rangle=0$ and $\left\langle\left(R^{(k)}\right)^{2}\right\rangle=N d^{2} / 3=\left\langle\vec{R}^{2}\right\rangle / 3$. Multiplying all three components together yields the Gaussian distribution for the end-to-end vector:

$$
\lim _{N \rightarrow \infty} p(\vec{R})=\frac{1}{\left(2 \pi\left\langle\vec{R}^{2}\right\rangle / 3\right)^{3 / 2}} \exp \left(-\frac{3}{2} \frac{\vec{R}^{2}}{\left\langle\vec{R}^{2}\right\rangle}\right) \quad .
$$

Comparing this with the Boltzmann distribution, it is easy to read off that the free energy associated with a particular value of $\vec{R}$ is

$$
F=T \cdot \frac{3}{2} \frac{\vec{R}^{2}}{\left\langle\vec{R}^{2}\right\rangle}
$$

A quadratic energy results in a linear force and since linear equations are simple, Gaussian chain models enjoy great popularity in polymer dynamics. They are valid for sufficiently long polymers: When each of their monomers $\vec{r}_{i}$ represents enough physical monomers for the central limit theorem to apply, then they experience two forces proportional to $\Delta \vec{r}_{i-1}$ and $\Delta \vec{r}_{i}$ respectively. The simplest Gaussian chain is that which neglects hydrodynamic interactions, and is called the Rouse model [15].

Flory chain A Flory chain is a polymer model which takes into account excluded volume effects. Excluded volume effects state that non-neighboring units cannot overlap sterically. In order to study the scaling behavior

$$
\left\langle\vec{R}^{2}\right\rangle \propto N^{v}
$$

Flory [69, 115, 116] made a simple scaling argument which we repeat here. We consider a sufficiently long FJC (consisting of $N$ bonds) whose end-to-end distance is fixed to $|\vec{R}|=R$. In equation 2.165 , we have already found the free energy associated with the mesostate defined by the fixed value of $R=|\vec{R}|$ :

$$
F_{1}(R)=T \cdot \frac{3}{2} \frac{R^{2}}{N d^{2}}
$$

This free energy is of a purely entropic nature since there is no energy term present in the FJC. 
Now we add a second free energy contribution $F_{2}$ which is of a purely energetic nature and takes into account excluded volume effects. The excluded volume effects are of a repulsive nature and are assumed to be pairwise interactions between monomers such that

$$
F_{2}(R) \propto V \cdot \rho^{2} \propto \frac{N^{2}}{R^{3}}
$$

where $V \propto R^{3}$ is the volume occupied by the chain and $\rho \propto N / V$ is the monomer density within $V$. Flory's idea was to change the perspective and treat this FJC with end-to-end distance $R$ as a sphere of radius $R$ whose energy is given by

$$
E(R)=F_{1}(R)+F_{2}(R) \quad .
$$

A mechanical force balance $\partial_{R} E(R) \stackrel{!}{=} 0$ then delivers the simple scaling law

$$
R \propto N^{0.6} \quad
$$

Although Flory's argument is somewhat hand-waving as it disregards the inherently stochastic nature of the system, the scaling law he acquired is surprisingly accurate - more sophisticated considerations (see pages 27-29 in [115] and references given therein) deliver the value $v=0.588$. As a side note, Flory's result is exact in one, two and 4 dimensions. In any case, $0.5<0.6<1$ and hence excluded volume effects make the chain swell compared to a purely random walk $\left(R \propto N^{0.5}\right)$, but we are still well below the scaling behavior of a straight line $(R \propto N)$.

Worm-like chain The worm-like chain [17] (WLC) is a famous polymer model which takes into account bending stiffness and is thus a semiflexible chain. In contrast to the polymer models we treated before, the WLC is a continuous curve. It is an elastic rod embedded in a heat bath. Its geometry and elastic properties can thus be described as before (see section 2.3.1) and its bending energy reads

$$
E_{\mathrm{WLC}}=\frac{\alpha}{2} \int_{0}^{L}\left|\partial_{s} \vec{t}\left(s^{\prime}\right)\right|^{2} \mathrm{~d} s^{\prime} \quad .
$$

The difference to a classical elastic rod is that the heat bath surrounding it leads to thermal activation of the WLC such that the probability density value for a particular conformation represented by the function $\vec{t}(s)$ is given by the Boltzmann distribution

$$
p[\vec{t}(s)] \propto \exp \left(-\frac{E_{\mathrm{WLC}}}{T}\right)=\exp \left(-\frac{\alpha}{2 T} \int_{0}^{L}\left|\partial_{s} \vec{t}\left(s^{\prime}\right)\right|^{2} \mathrm{~d} s^{\prime}\right) \quad .
$$

In other words, in contrast to a macroscopic elastic rod, it minimizes its free energy, not its energy. In appendix $\mathrm{D}$ we show that in thermal equilibrium, the autocorrelation of the tangent direction of the WLC decays exponentially:

$$
\left\langle\vec{t}\left(s_{1}\right) \cdot \vec{t}\left(s_{2}\right)\right\rangle=\exp \left(-\frac{\left|s_{1}-s_{2}\right|}{l_{P}}\right) \quad .
$$


Here, the persistence length

$$
l_{P}=\frac{\alpha}{T}
$$

was defined. It dictates the length scale on which the tangential direction becomes uncorrelated along the curve and is thus an important quantity which quantifies how quickly a polymer bends. While a rope thrown on the ground may have a persistence length of one meter, a protein's persistence length is on the order of one nanometer.

The WLC's end-to-end distance

$$
\vec{R}=\int_{0}^{L} \vec{t}(s) \mathrm{d} s
$$

obeys

$$
\begin{aligned}
\left\langle\vec{R}^{2}\right\rangle & =\int_{0}^{L} \int_{0}^{L}\left\langle\vec{t}\left(s_{1}\right) \cdot \vec{t}\left(s_{2}\right)\right\rangle \mathrm{d} s_{1} \mathrm{~d} s_{2} \\
& =\int_{0}^{L} \int_{0}^{L} \exp \left(-\frac{\left|s_{1}-s_{2}\right|}{l_{P}}\right) \mathrm{d} s_{1} \mathrm{~d} s_{2} \\
& =2 N_{P} l_{P}^{2}-2 l_{P}^{2}\left(1-e^{-N_{P}}\right)
\end{aligned}
$$

where $N_{P}:=L / l_{P}$ is the number of persistence lengths fitting into the chain's contour length. This expression consists of two terms. For $N_{P} \ll 1$ we can Taylor expand the exponential function in the second term. The result is that $\left\langle\vec{R}^{2}\right\rangle \stackrel{N_{P} \ll 1}{\longrightarrow} L^{2}$, which is the limit of a straight rod. This makes sense because a WLC which is much shorter than its persistence length has a constant tangent direction according to equation 2.173. The first term dominates when $N_{P} \gg 1$, i.e. when the WLC is much longer than its persistence length, which is the diffusive limit of a FJC. Comparison with the FJC, together with $L=N d=N_{P} l_{P}$, yields the translation rule

$$
d=2 l_{P} \quad .
$$

In other words: a WLC with length $L$ and persistence length $l_{P}$ has the same contour length and mean-squared end-to-end distance as a FJC with $L /\left(2 l_{P}\right)$ bonds of length $2 l_{P}$. The number $L /\left(2 l_{P}\right)$ must therefore be large for the central limit theorem to make the WLC's end-to-end vector be Gaussian. When excluded volume effects are included in a WLC, a non-local energy term is added to the bending energy. This term depends on all tangential vectors simultaneously in a non-trivial manner. The simple monoexponential form of $\left\langle\vec{t}\left(s_{1}\right) \cdot \vec{t}\left(s_{2}\right)\right\rangle$ can thus no longer be expected to hold true. In this case, we redefine the persistence length as

$$
l_{P}=\frac{1}{1-\langle\vec{t}(0) \cdot \vec{t}(L)\rangle} \int_{0}^{L}\left\langle\vec{t}(0) \cdot \vec{t}\left(s^{\prime}\right)\right\rangle \mathrm{d} s^{\prime} \quad,
$$

which coincides with the first definition in the absence of excluded volume effects. The denominator's purpose is to compensate the fact that $L$ may be finite, in which case the integral is too small because it has not summed up all the area below the exponential function. 
Discrete worm-like chain A discrete WLC is also called a Kratky-Porod chain [68]. It can alternatively be understood as either a FJC which is supplemented with a bending energy, or as a discrete elastic rod in a heat bath. Due to the additive nature of the discrete bending energy $\mathrm{E}$, the Boltzmann distribution factorizes and, remembering that $\kappa_{i}=2 \tan \left(\theta_{i} / 2\right) / \Delta s_{i}$, we may consider each bending angle $\theta_{i}$ individually:

$$
\begin{aligned}
& p(\vec{\theta}) \propto \exp \left(-\frac{E}{T}\right)=\exp \left(-\frac{\alpha}{2 T} \sum_{i=1}^{N-2} \kappa_{i}^{2} \Delta s_{i}\right)=\prod_{i=1}^{N-2} \exp \left(-\frac{\alpha}{2 T} \kappa_{i}^{2} \Delta s_{i}\right) \\
& p\left(\theta_{i}\right) \propto \exp \left(-\frac{\alpha}{2 T} \kappa_{i}^{2} \Delta s_{i}\right) .
\end{aligned}
$$

Note that no energy is associated with bond rotations of a tangent vector around the previous tangent vector (azimuthal or torsion angle). As a result, analogous to the continuous WLC, we have (see appendix D)

$$
\left\langle\vec{t}_{1} \cdot \vec{t}_{k+1}\right\rangle=\exp \left(-\frac{k d}{l_{P}}\right)
$$

where we set all $\Delta s_{i}=d$. In the discrete case, the simple relation $l_{P}=\frac{\alpha}{T}$ does not hold true, however. Instead, setting $k=1$ in the equation above yields

$$
\begin{aligned}
\langle\cos \theta\rangle & =\exp \left(-\frac{d}{l_{P}}\right) \\
\Leftrightarrow l_{P} & =-\frac{d}{\ln \langle\cos \theta\rangle}
\end{aligned}
$$

where $(y:=\cos \theta)$

$$
\langle\cos \theta\rangle=\frac{\int_{-1}^{1} y \cdot \exp \left(-\frac{\sigma}{2 d}\left(2 \tan \left(\frac{\arccos y}{2}\right)\right)^{2}\right) \mathrm{d} y}{\int_{-1}^{1} \exp \left(-\frac{\sigma}{2 d}\left(2 \tan \left(\frac{\arccos y}{2}\right)\right)^{2}\right) \mathrm{d} y} .
$$

Here, we defined $\sigma$ as

$$
\alpha=T \sigma \quad,
$$

and refer to $\sigma$ instead of $\alpha$ as the bending stiffness even though it is a length. Solving these integrals analytically is hopeless, but one can easily calculate the function $l_{P}(\sigma / d)$ numerically, which we have done in figure 2.9. As expected, it turns out that $l_{P} / \sigma \rightarrow 1$ as $\sigma / d \rightarrow \infty$, which is the limit towards the continuous WLC.

In the case of a discrete chain with excluded volume effects, we define the persistence length analogously to our definition in the continuous setting: as the integral over $\left\langle\vec{t}_{1} \cdot \vec{t}_{k+1}\right\rangle$. The integral of this discrete function is evaluated using the Simpson method for discrete integration. 


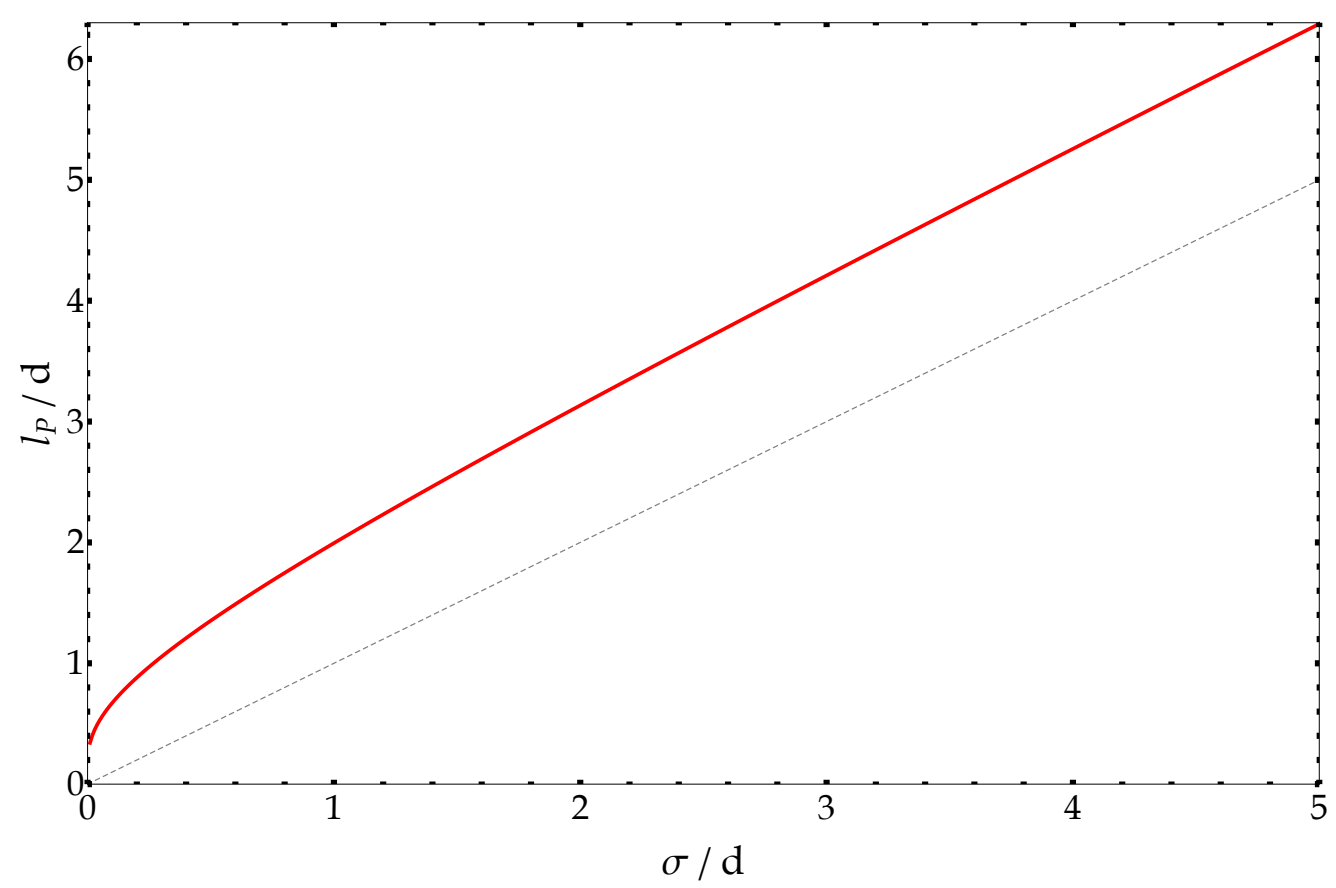

Figure 2.9: The relationship between the bending stiffness $\sigma$ and persistence length $l_{P}$ (both in units of the bond length $d$ ) in a discrete WLC without excluded volume interactions (shown in red). The dashed diagonal line has slope one and is only shown for visual purposes. In the limit of a very stiff chain, one has many chain vertices per persistence length of the chain, thus arriving at the limit of a continuous WLC in which case $l_{P}=\sigma$. 


\subsection{Experimental methods}

In this section we introduce the experimental methods whose results we are modeling in this thesis. It is not meant as a detailed description, but is solely focused on the concepts, as I have not performed any experiments myself.

\subsubsection{Single-molecule spectroscopy (SMS)}

Fluorescence is the basic photo-physical mechanism which all experimental methods below are based on. It is described in the Jablonski diagram (see figure 2.10) and made use of by chemically labeling single molecules (e.g. proteins) with a so-called fluorophore (also called a dye) at their chain end. The fluorophore is typically much smaller than the molecule itself but larger than an amino acid, and unlike the molecule itself it is fluorescent, i.e. it emits light. Having the fluorophores as optically visible reporters attached to the otherwise invisible molecules of interest allows the investigation of the molecules via single-molecule spectroscopy (SMS). A typical experimental setup for SMS is shown in figure 2.11. Possible samples are those described below (PET and dynaMIET), for both of which the experimental setup does not need to be altered. The data collected by the detector(s) is analyzed using fluorescence correlation spectroscopy.

\subsubsection{Fluorescence correlation spectroscopy (FCS)}

Fluorescence correlation spectroscopy (FCS) is not an experimental setup or a physical effect, but a data-analysis technique [84-86]. Suppose that we have at hand experimental data recorded by the detectors in figure 2.11 - they have detected a large number of individual photons $p=1, \ldots, P$ arriving at times $t_{1}, \ldots, t_{P}$. The basic idea of FCS is to histogram the arrival time differences of all photon pairs. The resulting histogram (a function of the time difference) yields information about the temporal dynamics of individual photon sources (molecules), and now we demonstrate why this is the case.

Suppose that, for whatever reason, the detected intensity (rate of photon emission) of a single molecule is time-dependent. Such reasons could be that the molecule diffuses out of the excitation focus, or that the PET or MIET effect, which will be discussed in the following subsections, takes place. Let us thus denote the intensity of molecule number $m$ as $I_{m}(t)$ where $m=1, \ldots, M$. Due to thermal fluctuations, this function will be the realization of a stochastic process and therefore it only makes sense to study statistical properties of $I_{m}(t)$ such as its autocorrelation function

$$
C_{m}(\tau)=\frac{\left\langle\Delta I_{m}(t) \cdot \Delta I_{m}(t+\tau)\right\rangle_{t}}{\sigma_{m}^{2}}
$$

where $\Delta I_{m}(t)=I_{m}(t)-\langle I\rangle$ and $\sigma_{m}^{2}=\left\langle\left(\Delta I_{m}\right)^{2}\right\rangle$. The functional form of $C_{m}(\tau)$ contains information about the molecule's dynamics and can be compared with simulation results. It is thus the function that we are interested in. 
Experimentally, however, one does not only detect photons from molecule $m$ but from a large number $M$ (the average number of molecules in the excitation volume) of molecules simultaneously. The measured intensity hence is

$$
I(t)=\sum_{m=1}^{M} I_{m}(t) \propto \sum_{p=1}^{P} \delta\left(t-t_{p}\right)
$$

such that when a photon is detected, it is unclear which molecule it was emitted by. Let us thus define $C(\tau), \Delta I(t)$ and $\sigma^{2}$ as above, but for the total intensity $I$ instead of $I_{m}$.

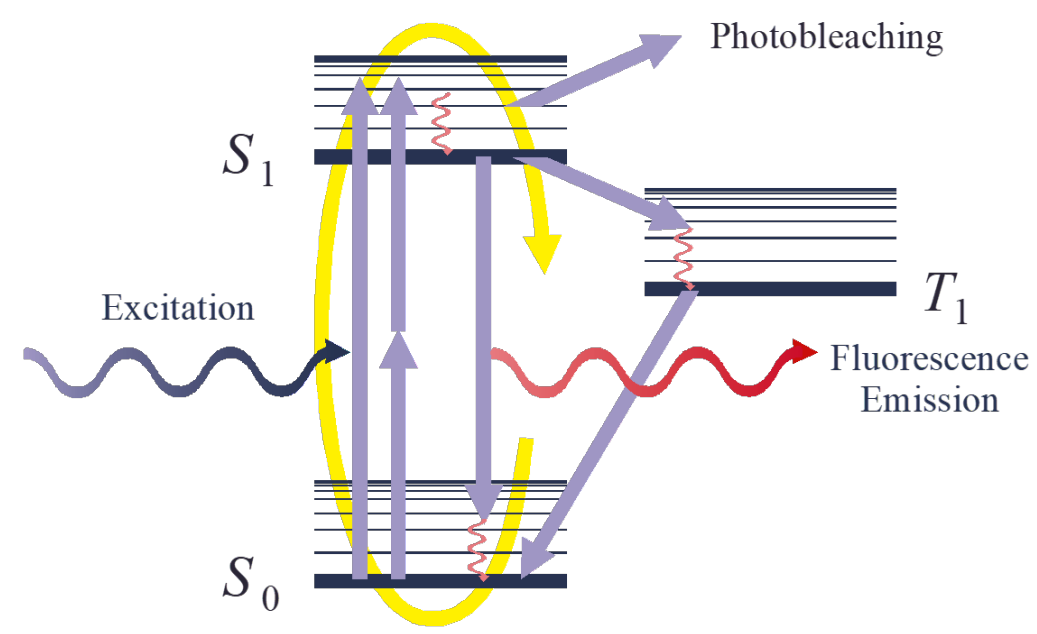

Figure 2.10: The Jablonski diagram is a simplified scheme of the energy levels of a molecule labeled with a fluorophore. On the bottom (lowest energy) is its electronic ground state $S_{0}$. Upon absorption of one or more photons, its energy level gets excited to the first excited state $S_{1}$. Several physical processes (possibly occuring on different time scales) allow the excited molecule to emit energy and return to $S_{0}$. These processes include not only photobleaching (due to which the molecule permanently loses its fluorescent properties), transitions to a long-lived triplet state $T_{1}$ and energy transfer to its surroundings (vibrational, translational or rotational modes, interactions with quenchers), but also the emission of a photon. This photon has a lower energy than the initially absorbed photon because the absorbed energy is partially lost to the mentioned competing mechanisms of energy transfer (depicted in the diagram as small energetic steps around $S_{0}, S_{1}$ and $T_{1}$ respectively). This phenomenon is called the Stokes shift - the wavelength of the fluorescence light is longer than that of the excitation light. Repeated cycling between $S_{0}$ and $S_{1}$ (yellow loop) thus leads to light being emitted from the labeled molecule, which is called fluorescence. Transitions back to the ground state $S_{0}$ after getting excited typically take place on a time scale of several nanoseconds (fluorescence lifetime). This figure was kindly provided to me by Jörg Enderlein. 


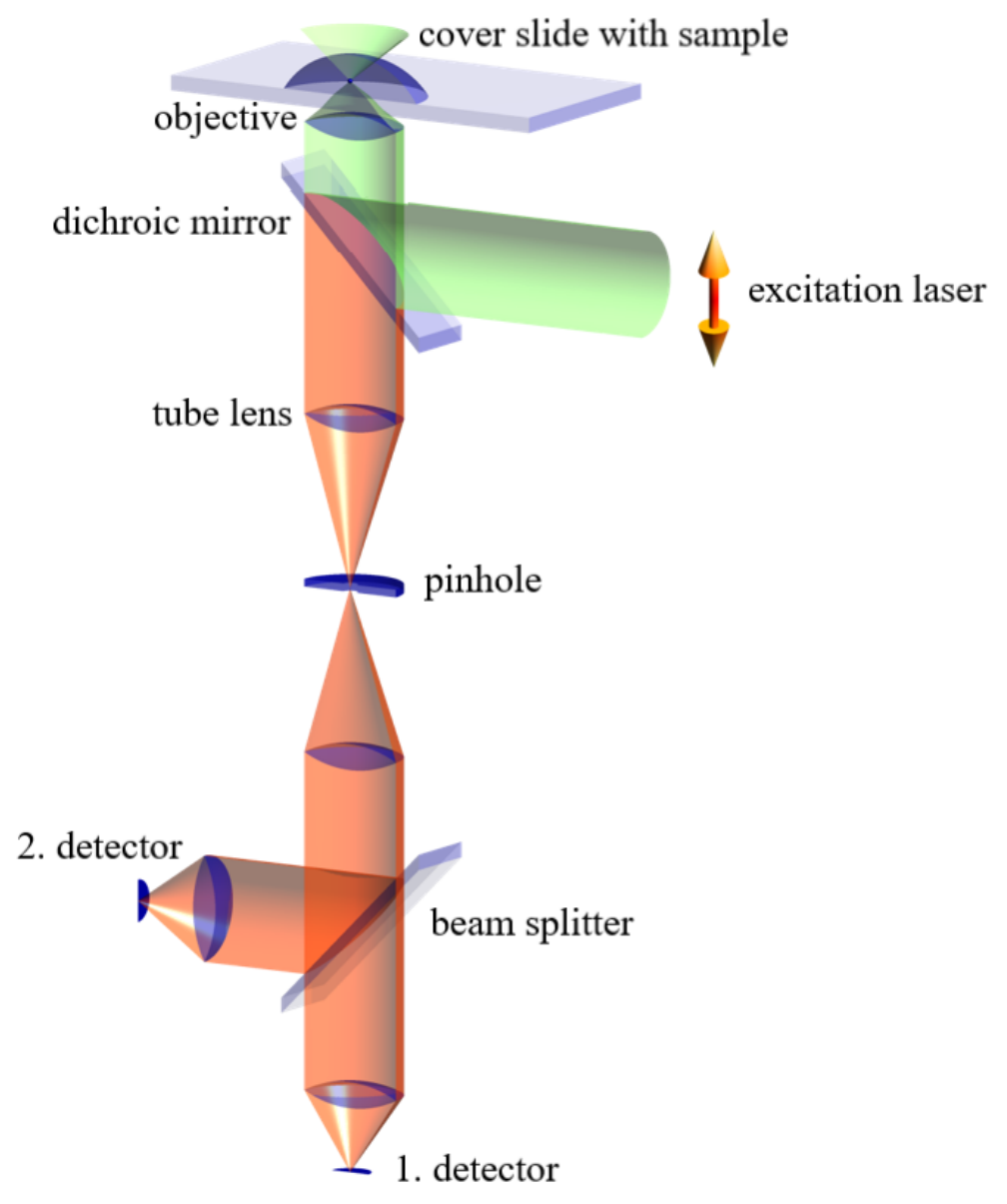

Figure 2.11: The experimental setup for single-molecule spectroscopy. An excitation laser beam (green beam) with wavelength $\lambda_{1}$ is pointed onto a dichroic mirror which reflects light with wavelength $\lambda_{1}$ upwards. Using an objective lens, the laser light is focused into a small excitation volume (on the order of $\mu \mathrm{m}^{3}$ ) in the sample wherein it excites fluorophores. The fluorescence light (red beam) with wavelength $\lambda_{2}>\lambda_{1}$ is emitted isotropically from this volume, and the fraction of it which hits the objective is made parallel by it. The dichroic mirror does not reflect light with wavelengths $\lambda_{2}$, but lets it pass through. The fluorescence signal is then focused by a tube lens such that it passes exactly through a small pinhole. This step filters out the non-parallel light entering the tube lens, as the tube lens does not focus it onto the pinhole. It is crucial in order to preserve the fluorescence signal while subtracting unwanted light from the surroundings. Unwanted light is mainly due to inelastic (Raman) scattering of the laser light with $\lambda \approx \lambda_{2}$, which is thus not reflected by the dichroic mirror. Next the fluorescence signal is split into two equal beams with halved intensity by a beam splitter, which are independently detected by two detectors. Thereby one bypasses the technical limitation that after detecting a photon, a single detector cannot detect another photon for a few hundred nanoseconds (dead-time), and thus the fluorescence signal emitted by the sample can be detected at almost all times. This figure was kindly provided to me by Jörg Enderlein. 
For practical purposes which will soon become clear, let us further define the functions $h$ and $f$ as

$$
h(\tau \mid \Delta t):=\frac{1}{\Delta t} \int_{-\Delta t / 2}^{\Delta t / 2} f\left(\tau+t^{\prime}\right) \mathrm{d} t^{\prime} \approx f(\tau):=\langle I(t) \cdot I(t+\tau)\rangle=\sigma^{2} C(\tau)+\langle I\rangle^{2}
$$

where $\Delta t$ should be small compared to the time scale on which $f(\tau)$ changes for the approximation $f \approx h$ to be adequate. Now we firstly show that $h(\tau \mid \Delta t)$ is the FCS histogram (with bin size $\Delta t$ ) of arrival time differences of all detected photon pairs. Secondly, we show that $C_{m}(\tau)$ can be extracted from $f(\tau)$, and conclude that FCS indeed yields information about the dynamics of single molecules. First:

$$
\begin{aligned}
h(\tau \mid \Delta t) & =\frac{1}{\Delta t} \int_{-\Delta t / 2}^{\Delta t / 2}\left\langle I(t) \cdot I\left(t+\tau+t^{\prime}\right)\right\rangle \mathrm{d} t^{\prime} \\
& =\frac{1}{\Delta t \cdot T} \int_{-\Delta t / 2}^{\Delta t / 2} \int_{0}^{T} I(t) \cdot I\left(t+\tau+t^{\prime}\right) \mathrm{d} t^{\prime} \mathrm{d} t \\
& \stackrel{2.185}{=} \frac{1}{\Delta t \cdot T} \sum_{p, q=1}^{P} \int_{-\Delta t / 2}^{\Delta t / 2} \int_{0}^{T} \delta\left(t-t_{p}\right) \cdot \delta\left(t+\tau+t^{\prime}-t_{q}\right) \mathrm{d} t^{\prime} \mathrm{d} t \\
& \propto \sum_{p, q=1}^{P} \int_{-\Delta t / 2}^{\Delta t / 2} \delta\left(t_{p}-t_{q}+\tau+t^{\prime}\right) \mathrm{d} t^{\prime}
\end{aligned}
$$

The remaining delta function triggers for all photon pairs $(p, q)$ for which $\tau-\Delta t / 2<\left|t_{p}-t_{q}\right|<$ $\tau+\Delta t / 2$. Hence, prefactors aside, we have at hand a histogram of arrival times between photons, centered at $\tau$ with bin size $\Delta t$. Second:

$$
\begin{aligned}
f(\tau) & =\sum_{m, n=1}^{M}\left\langle I_{m}(t) \cdot I_{n}(t+\tau)\right\rangle_{t} \\
& =\sum_{m=1}^{M} \sum_{n \neq m}^{M}\left\langle I_{m}\right\rangle\left\langle I_{n}\right\rangle+\sum_{m=1}^{M}\left\langle I_{m}(t) \cdot I_{m}(t+\tau)\right\rangle_{t} \\
& =M \cdot(M-1) \cdot\left\langle I_{m}\right\rangle^{2}+M \cdot\left\langle I_{m}(t) \cdot I_{m}(t+\tau)\right\rangle_{t} \\
& =M \cdot(M-1) \cdot\left\langle I_{m}\right\rangle^{2}+M \cdot\left(\sigma_{m}^{2} C_{m}(\tau)+\left\langle I_{m}\right\rangle^{2}\right) \\
& =M^{2} \cdot\left\langle I_{m}\right\rangle^{2}+M \cdot \sigma_{m}^{2} C_{m}(\tau)
\end{aligned}
$$

where we have assumed that the intensities coming from different molecules are uncorrelated such that their average product factorizes, and that the different molecules' fluorescence is statistically identical. The recipe is thus to measure photon arrival times, calculate the histogram $h(\tau \mid \Delta t) \approx f(\tau)$ for an appropriately small value of $\Delta t$ (a too small value yields too few photon pairs in each bin, and thus a very noisy histogram) and then compare

$$
C_{m}(\tau) \approx \frac{h(\tau \mid \Delta t)-h(\infty \mid \Delta t)}{h(0 \mid \Delta t)-h(\infty \mid \Delta t)} \stackrel{\tau \rightarrow \infty}{\longrightarrow} 0
$$

with simulation results. In experimental practice, one does not calculate a histogram with equidistant bin size, but chooses exponentially growing (equidistant on a logarithmic time axis) bin sizes because $C(\tau)$ varies more slowly for later times, and more photons contribute to later bins this way. 


\subsubsection{Photo-induced electron transfer (PET)}
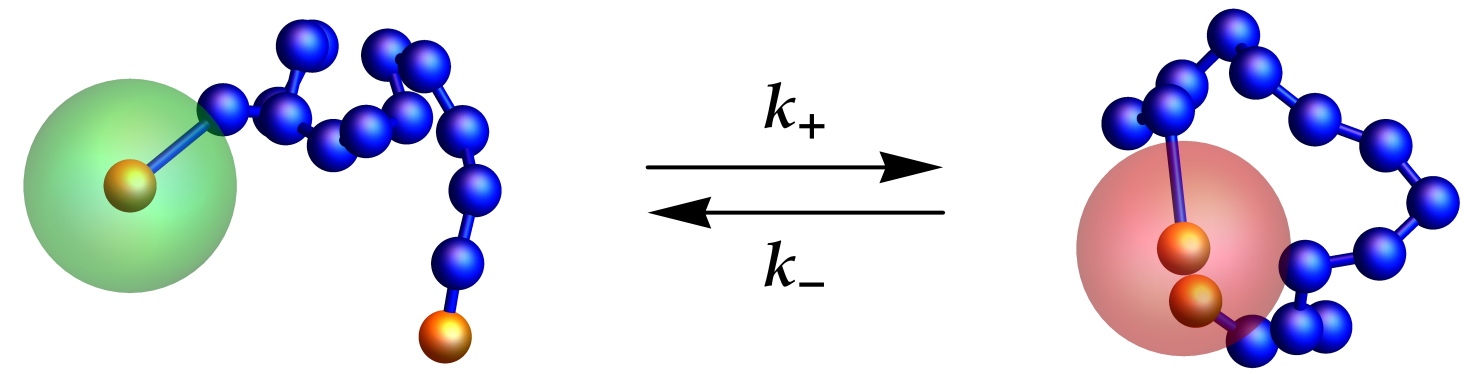

Figure 2.12: Schematic representation of a single molecule labeled with the fluorescent dye Atto655 (orange bead surrounded by red/green sphere) and the quencher tryptophan (lone orange bead). When the dye and quencher are spatially separated (open state, left), fluorescence is turned on as indicated by the sphere around the dye being green. The sphere turns red when the distance between dye and quencher deceeds a certain threshold (its radius), indicating that fluorescence is turned off (closed state, right). Transitions between the open and closed state stochastically occur with rates $k_{+}$and $k_{-}$respectively.

Photo-induced electron transfer [117] (PET) is a physical effect that can be combined with FCS. For our purposes, PET is an interaction between the excited fluorophore Atto655 and the amino acid tryptophan (W), which are both part of (or bound to) the protein under investigation. In the experiments, the labeled proteins diffuse freely through water, thus entering and leaving the excitation focus randomly. At the same time, each molecule constantly undergoes conformational dynamics. When the distance between Atto655 and tryptophan is sufficiently low $(\approx 0.5 \mathrm{~nm}$ or lower), they "stick together" via a $\pi$-stacking interaction of their aromatic ring structures, forming a complex for tens of nanoseconds before the molecule opens up again. In this "closed state", Atto655 transfers energy from its excited state to tryptophan via electron transfer, and afterwards the complex non-radiatively recombines the charges to relax back to the ground state (see reference [8] and references therein). This transfer occurs on a time scale of femto- to picoseconds $[118,119]$, which is significantly faster than the decay to the fluorophore's unexcited ground state via fluorescence, which typically takes several nanoseconds. The consequence of this separation of time scales is that whenever Atto655 and tryptophan are in close proximity, the fluorescence signal emitted by Atto655 is effectively quenched: The molecule turns dark. From an experimentalist's perspective there are thus only two relevant mesostates of the polymer chain - it can be open and bright, or it can be closed and dark (see figure 2.12).

Two-state model Due to this circumstance, let us change our perspective from the chain's full conformational dynamics to mere transitions from the open to the closed state and vice versa. This perspective can be most easily described by a two-state model which is in the open state with probability $p^{\text {open }}$ and in the closed state with probability $p^{\text {closed }}$. 
Given that the molecule is in the open state, we assume that transitions to the closed state occur with a rate $k_{+}$(contact rate or loop formation rate) and in the other direction with a rate $k_{-}$. Using $p^{\text {open }}+p^{\text {closed }}=1$, the time evolution of $p^{\text {open }}$ is thus described by the differential equation

$$
\mathrm{d}_{t} p^{\text {open }}=-k_{+} p^{\text {open }}+k_{-} p^{\text {closed }}=-\left(k_{+}+k_{-}\right) p^{\text {open }}+k_{-}
$$

whose solution, assuming $p^{\text {open }}(0)=1$, is given by

$$
p^{\text {open }}(t)=\frac{k_{-}}{k_{+}+k_{-}}+\frac{k_{+}}{k_{+}+k_{-}} \exp \left(-\frac{t}{\left(k_{+}+k_{-}\right)^{-1}}\right) \quad .
$$

Setting the intensity $I_{m}$ of molecule $m$ to $I_{0}$ in the open state and to 0 in the closed state, it follows that

$$
\left\langle I_{m}(0) \cdot I_{m}(t)\right\rangle=\left\langle I_{0} \cdot I_{m}(t)\right\rangle=I_{0}^{2} \cdot p^{\text {open }}(t)+I_{0} \cdot 0 \cdot p^{\text {closed }}(t)=I_{0}^{2} \cdot p^{\text {open }}(t) \quad .
$$

The initial condition $I_{m}(0)=I_{0}$ reflects the experimental situation at hand: Detecting a photon pair from molecule $m$ implies that at the arrival times of both photon, the molecule was fluorescent. Hence its contribution to the functional form of the FCS histogram can be interpreted as the conditional probability that the molecule is fluorescent at time $\tau$, given that it was fluorescent at time 0. Equation 2.192 confirms this interpretation because, due to its initial condition, $p^{\text {open }}(t)$ is precisely that conditional probability. Note that, at time 0 , the molecule is somewhere in the open state - it has not necessarily just left the closed state!

In practice, there are dynamic effects present in the labeled proteins other than only the openingclosing dynamics which make their intensity time-dependent - for instance their translational diffusion out of the excitation focus where the fluorescence intensity vanishes. Since this diffusion is much slower than the protein's conformational dynamics, the probabilities of being (i) opened or closed and (ii) inside and outside of the excitation focus factorize, leading to an extra factor appearing in equation 2.192. This factor is typically approximated with the term $\left(1+t / t_{D}\right)^{-1}$, where the fit parameter $t_{D}$ is the time scale of the molecules' translational diffusion through the excitation focus. In practice, the normalized experimental histogram (see equation 2.189) is thus fitted with the function

$$
C_{m}(t) \propto\left[1+\frac{k_{+}}{k_{-}} \exp \left(-\frac{t}{\left(k_{+}+k_{-}\right)^{-1}}\right)\right]\left(1+\frac{t}{t_{D}}\right)^{-1} .
$$

A set of measured PET-FCS correlation curves is shown in figure 5.7 on page 116. It turns out that $1 / k_{+}$and $1 / k_{-}$are both on the order of tens to hundreds of nanoseconds, whereas $t_{D}$ is on the order of milliseconds. We remark that under equilibrium conditions $(t \rightarrow \infty)$

$$
\frac{p^{\text {open }}}{p^{\text {closed }}}=\frac{k_{-}}{k_{+}}=\exp \left(-\frac{F^{\text {open }}-F^{\text {closed }}}{T}\right)
$$

such that the free energy difference between the open and closed states is given by

$$
\Delta F=T \ln \frac{k_{+}}{k_{-}} \quad \text {. }
$$


Contact radius value As of now, it is unclear exactly how close the fluorophore and the quencher have to get in order to form a non-fluorescent complex. This question has been addressed in literature [13, 117, 120], and the best value available specifically for Atto655 and tryptophan [117] is a critical contact radius of

$$
R_{\mathrm{C}}=5.4 \pm 0.2 \AA
$$

This value has been determined by comparing (a) the experimentally measured ratio of fluorescent Atto655 molecules in a solution with tryptophan with (b) the probability distribution of the distance between Atto655 and tryptophan in solution extracted from an all-atom (MD) simulation. It should be noted that both the experimental as well as the simulated systems in that study include an interaction energy between Atto655 and tryptophan which our idealized beads model (see next chapter) does not include. Furthermore, it is unclear how the critical contact radius above (value for the minimum distance between any of their respective atoms) translates into the proper distance between bead centers (vertices of our discrete chain). We must therefore bear in mind that the proper critical contact radius in any bead model is only known approximately.

\subsubsection{Dynamic metal-induced energy transfer (dynaMIET)}

Like PET, metal-induced energy transfer (MIET) is an effect that can be combined with FCS in order to gain insight into the dynamics of individual molecules. Doing so leads to a method called dynamic metal-induced energy transfer (dynaMIET). Whereas in PET, the fluorescence intensity is essentially a step function of the distance between the fluorophore and the quencher, in dynaMIET the intensity is a smooth, continuous function of the distance of the fluorophore from a metal surface (see figure 2.14). The experimental sample setup which utilizes this effect is the following (see figure 2.13): The molecule of interest is immobilized by binding one if its chain ends to a surface. The other (free) chain end is labeled with the fluorophore which undergoes Brownian motion, and thus its distance from the surface is a stochastic function of time (see figure 5.22, page 139).

The physical reason for the MIET effect is that there is a rate of energy transfer from the fluorophore's excited state to the metal surface. The strength of this coupling can be calculated within the framework of classical electrodynamics: Maxwell's equations are solved in the presence of a surface and the boundary conditions it imposes on the electric and magnetic field [121-123]. Integrating the Poynting vector around the fluorophore then delivers the total energy flux emitted by the fluorophore (a dipole), and it turns out that this integral - among other parameters which classify the experimental setting (wavelength, various refractive indices, etc.) - depends on the distance of the fluorophore from the surface. The fluorescence intensity is reduced due to the energy transfer to the surface, and the resulting intensity-over-distance curve is shown in 2.14 for one particular set of experimental parameters. 


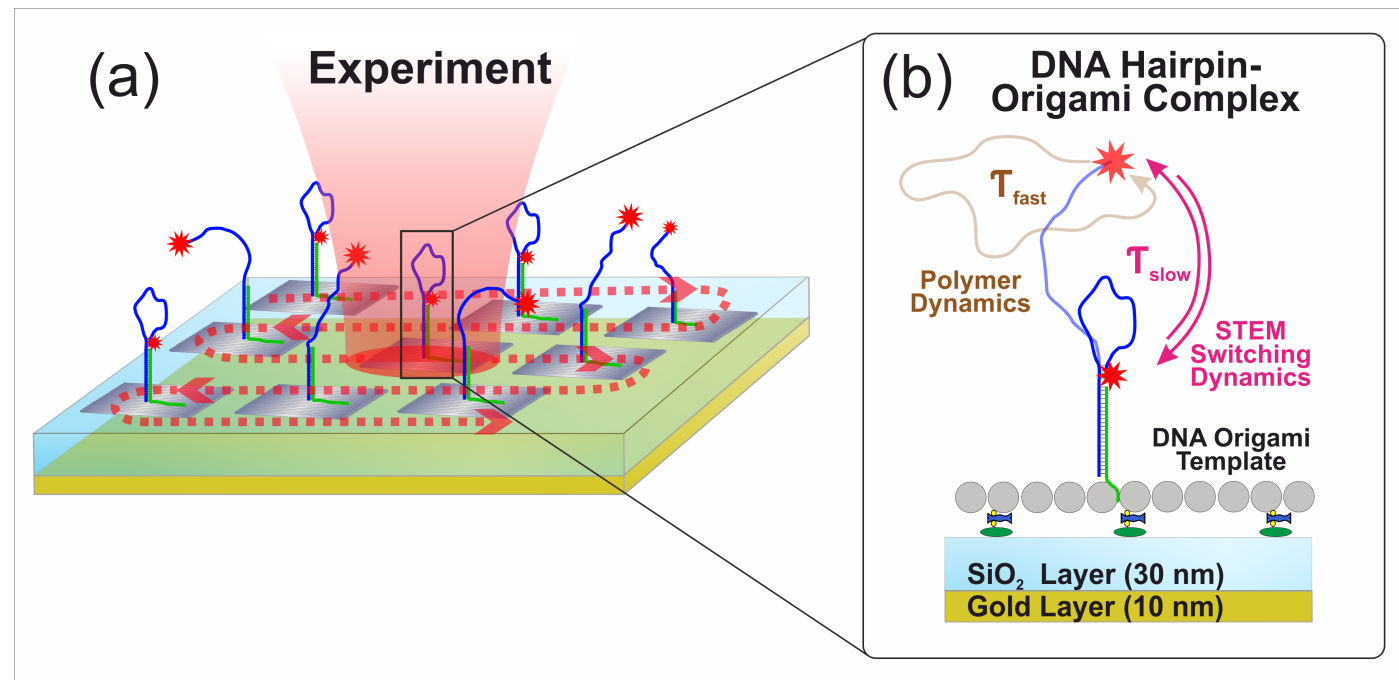

Figure 2.13: Sketch of a typical sample used in dynaMIET experiments. A cover slip is coated with several layers on top of it, the thicknesses of which are in the order of (tens of) nanometers. One of these layers is a metal, gold in this case, which is necessary for the MIET effect to take place. On top of the layers are strands of double-stranded DNA (dsDNA), to which the molecules of interest, single-stranded DNA (ssDNA) in this example, are bound. These molecules are surrounded by water, and are each labeled with a fluorophore at their free end. They undergo Brownian motion, making the dye's intensity time-dependent. In the setup shown here, the end of the ssDNA can bind and unbind to the end of the dsDNA, which occurs on a slower time scale than the free polymer dynamics. In practice, the entire cover slip is covered with (ideally) identical molecules, and the sample is scanned by the excitation focus (dashed red track), in order to overcome the limitation which photobleaching imposes on the number of photons collectable from a single fluorophore. This figure was kindly provided to me by Roman Tsukanov.

Shape of intensity autocorrelation An analytical form for the intensity autocorrelation function in dynaMIET is not available. That is because (i) the polymer dynamics are highdimensional and nonlinear, (ii) only a projection (z-position of chain end) is the relevant observable and (iii) $I(z)$ is also slightly nonlinear. Nevertheless, a simple educated guess can be made assuming Gaussian statistics of the fluorophore's z-position due to the central limit theorem, and further neglecting that the z-dynamics may be highly non-Markovian, one is left with onedimensional diffusion in a harmonic potential. That is because the physical potential associated with a Gaussian probability distribution is quadratic due to the Boltzmann factor in thermal equilibrium. It can be shown that the autocorrelation function of such a process (called the Ornstein-Uhlenbeck process) is a mono-exponential decay [1]. As a first guess, autocorrelation curves from dynaMIET experiments or simulations can thus be fitted with mono-exponentials, and the resulting fit quality be compared with the fit result using a sum of exponential functions. 


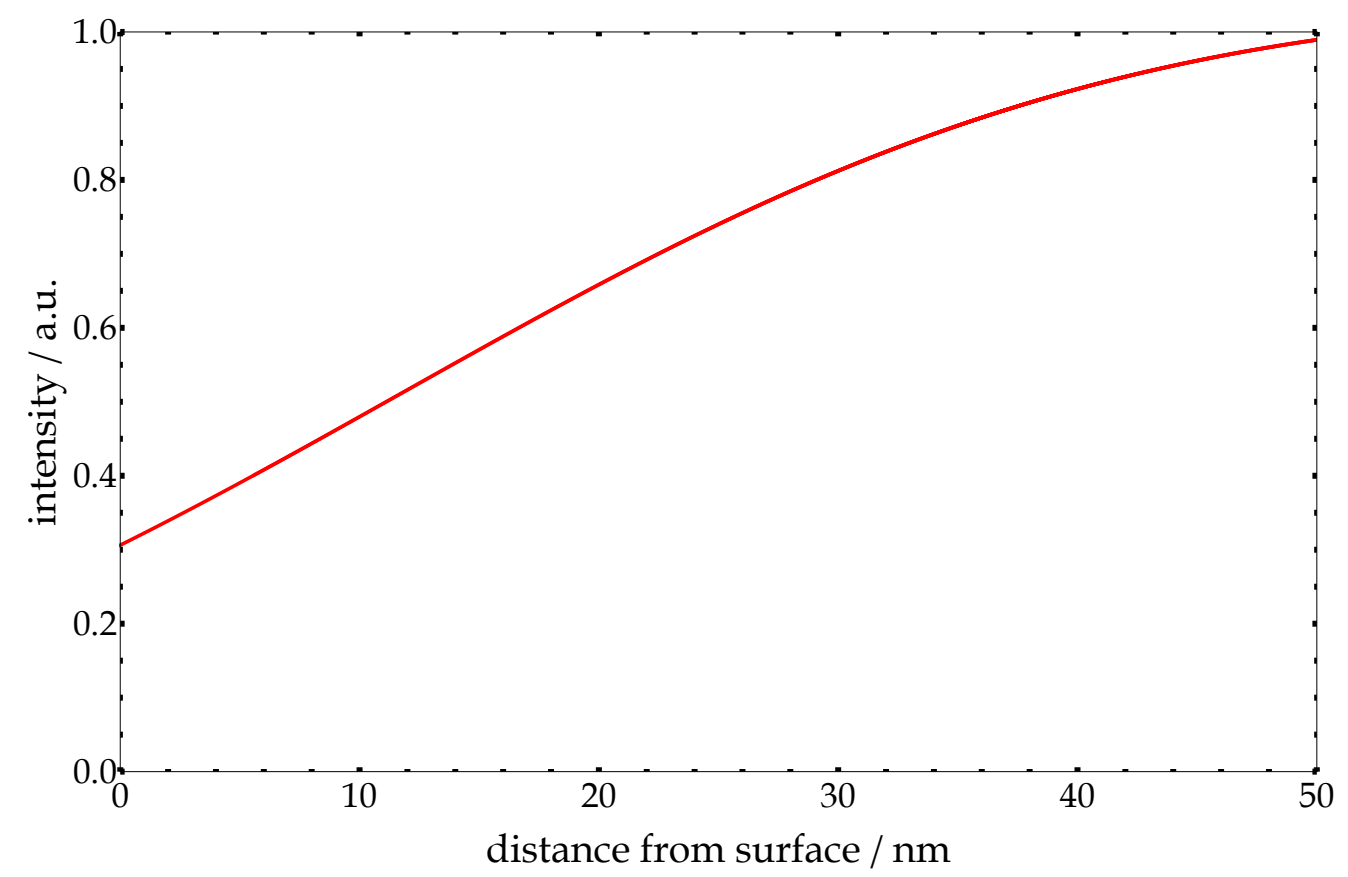

Figure 2.14: The theoretically predicted intensity of a fluorophore as a function of its distance from the topmost layer of the cover slip (see figure 2.13). The fluorophore is typically at most $20-30 \mathrm{~nm}$ away from the surface, thus making the intensity curve virtually linear. This curve was calculated with a Matlab-script provided by Daja Ruhlandt. 


\subsubsection{Dual-focus fluorescence correlation spectroscopy (2fFCS)}

Dual-focus fluorescence correlation spectroscopy (2fFCS) is an experimental method for measuring the diffusion coefficient (or equivalently the hydrodynamic radius) of single molecules [87]. The experimental setup required for 2fFCS is slightly different from that used for PET-FCS and dynaMIET, and is shown in figure 2.15 .

The idea behind 2fFCS is the following: Instead of autocorrelating the photon-arrival times from molecules in a single excitation volume in the sample, one uses two excitation volumes which are very close to each other. The arrival times of photons from the two foci are then cross-correlated (only photon pairs coming from different foci contribute to the FCS histogram) and histogrammed as a function of their time delay $\tau$. Analogous to regular FCS, this histogram yields information about the probability that a single molecule has been in one focus at time $\tau=0$ and then in the other focus at time $\tau$. The cross-correlation (histogram) depends on a number of experimental parameters such as the shapes of the foci and the distance between them (see reference [87] for details), but also on the molecules' diffusion coefficient which is thus infered as a fit parameter. In practice, the hydrodynamic radius $R_{H}^{\text {Atto655 }}$ of the fluorophore Atto655 is well-known in literature, and is thus used to determine the molecule's hydrodynamic radius $R_{H}$ via the StokesEinstein relation (see equation 2.131, page 53):

$$
R_{H}=R_{H}^{\text {Atto655 }} \frac{D}{D^{\text {Atto655 }}}
$$

Systematic biases in measuring diffusion coefficients or hydrodynamic radii are thus reduced because $D$ and $D^{\text {Atto655 }}$ are measured independently. 


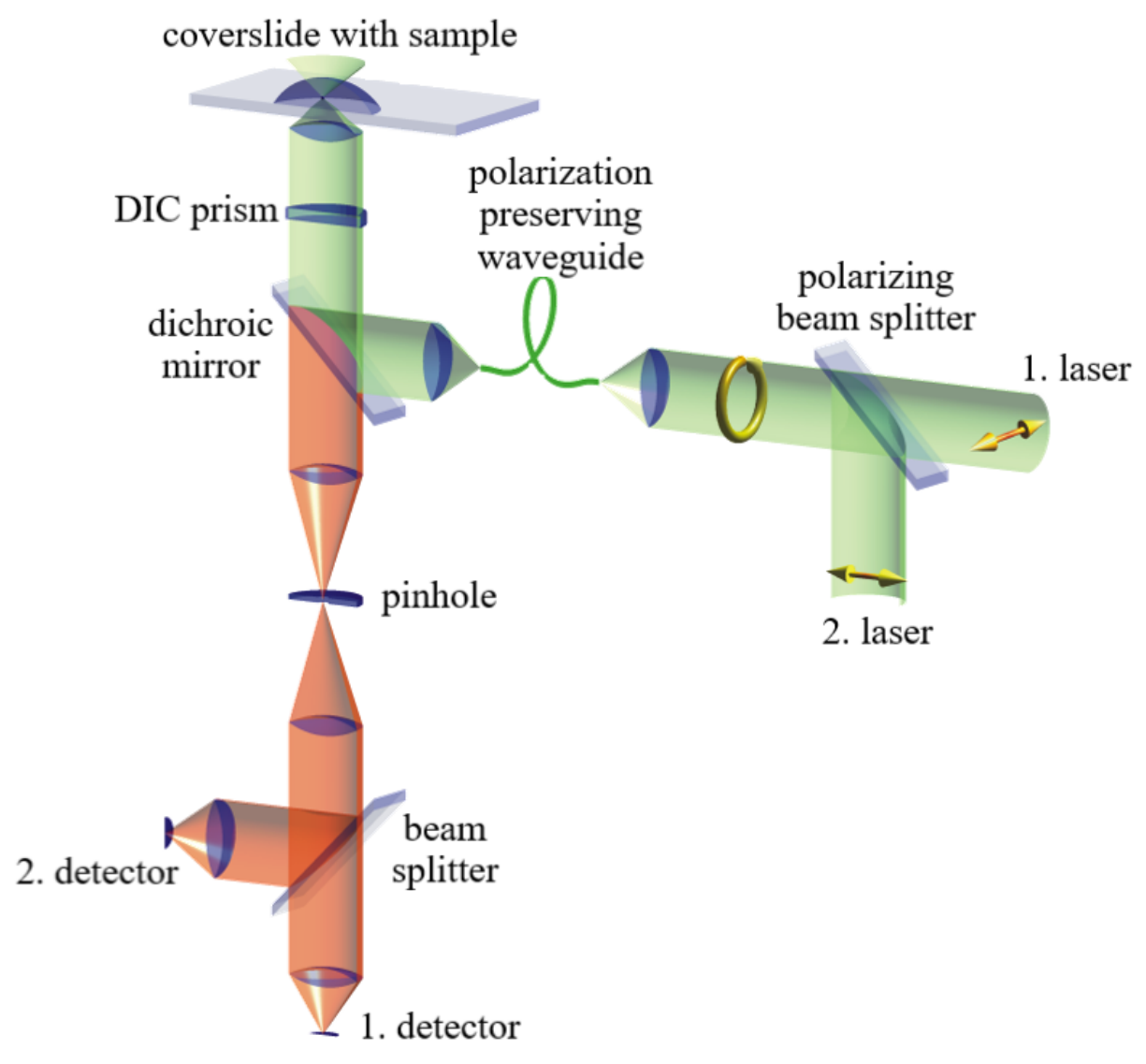

Figure 2.15: The experimental setup used for dual-focus fluorescence correlation spectroscopy (2fFCS). This setup is similar to the FCS setup explained in figure 2.11. The differences are the following: Instead of only one excitation laser, two lasers are used. Their beams and wavelengths coincide, but they are polarized in perpendicular directions. Based on their polarizations, a differential interference contrast (DIC) prism slightly separates the two beams before entering the objective. As a result, the objective lens focuses them into two separate excitation foci in the sample. These foci are spatially separated, but close enough such that the fluorescence light from both foci passes through the pinhole. The two excitation lasers are pulsed and send out light alternatingly. The time between pulses is on the order of tens of nanoseconds. Thereby, when a photon is detected in any detector, it can be inferred which laser pulse is the most recent, and therefore it is also known which laser the pulse belongs to and from which excitation volume in the sample the photon was emitted. This figure was kindly provided to me by Jörg Enderlein and published in [87]. 


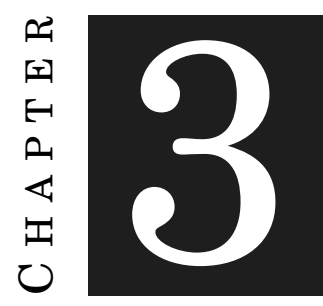

\section{THE POLYMER MODEL}

In this chapter, we define our polymer model, present its equation of motion and discuss its parameters. Any real polymer embedded in a thermal fluid has the following properties:

1. It undergoes thermal fluctuations.

2. It cannot cross through itself.

3. Neighboring tangent vectors have finite angular correlation.

4. Its subunits stay connected along the chain.

5. Its subunits experience solvent friction and hydrodynamic interactions.

With the preparations of the previous chapter at hand, it is straightforward to define our polymer model which fulfills all the criteria above: We embed a discrete elastic rod (section 2.3.2) in an overdamped fluid (section 2.1.5) which is in thermal equilibrium (section 2.2.6). Its bending energy $E$ quantifies elastic forces and results in a finite persistence length (criterion 3). A spherical bead with hydrodynamic radius $a$ is associated with each of the chain's vertices, including its end points. The hydrodynamic diffusion tensor $\mathbf{D}$ within the Rotne-Prager approximation quantifies solvent friction via its diagonal blocks $\mathbf{D}_{i i}$, and also hydrodynamic interactions via its off-diagonal blocks $\mathbf{D}_{i j}$ (criterion 5). The beads' dynamics is described by the Langevin equation (criterion 1) underlying inextensibility constraints: the distances between neighboring beads are fixed to the bond length $d$ (criterion 4). In order to fulfill criterion 2 , we introduce an excluded volume radius $R_{\mathrm{EV}}$ which is associated with each bead (hard sphere): any two beads $\vec{r}_{i}$ and $\vec{r}_{j}$ are prohibited from overlapping in the sense that $\left|\vec{r}_{i}-\vec{r}_{j}\right|<2 R_{\mathrm{EV}}$. 
The two bead radii $a$ and $R_{\mathrm{EV}}$ must not necessarily coincide: One is related to geometric constraints on protein motion, while the other is related to hydrodynamic coupling and drag, taking into account the hydration shell of tightly bound water molecules around an amino acid.

To furthermore mimic the experimental situation at hand more accurately and make use of all available information, we take the fluorophore with which the real molecules are labeled into account. This is easily achieved within our model by allowing the chain's first bead to possess a given hydrodynamic radius $a_{1}$ and the first edge (bond) to have a given length $d_{1}$, both of which may reflect the fluorophore's properties rather than the protein's. A sketch of our polymer model can be found in figure 3.1.

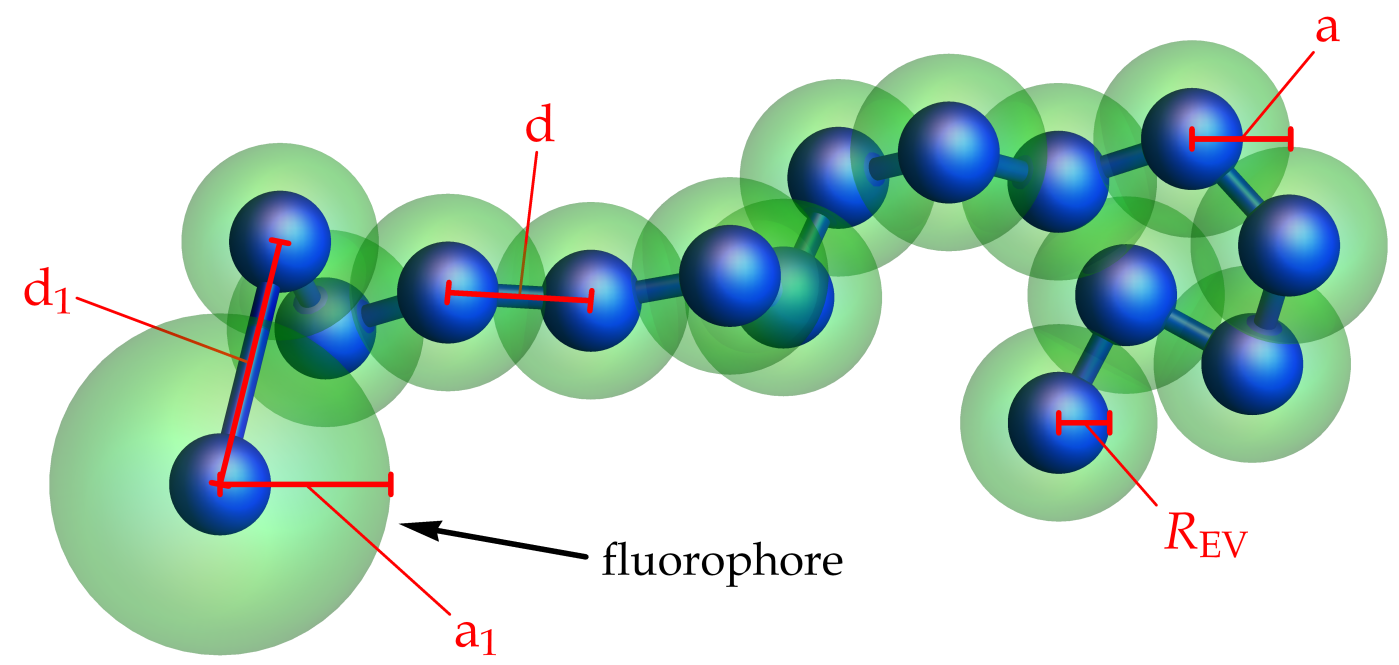

Figure 3.1: A sketch of one particular conformation of our polymer model. It consists of $N$ spherical beads. The blue spheres show their excluded volume radius $R_{\mathrm{EV}}$, and the green spheres show their hydrodynamic radius $a$. The bond lengths $d$ (rods) in the model are fixed and are only shown for visual purposes. The first bead has its own hydrodynamic radius $a_{1}$ and bond length $d_{1}$, which enable it to represent the fluorophore rather than e.g. an amino acid. Parameters which are not shown in this sketch are the chain's bending stiffness $\sigma$ and the fluid's temperature $T$ and viscosity $\eta$.

From the perspective of polymer theory, our model can be classified as follows: We have at hand an overdamped, semiflexible bead-rod chain with excluded volume effects, hydrodynamic interactions and an attached fluorophore. 
Equations of motion As we established in the previous chapter, the constrained (section 2.2.6, page 48) Langevin equation (section 2.2.5, page 41) for the polymer model described above reads

$$
\mathrm{d} \vec{r}_{i}=T^{-1} \sum_{j=1}^{N} \mathbf{D}_{i j} \cdot\left[-\vec{\nabla}_{j}\left(E_{\mathrm{EV}}-T S_{C}\right)+\lambda_{i+1} \vec{t}_{i+1}-\lambda_{i} \vec{t}_{i}\right] \mathrm{d} t+\sqrt{2} \sum_{j=1}^{N} \mathbf{B}_{i j} \cdot \mathrm{d} \vec{W}_{j}
$$

where $i=1, \ldots, N, \mathbf{D}=\mathbf{B} \cdot \mathbf{B}^{T}, \lambda_{0}=\lambda_{N}=0, \vec{t}_{i}=\frac{\vec{r}_{i+1}-\vec{r}_{i}}{\left|\vec{r}_{i+1}-\vec{r}_{i}\right|}$ and

$$
E_{\mathrm{EV}}= \begin{cases}E & \text { if } \min _{i, j}\left|\vec{r}_{i}-\vec{r}_{j}\right|>2 R_{\mathrm{EV}} \\ \infty & \text { otherwise }\end{cases}
$$

The $N-1$ inextensibility constraints (page 50) read

$$
\text { and } \begin{aligned}
\left|\vec{r}_{k+1}-\vec{r}_{k}\right| & =d \\
\left|\vec{r}_{2}-\vec{r}_{1}\right| & =d_{1} \quad .
\end{aligned}
$$

The bending energy $E$ is given by equation 2.158 (page 62) and on page 68 we explained how it can be translated into a persistence length. The constraint-entropy gradient $\vec{\nabla} S_{C}$ is given by equation C.14 (page 181) and the diffusion matrix $\mathbf{D}$ is given by equation 2.29 (page 24). This expression for the diffusion matrix can be simplified in the case of equally sized beads (size $a$ ), with only the first one being differently sized (size $a_{1}$ ). It reads

$$
\boldsymbol{D}_{i j}= \begin{cases}\frac{3}{4} \cdot D_{0} \cdot \frac{a}{r_{i j}} \cdot\left[\left(1+\frac{a_{i}^{2}+a_{j}^{2}}{3 r_{i j}^{2}}\right) \mathbb{1}_{3 \times 3}+\left(1-\frac{a_{i}^{2}+a_{j}^{2}}{r_{i j}^{2}}\right) \frac{\vec{r}_{i j} \vec{r}_{i j}^{T}}{r_{i j}^{2}}\right] & \text { if } \quad a_{i}+a_{j}<r_{i j} \\ D_{0} \cdot\left[\frac{16 r_{i j}^{3}\left(a_{i}+a_{j}\right)-\left(\Delta a^{2}+3 r_{i j}^{2}\right)^{2}}{a_{>} \cdot 32 r_{i j}^{3}} \mathbb{1}_{3 \times 3}+\frac{3\left(\Delta a^{2}-r_{i j}^{2}\right)^{2}}{a_{>} \cdot 32 r_{i j}^{3}} \frac{\vec{r}_{i j} \vec{r}_{i j}^{T}}{r_{i j}^{2}}\right] & \text { if } \quad \Delta a<r_{i j} \leq a_{i}+a_{j} \\ D_{0} \cdot \frac{a_{1}}{a} \mathbb{1}_{3 \times 3} & \text { if } \quad r_{i j} \leq \Delta a\end{cases}
$$

where $D_{0}=\frac{T}{6 \pi \eta a}$ is the diffusion coefficient of a single bead, $r_{i j}=\left|\vec{r}_{i}-\vec{r}_{j}\right|, a_{>}=\max \left(a_{i}, a_{j}\right)$ and $\Delta a=\left|a_{i}-a_{j}\right|$. For $i \neq 1 \neq j, \Delta a$ can immediately be set to zero and $a_{i}+a_{j}$ to $2 a$. Furthermore, we have already utilized the fact that $\mathbf{D}$ is divergence-free.

Parameters The parameters included in our model are summarized in table 3.1. Of these nine parameters, only two are free fit parameters: The number of beads $N$ can be freely chosen without altering the characteristic properties of the polymer model itself, and is dictated by the application at hand: Later on, one bead represents one amino acid, and the number of amino acids in experimentally studied proteins is known. The same holds true for the bond length $d$ : The distance between two consecutive amino acids in a protein (i.e. the distance between $C_{\alpha}$ atoms) equals $d=3.8 \AA$. 


\begin{tabular}{c|c}
\hline symbol & name \\
\hline$N$ & number of beads \\
$a$ & hydrodyn. bead radius \\
$a_{1}$ & hydrodyn. radius of first bead \\
$d$ & bond length \\
$d_{1}$ & length of first bond \\
$\sigma$ & bending stiffness \\
$\eta$ & fluid viscosity \\
$T$ & temperature \\
$R_{\mathrm{EV}}$ & excluded volume radius \\
\hline
\end{tabular}

Table 3.1: The nine parameters appearing in our polymer model.

Moreover, the two parameters $a_{1}$ and $d_{1}$ have only been introduced for the first bead to represent the fluorophore with which experimentally studied proteins are labeled. Fortunately, the relevant properties of the fluorophore used for this thesis (Atto655) are known: $a_{1}=6 \AA$ and $d_{1}=7 \AA$ [124]. The fluid around the polymer (heat bath) determines the two parameters $\eta$ and $T$, which are hence not model parameters but known environmental parameters. Furthermore, the purpose of the excluded volume radius $R_{\mathrm{EV}}$ is to prevent the model chain from crossing through itself. We set it to $R_{\mathrm{EV}}=\sqrt{2} / 4 \cdot d \approx 0.35 \cdot d$, which is the minimum value preventing bond crossings while interfering with the local bending stiffness the least. This value was chosen for this geometric reason alone and is also the value displayed in figure 3.1.

Therefore, only two model parameters remain: The chain's bending stiffness $\sigma$ and one bead's hydrodynamic radius $a$. Both indeed quantify characteristic properties of the molecule itself. We reiterate that (given the other parameters) $\sigma$ alone determines all statistical properties of our polymer model, while $a$ influences only dynamical properties. That is because $\sigma$ enters the Boltzmann distribution, which is independent of the diffusion matrix $\mathbf{D}$ and thus independent of $a$. The influence of $a, \sigma$ and $N$ on the model's dynamics will be investigated in section 5.1 (page 107), and the numerical method to solve the constrained Langevin equation is explained on page 89.

Let us agree upon the following three conventions:

1. If $a_{1}$ and $d_{1}$ are not explicitly specified, they equal $a$ and $d$ respectively.

2. If $R_{\mathrm{EV}}$ is not specified, excluded volume effects are not taken into account such that $R_{\mathrm{EV}}=0$.

3. The bound-end constraint (page 50) only applies if explicitly stated. Otherwise, the chain is swimming freely. 


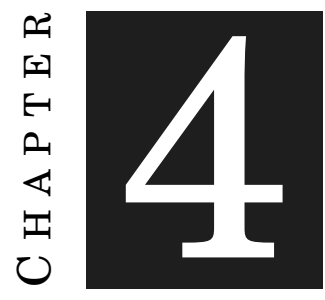

\section{NUMERICAL METHODS}

$\mathrm{I}^{\mathrm{n}}$

$\mathrm{n}$ the previous chapters, the theoretical background for this thesis has been laid and we have arrived at a polymer model whose dynamics is described by the geometrically constrained Langevin equation. In this chapter, we introduce the numerical methods used to (i) generate trajectories from the Langevin equation, (ii) efficiently perform ensemble averages by sampling the Boltzmann distribution, (iii) calculate the hydrodynamic radius and (iv) calculate the contact rate from a model chain's trajectory. Finally, we benchmark these methods by checking that they reproduce known analytical results.

\subsection{Nondimensionalization}

Quantities appearing in physical equations often carry a physical unit: a weight may be measured in kilograms, a time in seconds, and so forth. If we measured lengths in meters, Cartesian coordinates $x$ in the Langevin equation would take on values around $x \approx 10^{-10} \mathrm{~m}$. In order to avoid numerical rounding errors, it is purposeful to have at hand variables which take on values on the order of 1 . In this example, this could easily be achieved by measuring $x$ in nanometers instead. However, not only decimal multiples of SI-units can serve as physical units, and it pays off to make use of the physical system's natural units. For instance, in section 2.1.3 (see page 17) we nondimensionalized the Navier-Stokes equation by manually constructing units from the application at hand: an object embedded in a fluid. The object's length $r_{0}$ and velocity $v_{0}$, and the fluid's viscosity $\eta$ and density $\rho$ enabled us to construct a length scale, a time scale, a pressure scale and a force scale. Consequently the total number of free parameters was reduced, and instead a single "aggregated" parameter emerged naturally: the Reynolds number $\operatorname{Re}=\rho r_{0} v_{0} \eta^{-1}$. 
More formally, physical quantities $y$ are replaced by primed variables such that $y=y^{\prime} \cdot y_{0}$. Here, $y_{0}$ serves as a unit (scale) and $y^{\prime}$ is dimensionless and takes on values on the order of 1 . Numerical computations are then only performed at the level of the primed variables. Afterwards the numerical results are translated back into the physical quantity $y$ by multiplying them with $y_{0}$.

This formal procedure will now be performed for all quantities which possess a physical dimension and appear in the constrained Langevin equation. We choose the temperature $T$ as an energy scale, the bond length $d$ as a length scale, and the diffusion coefficient of a single bead $D_{0}=$ $T /(6 \pi \eta a)$ as a "diffusion" scale. From these three values alone, all other scales can be constructed as follows:

$$
\begin{aligned}
\vec{r} & =d \cdot \vec{r}^{\prime} \\
a & =d \cdot a^{\prime} \\
\mathbf{D} & =D_{0} \cdot \mathbf{D}^{\prime} \\
\vec{\nabla} & =(1 / d) \cdot \vec{\nabla}^{\prime} \\
\sigma & =d \cdot \sigma^{\prime} \\
\Delta s & =d \cdot \Delta s^{\prime} \\
E & =T \cdot E^{\prime}=T \cdot \frac{\sigma^{\prime}}{2} \sum_{k=2}^{N-1} \frac{\left(2 \tan \left(\theta_{k} / 2\right)\right)^{2}}{\Delta s^{\prime}} \\
\lambda & =(T / d) \cdot \lambda^{\prime} \\
t & =\left(d^{2} / D_{0}\right) \cdot t^{\prime} \\
\mathbf{B} & =\sqrt{D_{0}} \cdot \mathbf{B}^{\prime} \\
\mathrm{d} \vec{W} & =\sqrt{t_{0}} \cdot \mathrm{d} \vec{W}^{\prime}
\end{aligned}
$$

Inserting these primed variables into the constrained Langevin equation yields

$$
\mathrm{d} \vec{r}_{i}^{\prime}=\sum_{j=1}^{N} \mathbf{D}_{i j}^{\prime} \cdot\left[-\vec{\nabla}_{j}^{\prime}\left(E^{\prime}-S_{C}\right)+\lambda_{i+1}^{\prime} \vec{t}_{i+1}-\lambda_{i}^{\prime} \vec{t}_{i}\right] \mathrm{d} t^{\prime}+\sqrt{2} \sum_{j=1}^{N} \mathbf{B}_{i j}^{\prime} \cdot \mathrm{d} \vec{W}_{j}^{\prime}
$$

where $\quad 1=\left|\vec{r}_{k+1}^{\prime}-\vec{r}_{k}^{\prime}\right|$

and $\quad d_{1}^{\prime}=\left|\vec{r}_{2}^{\prime}-\vec{r}_{1}^{\prime}\right|$.

Note that the dimensional and nondimensional equations of motion evidently differ only in that the first explicitly includes the temperature $T$, which is not true. In the most general case, the dimensional equations of motion include a total of 9 physical parameters whose values must be specified to find a solution (see also table 3.1, page 84): $d, d_{1}, a, a_{1}, \sigma, T, \eta, N$, and $R_{\mathrm{EV}}$. The nondimensional equations require only $6: d_{1}^{\prime}, a^{\prime}, a_{1}^{\prime}, \sigma^{\prime}, N$ and $R_{\mathrm{EV}}^{\prime}$. As the Buckingham- $\Pi$ theorem dictates [125], this difference of 3 is no coincidence but results from making use of 3 physical scales to nondimensionalize our system. 
The consequence is that dimensional parameter choices which lead to identical values for the non-dimensional parameters can be treated in a single numerical simulations. To translate a simulated, non-dimensional trajectory $\vec{r}^{\prime}\left(t^{\prime}\right)$ back into physical units, we must only specify the length scale $d$ and the time scale

$$
t_{0}=\frac{d^{2}}{D_{0}}=\frac{d^{3} 6 \pi \eta a^{\prime}}{T}
$$

To do so, the "missing parameters" $d, \eta$ and $T$ are required after all. Let us make two remarks. Firstly, since $d^{2}=6 D_{0}\left(t_{0} / 6\right)$, the chosen time scale $t_{0}$ can be interpreted as 6 times the time during which a single bead typically diffuses one bond length. Hence, it is a relevant duration for the local movement of the chain's vertices in that it does not depend on the chain length $N$. It is hence well-suited to simulate loop formation events for different chain lengths with comparable accuracy. Secondly, the hydrodynamic radius $R_{H}$ is a length and is therefore nondimensionalized by dividing it with the bond length $d$; and the contact rate $k_{+}$has the physical unit $1 /$ time and is thus nondimensionalized by multiplying it with $t_{0}$.

Hereafter, we will only deal with the dimensionless quantities and omit the primes for readability. In particular, times and distances will be denoted in units of $t_{0}$ and $d$ unless they are explicitly compared with experimental values.

\subsection{Constraints}

The general case of both inextensibility as well as bound-end constraints being present at the same time will now be discussed. The special case of an inextensible chain which is not bound but freely swimming follows immediately from the general case, which will be demonstrated by the end of this section. The $N$ constraints read

$$
\begin{aligned}
\left|\vec{r}_{1}\right| & =\text { const. } \\
\text { and } \quad\left|\vec{r}_{\mu+1}-\vec{r}_{\mu}\right| & =\text { const. }
\end{aligned}
$$

where $\mu=1, \ldots, N-1$ and $\mu=0$ refers to the first line. To combine them with the Langevin equation, we differentiate them with respect to time, which yields

$$
\text { and } \quad \begin{aligned}
\vec{t}_{0} \cdot \mathrm{d} \vec{r}_{1} & =0 \\
\vec{t}_{\mu} \cdot\left(\mathrm{d} \vec{r}_{\mu+1}-\mathrm{d} \vec{r}_{\mu}\right) & =0
\end{aligned}
$$

where $\mu=1, \ldots, N-1$ and $\vec{t}_{0}=\vec{r}_{1} /\left|\vec{r}_{1}\right|$ as before. According to the constrained Langevin equation, the increments $\mathrm{d} \vec{r}_{i}$ depend linearly on the Lagrangian multipliers $\lambda_{\mu}$. This is fortunate because it means that the differentiated constraints above can be written as a set of linear equations for the Lagrangian multipliers $\vec{\lambda}:=\left(\lambda_{0}, \lambda_{1} \ldots, \lambda_{N-1}\right)^{T}$, which reads

$$
\mathbf{M} \cdot \vec{\lambda}=\vec{v} \quad .
$$


Here, $\mathbf{M}$ is an $N \times N$-matrix and $\vec{v}$ is an $N$-vector. Their entries (whose indices start counting at zero) are derived in appendix $\mathrm{E}$ and read

$$
\begin{aligned}
v_{0} & =-\sum_{j=1}^{N} \vec{t}_{0}^{T} \cdot \mathbf{D}_{1 j} \cdot \vec{F}_{j}^{\mathrm{uc}} \\
v_{\mu} & =-\sum_{j=1}^{N} \vec{t}_{\mu}^{T} \cdot \mathbf{H}_{\mu j} \cdot \vec{F}_{j}^{\mathrm{uc}} \\
M_{00} & =-\vec{t}_{0}^{T} \cdot \mathbf{D}_{11} \cdot \vec{t}_{0} \\
M_{0 v} & =\vec{t}_{0}^{T} \cdot \mathbf{G}_{1 v} \cdot \vec{t}_{v} \\
M_{\mu 0} & =-\vec{t}_{\mu}^{T} \cdot \mathbf{H}_{\mu, 1} \cdot \vec{t}_{0} \\
\text { and } \quad M_{\mu v} & =\vec{t}_{\mu}^{T} \cdot \mathbf{C}_{\mu v} \cdot \vec{t}_{v}
\end{aligned}
$$

where $\mu, v=1, \ldots, N-1$ and the following $3 \times 3$-matrices were defined:

$$
\begin{array}{rlll}
\mathbf{G}_{p q}:=\mathbf{D}_{p q}-\mathbf{D}_{p, q+1}, & & p=1, \ldots, N, & q=1, \ldots, N-1 \\
\mathbf{H}_{p q}:=\mathbf{D}_{p+1, q}-\mathbf{D}_{p q}, & & p=1, \ldots, N-1, & q=1, \ldots, N \\
\mathbf{C}_{p q}:=\mathbf{H}_{p q}-\mathbf{H}_{p, q+1}, & & p=1, \ldots, N-1, & q=1, \ldots, N-1
\end{array}
$$

The unconstrained force $\vec{F}^{\mathrm{uc}}$ is

$$
\vec{F}^{\mathrm{uc}}=-\vec{\nabla}\left(E-S_{C}\right)+\mathbf{D}^{-1} \cdot\left(\vec{\nabla}^{T} \cdot \mathbf{D}\right)+\sqrt{2}\left(\mathbf{B}^{T}\right)^{-1} \mathrm{~d} \vec{W} / \mathrm{d} t \quad
$$

Once these expressions are evaluated, the Lagrangian multipliers are easily identified as

$$
\vec{\lambda}=\mathbf{M}^{-1} \cdot \vec{v}
$$

In each simulation time step, the Lagrangian multipliers must be determined anew and then be inserted into the equations of motion. That is because $\mathbf{M}$ depends on the chain conformation, and because $\vec{v}$ depends on both the chain conformation and the unconstrained forces simultaneously.

In order to treat the case of a freely swimming, inextensible chain, the bound-end constraint can easily be dropped by discarding all terms from equation 4.7 with at least one index which is zero, and by setting $\lambda_{0}=0$. Thus the dimensionality of equation 4.6 is reduced by 1 and the elements of $\mathbf{M}$ and $\vec{v}$ are

$$
\begin{aligned}
v_{\mu} & =-\sum_{j=1}^{N} \vec{t}_{\mu}^{T} \cdot \mathbf{H}_{\mu j} \cdot \vec{F}_{j}^{\mathrm{uc}} \\
\text { and } \quad M_{\mu v} & =\vec{t}_{\mu}^{T} \cdot \mathbf{C}_{\mu v} \cdot \vec{t}_{v}
\end{aligned}
$$

where $\mu, v=1, \ldots, N-1$. 


\subsection{Simulation algorithm}

The simulation algorithm is shown as a flow chart in figure 4.1. It involves a method called Monte-Carlo sampling (MC sampling) which is explained in the section following this one. In order to start a simulation, one needs to specify six dimensionless parameters - the chain length $N$, the bending stiffness $\sigma$, the hydrodynamic bead radius $a$, the excluded volume radius $R_{\mathrm{EV}}$ and the first bead's hydrodynamic radius $a_{1}$ and the first bond length $d_{1}$ (the latter 5 in units of $d$ ). The algorithm itself requires three more parameters - the numerical time step size $\Delta t$, the constraint tolerance $t o l_{1}$ and the material frame tolerance $t o l_{2}$. If applicable, the twisting stiffness and reference curvatures (see equation 2.156, page 61) must also be specified.

Initialization $\mathrm{MC}$ sampling is used to draw $N-2$ bending and azimuthal angles from the Boltzmann distribution. Starting the chain from the origin into $z$-direction, these angles are then used to construct the initial chain. The initial chain is equipped with a Bishop frame which starts at the first chain edge as unit vectors in $x$ and $y$ directions, and is then iteratively constructed using parallel transport. To find the initial material frame, the Bishop frame is rotated by integrated twist angles which are also drawn from their Boltzmann distribution via MC sampling.

Integration step The integration step itself is a midpoint algorithm adapted from reference [67]. It requires that we first calculate the diffusion matrix $\mathbf{D}$ and the stochastic force $\sqrt{2}\left(\mathbf{B}^{T}\right)^{-1} \Delta W / \Delta t$ by performing a Cholesky decomposition of $\mathbf{D}$, and then preproject it. Preprojection means that the force itself is modified using Lagrangian multipliers (as already explained) such that it fulfills the geometric constraints at the level of forces, not velocities. This stochastic force is held fixed throughout the integration step. Then the deterministic forces are evaluated at the chain's state $\vec{r}$ at the beginning of the integration step. Next, the Lagrangian multipliers are determined and the chain's coordinates are updated with a halved time step size $\Delta t / 2$, arriving at the midpoint $\vec{r}^{\text {mid }}$. At the midpoint, the diffusion matrix, deterministic forces and Lagrangian multipliers (but not the stochastic force) are reevaluated and the resulting velocity $\mathrm{d}_{t} \vec{r}$ is used to perform the full time step $\vec{r} \mapsto \vec{r}+\mathrm{d}_{t} \vec{r} \cdot \Delta t$. Throughout this thesis, the value $\Delta t=10^{-3} t_{0}$ is used. Simulated trajectory lengths range from at least $10^{4} t_{0}$ to several $10^{5} t_{0}$, corresponding to $\approx 10^{7}-10^{8}$ or more integration steps. One such trajectory typically takes from several hours up to two days to simulate on a regular desktop PC, using four processor cores such as an Intel Core i5-6600 at $3.30 \mathrm{GHz}$. 


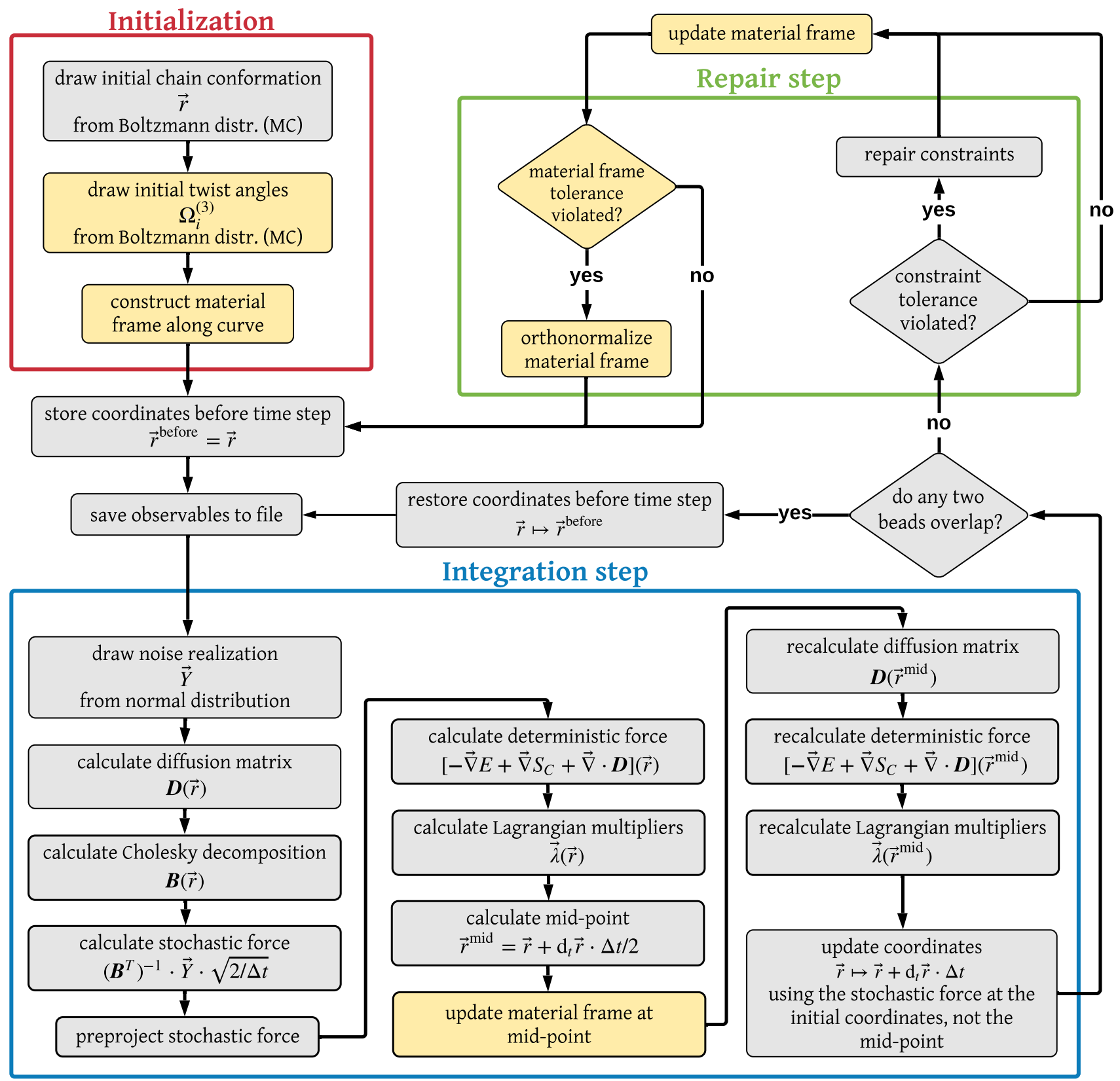

Figure 4.1: This figure shows the numerical algorithm used to generate trajectories from the nondimensional, constrained Langevin equation (equation 4.2, page 86). Starting the algorithm at the top left corner, the initial state of the chain is drawn from the Boltzmann distribution using MC sampling. Afterwards we repeatedly perform integration steps, in between which the observables of interest are stored to an external file. Each integration step that results in any two beads overlapping gets rejected (and the chain before the step restored), and accepted if it does not. After successful integration steps, the geometric constraints and the orthonormality of the material frame are repaired if a predefined tolerance has been violated. For simulations where the material frame is not required, all orange boxes can be omitted. 
Overhead Before each integration step, the chain's current state is stored and observables of interest are saved to an external file. After the integration step, it is checked whether any two beads overlap sterically, i.e. $\min _{i, j}\left|\vec{r}_{i}-\vec{r}_{j}\right|<2 R_{\mathrm{EV}}$. If this is the case, the previously stored state before the integration step is retrieved and the integration step repeated (with a new realization of the random force). In this case the retrieved state gets stored to the external file twice for two consecutive time steps, which is necessary for correct Boltzmann statistics ${ }^{1}$. When a time step is rejected, one may therefore imagine the chain remaining in its state for a time interval of length $\Delta t$. If the integration step was successful, the material frame is updated via parallel transport and it is checked whether either the constraints or the material frame require repair.

Repair step There are two independent repair steps. Firstly, whenever any bond length deviates from 1 (or $d_{1} / d$ ) by an amount $t o l_{1}=10^{-3}$ or more, the chain is separated into two parts: the part before and after that bond. Both parts are then rigidly shifted into the tangential direction of that bond, such that (i) the bond length after the shifting process equals 1 , and that (ii) the center of mass of the entire chain is preserved. This "repair" process for the constraint lengths is required because the method of using Lagrangian multipliers does lead to time increments which are parallel to the constraints, but due to the finite numerical time step size, higher order errors slowly add up during a simulation. Secondly, the material vectors $\vec{m}^{(3)}$ are known exactly in each time step because they equal the tangent vectors. The material vectors $\vec{m}^{(1)}$, however, are correctly initialized before the simulation, and are then iteratively parallel transported along with the dynamics of the tangent vectors. While normalizing these vectors is trivial, we cannot be certain that they do not slowly acquires a tangential component over the course of the simulation due to numerical errors. Thus, whenever any $\left|\vec{m}^{(1)} \cdot \vec{m}^{(3)}\right|>t o l_{2}=10^{-5}$, we subtract the tangential component via

$$
\vec{m}^{(1)} \mapsto \vec{m}^{(1)}-\left(\vec{m}^{(1)} \cdot \vec{m}^{(3)}\right) \vec{m}^{(3)}
$$

and normalize $\vec{m}^{(1)}$ afterwards. The remaining material vector $\vec{m}^{(2)}$ is found as the cross product of the other two.

\footnotetext{
${ }^{1}$ According to the detailed balance condition, the probability outflux from a state close to a region of zero probability must be lowered.
} 


\subsection{Monte-Carlo sampling}

In order to sample the Boltzmann distribution in presence of excluded volume effects, we make use of an algorithm which is called the Metropolis Monte-Carlo, Markov-Chain-Monte-Carlo or simply Monte-Carlo algorithm [126]. We simply refer to it as MC sampling. MC sampling constitutes an efficient method to sample a given probability distribution defined on a high-dimensional space $\Omega$. It only requires that the values of the (not necessarily normalized) probability distribution can be numerically evaluated. For the Boltzmann distribution, this condition breaks down to the energy function $E$ being evaluable.

The MC sampling algorithm is shown in figure 4.2 as a flowchart and works as follows: A discrete random walk $L:=\left\{x_{i} \mid i=1,2,3, \ldots\right\}$ within $\Omega$ is designed in such a way that the probability to go from a state $A$ to a state $B$ is the same as going from $B$ to $A$. The index $i$ is a step index, not time, and $x$ parametrizes the model chain. The random walk must satisfy the detailed balance condition:

$$
\begin{array}{rlrl}
\operatorname{flux}(A \rightarrow B) & =\operatorname{flux}(B \rightarrow A) \\
\Leftrightarrow \quad & p(A) \cdot k(A, B) & =p(B) \cdot k(B, A) \\
\Leftrightarrow & \frac{k(B, A)}{k(A, B)} & =\frac{p(A)}{p(B)} \stackrel{!}{=} \exp \left(-\frac{E(B)-E(A)}{T}\right) .
\end{array}
$$

The transition rate $k$ is then written as the product

$$
k(A, B)=k^{\text {trial }}(A, B) \cdot p^{\text {accept }}(A, B)
$$

where $k^{\text {trial }}(A, B)$ is isotropic and uniformly distributed around $A$, describing trial steps from point $A$ to a point $B$ in its vicinity. It is crucial that these trial steps are unbiased and allow all points within $\Omega$ with non-zero probability to be reached by the algorithm. In particular, this means that $k^{\text {trial }}(A, B)=k^{\text {trial }}(B, A)$, and thus the detailed balance condition above reduces to

$$
\frac{p^{\operatorname{accept}}(B, A)}{p^{\operatorname{accept}}(A, B)}=\exp \left(-\frac{E(B)-E(A)}{T}\right)
$$

After a trial step has been chosen, it is accepted with probability $p^{\text {accept }}(A, B)$, or otherwise rejected and a new trial step starting from $A$ is attempted. The core idea of MC sampling is to implement unbiased trial steps and then cleverly choose the functional form of $P^{\text {accept }}(A, B)$ such that detailed balance is obeyed while the ratio $p^{\text {accept }}(A, B) / p^{\text {accept }}(B, A)$ equals the Boltzmann factor. This condition is fulfilled for

$$
P^{\text {accept }}(B, A)=\min \left(1, \exp \left(-\frac{E(B)-E(A)}{T}\right)\right) \quad,
$$

which can be easily checked for both $E(A) \leq E(B)$ and for $E(A)>E(B)$. Therefore, it suffices that any chain conformation's energy can be computed in order to sample the Boltzmann distribution. 


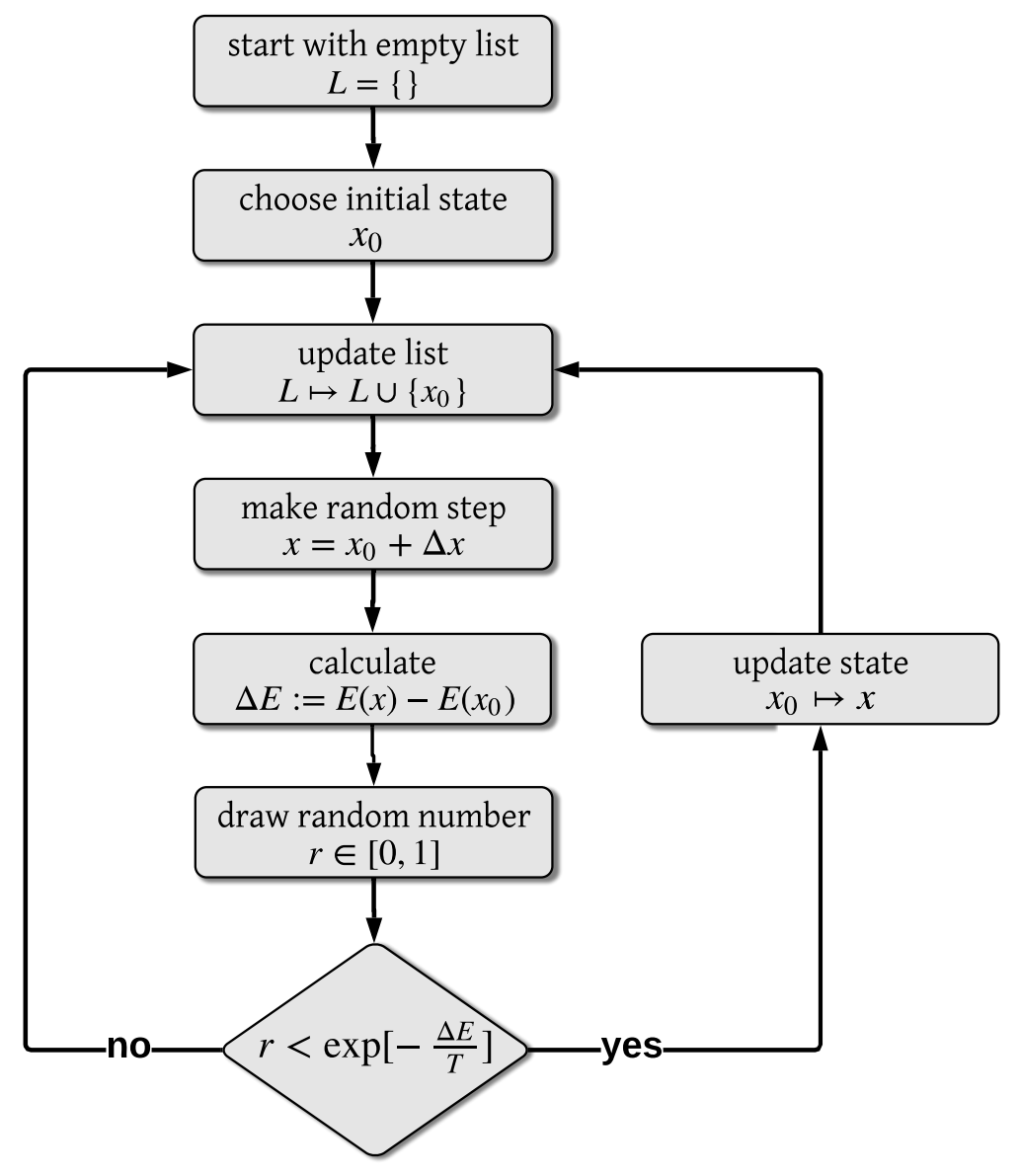

Figure 4.2: This flow chart shows the algorithm called Monte-Carlo (MC) sampling. Starting at the top, an arbitrary state of the system (e.g. a straight chain) is initiated. The state's parametrization $\vec{x}$ is then repeatedly varied in a random manner (trial steps) leading to a new state. If the new state's energy is lower than the energy of the current state, the trial step is accepted and the current state of the system is updated to this new state. If the energy is higher than before, the trial step is only accepted with probability $\exp (-\Delta E / T)$ and otherwise rejected. Before each trial step, the current state is saved to a list, regardless if the preceding trial step was successful or not. After a sufficient number of trial steps, this list is saved to an external file. Its elements are distributed according to the Boltzmann distribution. 
In this thesis, the parametrization used to represent chain conformations within $\Omega$ is the following: A chain consisting of $N$ beads is constructed via its $N-1$ tangent vectors, where the first tangent vector is held fixed because the chain's global orientation in space is irrelevant for its energy function. The remaining $N-2$ tangent vectors are represented in spherical coordinates via $N-2$ torsional (azimuthal) angles $\phi$ and $N-2 \operatorname{cosines}$ of bending (polar) angles $\cos \theta$ respectively. The polar axis for each bond is the previous bond's tangent vector, and the azimuthal angle of tangent vector $\vec{t}_{i}$ is its angle to the plane spanned by $\vec{t}_{i-1}$ and $\vec{t}_{i-2}$. Trial moves consist of changing these $2 N-4$ values by independently and simultaneously adding random numbers to each of them. These random numbers are uniformly distributed on the interval $[-\Delta, \Delta]$, and we employ reflecting boundary conditions for $\cos \theta$ in order to stay in the interval $[-1,1]$. Note that, without further discrimination, these trial moves are unbiased and lead to a uniform distribution of $\cos \theta$ and $\phi$, which is the correct equilibrium distribution on a sphere's surface (see the footnote on page 28) in the absence of bending energy and excluded volume effects. The trial moves are then accepted or rejected using the Boltzmann factor, and thus detailed balance is achieved. For calculating the Boltzmann factor, the energy of chain conformations where any two beads overlap is set to infinity such that corresponding trial moves are automatically rejected:

$$
p(\cos \vec{\theta}, \vec{\phi})= \begin{cases}0 & \text { if any two beads overlap } \\ \frac{1}{Z} \exp \left(-\frac{E}{T}\right) & \text { otherwise }\end{cases}
$$

Whenever MC sampling is used, the value of $\Delta$ is chosen anew such that the average acceptance rate of trial moves is around $30 \%$. The average acceptance rate of the MC sampling algorithm does not influence the sampled distribution, but does determine the efficiency (speed) with with different regions of $\Omega$ are sampled. It is typically larger for small step sizes $\Delta$ because in that case, the energy difference $E(B)-E(A)$ is small and the Boltzmann factor close to 1 . We remark that a Langevin simulation samples the Boltzmann ensemble as well, but is a computational overkill when used for this purpose alone. That is because MC sampling allows for much larger "time steps" and does not require the calculation of forces, diffusion matrices, Choleski decompositions and Lagrangian multipliers. Note also that the MC sampling algorithm is not altered by the presence or absence of the bound-end constraint, demonstrating that it results in correct statistics, not correct dynamics! To give the reader an intuition, in practice (using my code) a sufficiently long Langevin simulation takes a few days, and sufficiently long MC sampling a few minutes. 


\subsection{Hydrodynamic radius of a polymer chain}

As we found in section 2.2.7 on page 52, the Stokes-Einstein relation relates any object's hydrodynamic radius $R_{H}=T /(6 \pi \eta D)$ to its diffusion coefficient $D$, which in turn is defined via the object's mean-square displacement over time ( $6 D=\left.\lim _{t \rightarrow \infty} \partial_{t^{\prime}}\left\langle\vec{R}^{2}\right\rangle\right|_{t}$ ) and is related to the $3 \times 3$-dimensional diffusion matrix $\mathbf{D}$ via $D=\operatorname{Tr} \mathbf{D} / 3$. Before evaluating these expressions for a polymer chain, let us present a result from De la Torre and coworkers [127, 128]. They have shown that the scalar diffusion coefficient $D$ of any rigid conformation of a polymer can be directly calculated from its $3 N \times 3 N$-dimensional diffusion matrix $\mathbf{D}$ as follows. Given the collection of forces on all beads, $\vec{F}=\left(\vec{F}_{1}, \ldots, \vec{F}_{N}\right)$, the collection of velocities $\vec{v}$ is given by $\vec{v}=\beta \mathbf{D} \cdot \vec{F}$. Inversion delivers $\vec{F}=\boldsymbol{\Gamma} \cdot \vec{v}$ where $\boldsymbol{\Gamma}=T \mathbf{D}^{-1}$ is the $3 N \times 3 N$-dimensional generalized friction tensor, consisting of $N^{2} 3 \times 3$-blocks $\boldsymbol{\Gamma}_{i j}$. A rigid translation with velocity $\vec{v}_{R}$ (all beads move with same velocity) is thus related to the total force $\vec{F}_{R}=\sum_{i} \vec{F}_{i}$ acting on the entire molecule via

$$
\vec{F}_{R}=\sum_{i, j=1}^{N} \boldsymbol{\Gamma}_{i j} \vec{v}_{R}=: \boldsymbol{\Gamma}_{R} \vec{v}_{R}
$$

Hence, the connection between total force and rigid translational velocity is described via the $3 \times 3$-dimensional friction tensor $\boldsymbol{\Gamma}_{R}=\sum_{i, j=1}^{N} \boldsymbol{\Gamma}_{i j}$. Therefore, the diffusion coefficient of one chain conformation is obtained as

$$
D=\frac{1}{3} \operatorname{Tr} \mathbf{D}_{R}
$$

where $\mathbf{D}_{R}=T \boldsymbol{\Gamma}_{R}^{-1}$ is the rigid molecule's $3 \times 3$-dimensional diffusion tensor. Our polymer chains are not rigid, however, but undergo conformational dynamics, making $\mathbf{D}_{R}$ time-dependent because the diffusion matrix $\mathbf{D}$ is a function of the Cartesian coordinates of the chain's vertices. We will now make an argument that in this case, the (long-term) diffusion coefficient is given by the average over the Boltzmann ensemble:

$$
D=\langle D(\vec{r})\rangle=\frac{1}{3}\left\langle\operatorname{Tr} \mathbf{D}_{R}(\vec{r})\right\rangle
$$

The diffusive chain dynamics of the polymer is a series of infinitesimal increments at different times with different chain conformations such that

$$
\begin{aligned}
\vec{R}(t) & =\int_{0}^{t} \mathrm{~d} \vec{R} \\
\text { and } \quad\left\langle\vec{R}^{2}\right\rangle & =\left\langle\int_{0}^{t} \int_{0}^{t} \mathrm{~d} \vec{R}\left(t^{\prime}\right) \cdot \mathrm{d} \vec{R}\left(t^{\prime \prime}\right)\right\rangle
\end{aligned}
$$

Here, $\vec{R}$ is any coordinate of the chain, for example one of its vertices. For short times, the individual vertices undergo conformational dynamics, which are faster than the chain's translational diffusion we seek to study here. The reason for this is that the polymer is not a rigid object, and the friction associated with the movement of a single bead is smaller than that associated 
with movements of the chain as a whole. This circumstance can be observed in the simulated curve $\left\langle\vec{R}^{2}\right\rangle(t)$ displayed in figure 4.7 on page 104, and in the additional figures in appendix $\mathrm{F}$; the function $\left\langle\vec{R}^{2}\right\rangle(t)$ is concave with its slope decreasingly converging towards $6 D$. It would therefore be incorrect to insert the Langevin equation for $\mathrm{d} \vec{R}$ as it describes the vertices' short term dynamics - doing so would deliver an average of initial slope $\partial_{t}\left\langle\vec{R}^{2}\right\rangle(0)$ for different chain conformations. To obtain instead a chain conformation's long-term slope $\partial_{t}\left\langle\vec{R}^{2}\right\rangle(\infty)$, we make use of De La Torre's result above ${ }^{2}$ and obtain

$$
\begin{aligned}
\left\langle\vec{R}^{2}\right\rangle & =\left\langle\int_{0}^{t} \int_{0}^{t} \mathrm{~d} \vec{R}\left(t^{\prime}\right) \cdot \mathrm{d} \vec{R}\left(t^{\prime \prime}\right)\right\rangle \\
& =\int_{0}^{t}\left\langle\mathrm{~d} \vec{R}^{2}\right\rangle\left(t^{\prime}\right) \\
& =6 \int_{0}^{t} D\left(t^{\prime}\right) \mathrm{d} t^{\prime} \\
& =6\langle D\rangle t \\
& =6 \cdot \frac{1}{3}\left\langle\operatorname{Tr} \mathbf{D}_{R}\right\rangle t
\end{aligned}
$$

Here, the statistical independence of increments at different times was used, which is justified for translational dynamics of a rigid chain, but not necessarily for intrachain dynamics. Finally, we find the hydrodynamic radius of a polymer chain to be:

$$
\begin{aligned}
R_{H} & =\frac{T}{6 \pi \eta D} \\
& =\frac{T}{2 \pi \eta\left\langle\operatorname{Tr} \mathbf{D}_{R}\right\rangle} \\
& =\frac{3 T}{6 \pi \eta D_{0}\left\langle\operatorname{Tr} \mathbf{D}_{R}^{\prime}\right\rangle} \\
& =a \frac{3}{\left\langle\operatorname{Tr} \mathbf{D}_{R}^{\prime}\right\rangle} \\
\text { where } \quad \mathbf{D}_{R} & =T \Gamma_{R}^{-1}, \\
\boldsymbol{\Gamma}_{R} & =\sum_{i, j=1}^{N} \boldsymbol{\Gamma}_{i j} \\
\text { and } \quad \boldsymbol{\Gamma} & =T \boldsymbol{D}^{-1},
\end{aligned}
$$

and $D_{0}=T /(6 \pi \eta a)$ as before. In numerical practice, the average $3 \cdot\left\langle\operatorname{Tr} \mathbf{D}_{R}^{\prime}\right\rangle^{-1}$ is evaluated using $\mathrm{MC}$ sampling. In order to be converted into physical units, the so-found value only needs to be multiplied with the single bead hydrodynamic radius $a$, which also enters the diffusion matrix during the averaging process.

\footnotetext{
${ }^{2}$ In De La Torre's picture of a rigid chain, the mean square displacement is a linear function of time with a constant slope for all times. Thus its short-term and long-term behaviors are identical.
} 
We remark that in the absence of hydrodynamic interactions, $\mathbf{D}$ and $\boldsymbol{\Gamma}$ are diagonal such that $\Gamma_{R} \propto N$ and thus $R_{H} \propto N$. To be more precise, one obtains $R_{H}=\sum_{i} a_{i}$ where $a_{i}$ is the i-th bead's hydrodynamic radius. For intuition, a number of polymer chain conformations together with their respective hydrodynamic radii are displayed in figure 4.3.
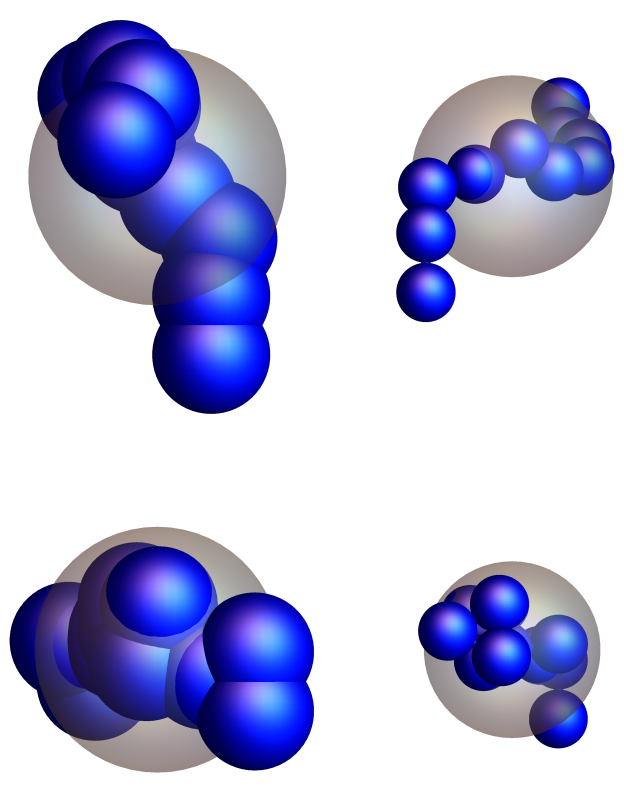
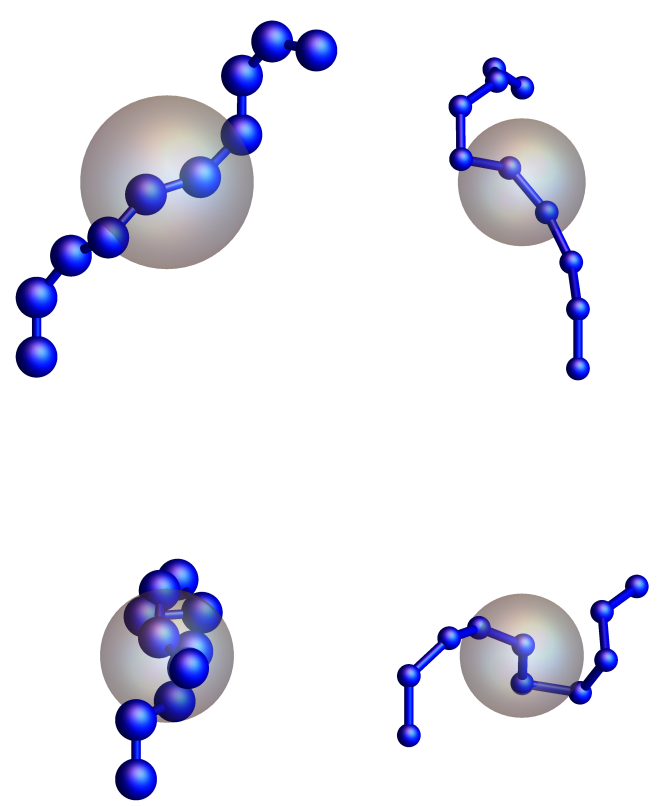

Figure 4.3: 8 polymer chains, shown in blue, consisting of 10 beads each. The rods connecting the beads are only shown for visual purposes. The hydrodynamic bead radius $a$ decreases from left to right (displayed bead radii are $a \in\{1,0.5, \sqrt{2} / 4,0.2\}$ bond lengths), which decreases the hydrodynamic radius of the chain (shown in gray, calculated using equation 4.23 for a single conformation). More compact conformations can be seen on the bottom and more elongated ones on top. On average, the gray sphere would diffuse as fast as the respective polymer chain in that conformation.

The polymer's diffusion coefficient can also be obtained directly from a Langevin simulation by evaluating $6 D=\left.\lim _{t \rightarrow \infty} \partial_{t^{\prime}}\left\langle\vec{R}^{2}\right\rangle\right|_{t}$. A simulated trajectory used for this purpose needs to be long enough to sample a sufficiently large set of chain conformations and noise realizations, however, which is numerically much more expensive than using MC sampling.

A quantitative analysis of the dependence of $R_{H}$ on the chain's parameters $a, \sigma$ and $N$ can be found in chapter 5.1 on page 107. 


\subsection{Contact rates}

Next we demonstrate how the contact rate $k_{+}$is calculated from a simulated trajectory $\vec{r}(t)$. For better understanding, one such trajectory's end-to-end distance is shown in figure 4.4.

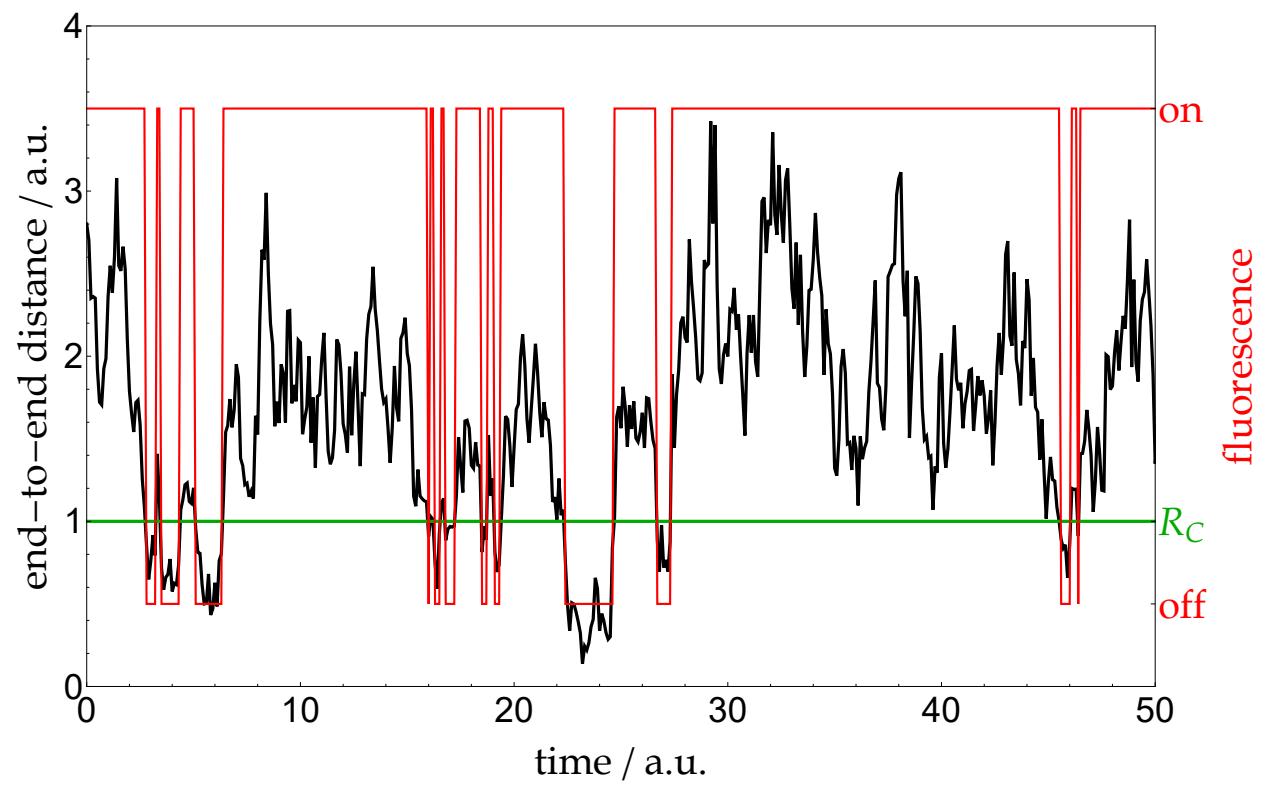

Figure 4.4: A simulated trajectory of the end-to-end distance (black) in a chain with parameters $\sigma=0, a=1$ and $N=5$. Shown in red is the corresponding fluorescence intensity (state) of the chain, using a critical threshold (green line) of $R_{\mathrm{C}}=1$.

As we have seen in section 2.5.3 (page 74), the experimentally measured rate $k_{+}$with which a fluorophore and quencher meet is given by

$$
k_{+}=\frac{1}{\langle\tau\rangle}
$$

When starting in an open state, $\tau$ denotes the time until the first fluorophore-quencher encounter, meaning that their distance deceeds the critical contact radius $R_{C}$. The average runs over the Boltzmann ensemble of all open initial states. Note that the rate $k_{+}$is therefore not the frequency with which the end-to-end distance crosses the threshold $R_{C}$, as this rate would be very high, considering that in general every opening event is immediately followed by several re-closing events! Instead, the rate $k_{+}$must be understood like this: Given an ensemble of chains which are somewhere in the open state, $k_{+}$is the rate with which this ensemble loses trajectories which have never left the open state. For a single trajectory, let us denote the times when the state of the chain (open or closed) is changing in either direction as $t_{k}$. A simulation thus delivers a list of "threshold crossing events" $\left\{t_{1}, t_{2}, t_{3}, \ldots\right\}$. 
Next, we divide the time axis into intervals $I_{k}:=\left[t_{k-1}, t_{k}[\right.$ such that the time intervals including all open and closed states respectively are

$$
\begin{aligned}
I^{\text {open }} & =\bigcup_{k \text { odd }} I_{k} \\
\text { and } \quad I^{\text {closed }} & =\bigcup_{k \text { even }} I_{k}
\end{aligned}
$$

Here, we assumed that the simulation starts in the open state which in practice is almost always the case. If, against all odds, a simulation starts in the closed state, we simply start the list of events with $t_{1}=0$ and proceed as usual. For any open initial state at time $t \in I^{\text {open }}$, the time until the next closing event is $\tau(t)=\min _{t_{k}>t} t_{k}-t$ and we note that within any open interval $I_{k}$, the average time until the next event is given by

$$
\langle\tau\rangle_{k}=\frac{\left|I_{k}\right|}{2}=\frac{t_{k}-t_{k-1}}{2}
$$

Averaging $\tau$ over the set of all open initial conditions $I^{\text {open }}$ then yields

$$
\begin{aligned}
\langle\tau\rangle & =\frac{1}{\left|I^{\text {open }}\right|} \int_{I^{\text {open }}} \tau\left(t^{\prime}\right) \mathrm{d} t^{\prime} \\
& =\frac{\sum_{k \text { odd }} \int_{I_{k}} \tau\left(t^{\prime}\right) \mathrm{d} t^{\prime}}{\sum_{k \text { odd }} t_{k}-t_{k-1}} .
\end{aligned}
$$

The integrals in the enumerator can easily be evaluated as

$$
\int_{I_{k}} \tau\left(t^{\prime}\right) \mathrm{d} t^{\prime}=\frac{\left|I_{k}\right|}{\left|I_{k}\right|} \int_{I_{k}} \tau\left(t^{\prime}\right) \mathrm{d} t^{\prime}=\left(t_{k}-t_{k-1}\right) \cdot\langle\tau\rangle_{k}=\frac{\left(t_{k}-t_{k-1}\right)^{2}}{2} .
$$

Hence, the contact rate $k_{+}$is given by the simple expression

$$
k_{+}=\langle\tau\rangle^{-1}=2 \frac{\sum_{k \text { odd }} t_{k}-t_{k-1}}{\sum_{k \text { odd }}\left(t_{k}-t_{k-1}\right)^{2}} \quad .
$$

When running a simulation, one thus only needs to save the event times $t_{k}$ (closing and opening events!) into a text file in order to determine the contact rate $k_{+}$. It should be noted that the trajectory length should be long compared to $\langle\tau\rangle$ in order not to incorrectly underrepresent long first passage times $\tau$. The contact rate's error bars are obtained as follows: We calculate $M$ independent values for $k_{+}$, estimate their standard deviation, and divide it by $\sqrt{M}$. The $M$ independent values stem from independent trajectories with identical parameters, or from dividing a single sufficiently long trajectory into $M$ equally long parts.

Since the numerical values of $t_{k}$ are nondimensional, the contact rate $k_{+}$above must be multiplied with $t_{0}^{-1}$ in order to be compared with experimental results.

Clearly, it can be expected that $k_{+}$depends on the chosen threshold $R_{C}$. This dependence is investigated in section 5.2 (see page 127). In the experimentally relevant regime, we will find the simple scaling law $k_{+} \propto R_{C}^{2}$ to be a good approximation. 


\subsection{Benchmarks}

Our simulation algorithm generates trajectories which obey the constrained Langevin equation. Due to the presence of geometric constraints and the nonlinearity of both the diffusion matrix and the force terms, a full analytical solution is not available. Nonetheless, some properties of the solution are known to us, and it is possible for us to validate our algorithm by comparing numerical with theoretical results.

Firstly, simulated chains have to fulfil the imposed geometric constraints. Secondly, the simulated trajectories' bond angles have to sample their Boltzmann distribution. Thirdly, the slope of the chain's long-term mean squared displacement has to be a linear function of time and yield the correct scalar diffusion coefficient. Fourthly, although no analytical values for the contact rates are available, our numerical values have to converge as the numerical time step size $\Delta t$ is decreased. Unless stated otherwise, we consider chains with uniform bond lengths and hydrodynamic bead radii, and without excluded volume effects. The free parameters are therefore $a, \sigma$ and $N$.

Constraints With the help of Lagrangian multipliers, our numerical algorithm projects the increments $\mathrm{d} \vec{r}$ such that they have no component perpendicular to the geometric constraints. Due to the geometric nonlinearity of these constraints and the finite time step size utilized, higher-order errors exist and accumulate during a simulation. Our algorithm repairs the slightly violated constraints whenever a tolerance of $t o l_{1}=10^{-3}$ is exceeded. The criterion we test here is thus that the deviation $\delta g$ from a constraint, as a function of time, should take on values between 0 and $t_{0 l}$.

\begin{tabular}{cc}
\hline$\sigma$ & $\langle|\delta g|\rangle /$ tol \\
\hline 0 & 0.374 \\
0.25 & 0.366 \\
0.5 & 0.357 \\
0.75 & 0.347 \\
1 & 0.344 \\
\hline
\end{tabular}

Table 4.1: The time-averaged deviations from the inextensility constraints for different chain stiffnesses $\sigma$.

The time-averaged values of $\delta g$, where $g$ denotes the inextensibility constraints in freely swimming chains, were monitored and are shown in table 4.1. The chains consist of $N=10$ beads with hydrodynamic radii $a=1$, and the bending stiffness $\sigma$ was altered between 0 and 1 . The displayed values were monitored after a time step was performed and before the constraints were potentially repaired. We also verified that all increments $\mathrm{d} \vec{r}$ indeed have no component perpendicular to the constraints after adding the Lagrangian multipliers to the forces. 
Boltzmann distribution As before (see the section on MC sampling, page 92), we represent each tangent vector in spherical coordinates $(\phi, \theta)$, and the corresponding Boltzmann distribution for each bond is given by

$$
p(y=\cos \theta, \phi)=\frac{1}{Z} \exp \left(-\frac{\sigma}{2 d}\left(2 \tan \left(\frac{\arccos y}{2}\right)\right)^{2}\right) \quad .
$$

It notably does not depend on the azimuthal angles $\phi$ whose distribution must hence be uniform. Chains with $N=10$ and $a=1$ were simulated for different values of $\sigma$. The simulated histograms of values for $\cos \theta$ and $\phi$ were taken over all times and all vertices, and are shown in figure 4.5. We show the bending angles' cosines instead of the bending angles themselves because without a bending energy $(\sigma=0)$, the cosines would be uniformly distributed, and thus the asymmetry introduced by the bending energy $(\sigma>0)$ becomes more clear.

The histograms for each individual bond have the same form, but are less well converged since there is less data available from the same trajectory. Choosing a different hydrodynamic bead radius for the first bead (i.e. attaching a fluorophore) does not alter the histograms (data not shown).

To verify that the excluded volume effect is correctly implemented, we additionally simulated a trimer $(N=3)$ without bending stiffness $(\sigma=0)$ and with an excluded volume radius for each bead of $R_{\mathrm{EV}}=1 / 2$. Thus, purely geometrically, bending angles exceeding that of an equilateral triangle $(\theta=2 \pi / 3 \Rightarrow \cos \theta=-0.5)$ are forbidden, and all larger cosines of bending angles are equally probable. The simulated histogram is shown in figure 4.6.

Diffusion coefficients Here, we simulated chains which resemble those modeling GS-repeats in section 5.2, using short chain lengths for good convergence. They have parameters $N=5$ and $N=16, \sigma=1$, an excluded volume radius $R_{\mathrm{EV}}=\sqrt{2} / 4$ and the following hydrodynamic properties: (i) no hydrodynamic interactions, $a=0$; (ii) hydrodynamic interactions, $a=1$; and (iii) hydrodynamic interactions and a fluorophore attached, $a=1, a_{1}=6 / 3.8, d_{1}=7 / 3.8$. In each simulation, the mean square displacements (MSD) $\left\langle\vec{R}^{2}\right\rangle$ of the chain's center of mass and of the first bead were calculated and they correctly turned out to be linear functions of time in the long-time limit. For each parameter set, this function was compared with the slope predicted by equation 4.22 (page 96). This is achieved by dividing the MSD by $6 D$ such that its slope must converge towards 1 . An offset between the MSD and a diagonal line is allowed - the MSD's initially higher slope reflects the fast intrachain dynamics. Figure 4.7 shows a successful test of this criterion for $N=5$ (simulation results for short chains converge faster) and $a=1$. The (similarly looking) tests for the other parameter sets are shown in appendix F. 


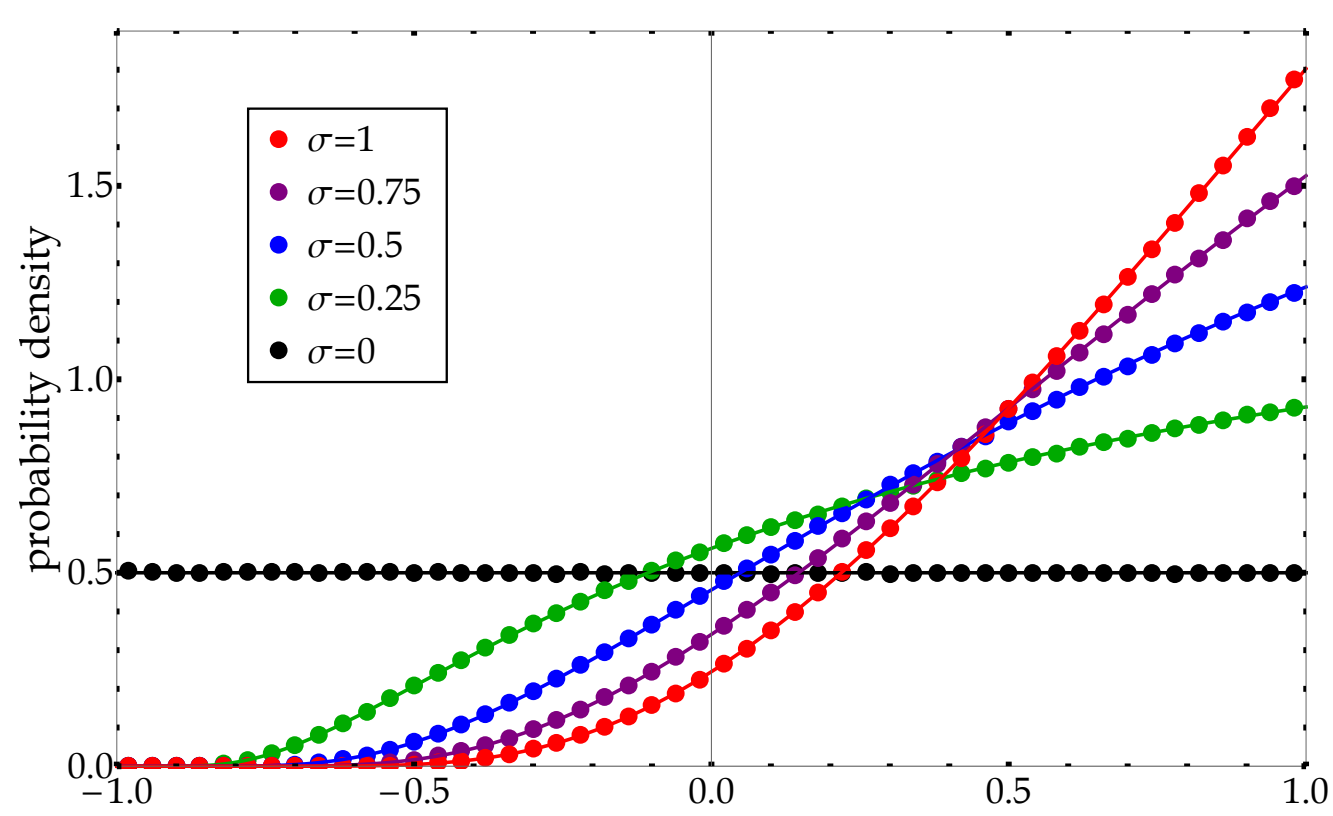

cos( bending angle )

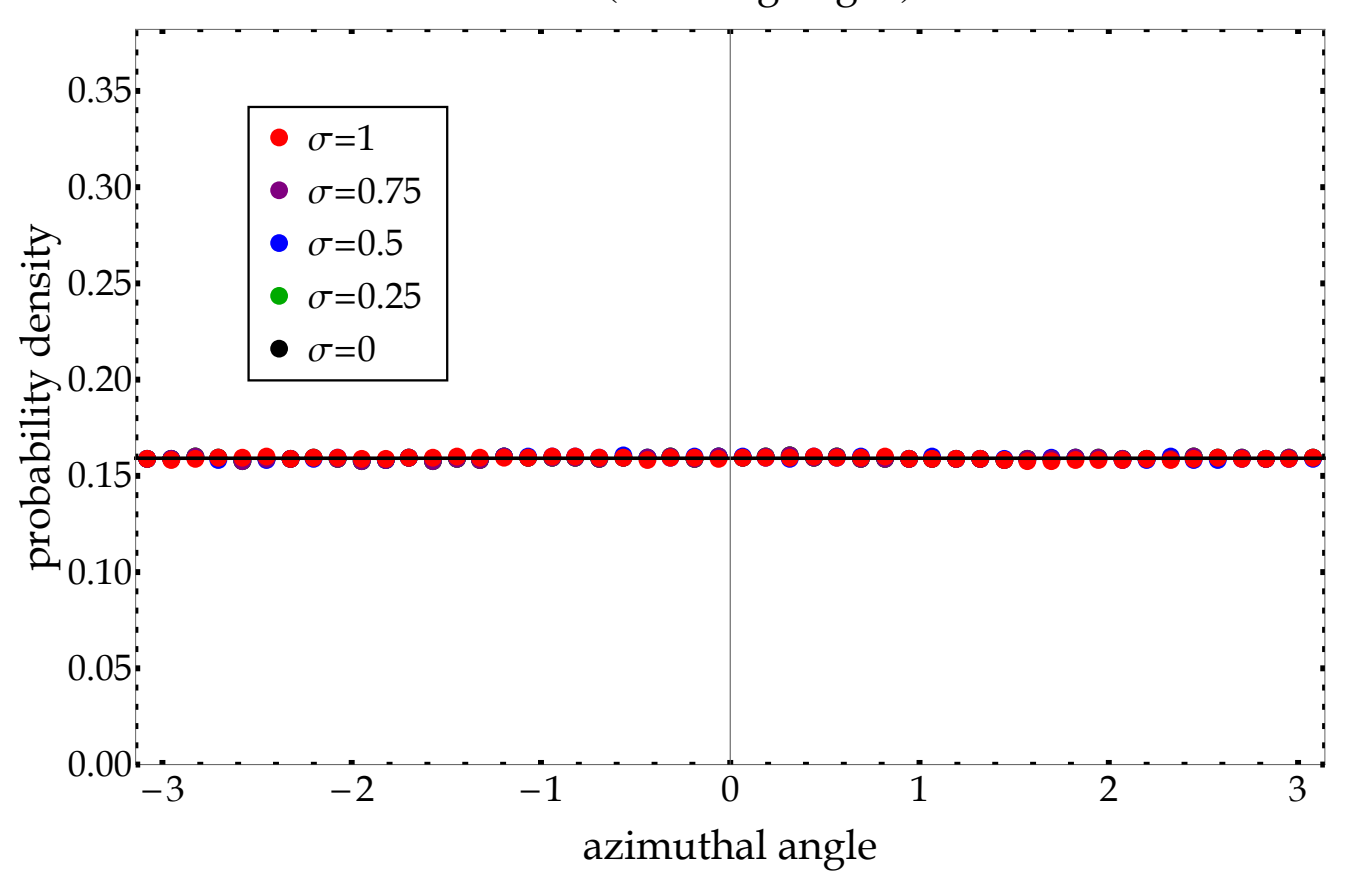

Figure 4.5: Validation that the simulated trajectories sample the Boltzmann distribution. Top: Histograms (dots) of simulated cosines of bending angles $\theta$ versus their Boltzmann distribution $\propto \exp \left(-2 \sigma \tan (\theta / 2)^{2}\right)$ (lines). These simulations were performed for $N=10$ and $a=1$, thus including hydrodynamic interactions. Bottom: Histograms of simulated azimuthal angles (dots) versus their Boltzmann distribution (line), which is a constant since no energy is associated with changes of azimuthal angles. In both figures, the simulated histograms lie on the theoretically predicted curves. 


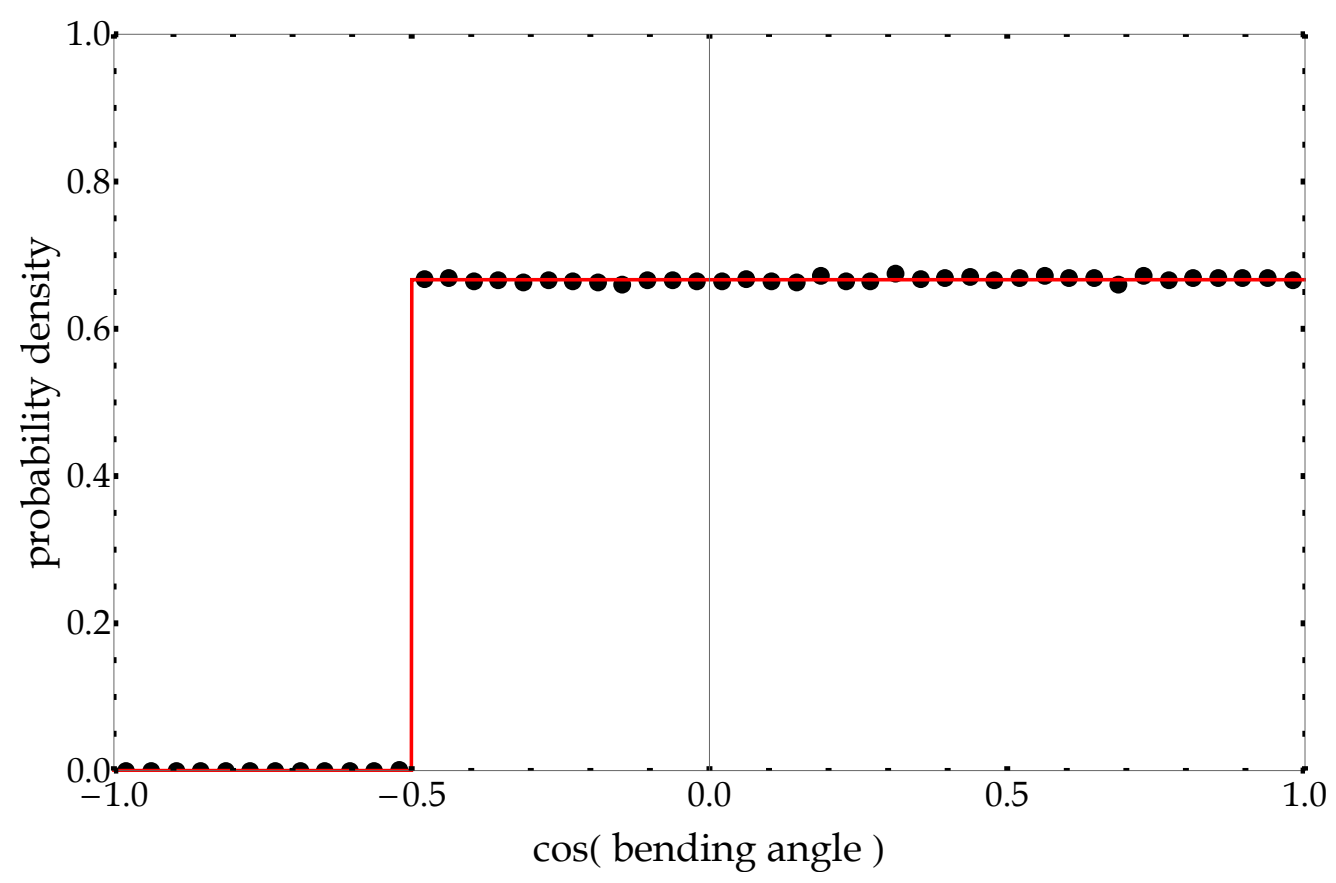

Figure 4.6: Validation that excluded volume effects are implemented correctly and the Boltzmann distribution is sampled. Here, a trimer $(N=3)$ with hydrodynamic interactions $(a=1)$ but without bending stiffness $(\sigma=0)$ was simulated. Each of the three beads has an excluded volume radius of $R_{\mathrm{EV}} / d=0.5$ such that bending angles $\theta$ with $\cos \theta<-0.5$ are not allowed. The Boltzmann distribution (red line) is thus a step function. The simulated histogram (black dots) lies on this step function. 

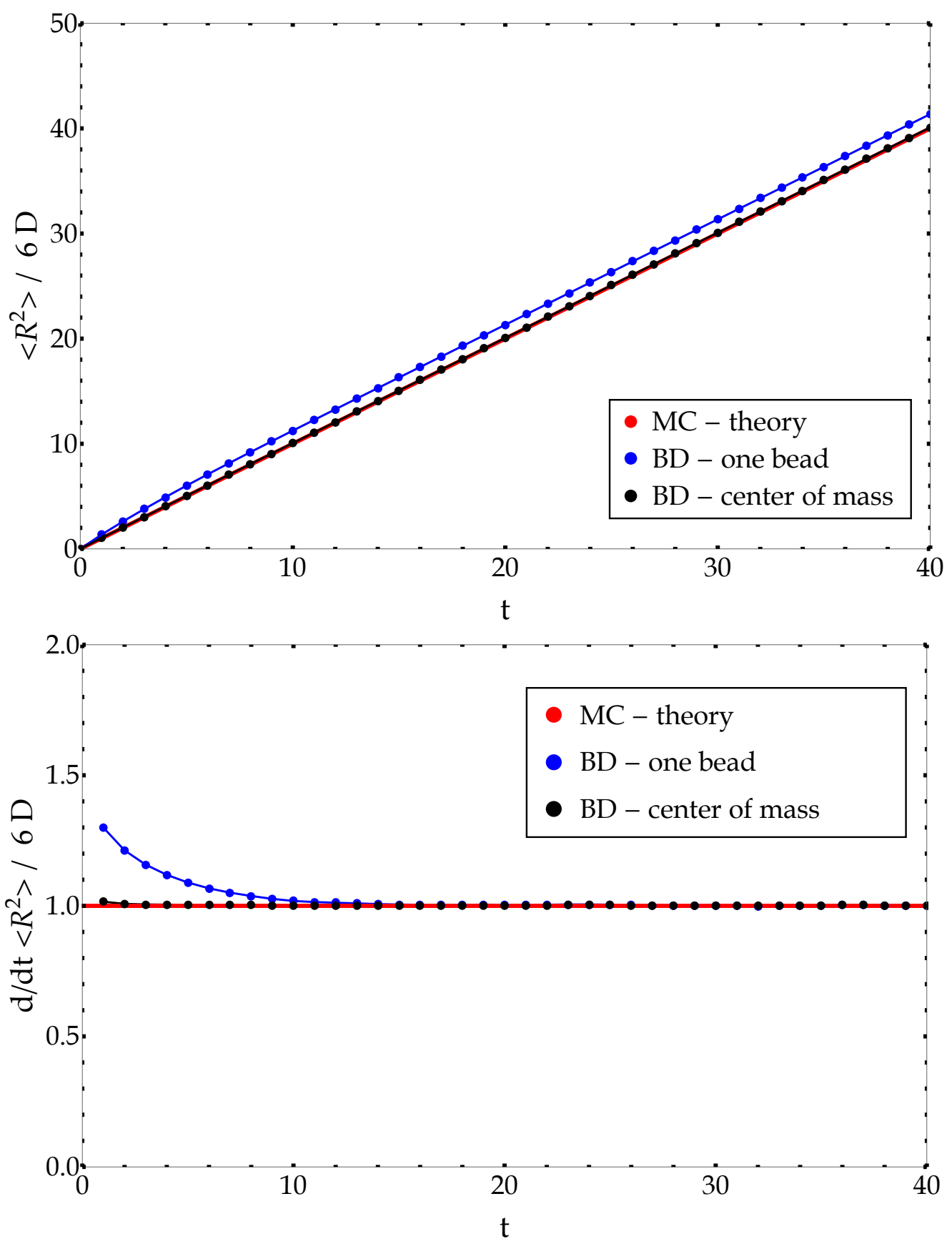

Figure 4.7: Top: The simulated ("BD" means Brownian dynamics) mean square displacement (MSD) of a chain consisting of $N=5$ beads and having parameters $a=\sigma=1$ and $R_{\mathrm{EV}}=\sqrt{2} / 4$. The blue and black dots show the MSD of the chain's first bead, and of its center of mass taken from the same trajectory. Both curves were divided by $6 D$, where $D$ is the chain's diffusion coefficient as calculated from equation 4.20 (page 95). Therefore, both curves' slopes must converge towards 1 . Bottom: The slopes of the curves from the top figure. Indeed, both the single-bead's as well as the center-of-mass' MSDs' slopes converge towards 1 . The singlebead dynamics are faster (higher slope) at first, which reflects the intrachain dynamics on short time scales. 
Time step size The end-to-end contact rates $k_{+}$for chains with identical parameters $N=10$, $a=1$ and $\sigma=1$ were simulated, thus including the nonlinearities that both hydrodynamic interactions and elastic forces impose on the numerics. The critical contact radius was set to $R_{C}=5.4 / 3.8$ because this is the same value which is later used for fitting PET-FCS data. We have systematically changed the numerical time step size $\Delta t$ in logarithmic steps from $10^{-3.5}$ to $10^{-1}$. All trajectory lengths are identical $\left(10^{5} \cdot t_{0}\right)$, but naturally, the simulated real times may differ by a factor of around three thousand. Thus, a trade-off has to be made between accuracy and numerical expense. Contact rate values for larger time steps are smaller because threshold-crossing events are more likely to go unnoticed the coarser the temporal resolution is. Most importantly, figure 4.8 shows that the value of the simulated contact rate indeed converges as $\Delta t$ decreases, and that the value $\Delta t=10^{-3} t_{0}$ is sufficiently accurate. This value was also used to validate the sampled Boltzmann distribution, correct diffusion coefficient, fulfilled constraints and excluded volume effects in the previous paragraphs, and hence we choose $\Delta t=10^{-3} t_{0}$ throughout this thesis.

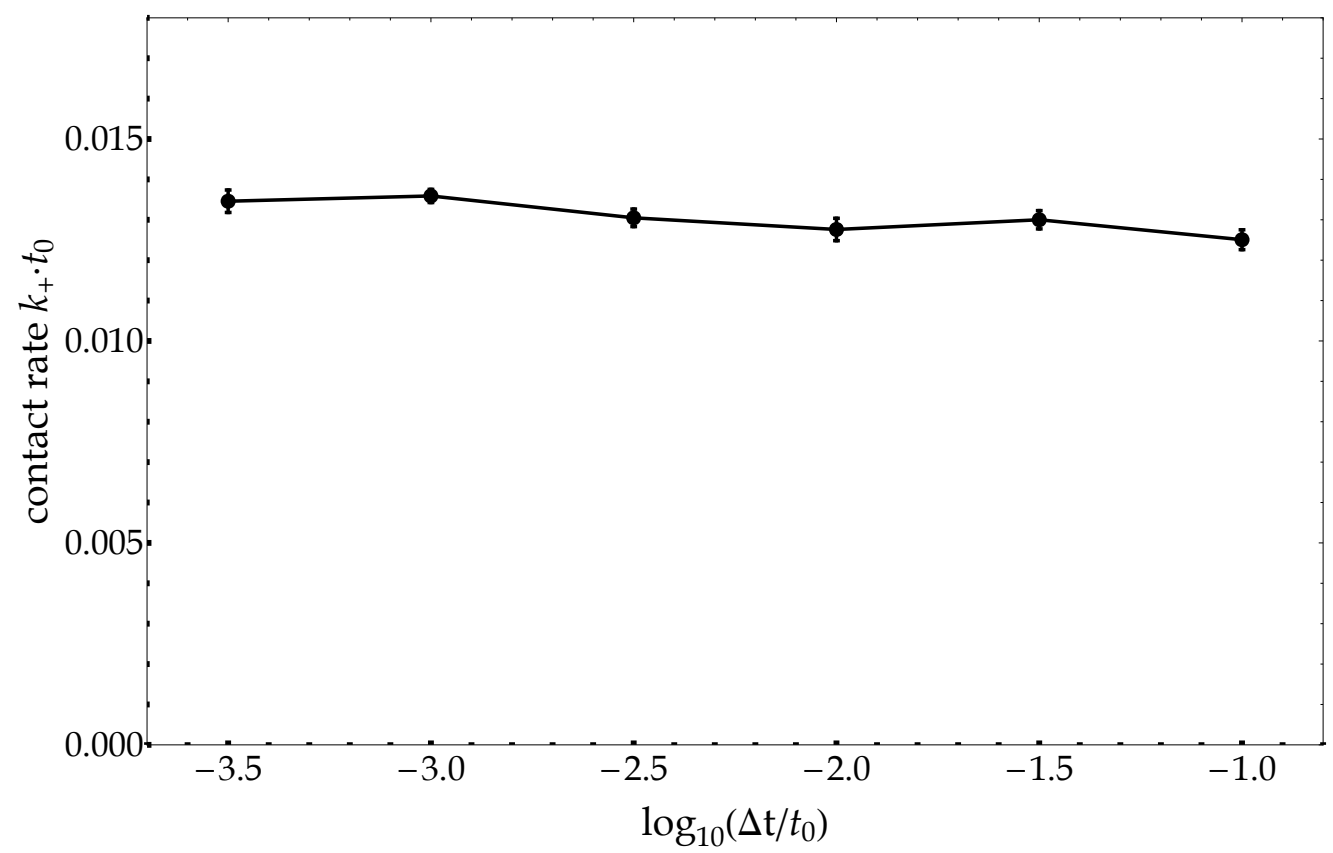

Figure 4.8: The simulated contact rate for $N=10, R_{\mathrm{EV}}=0$ and $a=\sigma=1$ as a function of the numerical time step $\Delta t$. Both the time step and contact rate are given in dimensionless units ( $t_{0}$ and $t_{0}^{-1}$ respectively). All six trajectory lengths equal $10^{5} t_{0}$. Therefore the data point on the right ( $<$ two minutes) was calculated more than 3000 times faster than the one on the left (three days). 



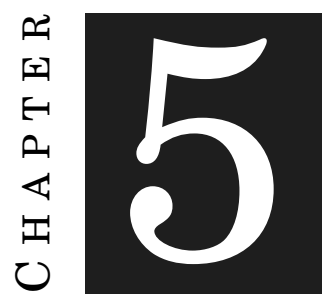

\section{Applications}

In this chapter, the results of my research related to the main theme of this thesis are presented. Side projects can be found in chapter 8 . I have not performed any experiments myself - PETFCS experiments were performed by Man Zhou, 2fFCS experiments by Arindam Ghosh, and dynaMIET experiments by Roman Tsukanov, Arindam Ghosh and Jan Christoph Thiele.

\subsection{Model behavior}

In this section, we develop a physical intuition about the model's behavior before we start fitting experimental data with it. To this end, we study the influence of the two model parameters $\sigma$ and $a$ on the model chain's hydrodynamic radius $R_{H}$ and contact rate $k_{+}$, as well as the change of $k_{+}$as the quencher position is shifted along the chain's contour. Throughout this section, we are using dimensionless units and thus measure $a$ and $\sigma$ in units of $d=1$. Excluded volume effects are not considered, and the chains consist of $N$ identical beads with equidistant bond lengths.

\subsubsection{Hydrodynamic radius}

We study the hydrodynamic radius for different values of $\sigma, a$ and $N$. Each parameter is varied while the other two are fixed. $\sigma=1$ corresponds to a persistence length of $l_{P} \approx 2$. 
Hydrodynamic radius as a function of chain length Figure 5.1 shows the functional form of $R_{H}(N)$ for $a \in\{0.5,1\}$ and $\sigma \in\{0,1\}$. For $N=1$, one naturally has $a=R_{H}$. All curves are monotonically growing, and the ones with larger bending stiffness grow faster. The reason for this is clear: An additional bead in a stiff chain is on average spatially further away from the other beads and thus the volume which is effectively occupied by the chain grows faster than for a flexible chain. Furthermore, since the volume occupied by infinitely growing chains also grows indefinitely, the functions $R_{H}(N)$ do not converge, but are unbound.

Interestingly, the difference between curves with identical $\sigma$ values but different values for $a$ seems to be reasonably constant as the chains grow in length. This is consistent with the findings in the next paragraph.

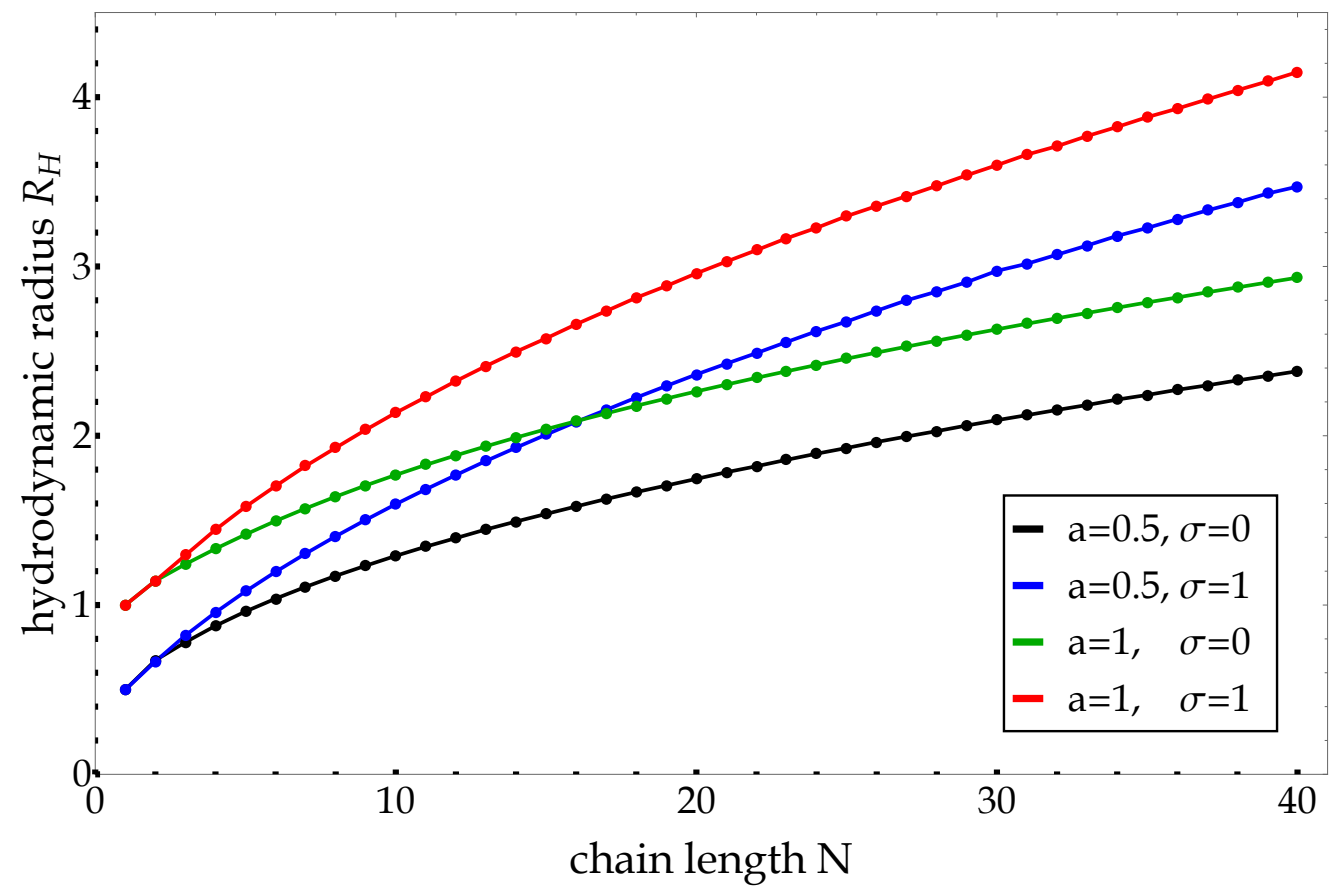

Figure 5.1: The hydrodynamic radius $R_{H}$ of model chains as a function of the number of identical beads $N$ which the chain consists of. The curves for $a=0.5$ are shown in black $(\sigma=0)$ and blue $(\sigma=1)$. The curves for $a=1$ are shown in green $(\sigma=0)$ and red $(\sigma=1)$. 
Hydrodynamic radius as a function of bead size Figure 5.2 shows the functional form of $R_{H}(a)$ for $\sigma \in\{0,1\}$ and $N \in\{10,20\}$. For $a \rightarrow 0$ one naturally has $R_{H} \rightarrow 0$. In this limit, hydrodynamic interactions are negligible which means that $R_{H}=a \cdot N$ (see page 96). We can confirm this by checking that the initial slopes of all curves $R_{H}(a)$ equal $N$. In the limit $a \rightarrow \infty$, the beads overlap entirely which makes the chain essentially behave like a single large bead with radius $a$. In this case, all individual $3 \times 3$ Rotne-Prager diffusion matrices (equation 3.4, page 83) have converged towards the unity matrix, and it is straightforward to check that $R_{H} / a \rightarrow 1$ in this limit, which our results confirm. It follows that the $R_{H}(a)$ curves do not converge but grow indefinitely. In between the two limits, the slopes of all curves decrease from $N$ to 1 . This decrease is faster for short chains because all beads overlap more quickly for growing $a$ when the chain is more compact. For the same reason, the slope's decrease is slower for stiffer chains. Already, the logical consequence is that the $R_{H}$ values themselves are larger for larger $\sigma$ values, as the next paragraph confirms. We further remark that the fact that the curves' slopes decrease (compared to the initial situation without hydrodynamic interactions) implies that the chain as a whole diffuses faster through space due to the beads' coupled, more cooperative, movement.

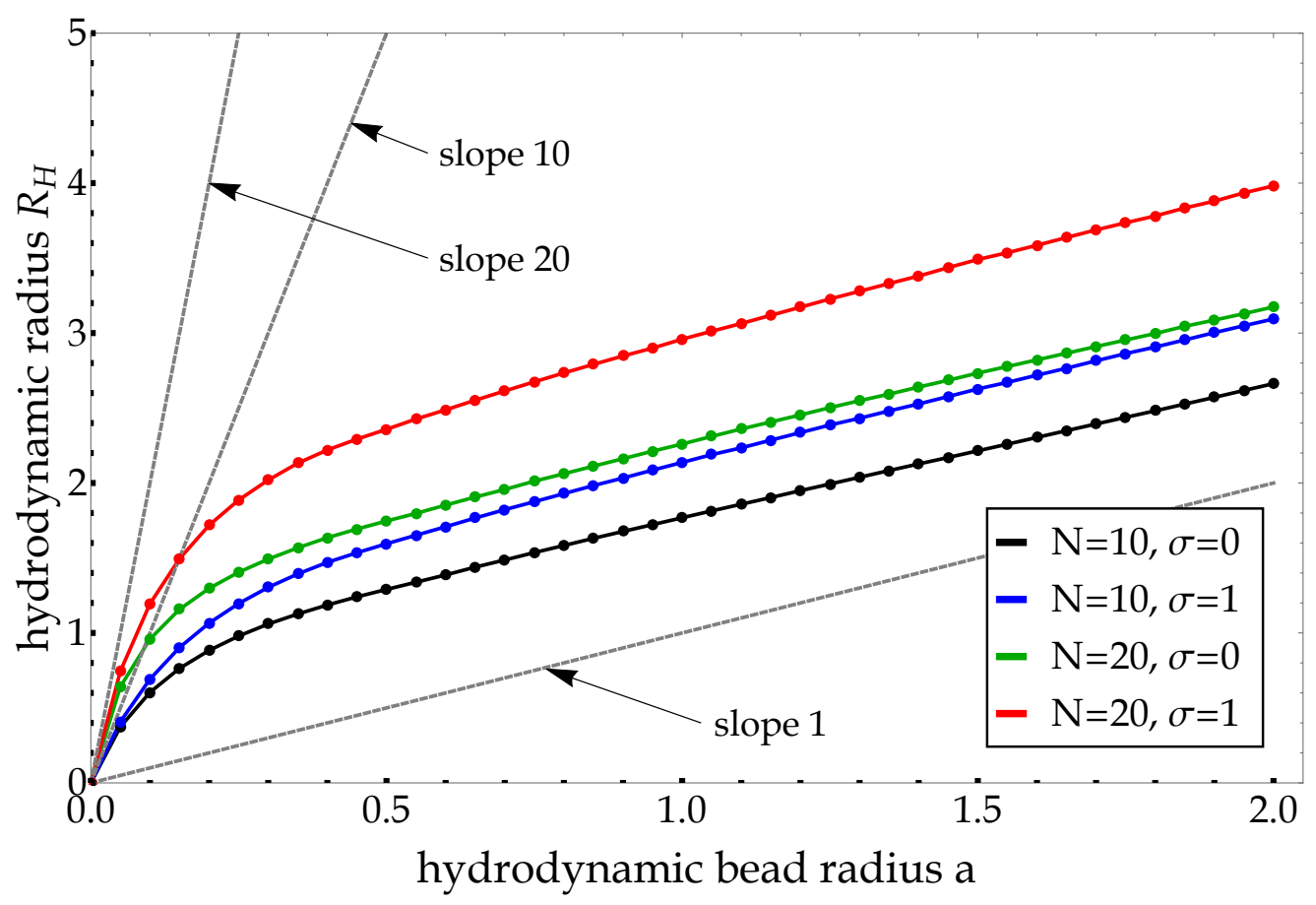

Figure 5.2: The hydrodynamic radius $R_{H}$ of model chains as a function of the hydrodynamic radius $(a)$ of each bead which the chain consists of. The curves for $N=10$ are shown in black $(\sigma=0)$ and blue $(\sigma=1)$. The curves for $N=20$ are shown in green $(\sigma=0)$ and $\operatorname{red}(\sigma=1)$. Straight lines of given reference slopes are shown for visual purposes. 
Hydrodynamic radius as a function of bending stiffness Figure 5.3 shows the functional form of $R_{H}(\sigma)$ for $a \in\{0.5,1\}$ and $N \in\{10,20\}$. For $\sigma=0$ we have at hand a purely random walk in space (FJC). For growing bending stiffness the chains swell and take up more effective volume in space - the hydrodynamic radius grows. In the limit $\sigma \rightarrow \infty$, the chains' conformational probability distribution becomes narrowly peaked around a straight rod. Since a straight rod of given contour length has a finite hydrodynamic radius $R_{H}^{\max }$, this value serves as the curves' respective supremum. We independently calculated $R_{H}$ for straight rods (horizontal lines in figure 5.3) and can confirm this limiting behavior. The convergence is slower for longer chains because of entropic effects - more bending angles have to simultaneously be zero to reach the limit. Moreover, the limit is reached faster for smaller individual bead sizes $a$ because the Rotne-Prager matrices are functions of powers of $a / r$ ( $r$ representing the inter-bead distances), and are thus less sensitive to deviations from a perfectly straight rod for smaller $a$. We remark that in the absence of hydrodynamic interactions, $R_{H}$ would not depend on $\sigma$ at all: All chains would obey $R_{H}=a \cdot N$, independently of their conformation and of the conformational probability distribution which $\sigma$ tunes. From this perspective, we can understand the faster convergence of $R_{H}(\sigma)$ for smaller $a$ as being closer to the absence of hydrodynamic interactions in which $R_{H}(\sigma)=$ const. .

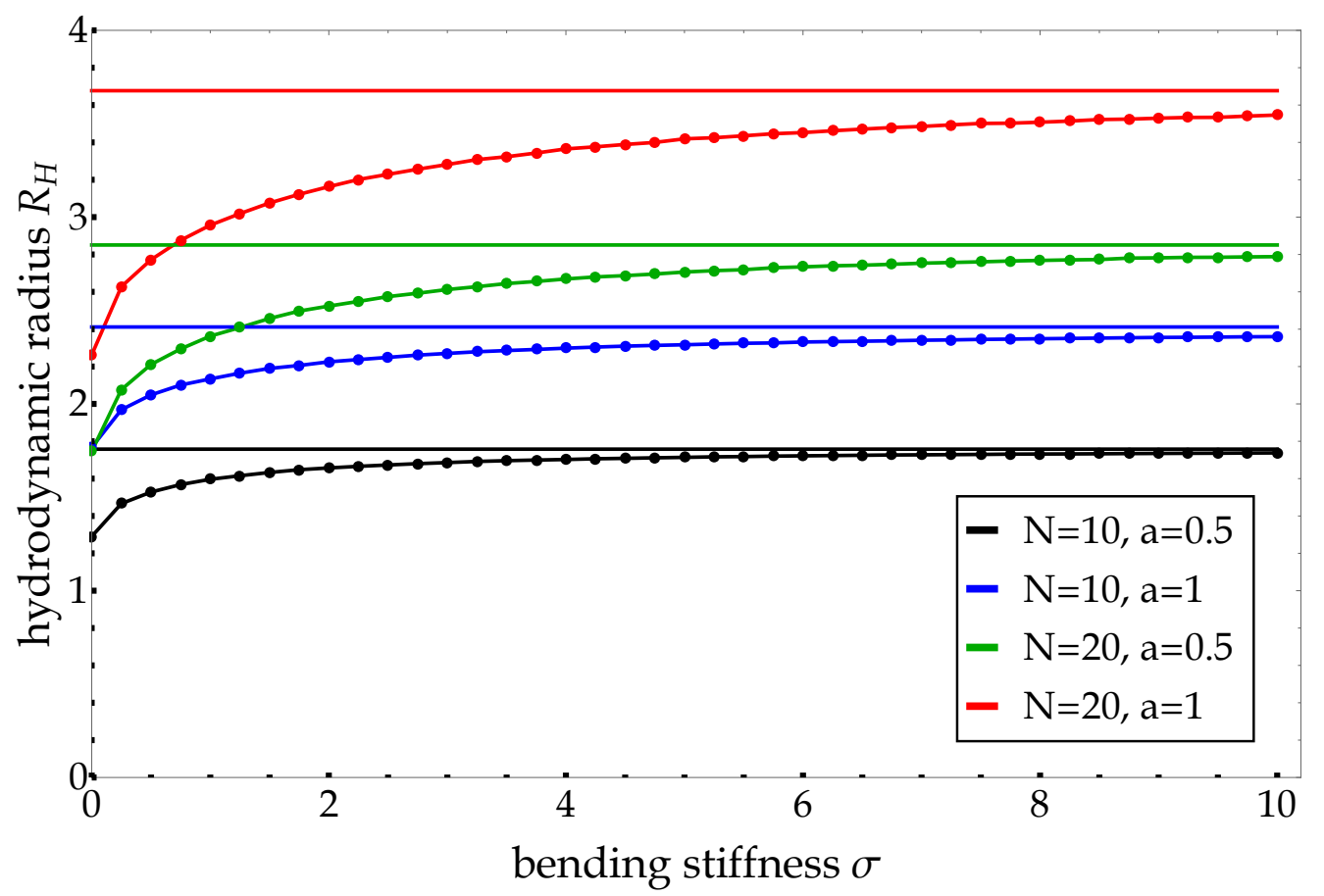

Figure 5.3: The hydrodynamic radius $R_{H}$ of model chains as a function of the chain's bending stiffness $\sigma$. The curves for $N=10$ are shown in black ( $a=0.5)$ and blue $(a=1)$. The curves for $N=20$ are shown in green $(a=0.5)$ and red $(a=1)$. For growing chain stiffness, all curves converge towards the hydrodynamic radius of the respective straight rod. These limiting values are shown as horizontal lines. 


\subsubsection{Contact rate}

While the hydrodynamic radius can be quickly calculated using MC sampling (seconds to minutes), the contact rate requires a full Langevin simulation (hours to days). For the sake of well-converged contact rate values, we thus only studied short model chains of length $N=10$ to demonstrate the influence of $\sigma$ and $a$ on the end-to-end contact rate $k_{+}$. Additionally, we demonstrate the so-called tail effect by comparing the end-to-end contact rate with the contact rate between the chain's end and a bead placed at different sites within the chain. We set the critical contact radius to $R_{C}=5.4 / 3.8 \approx 1.42$ because this is the same nondimensional radius that we later use for interpreting PET-FCS measurements.

The functional behavior of $k_{+}(N)$ is the treated in the next section which constitutes the main project of this thesis.

Contact rate as a function of bead size We must be careful when comparing contact rates from simulations with different values of $a$ for the following reason. The contact rate is the inverse of the mean first passage time $\langle\tau\rangle$ and as such, when reconverted into physical units, is proportional to the time scale $t_{0} \propto a$, which may differ between simulations. Without hydrodynamic interactions, for instance, the bead radius $a$ does not influence the numerics at all, and thus $k_{+} \propto a^{-1}$. To take this into account, we do not study $k_{+}$but the dimensionless quantity $k_{+} \cdot t_{0}$ as a function of $a$. In the absence of hydrodynamic interactions, the resulting curves would be constant. The influence of hydrodynamic interactions on the contact rate can thus be observed in the form of deviations from a horizontal line - a decreasing function means that hydrodynamic interactions lower the contact rate, and a growing function means that they enhance it. Thereby, we disentangle the effects that a larger bead (i) diffuses more slowly on its own, but (ii) is hydrodynamically coupled to other beads more strongly which dampens their relative motion. 
Figure 5.4 shows the functional form of $k_{+}(a)$ (normalized to the first data point for visual purposes) for $\sigma \in\{0,1\}$ and $N=10$. We observe that the curves are decreasing for both tested chain stiffnesses. Thus, the hydrodynamic coupling between the beads reduces the contact rate their relative movement is dampened because they fluctuate more cooperatively. Furthermore, the curve of the more flexible chain drops faster. Mathematically, that is because the limit of the Rotne-Prager matrices becoming identity matrices is reached faster for smaller inter-bead distances. Physically, the closer the beads' centers are, the more the beads overlap. Thus their relative movement is dampened faster in flexible chains as the beads grow.

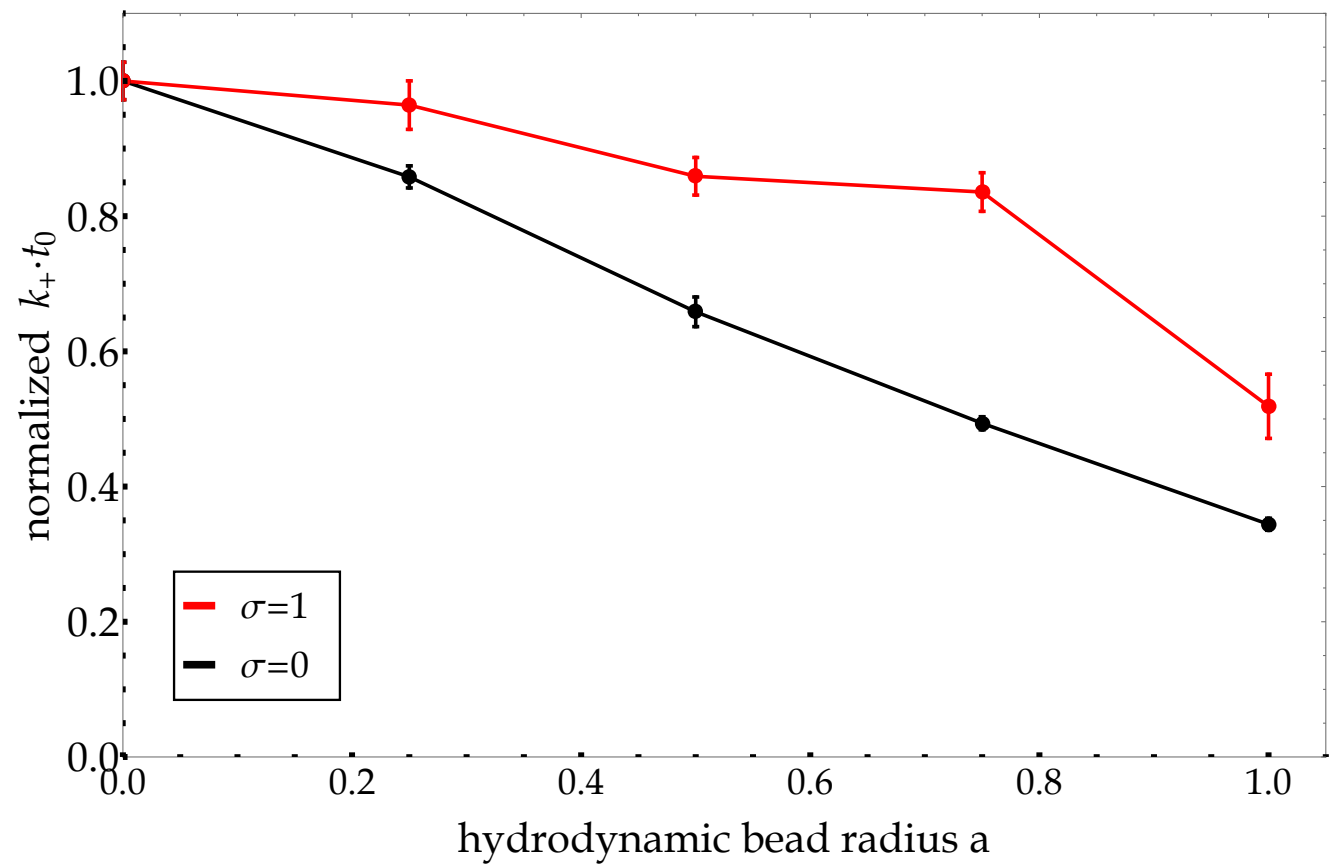

Figure 5.4: The simulated nondimensional contact rates $k_{+} \cdot t_{0}$ as a function of the hydrodynamic bead radius $a$, shown for two different bending stiffness values $\sigma$. The two curves were normalized such that both start at 1 . They both decrease, meaning that hydrodynamic coupling decreases the contact rate. The stiffer chain shows a slower decrease than the more flexible one. 
Contact rate as a function of bending stiffness Figure 5.5 shows the functional form of $k_{+}(\sigma)$ (normalized to the first data point for visual purposes) for $a \in\{0,1\}$ and $N=10$. We observe that bending stiffness decreases the simulated contact rate, which is intuitive - the energetic barrier which must be overcome for the chain ends to meet limits the rate of successful loop formation. Moreover, the curve for chains with smaller bead size drops faster. That is because hydrodynamic interactions decrease the contact rate, and the bending stiffness in turn reduces hydrodynamic interactions because the inter-bead distances grow the stiffer the chain is. As expected, the contact rate seems to vanish in the limit $\sigma \rightarrow \infty$.

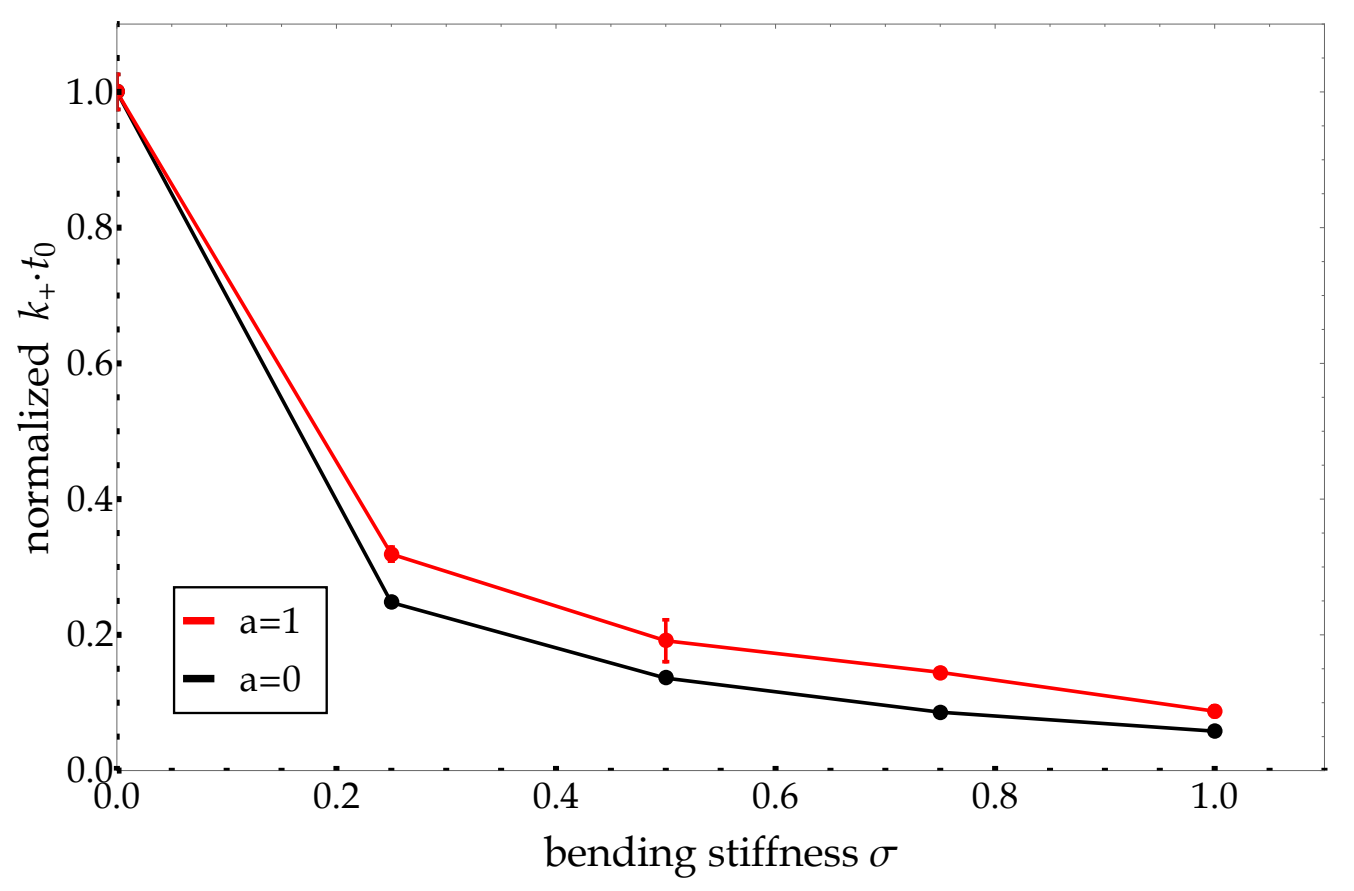

Figure 5.5: The simulated contact rates $k_{+}$as a function of the bending stiffness $\sigma$, shown for two different bead sizes. The chains consist of 10 beads. The curves were normalized such that they start at 0 in order to be visually comparable. The curve of the chain consisting of smaller beads drops faster.

Contact rate as a function of quencher position In figure 5.6, we show the simulated contact rate of two chains consisting of $N=10$ and $N=20$ beads respectively, both of which have parameters $a=\sigma=1$. The contact rate was evaluated for looping events taking place between the first bead (fluorophore) and another bead (quencher) at position $s$ within the chain, with the rest of the chain being present behind it. For growing $s$, entropic effects can be expected to lower $k_{+}$ as the vector connecting fluorophore and quencher grows, but "searches" for a small volume of fixed size $\propto R_{C}^{3}$. For $s \approx l_{p} \approx 2$, the (bending) energetic barrier which must be overcome for loop formation limits $k_{+}$but since $R_{C} \approx 1.42$ is finite, we do not expect to observe this effect here. 
We indeed observe that the contact rate decreases as the fluorophore-quencher $s$ distance increases. This decrease flattens out towards the ends of the respective chains: The growing $s$ is being balanced by the fact that the end of the chain can diffuse more freely than the middle of the chain. This is because for the chain end's diffusive dynamics, fewer beads have to be moved through the fluid. This reduces hydrodynamic friction and thus speeds up the dynamics. This "free-end effect" is not present in the $N=20$ chain at $s=10$ - it has additional beads behind the quencher, which is called the tail. The so-called tail effect is thus that it slows down the dynamics between fluorophore and quencher, reducing the contact rate. It cannot be inferred from the chain's conformational probability distributions. For instance, the random walk described by MC sampling samples the Boltzmann distribution but does not include any tail effect - the variations of bond angles in the middle of the chain occur completely independently of those at the chain's ends. In the Langevin simulation, however, correct physical dynamics are implemented: the hydrodynamics obey the Stokes equation which alone implies the tail effect. This is further investigated in the next section and discussed in chapter 7 .

Note that the tail effect has not been explicitly built into the model but emerges naturally!

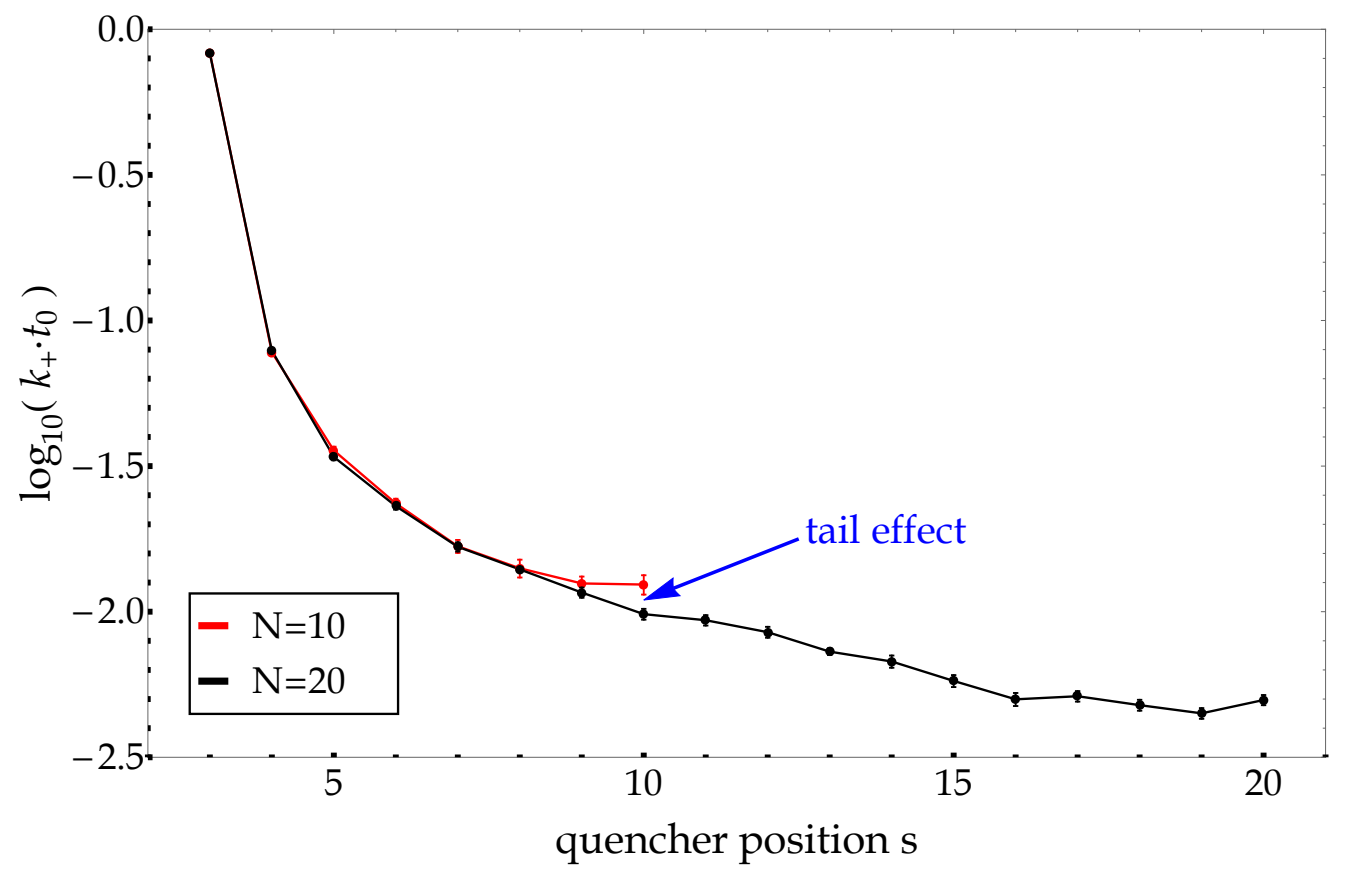

Figure 5.6: The simulated contact rate as a function of the distance $s$ between fluorophore and quencher. The fluorophore is the first bead of the chain, and the quencher's position $s$ is altered while the total length of the chain remains fixed. We compare the curves $k_{+}(s)$ for two chains of different length but otherwise identical parameters $a=\sigma=1$. The curves initially coincide, but towards their respective ends, they flatten out instead of dropping further. Hence, at $s=10$, the curve for $N=20$ lies below the curve for $N=10$ by around $21 \%$. This is called the "tail effect". 


\subsection{End-to-end contact rates and hydrodynamic radii of GS-repeats}

The project presented in this section constitutes the main project of this thesis. It was published in Physical Review E in November 2019 [129]. What follows are major parts of the published manuscript, which were have altered only slightly in order to fit into the narrative of this thesis. The experiments were performed by my colleagues Man Zhou (who also published some of the experimental results in his doctoral thesis [130]) and Arindam Ghosh, and here we omit the experimental details because they can be found in the published article, and the concepts were explained in section 2.5 (page 70).

In this project, we use PET-FCS and 2fFCS for measuring end-to-end contact rates $k_{+}$and hydrodynamic radius values $R_{H}$ of intrinsically disordered dye-labeled GS-repeats as a function of chain length. Additionally, we also investigate the tail effect in protein conformational fluctuation: The effect the chain beyond the quencher has on the contact rate. These are the measured proteins:

$$
\begin{array}{ll} 
& \text { Atto655-(GS })_{n}-\mathrm{W} \\
\text { and } \quad \text { Atto655-(GS) })_{5}-\mathrm{W}-(\mathrm{GS})_{5}
\end{array}
$$

where $n \in\{5,7,9,10,15,20\}$ and "G", "S" and "W" stand for the amino acids glycine, serine and tryptophan (see figure 1.1, page 6), and Atto655 is the fluorophore used, which is quenched by tryptophan. Thus the shortest proteins contain 11 , and the longest 41 amino acids.

Experiments The experimental results are summarized in table 5.1, and are also displayed in figures 5.9 and 5.15. The PET-FCS curves from which the contact rates were extracted are shown in figure 5.7 .

\begin{tabular}{c|ccccccc}
\hline $\mathrm{N}$ & 12 & 12 +tail & 16 & 20 & 22 & 32 & 42 \\
\hline$R_{H} / \AA$ & 9.4 & - & 9.6 & 10.1 & 11.1 & 13.2 & 15.2 \\
$k_{+} \cdot 10^{-6} s$ & 36.4 & 23.7 & 26.4 & 19.1 & 16.6 & 5.8 & 4.0 \\
$k_{-} \cdot 10^{-6} s$ & 12.8 & 9.2 & 11.4 & 11.2 & 11.0 & 12.4 & 9.9 \\
\hline
\end{tabular}

Table 5.1: The results of PET-FCS and 2fFCS measurements performed on GS-repeats of different chain lengths $N$. Error bars are shown in figures 5.9 and 5.15.

As expected, the hydrodynamic radius $R_{H}$ increases with chain length length, while the contact rate $k_{+}$decreases with chain length. The relation between contour length $L$ (see equation 5.3) and contact rate is well approximated by a power law with exponent $\epsilon=-1.86 \pm 0.15$, i.e.

$$
k_{+}(L) \propto L^{\epsilon} \quad .
$$




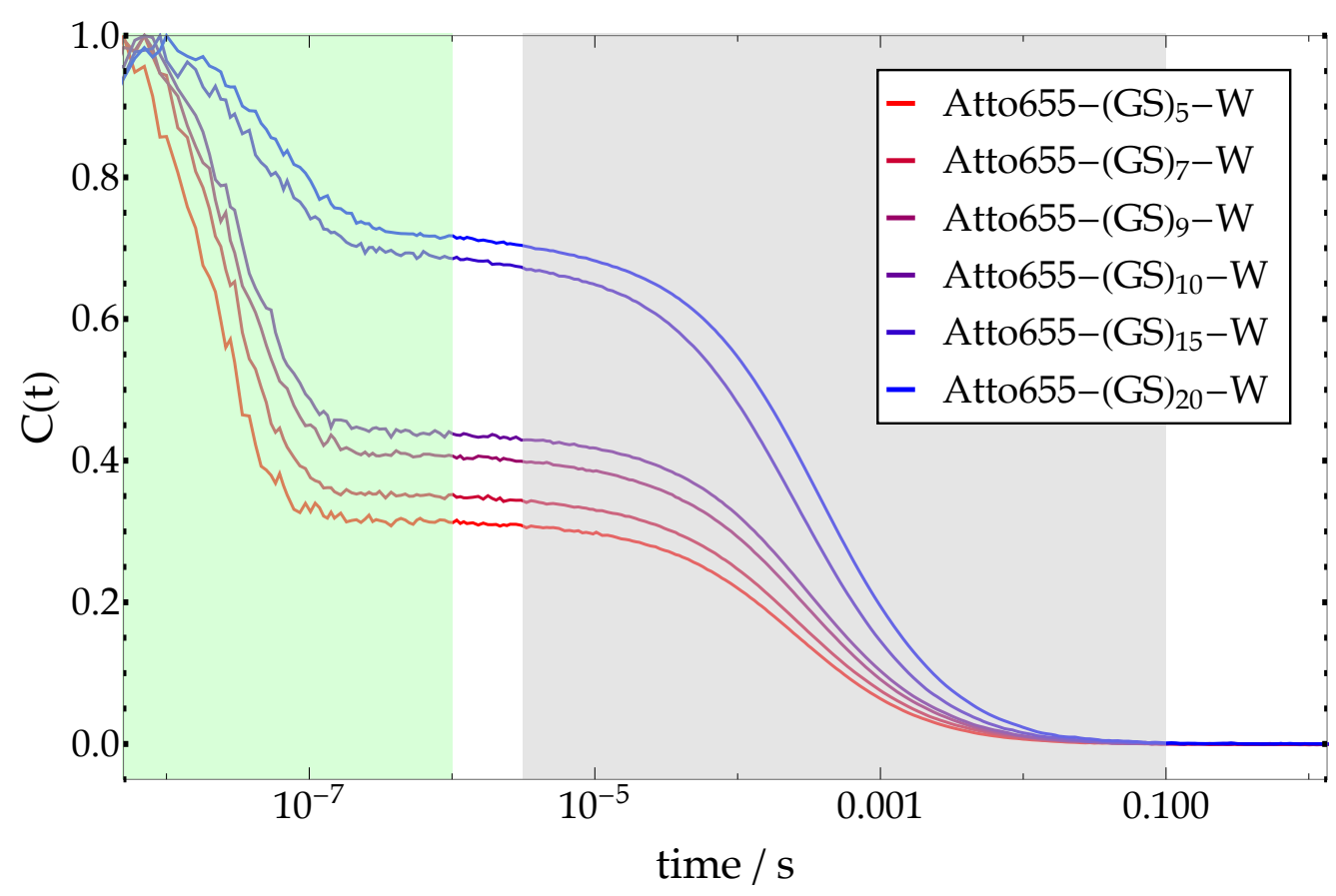

Figure 5.7: Normalized PET-FCS autocorrelation curves $C(t)$ (see equation 2.193, page 75) measured on different protein-dye constructs as indicated in the legend. The nanosecond timescale decay (green shading) is due to the opening/closing dynamics of the protein chain. The millisecond timescale decay (gray shading) is due to translational diffusion.

Mean loop formation times range from about 25 to $200 \mathrm{~ns}$, depending on chain length. From the power law fit of contact rates as a function of chain length, we estimate systematic contact rate errors as the average deviation between fit and experimental value, and find a value of around $12 \%$. A similar analysis for the hydrodynamic radius yields a value of $\sim 2.5 \%$, where an additional offset of $6 \AA$ was added to the power law to take the fluorophore into account. The error bars shown in figures 5.9 and 5.15 do further include experimental statistical errors which are comparatively small. Fitting a two-state-model (equation 2.193, page 75 ) to the experimental PET-FCS curves (see figure 5.7) delivers not only the closing rate $k_{+}$, but also the opening rate $k_{-}$. The latter is only used to weigh the open and closed states when calculating hydrodynamic radii (see equation 5.4 and figure 5.9). We find that the proteins (increasingly ordered by chain length) spend around 74, 70,63, 60, 32 and $29 \%$ of their time in the closed state.

To quantify the tail effect, we define the value $\zeta$ as the percentage by which the contact rate decreases due to the presence of the tail, i.e.

$$
\zeta:=1-k_{+}^{\text {tail }} / k_{+}
$$

Experimentally, we find $\zeta=34.9 \pm 12.2 \%$, meaning that the tail increases the mean time of contact formation by around one half. 
Protein model The molecules are modeled as semiflexible inextensible chains of $N$ connected spherical beads. Both hydrodynamic interactions as well as excluded volume effects are taken into account. Each bead represents one amino acid (pseudoatom) and has a fixed distance to its neighbors along the chain's backbone. We call this distance the bond length $d$ and set it to $d=3.8 \AA$, the distance between two $C_{\alpha}$-atoms in a protein's backbone. An additional bead representing the dye Atto655 is (semiflexibly) attached to one end of the chain with a fixed bond length of $d_{1}=7 \AA$. A model chain with $N$ beads ( $N-1$ amino acids) thus has contour length

$$
L=7 \AA+(N-2) \cdot 3.8 \AA
$$

The dye's hydrodynamic radius is known to be $a_{1}=6 \AA$ [131]. To account for the fact that a real chain cannot cross through itself, we assign an excluded-volume radius $R_{\mathrm{EV}}$ to each bead and reject all time steps that result in an overlap of two beads. Geometrically, this radius value has to be at least equal to $R_{\mathrm{EV}}=\sqrt{2} / 4 \cdot d$. The values $a_{1}, d_{1}, d, R_{\mathrm{EV}}$ and $R_{C}=5.4 \AA$ are fixed throughout this study and are not considered to be free parameters. This model allows us to simulate contact rate values using the constrained Langevin equation, and to calculate hydrodynamic radii using MC sampling. In all simulations, we set the temperature to room temperature $T=293.15 \mathrm{~K}$ and the value of viscosity to that of water, $\eta=0.955 \cdot 10^{-3} \mathrm{~Pa} \cdot \mathrm{s}[131]$.

A sketch of the model chain is provided in figure 5.8 (see also chapter 3 ). The two free model parameters are (i) the hydrodynamic radius $a$ of a single bead (amino acid), and (ii) the chain's bending stiffness $\sigma$ (or equivalently its persistence length $l_{P}$ ). It should be emphasized that our empirical model admits different values for the hydrodynamic and the excluded volume radius. The first one is related to geometric constraints of motion, whereas the second refers to hydrodynamic interactions within the protein. It should also be noted that the parameter $\sigma$ fixes the statistical properties of the chain, while the other parameter $(a)$ influences only its dynamical properties. The core tasks are to find values for these two parameters which globally match the experiments as best as possible, and to investigate how they influence the simulated dynamics.

Persistence length On page 68, it was described how the discrete bending stiffness $\sigma$ can be translated into the persistence length $l_{P}$. The described procedure is performed without the fluorophore attached to the chain because we are interested in the properties of the bare protein itself. The resulting value for $l_{P}$ turn out to be virtually independent of chain size, so we choose the shortest considered chain (11 beads) for the best convergence during MC sampling. For the later on considered discrete values of bending stiffness $\sigma=0, \ldots, 4 \AA$, the found persistence length values are $l_{P}=2.67,4.26,5.59,6.81$ and $7.97 \AA$. The finite persistence length of the chain with zero bending stiffness is purely due to excluded volume interactions. 
$R_{C}=0.54 \mathrm{~nm}$
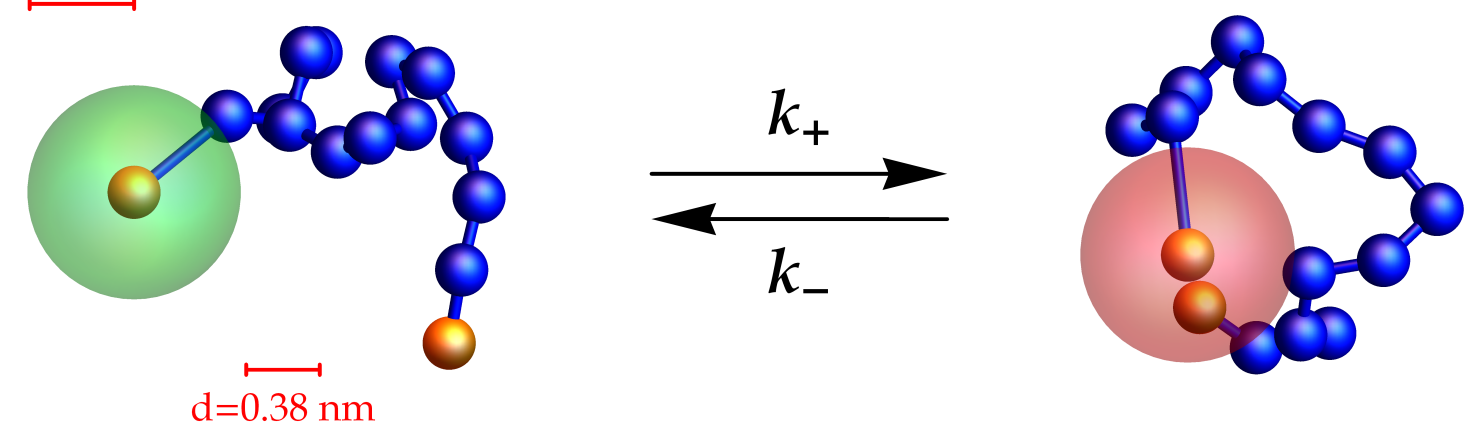

Figure 5.8: Schematic representation of the model molecule. Each blue bead represents one amino acid. The first and last bead represent the dye Atto655 (surrounded by the green/red sphere) and the quencher amino acid tryptophan, respectively. When the distance between their centers becomes smaller than $R_{C}=5.4 \AA$ (indicated by the green sphere turning red), fluorescence is completely quenched, otherwise completely non-quenched. The shown bead size is the excluded volume size, and throughout this entire study its radius is set to $\sqrt{2} / 4$ times the bond length - the minimum value preventing bond crossings. The hydrodynamic radius of a bead (not shown) may differ from its excluded volume radius. The hydrodynamic radius of Atto655 is set to $6 \AA$ [131], while all other beads (amino acids) are assumed to have the same hydrodynamic radius $a$ (free model parameter). The length of one bond (rods connecting adjacent beads) is fixed to $d=3.8 \AA$. The fluorophore Atto655 is attached to the chain's end at a fixed distance of $7 \AA$.

Contact rate A contact event between the fluorophore and tryptophan is assumed to take place whenever the distance $r$ between their centers becomes smaller than a critical value $R_{C}$. Conformations with $r<R_{C}$ are considered a closed state, whereas those with $r>R_{C}$ are considered an open state. Under equilibrium conditions, transitions from the open to the closed state occur with an average rate $k_{+}$, and transitions back to the open state with an average rate $k_{-}$. One experimental complication is that the direct contact between the fluorophore and the tryptophan has a non-negligible association energy which stabilizes the closed state. In our model, a chain almost immediately returns to the open (fluorescent) state after a diffusive encounter between the fluorophore and the quencher. Thus, the model-derived opening rate $k_{-}$is expected to be much larger than the experimentally observed one. Consequently, we will focus here on the rate $k_{+}$which is assumed to be unaffected by a short-ranged association energy. The critical radius $R_{C}$ and resulting contact rate $k_{+}$depend on the photophysical details of contact-induced fluorescence quenching. Here, we adopt a value of $R_{C}=5.4 \AA$ following the experimental study of reference [117]. The value that would be most appropriate for our model calculations is difficult to pin down since we replace amino acids and the fluorophore by spheres which do not interact energetically. This may lead to some systematic bias of the absolute values of the model-derived contact rates, but their dependence on chain length and model parameters should be unaffected. 
For chain length values of $N \in\{12,16,20,22,32,42\}$ (number of amino acids plus one fluorophore bead), we computed $k \in\{4,6,8,8,14,20\}$ independent trajectories for each tested parameter set, respectively. Initial conditions were drawn from a Boltzmann distribution by running a sufficiently long MC simulation ( $10^{6}$ steps) before the start of each Brownian dynamics simulation. Simulation times per trajectory ranged from $10^{4} t_{0}$ to $10^{5} t_{0}$, which is on the order of tens of microseconds. The resulting statistical errors for the determined contact rate values were between $1.3 \%$ and $5.7 \%$.

Hydrodynamic radius In the experiments, fluorophore and quencher temporarily stick together after loop formation (due to the non-zero contact interaction energy), which alters the average chain size and the equilibrium ensemble. Because our model does not include this effect, the simulated conformational ensemble $\Omega^{\text {sim }}$ will not correctly reproduce the experimentally sampled ensemble $\Omega^{\exp }$ because conformations belonging to the closed state are underrepresented in it. To correct for this, we divide the equilibrium ensemble $\Omega^{\text {sim }}$ into two sub-ensembles $\Omega^{\text {open }}$ and $\Omega^{\text {close }}$, defined by the distance between fluorophore and quencher exceeding (or being less than) the critical radius $R_{C}=5.4 \AA$ for contact formation. A weighted average over both sub-ensembles then yields the corrected diffusion coefficient

$$
D^{\text {model }}=\sum_{\alpha \in\{\text { open,close }\}} p_{\alpha} \cdot\langle D\rangle_{\alpha}
$$

In numerical practice, open and closed states sampled during an MC sampling procedure contribute to the two averaging processes independently. The probabilities $p_{\text {open }}$ and $p_{\text {close }}$ are calculated from the chain-length dependent experimental ratio $k_{-} / k_{+}=p_{\text {open }} / p_{\text {close }}$ (see page 75 ). Finally, the hydrodynamic radius of a chain is given by $R_{H}=k_{B} T /\left(6 \pi \eta D^{\text {model }}\right)$. Example $R_{H^{-}}$ values calculated with this method for chains of different lengths but identical parameters $(\sigma, a)$ are presented in figure 5.9 together with the experimental values.

Comparison with simulations In what follows, we explain how we determined optimum parameters of hydrodynamic bead radius $a$ and bending stiffness $\sigma$ that globally fit all experimental findings best - simultaneously for all chain lengths. Note that, as we saw in the previous section, both parameters influence $k_{+}$and $R_{H}$.

Since computation time for the hydrodynamic radius is much shorter than for simulating contact rates, we first determined values for $a$ which lead to the best match between calculated and measured $R_{H}$ values. We did this for five discrete values of bending stiffness $\sigma$ from 0 to $4 \AA$ in steps of $1 \AA$. For each of them, we determined the best value $a(\sigma)$ by minimizing the quadratic relative deviations from the experimental $R_{H}$ values summed over all chain lengths:

$$
a(\sigma)=\operatorname{argmin} \sum_{N \in \text { chain lengths }}\left(\frac{R_{H}^{\text {experiment }}(N)-R_{H}^{\text {model }}(a \mid \sigma, N)}{R_{H}^{\text {experiment }}(N)}\right)^{2} .
$$




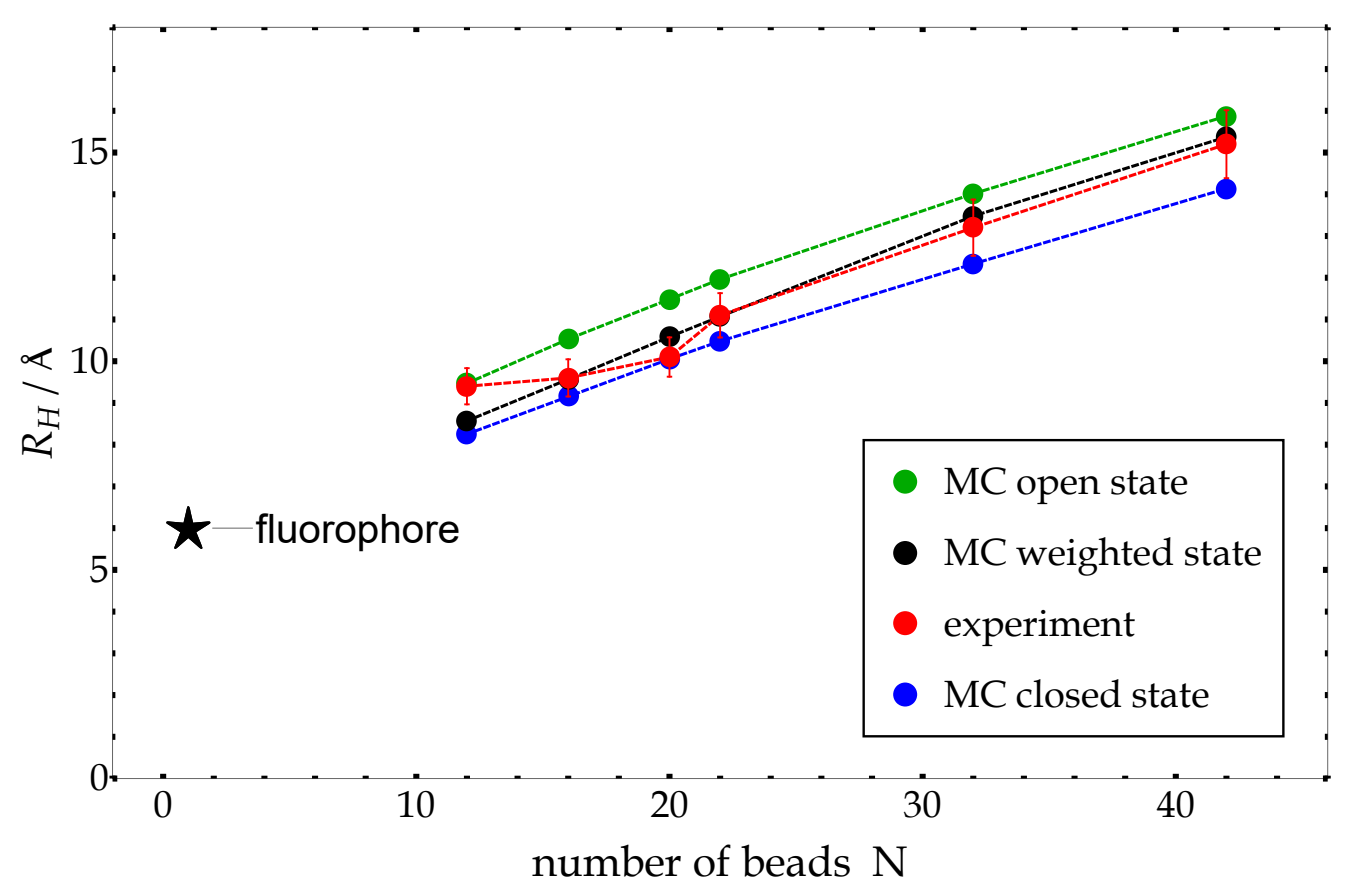

Figure 5.9: Experimental (red) and model (black) hydrodynamic radius values as functions of chain length. MC results for $R_{H}$ were calculated using equation 4.23 (see page 96), considering the sub-ensembles of open (green) and closed (blue) states separately. The black curve was calculated by weighting open and closed states according to their experimentally measured ratio $k_{-} / k_{+}=p_{\text {open }} / p_{\text {close }}$. The shown model curve has parameters $\sigma=1 \AA$ (persistence length $4.26 \AA$ ) and the corresponding best matching bead radius $a(\sigma=1 \AA)=3.81 \AA$ (see equation 5.5). The hydrodynamic radius of the attached fluorophore was set to $6 \AA$ [131] (single data point on the left). Model curves for the other candidate parameter sets $(\sigma, a)$ (figure 5.10) look very similar and are not shown.

Thus, we obtained five candidate parameter sets $[\sigma, a(\sigma)]$. Figure 5.9 compares the experimental and best model curves $R_{H}(N)$ for one of these five parameter sets. The dependency of $a$ on $\sigma$ is shown in figure 5.10 and the candidate parameter values are also summarized in table 5.2.

Next, we performed Brownian dynamics simulations for all chain lengths $N$ and all five candidate parameter sets. The five simulated curves $k_{+}(L)$ are shown in figure 5.11. Each of them follows a power law virtually precisely. Observing that the slope in the log-log plot changes systematically as the parameter set is changed, we fit power laws to the functions $k_{+}(L)$ for each candidate parameter set. The so-found exponent values $\epsilon$ are shown in figure 5.12 and table 5.2. All simulation values for the rates $k_{+}$are of the same order of magnitude as the experimental ones, but are systematically too large. In particular, we find for the studied $\sigma$-values of 0 to $4 \AA$ that the simulated values of the contact rates exceed the experimentally measured ones by $35,32,23,19$ and $8 \%$ (averaged over $N$ ). Considering the uncertainties of the simulation results due to the not precisely known correct value of contact radius $R_{C}$, these results are highly encouraging. 


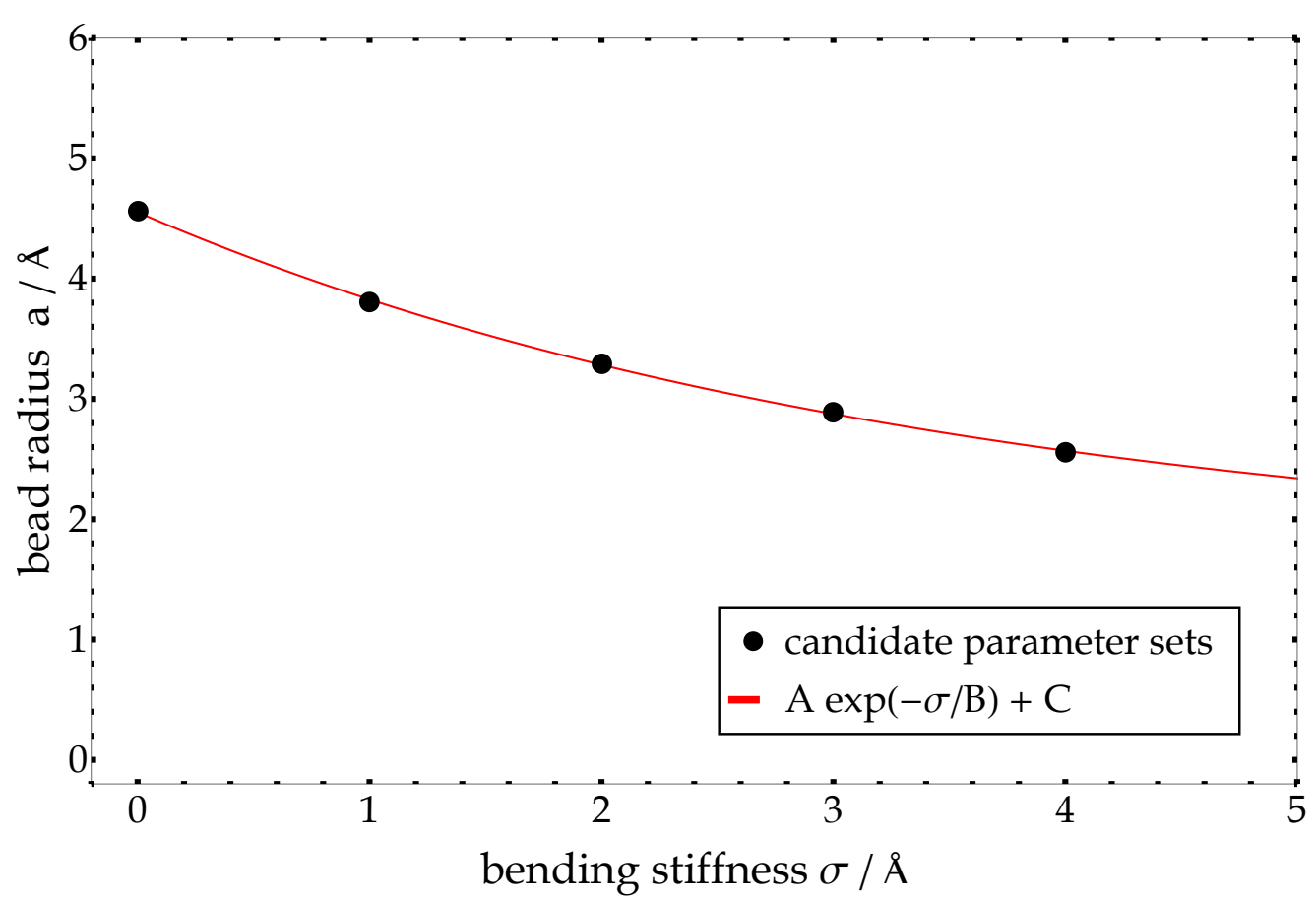

Figure 5.10: The "candidate parameter sets" for which Brownian dynamics simulations were run. For five discrete values of $\sigma(\sigma=0, \ldots, 4 \AA)$, values of $a$ were determined that lead to hydrodynamic radius values that match the experimental values best. This procedure yields tuples $(\sigma, a)$ (shown as black dots), which can be fitted with the heuristic function $a=A \exp (-\sigma / B)+C$ where $A=2.90 \AA, B=3.47 \AA$ and $C=1.65 \AA$ (shown in red).

At the same time, identifying one parameter set as the best-fitting one based on a least-squares criterion is problematic. As an alternative, we study two dimensionless quantities that can be extracted from both simulated and experimental contact rates. The first one is the exponent $\epsilon$, which quantifies the functional form of $k_{+}(\mathrm{L})$ rather than its magnitude, and the second is the tail effect value $\zeta$ (equation 5.2). Both are invariant to rescaling all rates by a constant factor and may hence be expected to depend only weakly on photophysical details of contact formation. In contrast, the rates' values themselves do. The power law exponent $\epsilon$ increases along the candidate parameter curve (figure 5.10 ) from $\sim-2.1$ to $\sim-1.7$, seemingly converging towards $\sim-1.5$ (see figure 5.12). We attribute this to the fact that excluded volume effects are less influential for chains with a higher persistence length, which changes the scaling behavior of their end-to-end distance as a function of chain length (see discussion, page 142). Tail effect values $\zeta$ weakly increase along the candidate parameter curve from $\sim 35 \%$ to $\sim 42 \%$, see figure 5.13 . All these tail effect values are well within the experimental error margin of $34.9 \pm 12.2 \%$. 


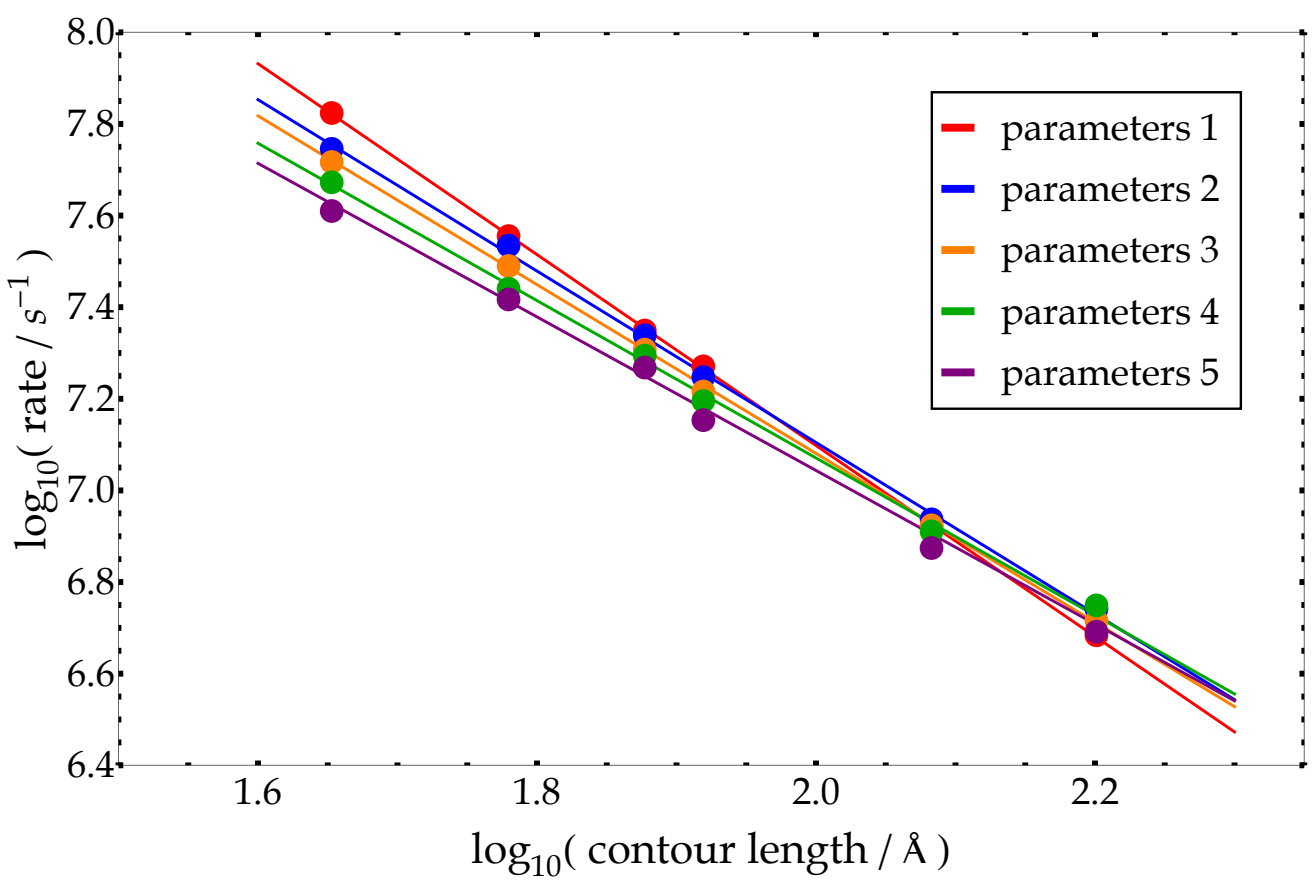

Figure 5.11: The simulated contact rates for all candidate parameter sets as functions of contour length $L$. We show the data points together with respective power law fits (lines) to the simulated curves. The slopes (power law exponents) change systematically as the parameter set is changed, see also figure 5.12 and table 5.2.

\begin{tabular}{c|ccccc}
\hline set & 1 & 2 & 3 & 4 & 5 \\
\hline \hline$\sigma / \AA$ & 0 & 1 & 2 & 3 & 4 \\
$\Leftrightarrow l_{P} / \AA$ & 2.67 & 4.26 & 5.59 & 6.81 & 7.97 \\
\hline$a / \AA$ & 4.56 & 3.81 & 3.29 & 2.89 & 2.56 \\
\hline \hline exponent $\epsilon$ & -2.08 & -1.87 & -1.84 & -1.72 & -1.68 \\
\hline tail effect $\zeta / \%$ & 35.5 & 32.8 & 37.6 & 38.4 & 41.9 \\
\hline \hline probability p & 0.33 & 0.98 & 0.97 & 0.63 & 0.42 \\
\hline
\end{tabular}

Table 5.2: Simulation results for power law exponent $\epsilon$ and tail effect value $\zeta$ for the investigated candidate parameter sets $(\sigma, a)$. The corresponding persistence length values and the probability density values calculated with equation 5.6 are also given.

The globally best-fitting parameter set is determined as follows. We assume that the experimentally derived joined probability distribution $p(\epsilon, \zeta)$ for $\epsilon$ and $\zeta$ is given by the following product of two Gaussian distributions:

$$
p(\epsilon, \zeta)=\frac{1}{Z} \exp \left[-\frac{\left(\epsilon-\mu_{\epsilon}\right)^{2}}{2 \delta_{\epsilon}^{2}}-\frac{\left(\zeta-\mu_{\zeta}\right)^{2}}{2 \delta_{\zeta}^{2}}\right]
$$




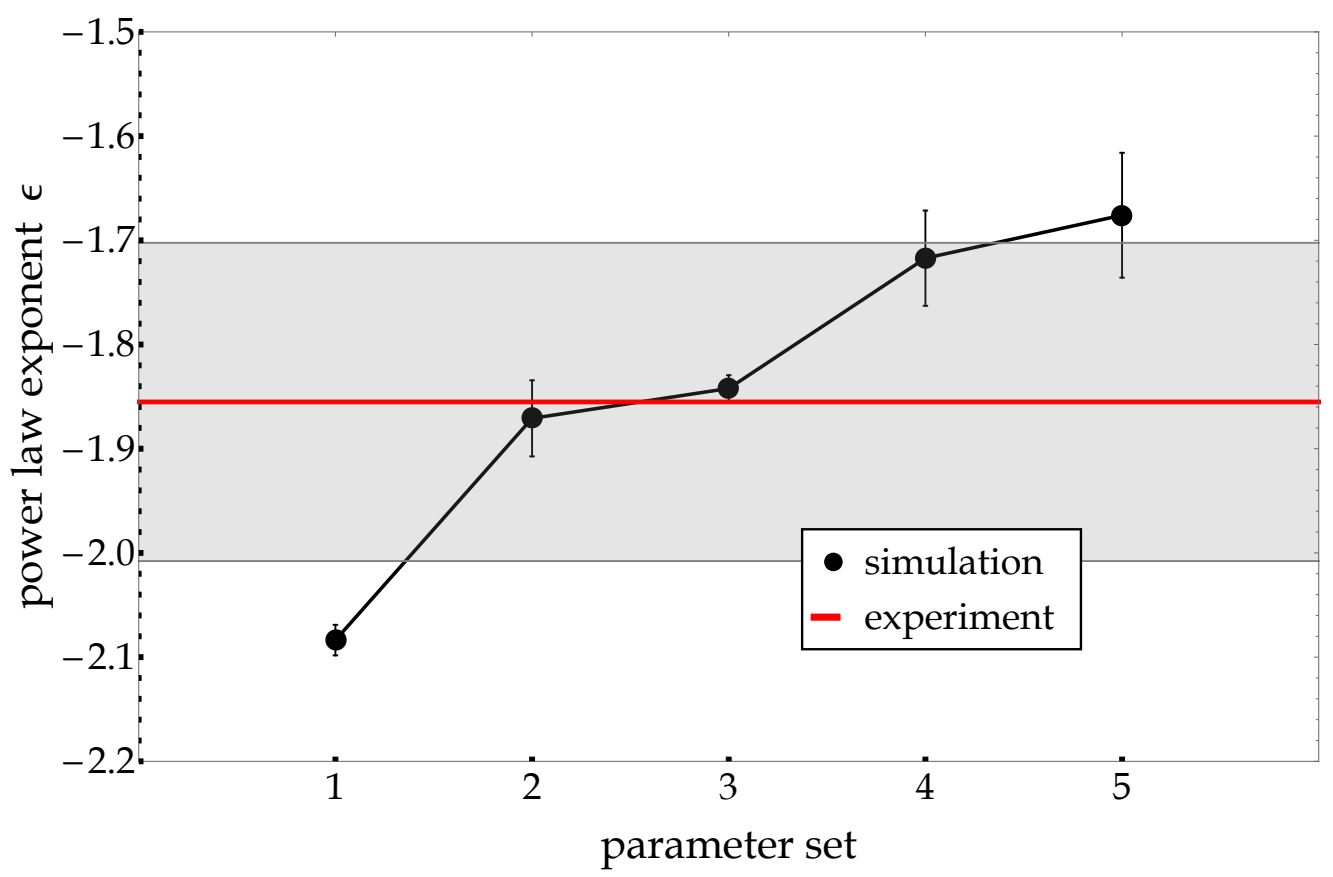

Figure 5.12: For each candidate parameter set, the simulated end-to-end contact rates as a function of contour length were fitted with a power law. The resulting power law exponents are shown here as a function of the parameter set $(\sigma, a)$ (see figure 5.10 and table 5.2). The exponent values increase monotonically, seemingly converging towards a value of $\sim-1.5$, similar to the scaling behavior found for ideal chains as predicted by SSS theory [19]. We attribute this to the fact that for increasingly flexible chains (from right to left), excluded volume interactions play a more important role, which alters their scaling behavior (see discussion, page 142). The power law exponent for the experimental data is shown in red $(-1.86 \pm 0.15)$. The shaded area is its fit error.

with the mean values $\mu_{\epsilon}, \mu_{\zeta}$ and standard deviations $\sigma_{\epsilon}, \sigma_{\zeta}$ determined from the experimental measurements, and $Z$ being a normalizing factor. This allows us to associate with each simulation result a probability density value. For the investigated candidate parameter values of $(\sigma, a)$, the resulting probability density values are shown in figure 5.14 and table 5.2, where the figure shows two separate probability density curves, one as a function of persistence length $l_{P}$ and the other as a function of hydrodynamic bead radius $a$. By fitting these curves with Gaussian functions, parameter values that globally best match the experiments are determined. The found values are $a=3.5 \pm 0.7 \AA$ and $l_{P}=5.2 \pm 1.9 \AA$, which is the main result of this project. We have simulated the contact rates for this parameter set, which is shown in figure 5.15 together with the experimental rates for comparison, which are on average about $17 \%$ smaller. 


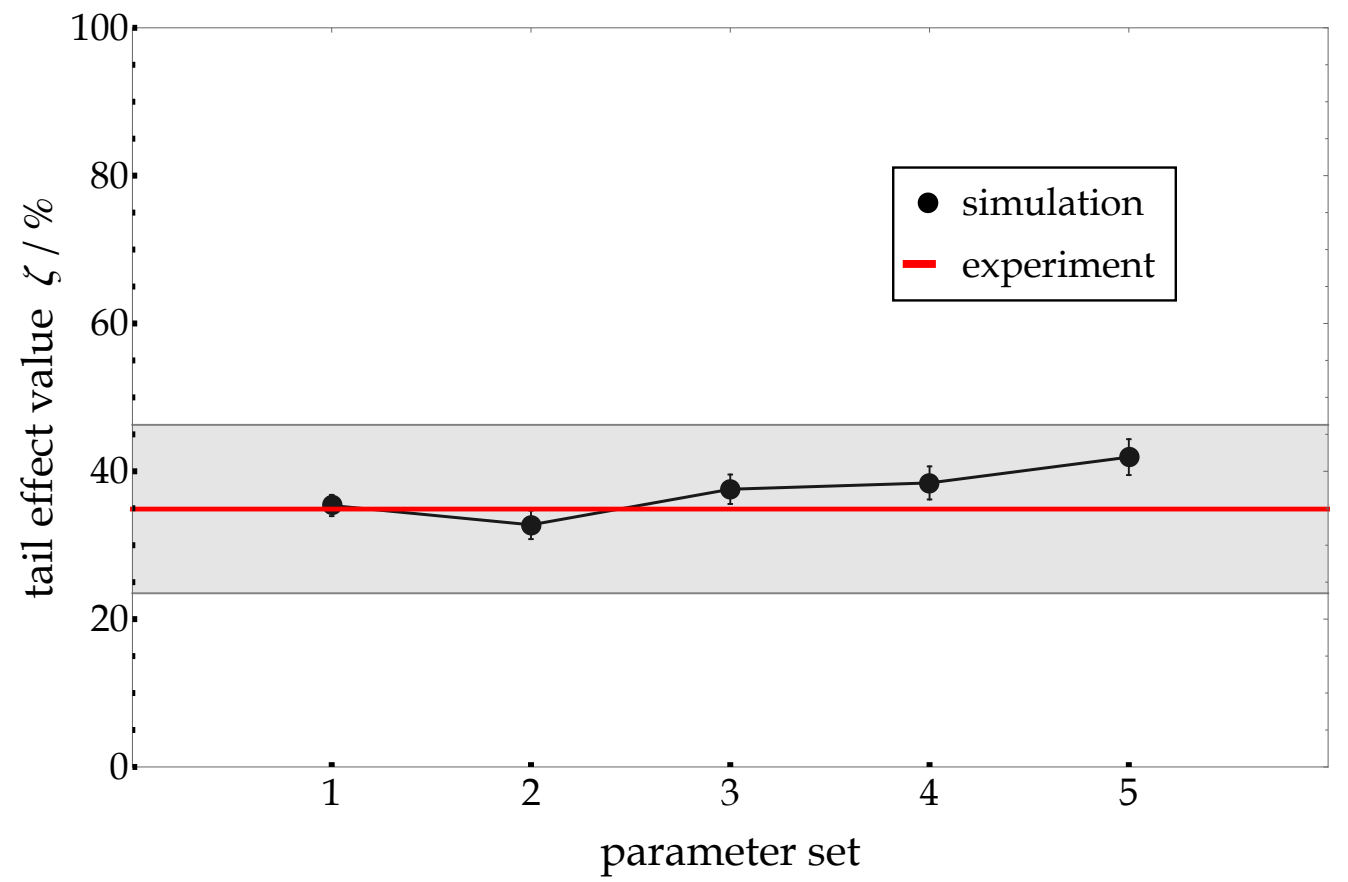

Figure 5.13: Tail effect values $\zeta$ for Atto655-(GS) $)_{5}$ W-(GS) $)_{5}$ versus Atto655-(GS) $)_{5}-\mathrm{W}$. Simulation values are shown as a function of the parameter set (figure 5.10 and table 5.2). The experimental value (34.9\%) is shown in red, and the shaded area indicates its error of $\sim 12.2 \%$.

Influence of model parameters on exponent and tail effect An important question is how changes in $\sigma$ and $a$ individually affect the values of $\epsilon$ and $\zeta$. For investigating this question, we performed additional simulations where we change $\sigma$ while $a$ is fixed and vice versa. Tables 5.3 and 5.4 show their outcome.

\begin{tabular}{ccc}
\hline \multicolumn{3}{c}{$a=3.81 \AA$} \\
\hline$l_{P} / \AA$ & tail effect $\zeta / \%$ & exponent $\epsilon$ \\
\hline 2.67 & $34.8 \pm 1.2$ & $-2.10 \pm 0.02$ \\
4.26 & $32.8 \pm 1.9$ & $-1.87 \pm 0.04$ \\
7.72 & $34.1 \pm 2.7$ & $-1.66 \pm 0.03$ \\
\hline
\end{tabular}

Table 5.3: Fixing the bead radius and increasing the chain stiffness increases the power law exponent, while the tail effect value remains constant within the statistical error.

Our results clearly show that $\epsilon$ is predominantly a function of chain stiffness but not of hydrodynamic coupling strength, while the $\zeta$ is predominantly a function of hydrodynamic coupling strength but not of chain stiffness. Thus $\epsilon$ and $\zeta$ can be varied independently which justifies our silent assumption in equation 5.6 that they are statistically independent. 


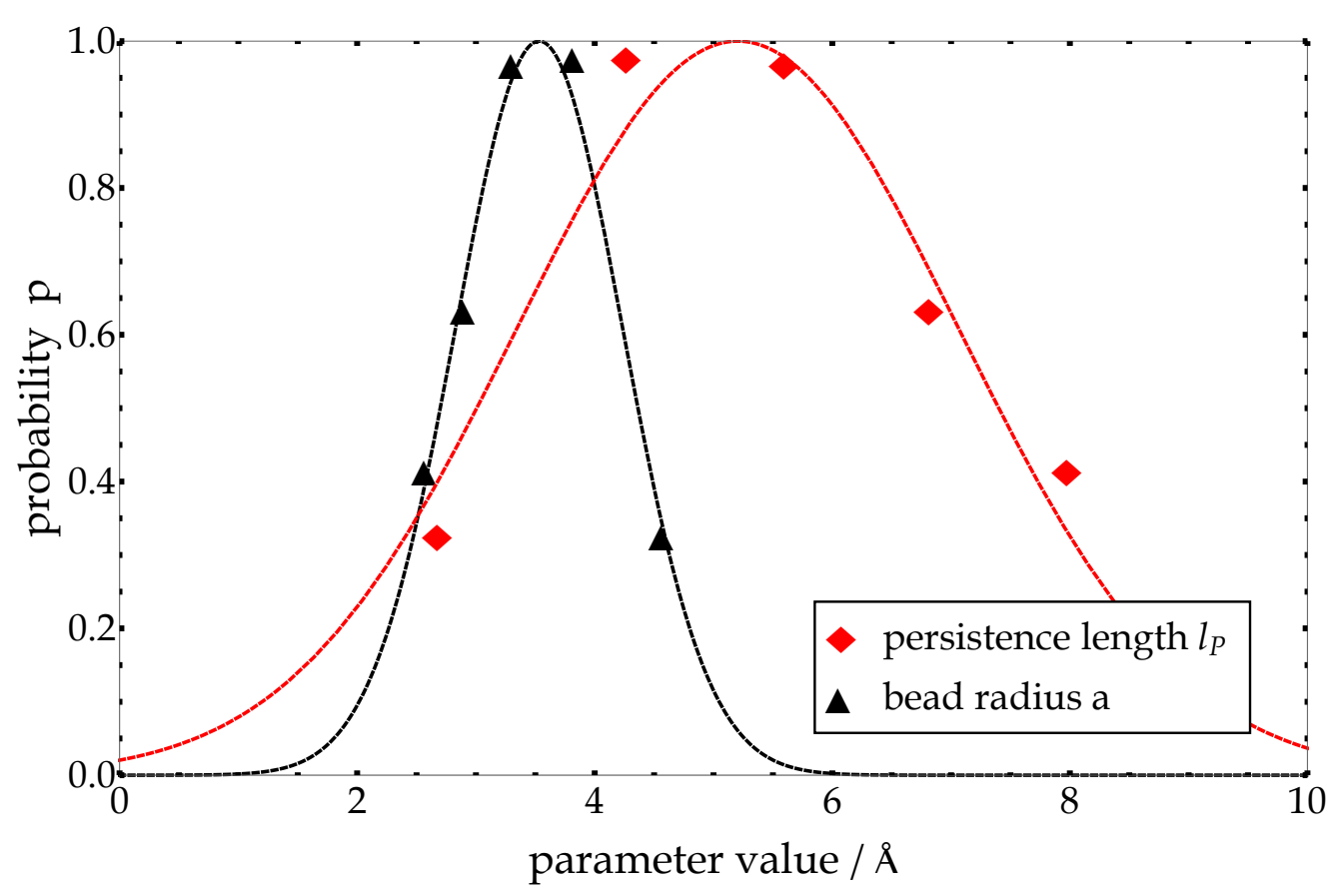

Figure 5.14: Probability density values (equation 5.6) as a function of hydrodynamic bead size $a$ (triangles) and persistence length $l_{P}$ (diamonds). These values were both fitted with Gaussian distributions (solid lines), and their maximum values and widths function as the optimal parameter values of $l_{P}$ and $a$ and their standard deviations. We find $l_{P}=5.2 \pm 1.9 \AA$ and $a=3.5 \pm 0.7 \AA$.

\begin{tabular}{ccc}
\hline \multicolumn{3}{c}{$l_{P}=4.26 \AA$} \\
\hline$a / \AA$ & tail effect $\zeta / \%$ & exponent $\epsilon$ \\
\hline 1.90 & $43.0 \pm 1.3$ & $-1.93 \pm 0.03$ \\
3.81 & $32.8 \pm 1.9$ & $-1.87 \pm 0.04$ \\
5.71 & $26.5 \pm 5.4$ & $-1.93 \pm 0.03$ \\
\hline
\end{tabular}

Table 5.4: Fixing the chain stiffness and increasing the bead radius decreases the tail effect value, while the power law exponent remains constant within the statistical error.

Finally, we have studied the question how much our results are influenced by the excluded volume effect. For this purpose, we repeated our simulations for candidate parameter set $2(a=3.81 \AA$ and $\sigma=1 \AA$ ), but not taking into account any excluded volume effects by setting the excluded volume radius $R_{\mathrm{EV}}$ to zero. The result (see table 5.5) shows that the value of the power law exponent $\epsilon$ dramatically increases. Moreover, the tail effect value is reduced, indicating that the tail's presence sterically reduces the chance of looping events. 


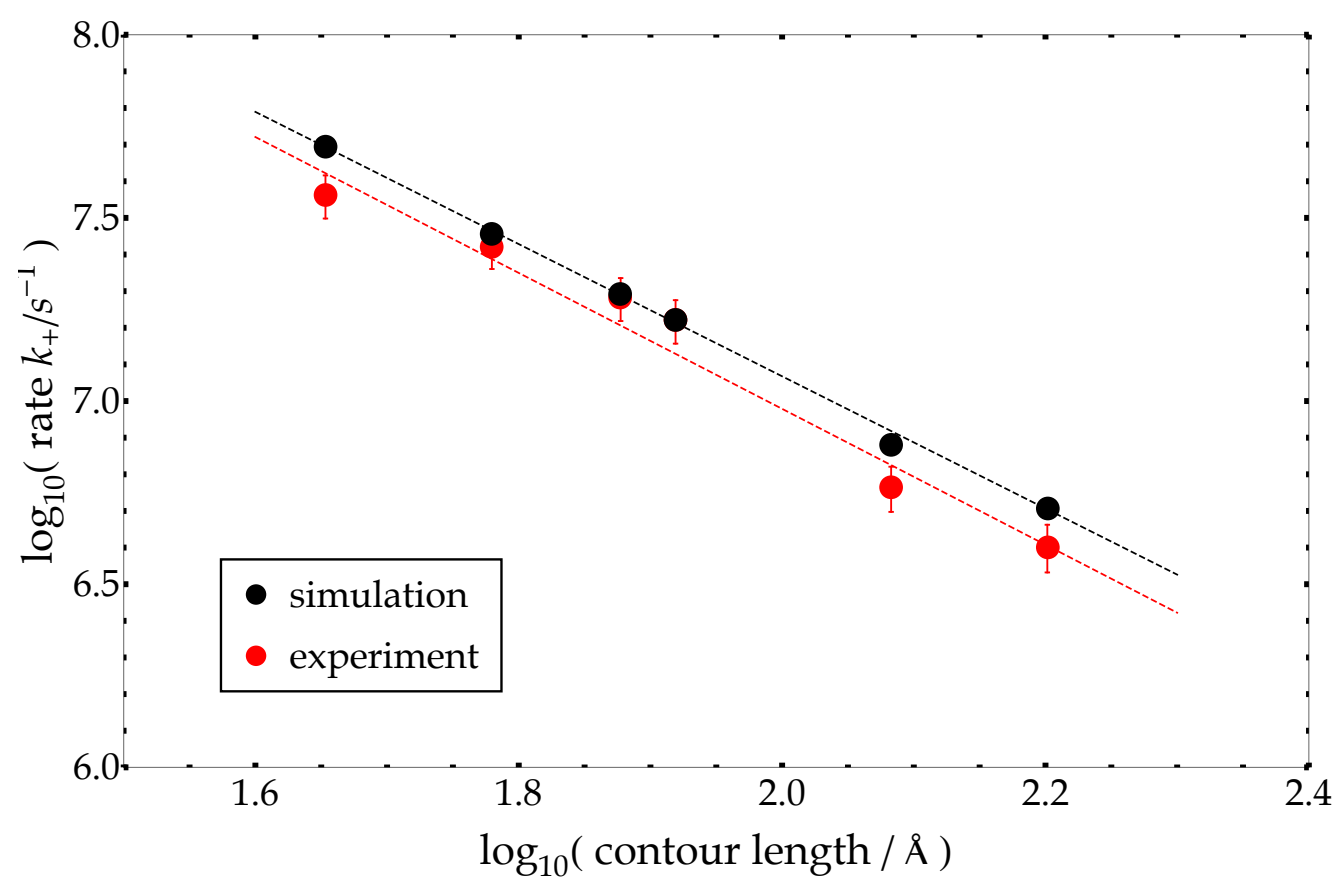

Figure 5.15: Simulated (black dots) and measured (red dots) end-to-end contact rates as function of contour length together with power law fits (dashed lines). Parameter values chosen for this figure are $a=3.53 \AA$ and $\sigma=1.83 \AA$, which corresponds to a persistence length of $5.2 \AA$. Simulated rates are $\sim 17 \%$ larger than experimentally measured values. This lies well within the expected inaccuracy due to the simplified model details of contact formation. Moreover, the found power law exponents for the experimental $(-1.86 \pm 0.16)$ and simulated $(-1.80 \pm 0.04)$ curves are in excellent agreement. The statistical errors of the simulated contact rates shown here are within a few percent $(\sim 3 \%)$ of the displayed values which is why the corresponding error bars in logarithmic space are smaller than the round symbols.

\begin{tabular}{ccc}
\hline \multicolumn{3}{c}{$\left(a, l_{P}\right)=(3.81,4.26) \AA$} \\
\hline$R_{\mathrm{EV}} / d$ & tail effect $\zeta / \%$ & exponent $\epsilon$ \\
\hline 0 & $25.3 \pm 1.9$ & $-1.53 \pm 0.01$ \\
$\sqrt{2} / 4$ & $32.8 \pm 1.9$ & $-1.87 \pm 0.04$ \\
\hline
\end{tabular}

Table 5.5: Turning off excluded volume effects decreases the tail effect and the power law exponent's magnitude. 
Best contact radius This paragraph contains extra information which cannot be found in the published manuscript [129]. Here, we investigate how the contact radius $R_{C}$ influences the simulated power law exponent $\epsilon$ and tail effect $\zeta$, and how the contact rates themselves depend on it. We employ the best fitting candidate parameter set ( $a \approx 3.53 \AA$ and $\sigma \approx 1.83 \AA$ ) and we keep track of contact events with contact radii $R_{C}=3.8 \AA, R_{C}=5.4 \AA$ and $R_{C}=7.6 \AA$ for all previously considered chain lengths $N . R_{C}=5.4 \AA$ is the previously used value and the two other values lie approximately a factor of $\sqrt{2}$ under and above it. The resulting mean first passage times in numerical units $t_{0}$ (inverse contact rates) can be found in table 5.6, and the corresponding power law exponents and tail effects can be found in table 5.7.

\begin{tabular}{c|ccccccc}
\hline & $N=12$ & $N=12+$ tail & $N=16$ & $N=20$ & $N=22$ & $N=32$ & $N=42$ \\
\hline$R_{C}=3.8 \AA$ & $170 \pm 3$ & $277 \pm 7$ & $280 \pm 5$ & $418 \pm 7$ & $480 \pm 8$ & $1007 \pm 35$ & $1509 \pm 62$ \\
$R_{C}=5.4 \AA$ & $90 \pm 3$ & $144 \pm 3$ & $155 \pm 4$ & $227 \pm 5$ & $267 \pm 5$ & $586 \pm 33$ & $871 \pm 33$ \\
$R_{C}=7.6 \AA$ & $45 \pm 1$ & $67 \pm 1$ & $77 \pm 1$ & $121 \pm 2$ & $140 \pm 3$ & $297 \pm 6$ & $485 \pm 17$ \\
\hline
\end{tabular}

Table 5.6: The simulated mean first passage times (in numerical units) for the globally best-fitting parameter set $a \approx 3.53 \AA$ and $\sigma \approx 1.83 \AA$. They are shown for all chain lengths and for three different critical contact radius values: the one used before (5.4 $)$ ), and two values approximately a factor of $\sqrt{2}$ above and below that value.

\begin{tabular}{c|ccc}
\hline & $R_{C}=3.8 \AA$ & $R_{C}=5.4 \AA$ & $R_{C}=7.6 \AA$ \\
\hline power law exponent & $-1.750 \pm 0.031$ & $-1.790 \pm 0.024$ & $-1.885 \pm 0.017$ \\
tail effect & $38.8 \pm 1.9$ & $37.0 \pm 1.6$ & $33.0 \pm 1.2$ \\
\hline
\end{tabular}

Table 5.7: The power law exponents and tail effect values corresponding to the mean first passage times in table 5.6.

The mean first passage times are approximately a factor of 2 apart for each increase/decrease in $R_{C}$ by a factor of $\sqrt{2}$. We conclude that we have at hand a scaling law which goes roughly $k_{+} \propto R_{C}^{2}$. At the same time, the experimental rates lie about $17 \%$ below the simulated ones. The "true" contact radius $R_{C}^{\text {true }}$ that should be used such that model and experiment show the same contact rate values is hence

$$
R_{C}^{\text {true }}=\frac{5.4 \AA}{\sqrt{1.17}} \approx 5 \AA
$$

Linearly interpolating the simulated data from table 5.7 to this value, one obtains a power law exponent which is about $0.7 \%$ larger than the one we found by using $R_{C}=5.4 \AA$, and a tail effect which is about $1.2 \%$ larger. These deviations are small when compared to the error bars of the experimental values, which are about $8.1 \%$ and $12.2 \%$ respectively. 
Conclusion We have measured end-to-end contact rates as well as hydrodynamic radius values of short GS-repeats, labeled with Atto655. As expected, the hydrodynamic radius $R_{H}$ increases with chain length from $0.9 \mathrm{~nm}$ to $1.5 \mathrm{~nm}$ for the considered range of chain lengths from 11 to 41 amino acids. In contrast, the contact rate $k_{+}$decreases with chain length. We also investigated the so-called tail effect by measuring and comparing contact rates of the proteins Atto655-(GS) 5 -W and Atto655-(GS) $)_{5}-\mathrm{W}-(\mathrm{GS})_{5}$, the latter having a "tail" behind the quencher position.

To interpret our experimental findings, we performed Brownian dynamics simulations of semiflexible bead-rod chains with an attached fluorophore, including hydrodynamic interactions and excluded volume effects. By comparing our simulations with our experimental results, we determined model parameter values that best fit all observations. In particular, we found for the hydrodynamic radius of one amino acid a value of $a=3.5 \pm 0.7 \AA$, and for the persistence length a value of $l_{P}=5.2 \pm 1.9 \AA$. Our model excellently reproduces all experimental hydrodynamic radius values, the exponent of the power law describing contact rate change as a function of chain length, and the effect of the tail. At the same time, it reproduces the contact rates' absolute values with surprising accuracy even though this match was not a fit criterion at all. Further testing of the model showed that the tail effect value is determined by the interplay of hydrodynamic drag and hydrodynamic interactions, and that the power law's exponent is determined by the interplay of persistence length and excluded volume radius.

We conclude that Brownian dynamics of generic bead-rod chains can be an efficient and valuable tool for describing a large set of experimentally measured characteristics of protein size (hydrodynamic radius) and dynamics (loop formation rates). This is even more remarkable as our model contains only two free and independent parameters. Moreover, we found that excluded volume effects are a crucial ingredient in modeling intrinsically disordered proteins. 


\subsection{Internal-to-end contact rates and hydrodynamic radii of FG-proteins}

This section presents a follow-up project to the previous one on GS-repeats and is thus largely analogous to the previous section. The difference is the following: Instead of GS-repeats with different chain lengths, here we investigate a different amino acid sequence with a single chain length $N=52$. It is given by

\section{Atto655-CTNNGGGFNLKTTNSNTSNNFLSSSGFTNGGATGNSKTQQASSSGFLSTGA}

and, other than the GS-repeats, is highly heterogeneous. The quencher tryptophan is not placed at the other chain end, but at different positions within the chain, which are marked in red. These marked amino acids are replaced by the quencher tryptophan one at a time such that a total of four internal-to-end contact rates are measured. In contrast to the previous section, the quencher position (let us call it $s$ ) now plays the role of chain length, and thus a power law is fitted to the function $k_{+}(s)$. Since there is only a single chain length, no tail effect is measured or simulated. For a given parameter set, all internal-to-end contact rates can be calculated from the same simulated trajectories - the four relevant inter-bead distances are simply kept track of simultaneously. The PET-FCS experiments were performed by Man Zhou (and published in his doctoral thesis [130]), and the 2fFCS experiments by Arindam Ghosh.

Experiments The experimental results are summarized in table 5.8.

\begin{tabular}{c|cccc}
\hline $\mathrm{s}$ & 13 & 23 & 33 & 43 \\
\hline$R_{H} / \AA$ & 17.6 & 17.9 & 19.2 & 19.2 \\
$k_{+} \cdot 10^{-6} s$ & 5.3 & 3.2 & 2.1 & 1.4 \\
$k_{-} \cdot 10^{-6} s$ & 4.3 & 4.1 & 4.8 & 4.7 \\
\hline
\end{tabular}

Table 5.8: The results of PET-FCS and 2fFCS measurements performed on FG-proteins, with the quencher tryptophan being placed at different sites $s$ within the chain. Error bars are shown in figures 5.16 and 5.18.

Hydrodynamic radius The model predicts (see figure 5.16) that the hydrodynamic radius of a closed chain is smaller when the quencher is positioned further away from the fluorophore. Intuitively, this makes sense - a chain's size is reduced more strongly when its ends are pulled together than when one end is pulled towards a center-position. Furthermore, the model predicts that the hydrodynamic radius of an open chain is not significantly altered as the quencher position changes. This also makes sense as the respective ensembles of open conformations are to a great extend identical. 
Meanwhile, the experiments show that the proteins spend more time in the open state the greater the dye-quencher distance is. This makes the model's weighted mean of open and closed states shift more towards the open state's hydrodynamic radius as $s$ increases. This effect counteracts the drop of hydrodynamic radii in the closed state and hence, the model's predicted hydrodynamic radius depends only weakly on the quencher position, which holds true for all candidate parameter sets. The experimental data, on the other hand, show an increase as a function of quencher position. This increase is weak, however, and only slightly exceeds the experimental error bars.

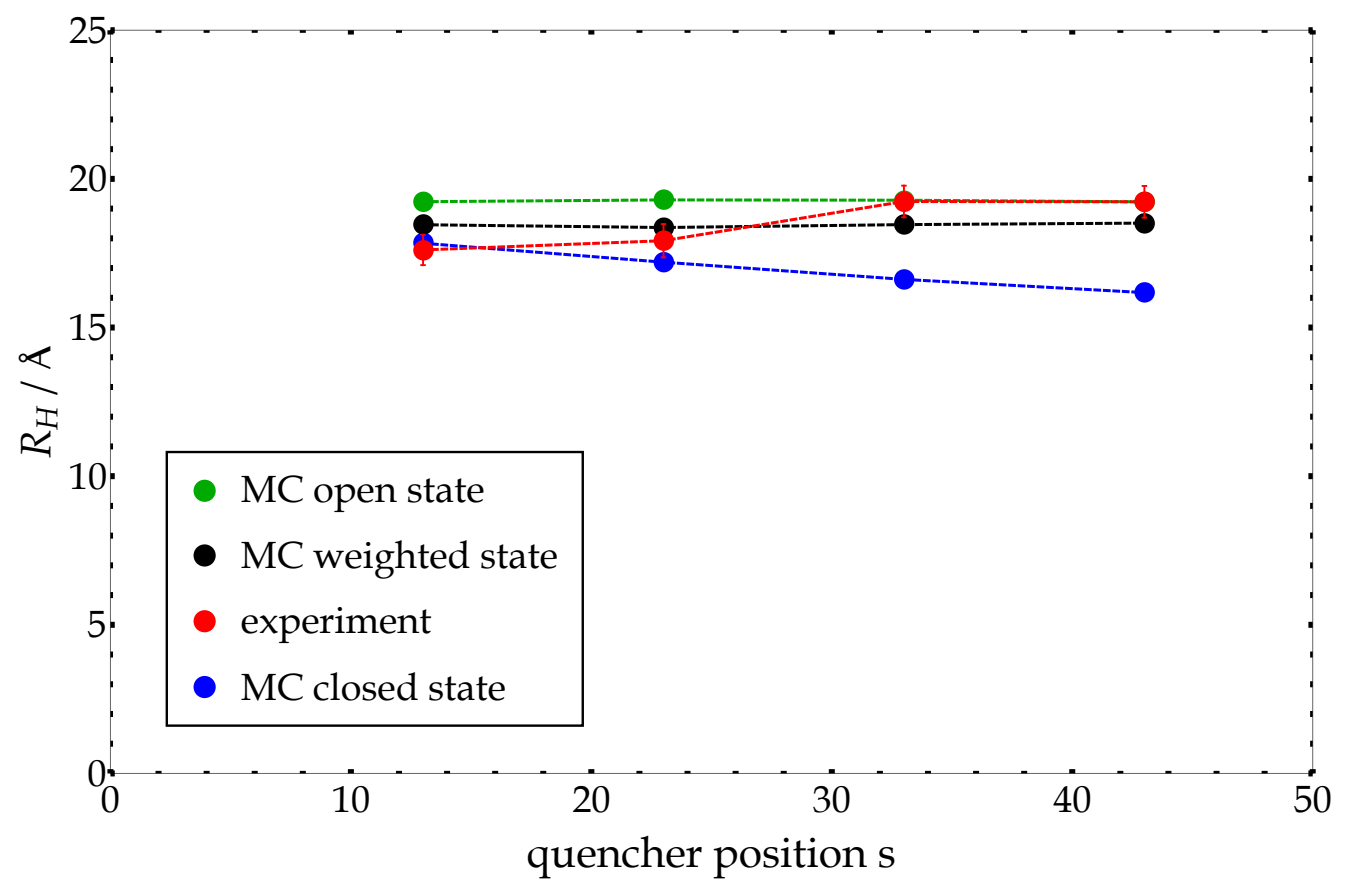

Figure 5.16: Experimental (red) and model (black) hydrodynamic radius values as functions of quencher position. MC results for $R_{H}$ were calculated using equation 4.23 (page 96), considering the sub-ensembles of open (green) and closed states (blue) separately. The red curve was calculated by weighting open and closed states according to their experimentally measured ratio $k_{-} / k_{+}=p_{\text {open }} / p_{\text {close }}$. Shown are model curves for $\sigma=3 \AA$ (persistence length $6.81 \AA$ ) and the best matching parameter $a(\sigma=3 \AA)=2.90 \AA$. Model curves for the other parameter sets $(\sigma, a)$ look similar and are not shown.

Candidate parameter sets Turning to the candidate parameter sets which best fit the experimental hydrodynamic radii (see figure 5.17), we observe a stronger dependence of $a$ on $\sigma$ than for the previously studied GS-repeats. This may be due to the fact that the FG-protein is longer and thus a change in persistence length entails a steeper growth of the effectively occupied volume by the chain, which must be compensated by lowering the bead radius $a$ further. 


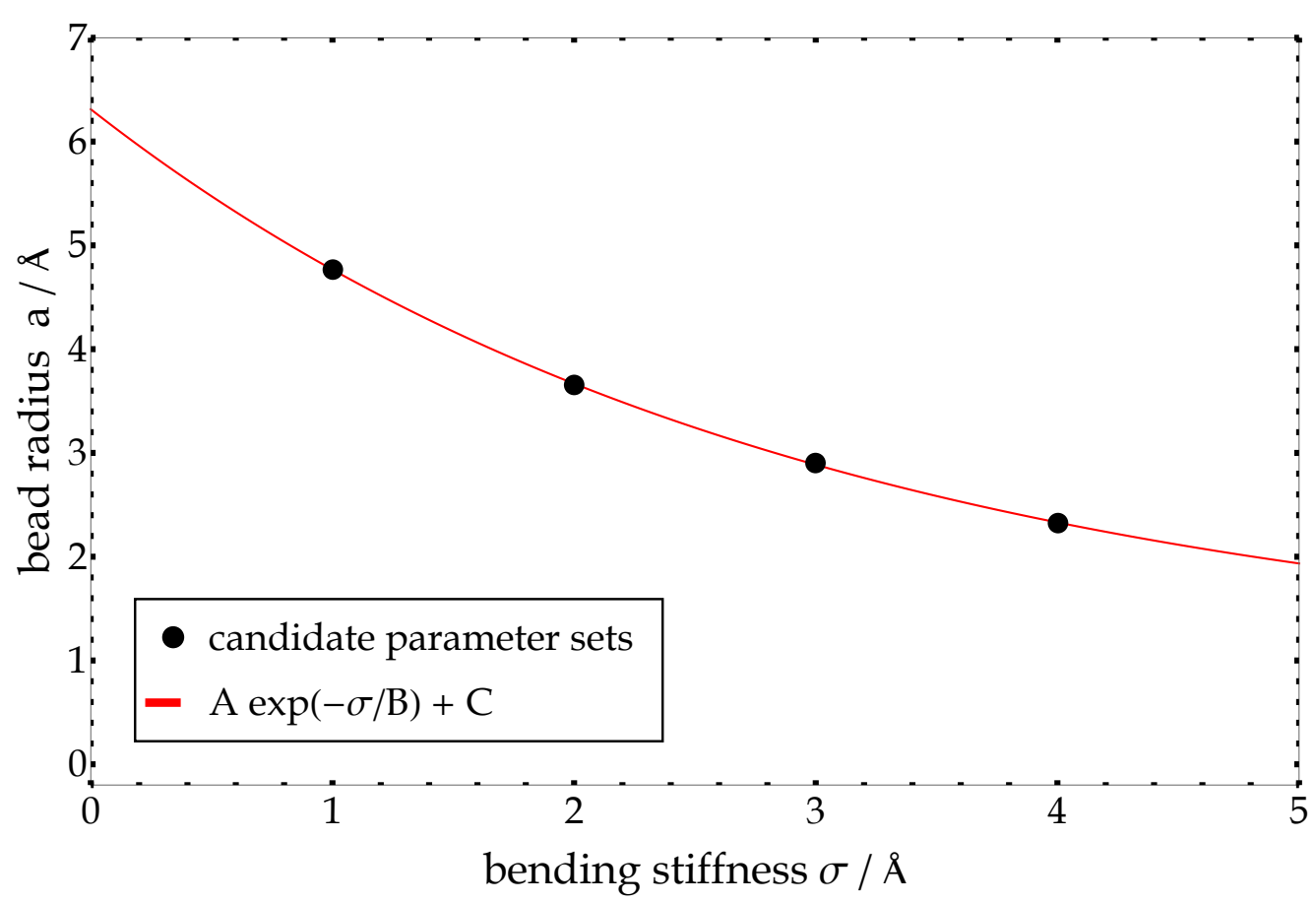

Figure 5.17: Parameter sets for which Brownian dynamics simulations were run. For four discrete values of $\sigma(\sigma=1, \ldots, 4 \AA)$, values of $a$ were determined that lead to hydrodynamic radius values that match best the experimental values, see figure 5.16. This procedure yields tuples $(\sigma, a)$ (shown as black dots) which can be fitted with the heuristic function (red line) $a=A \exp (-\sigma / B)+C$ where $A=5.35 \AA, B=2.93 \AA$ and $C=0.96 \AA$.

Contact rates The simulated contact rates as a function of quencher position are again wellfitted by power laws (see figure 5.18), and again the corresponding power law exponent changes systematically as a different candidate parameter set is chosen. However, the simulated rates' values do not reproduce the experimental values regardless of the chosen parameter set - they exceed the experimental ones by a factor of 2 to 5, which may be considered as acceptable considering the systematic uncertainty of simulated rates due to the chosen contact radius $R_{\mathrm{C}}=5.4 \AA$. However, this factor of 2 to 5 does not fit together with the one found for GS-repeats (a factor of $\approx 1.17$ ) and even more importantly, the experimental power law exponent differs significantly from all simulated ones.

Conclusion The model failed in reproducing the functional form of the measured internal-toend contact rates in FG-proteins. Possible causes are mainly the inhomogeneity of the amino acid sequence as well as the possible presence of internal friction. This will be discussed in the next chapter (see page 145). 

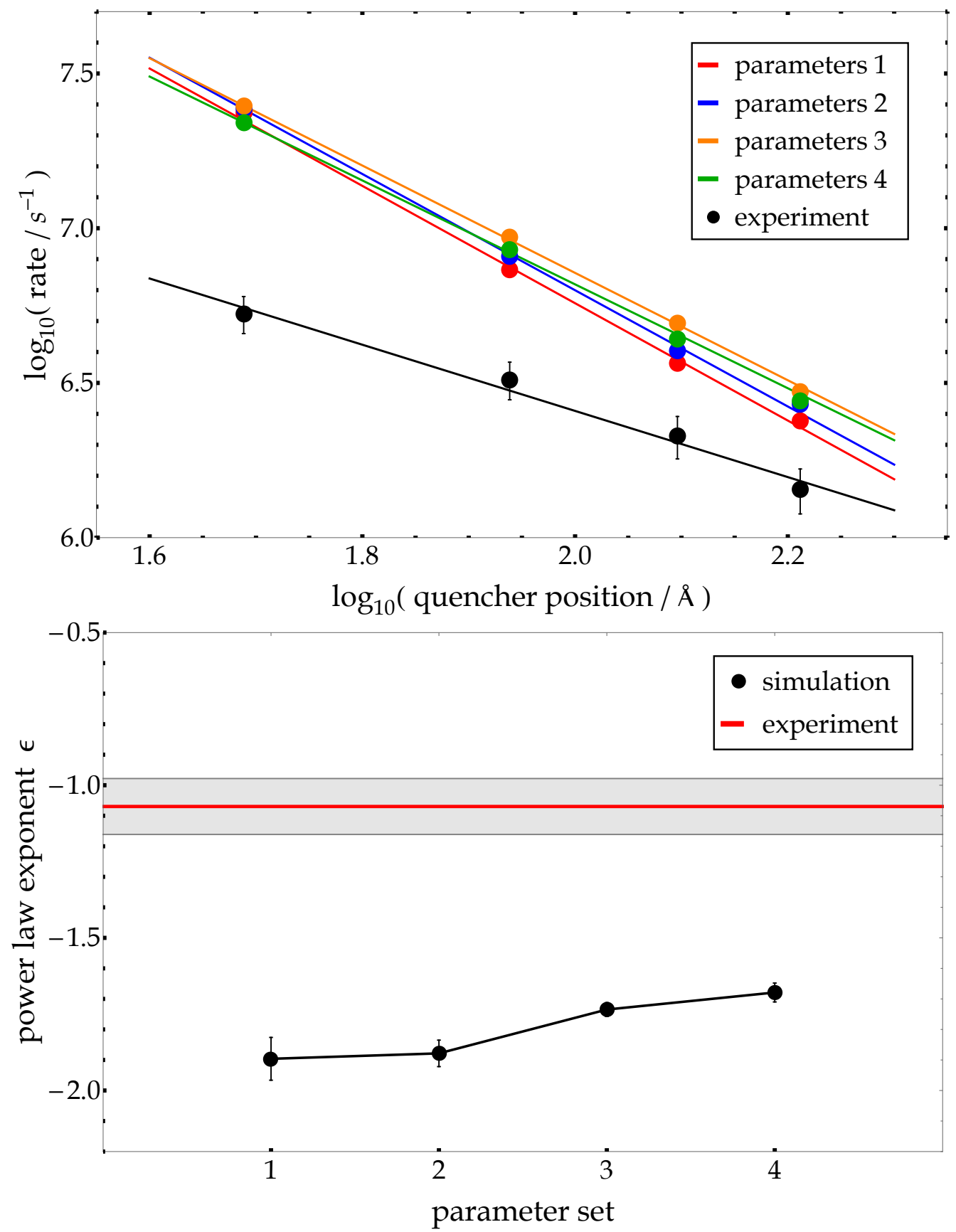

Figure 5.18: Top: The simulated contact rates for all chain lengths and candidate parameter sets, together with the experimentally measured contact rates (black dots). We show the data points together with respective power law fits (lines). Their slopes (power law exponents) change as the parameter set is changed and the experimental curve is notably flatter than all simulated ones. Furthermore, the measured rates are smaller than the simulated ones roughly by a factor of $2-5$. Bottom: For each candidate parameter set, simulated internal-toend contact rates as a function of the quencher position were fitted with a power law. The resulting power law exponents are shown here as a function of the candidate parameter set $(\sigma, a)$. As can be seen, exponent values increase monotonically, converging towards a value of $\sim-1.6$. The power law exponent for the experimental data is shown in red $(-1.07 \pm 0.09)$. The shaded area is its fit error. 


\subsection{Polymer dynamics using dynaMIET}

This project is yet (May 2020) unpublished due to unresolved experimental complications. The experimental work has been done by my colleagues Arindam Ghosh, Roman Tsukanov, Ingo Gregor, Narain Karedla, Sebastian Isbaner and Jan Christoph Thiele. In what follows, I present my own work in this project as a supplementary method for dynaMIET experiments in general. The particular system that we are working on merely serves as a hands-on application. Experimental details which are irrelevant in the context of this thesis are therefore omitted.

In this project, we do not study contact rates in freely swimming proteins using PET-FCS, but reconfiguration times of single-stranded DNA (ssDNA) consisting of 45 monomers (nucleotides) bound to a surface using dynaMIET (see also section 2.5.4, page 76). The ssDNA is $\sim 29 \mathrm{~nm}$ long and attached to an $11.7 \mathrm{~nm}$ long dsDNA strand, which in turn is bound to a surface. It is known from literature that dsDNA has a persistence length of around $50 \mathrm{~nm}[132,133]$, while the persistence length of ssDNA is around $2 \mathrm{~nm}$ [134-136]. It is thus a reasonable idealization to treat the dsDNA strand as straight and fixed compared to the ssDNA. A model sketch of the system under investigation can be found in figure 5.19 .

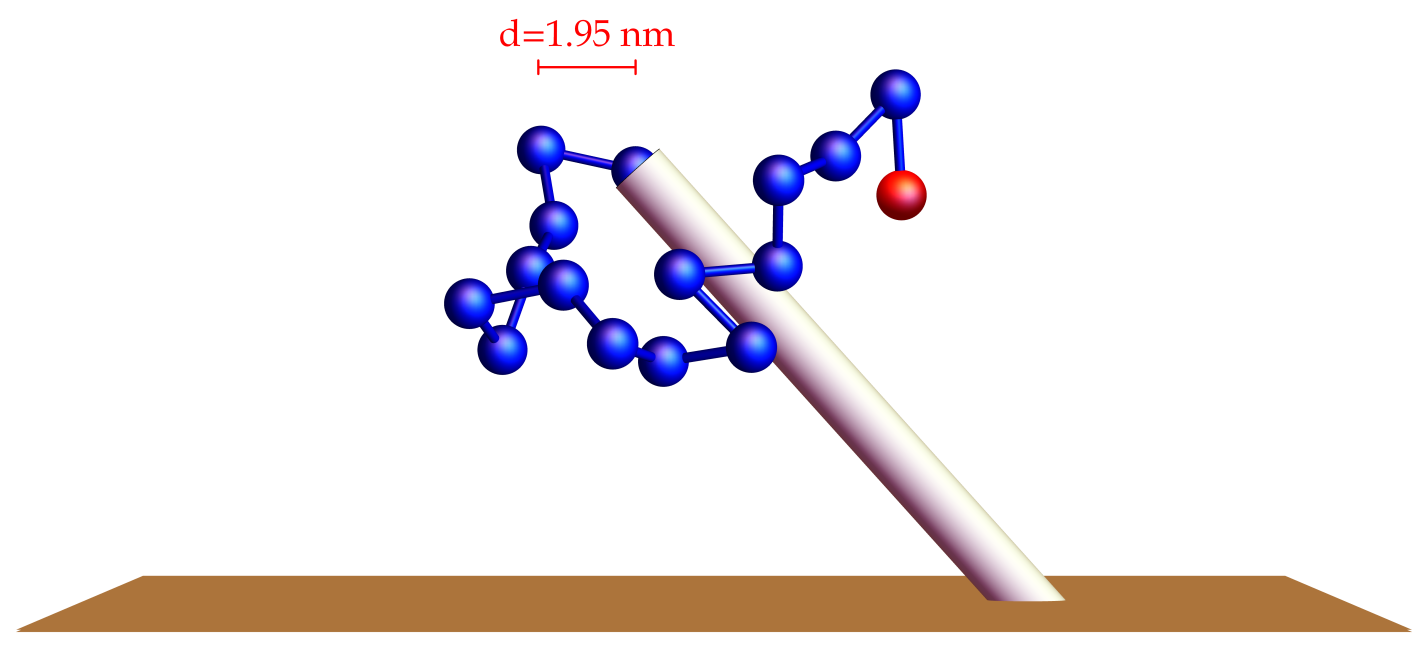

Figure 5.19: Sketch of the model system. Each bead represents three nucleotides. The bond length between them is fixed to $1.95 \mathrm{~nm}$. The chain's end is held fixed at the end of a stalk representing the dsDNA. The stalk is not part of the simulation, and is shown here only for visual purposes. The blue spheres cannot overlap with each other or the surface.

The ssDNA undergoes Brownian motion and its end is labeled with the fluorophore Atto647N. The observable of interest in the ssDNA's dynamics is the distance of its chain end (z-position) from the surface, because the fluorophore's intensity depends on this time-dependent distance. The z-position has an almost linear relationship with the experimentally measured intensity (see figure 2.14, page 78) whose autocorrelation function is being measured via dynaMIET. A typical experimentally measured FCS-curve is shown in figure 5.20. 


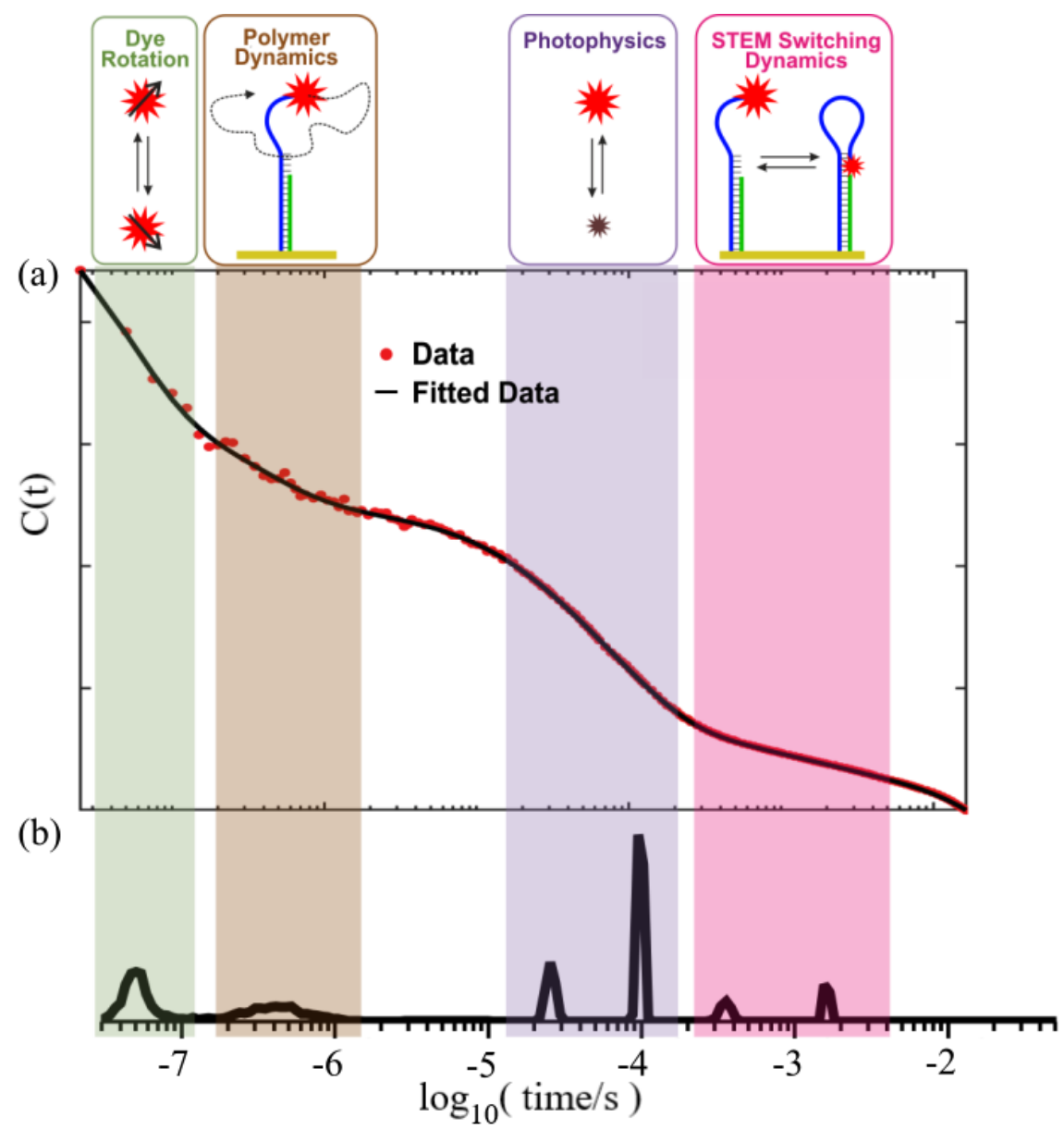

Figure 5.20: Experimental dynaMIET intensity-based correlation curve. The time span of six orders of magnitudes allows us to identify multiple dynamic processes which occur in the sample. (a) Exemplary fluorescence intensity autocorrelation curve obtained from dynaMIET surface scanning-based experiments at a salt concentration of $300 \mathrm{mM}$. (b) Characteristic time constants obtained from fitting the correlation curve with a sum of exponential functions. Shown are the amplitudes of the respective exponential function as a function of the time scale on which they decay. The top panel shows our preliminary identification of dynamic processes occurring on different time scales. The background colors were used to guide the eye. This figure was kindly provided to me by Roman Tsukanov.

The polymer's dynamics is not the only dynamic process influencing this curve - for example the photophysics of the fluorophore and its rotational dynamics must also do. The purpose of modeling dynaMIET experiments is to give an estimate for the time scale on which these intensity fluctuations occur in order to help interpret and verify experimental results. In particular, as a first step, the simulation results are supposed to test the hypothesis that the peak at a few hundred nanoseconds is due to polymer dynamics. In other words: The model's task is to deliver the order of magnitude on which the autocorrelation function of the stochastic intensity signal decays (reconfiguration time). At this stage, great accuracy of this time scale is not required. 
Method As before, we model the studied polymer as an inextensible, semiflexible bead-rod chain with hydrodynamic interactions and excluded volume effects. The novelty here is that the bound-end constraint applies. One chain end is bound to a point at a distance of $h_{0}=L \cdot \cos \phi$ away from the surface, where $L=11.7 \mathrm{~nm}$ is the length of the dsDNA strand and $\phi$ is the average tilt angle of the dsDNA strand (when $\phi=0$ the strand is parallel to the surface's normal vector). The angle $\Phi$ was experimentally determined via lifetime measurements to be $\sim 42$ degrees such that $h_{0} \approx 8.7 \mathrm{~nm}$. The equations of motion for the $N$ beads' position vectors $\vec{r}_{i}$ read

$$
\begin{aligned}
\mathrm{d} \vec{r}_{i}= & \beta \sum_{j=1}^{N} \mathbf{D}_{i j}^{\text {wall }}\left(-\vec{\nabla}_{j}\left(E-T S_{C}\right)+\lambda_{j} \vec{t}_{j}-\lambda_{j-1} \vec{t}_{j-1}\right) \mathrm{d} t \\
& +\left(\vec{\nabla} \cdot \mathbf{D}^{\text {wall }}\right)_{i} \mathrm{dt}+\sqrt{2} \sum_{j=1}^{N} \mathbf{B}_{i j} \mathrm{~d} \vec{W}_{j}
\end{aligned}
$$

where $\lambda_{N}=0$, and are supplemented by the constraints

$$
\text { and } \begin{aligned}
\vec{t}_{j} \cdot\left(\mathrm{d} \vec{r}_{j+1}-\mathrm{d} \vec{r}_{j}\right) \stackrel{!}{=} 0 \\
\vec{t}_{0} \cdot \mathrm{d} \vec{r}_{1} \stackrel{!}{=} 0
\end{aligned}
$$

where $j=1, \ldots, N-1$. Note that compared to the case of freely swimming chains in the previous sections, the additional bound-end constraint alters the entropic force $\vec{\nabla} S_{C}$. Moreover, we take the presence of the surface into account by using the Rotne-Prager-Blake diffusion matrix $\mathbf{D}^{\text {wall }}$ which (other than the diffusion matrix in free space) is not divergence free. Its divergence can be found in reference [96] (equation 14 therein). The bending energy $E$ includes the "zeroth" vertex placed at the origin (end of dsDNA stalk), which implies that we impose a favored initial direction to the ssDNA chain at its base where it is bound to the dsDNA stalk. The dsDNA stalk itself was not included in the simulations - its presence is only reflected by the bound-end constraint at the proper distance $h_{0}$ from the surface and by the first term in the bending energy. Representing the ssDNA by one bead per nucleotide $(N=45)$ has turned out to be numerically too expensive and thus we coarse grained three nucleotides into a single bead. This choice is arbitrary and was made simply because 45 is divisible by 3 . We will see below that this choice still allows us to reproduce experimentally found radii of gyration of ssDNA chains, while representing them with too few beads would not. Following this model, our ssDNA chain with 45 nucleotides translates into a chain length of 15 beads. Small-angle x-ray scattering (SAXS) experiments [134] report a bond length between two nucleotides of $6.5 \AA$, so we take the bond length between two of our beads to be $19.5 \AA$, resulting in a fixed contour length of $29.25 \mathrm{~nm}$. 
We further choose the excluded volume radius $R_{\mathrm{EV}}$ for each bead to equal its hydrodynamic radius $a$. This choice was made because of three reasons; (i) the diffusion matrix does not allow the beads to hydrodynamically overlap with the wall ${ }^{1}$, and rejecting time steps resulting in such overlaps prevents this from happening, (ii) the previously made choice of $R_{\mathrm{EV}}=d \cdot \sqrt{2} / 4$ is potentially too large due to the coarse grained value of $d=1.95 \mathrm{~nm}$, and (iii) it turns out that the model parameter $a \approx 0.25 \cdot d$ which we find later, while not preventing all crossing events, strongly prohibits them. Thus, the physics that excluded volume effects are supposed to add to the model are essentially captured.

The model includes two free parameters - the chain's bending stiffness $\sigma$ and the single-bead radius $a$ - whose values we need to estimate in order to run simulations. They were chosen such that (i) the model's radius of gyration

$$
R_{G}=\frac{1}{2 N^{2}}\left\langle\sum_{i, j=1}^{N}\left(\vec{r}_{i}-\vec{r}_{j}\right)^{2}\right\rangle
$$

equals the experimentally found value from SAXS measurements available in literature [134] and (ii) its translational diffusion coefficient $D$ equals that which we measured independently for the ssDNA strand using 2fFCS. The model's radius of gyration and diffusion coefficient are both calculated using MC sampling. Matching the two criteria for the two parameters was achieved as follows: The parameter $a$ influences $R_{G}$ only because $a=R_{\mathrm{EV}}$, but this influence is weak. Thus, an initial guess for $a$ was made, and $\sigma$ was tuned to match the experimental $R_{G}$ value. Next, $a$ was tuned while holding $\sigma$ fixed to match the experimental diffusion coefficient $D$. This change of $a$ then slightly altered the model's $R_{G}$ value, such that $\sigma$ needed to be slightly readjusted in turn. After a few iterations of this process, the model virtually precisely delivered the desired values for $R_{G}$ and $D$ simultaneously. All model parameters were thus determined and Langevin simulations can be run. For each tested parameter set, 40 independent trajectories were run. Each trajectory was at least $10^{4} t_{0}$ long, which is on the order of $100 \mu \mathrm{s}$ - roughly three orders of magnitude longer than the intensity autocorrelation time (see table 5.9). During each simulation, the chain end's distance from the surface $h(t)$ was kept track of as a function of time. Afterwards, it was translated into the intensity function $I(h(t))$ using the function shown in figure 2.14 (page 78). Finally, the autocorrelation function was calculated as

$$
g(\tau)=\frac{\langle\Delta I(t) \cdot \Delta I(t+\tau)\rangle}{\sigma_{I}^{2}}
$$

where $\Delta I=I-\langle I\rangle, \sigma_{I}^{2}=\left\langle\Delta I^{2}\right\rangle$ and the average $\langle\cdot\rangle$ was taken over all times and simulations.

\footnotetext{
${ }^{1}$ If they do overlap with the wall, the diffusion matrix $\mathbf{D}^{\text {wall }}$ is no longer positive definite such that the Cholesky decomposition $\mathbf{B}^{\text {wall }}$ does not exist.
} 
Diffusion coefficients The diffusion coefficient was measured for different salt concentrations in water. For salt concentrations of $150,300,500$ and $700 \mathrm{mM}$, we obtained values 86.5, 83.9, 82.7 and $82.9 \mu \mathrm{m}^{2} / \mathrm{s}$, the average value of which is $\hat{D} \approx 84.0 \mu \mathrm{m}^{2} / \mathrm{s}$.

Radius of gyration We have not measured the radius of gyration of the ssDNA chains ourselves, but utilize literature values from SAXS measurements [134], where the radii of gyration of poly-A chains ${ }^{2}$ of different chain lengths were investigated for different salt concentrations. Since the exact salt concentrations and chain length we are using had not been investigated in said study (see supplemental material therein), we interpolated their values in two steps. Firstly, we fitted $R_{G}$ as a function of chain length for each salt concentration independently, and inserted our chain length (45 nucleotides) into the fitted power law. For salt concentrations 25, 225, 525 and $1025 \mathrm{mM}$ this yielded $R_{G}$ values $3.96,3.81,3.71$ and $3.56 \mathrm{~nm}$ - the black dots in figure 5.21. Secondly, we heuristically fitted a monoexponential function with an offset to the so-found data points ( $R_{G}$ of 45 nucleotides as a function of salt concentration) and read off $R_{G}$ values for our salt concentrations (see above). We obtained values 3.87, 3.79, 3.71 and $3.64 \mathrm{~nm}$, the average value of which is $\hat{R}_{G} \approx 3.75 \mathrm{~nm}$.

Simulation results Since the experimental values for both $D$ and $R_{G}$ show a relatively weak salt dependence for the considered salt concentrations, we used the average values of $\hat{D} \approx 84.0 \mu \mathrm{m}^{2} / \mathrm{s}$ and $\hat{R}_{G} \approx 37.5 \AA$. The corresponding model parameters $a$ and $\sigma$ then represent the ssDNA for all salt concentrations at once. With the parameter values $N=15, d=1.95 \mathrm{~nm}$, $a=R_{\mathrm{EV}}=0.25 d$ and $\sigma=0.071 d$, the model chain possesses the same diffusion coefficient $\hat{D}$, radius of gyration $\hat{R}_{G}$ and contour length $L$ as the average experimental ssDNA chain. $1 \mu$ s of a simulated trajectory $h(t)$ and $I(t)$ is shown in figure 5.22. The resulting simulated intensity autocorrelation was sampled at the same times as in the experimental setting (the time bins in the FCS histogram) and was then fitted with a mono-exponential and with a double-exponential function (see figure 5.23).

In the experimentally accessible time regime ( $>25 \mathrm{~ns}$ ), the mono- and double exponential fits virtually coincide - their time constant (reconfiguration time) is $\tau_{1}=166 \pm 2 \mathrm{~ns}$. In the short-time regime ( $<25 \mathrm{~ns}$ ), the double-exponential fit additionally reveals a fast component of the polymer dynamics on a time scale $\tau_{2} \approx 12 \mathrm{~ns}$, which has thus essentially already decayed before the first experimental data point at $25 \mathrm{~ns}$. The amplitude of this fast component is around one order of magnitude smaller than that of the slower component.

\footnotetext{
2"A" stands for the nucleobase adenine. Our ssDNA chains also predominantly contain adenine.
} 


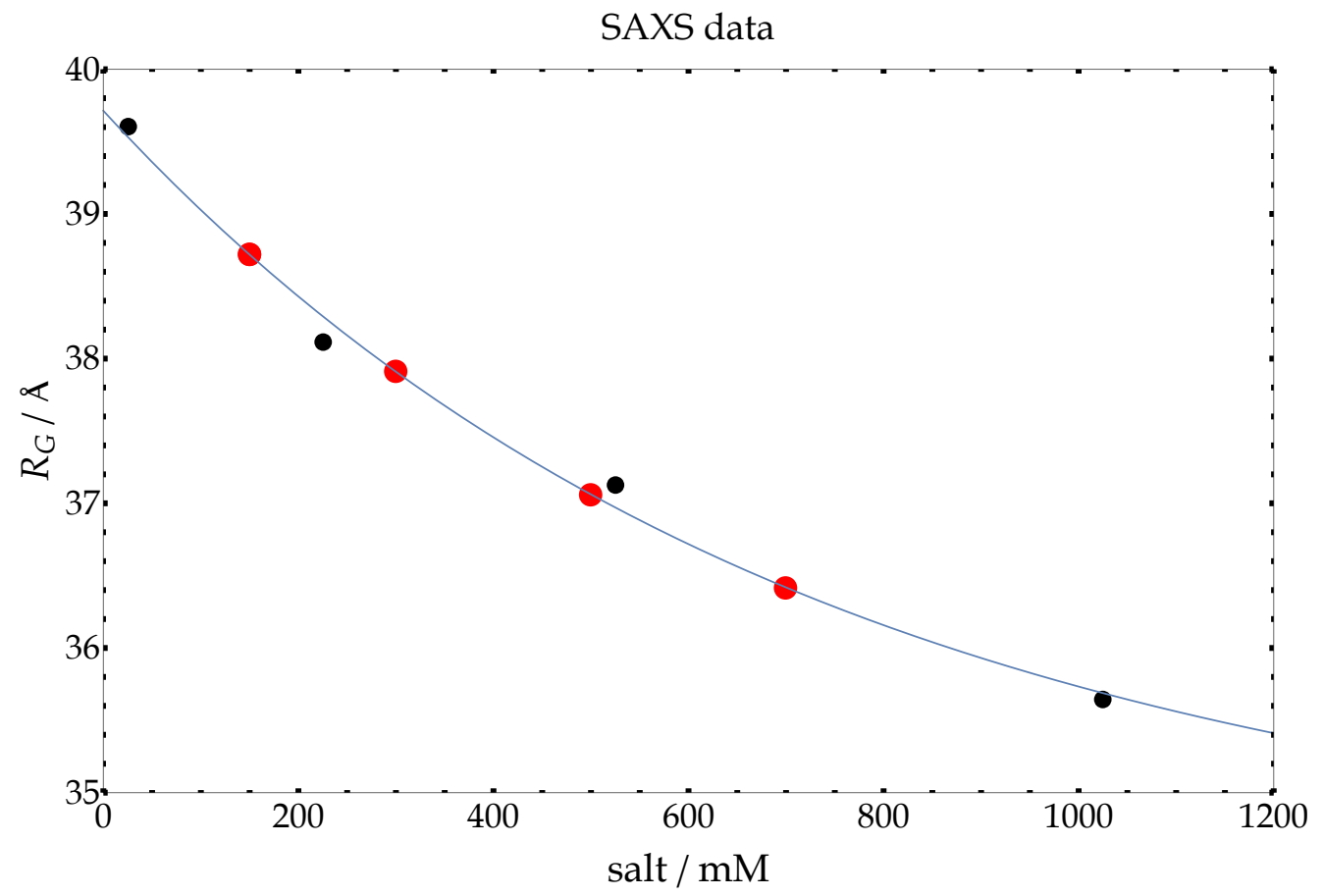

Figure 5.21: Interpolated $R_{G}$ values for different salt concentrations from SAXS measurement taken from reference [134]. Black dots show values interpolated to a chain length of 45 nucleotides (poly-A strands). Red dots show values which are further interpolated to the required salt concentrations using a heuristic monoexponential fit with an offset (blue line).

In order to further test the model parameters' influence on $\tau_{1}$, we ran two additional sets of simulations: The chain's stiffness was increased (decreased) while the bead size was decreased (increased) in such a way that the model's diffusion coefficient $D$ remained unchanged but $R_{G}$ did not. That is because the $R_{G}$ values were taken from literature studies for different chain lengths and salt concentrations as described above, whereas we measured the diffusion coefficients for our exact molecules ourselves. The bending stiffnesses in these additional simulations were chosen to be $\sigma=0$ and $\sigma=0.5 d$, and the corresponding bead sizes were $a=0.27 d$ and $a=0.21 d$ respectively. We remark that the radius of gyration cannot be reduced further down than $\sim 35.5 \AA$ in our model ( $\sigma=0$ ) as long as $N=15$. Lowering it further would require finer discretization of the chain, but our discretization turned out to be sufficient because $\hat{R}_{G}>35.5 \AA$. For all tested $R_{G}$ values, the corresponding persistence lengths $l_{P}$ and time constants can be found in table 5.9.

The autocorrelation time does depend on the radius of gyration, but it does not change its order of magnitude even when the radius of gyration is increased drastically (by more than $20 \%$ ). 


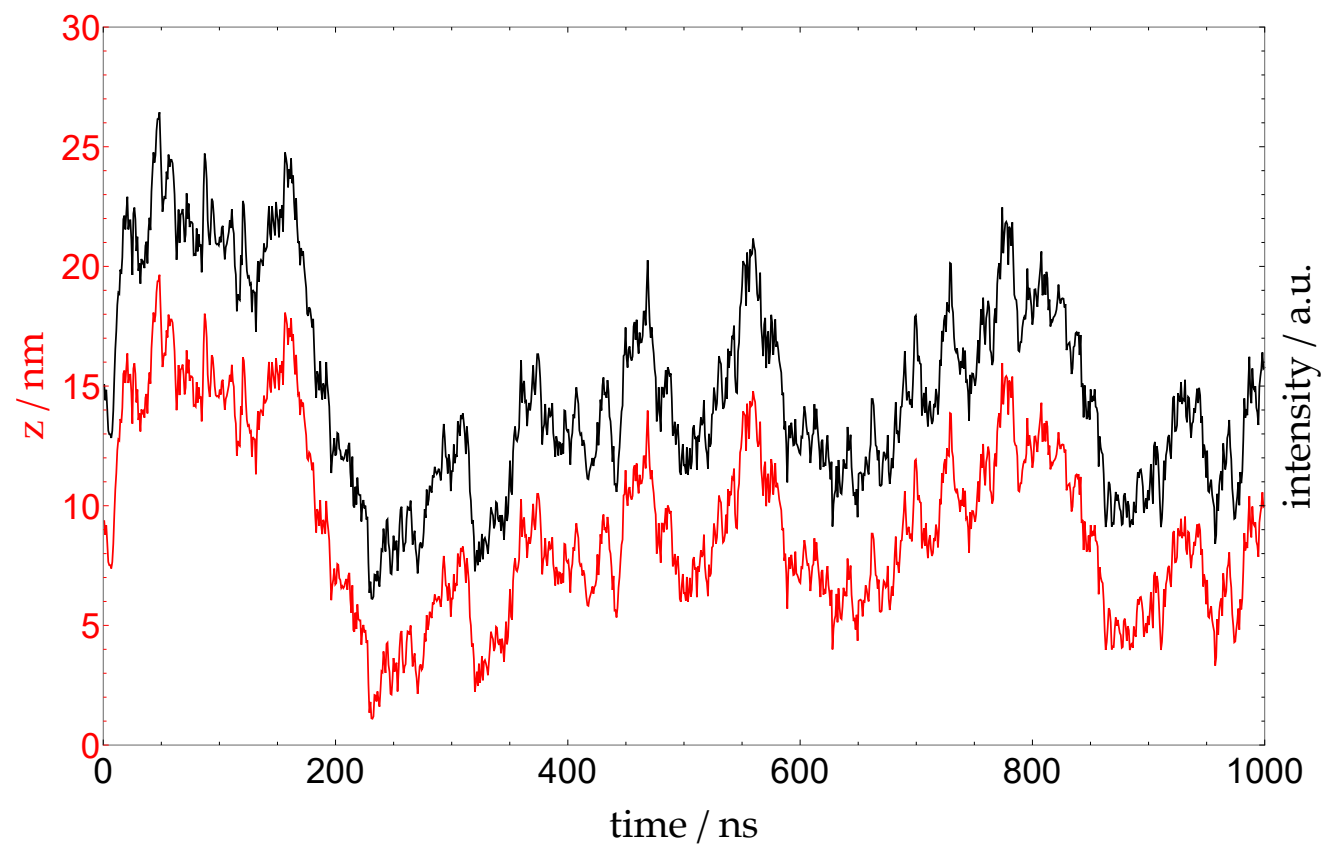

Figure 5.22: $1 \mu \mathrm{s}$ of a simulated trajectory. In red, we show the chain end's distance from the surface, and in black we show the corresponding intensity in arbitrary units. The two signals look almost identical because the relationship between height and intensity is virtually linear in this regime (see figure 2.14, page 78).

\begin{tabular}{ccc}
\hline \multicolumn{3}{c}{$D=84.0 \mu \mathrm{m}^{2} / \mathrm{s}$} \\
\hline$R_{G} / \AA$ & $l_{P} / \AA$ & $\tau_{1} / \mathrm{ns}$ \\
\hline 35.5 & 14.4 & $166 \pm 5$ \\
37.5 & 16.3 & $166 \pm 2$ \\
45.7 & 25.8 & $250 \pm 6$ \\
\hline
\end{tabular}

Table 5.9: The simulated parameter sets and corresponding mono-exponential time scales of intensity autocorrelation decay.

Conclusion The simulation results yield two useful pieces of information. Firstly, the experimentally measured autocorrelation of the polymer dynamics can be expected to decay on a time scale in the order of tens or hundreds of nanoseconds. Secondly, fitting it with a mono-exponential function is reasonable. As a side note, if a continuous excitation laser is used such that the fast polymer dynamics ( $<25 \mathrm{~ns}$ ) can be investigated, additional fast components of the polymer dynamics may be revealed. 


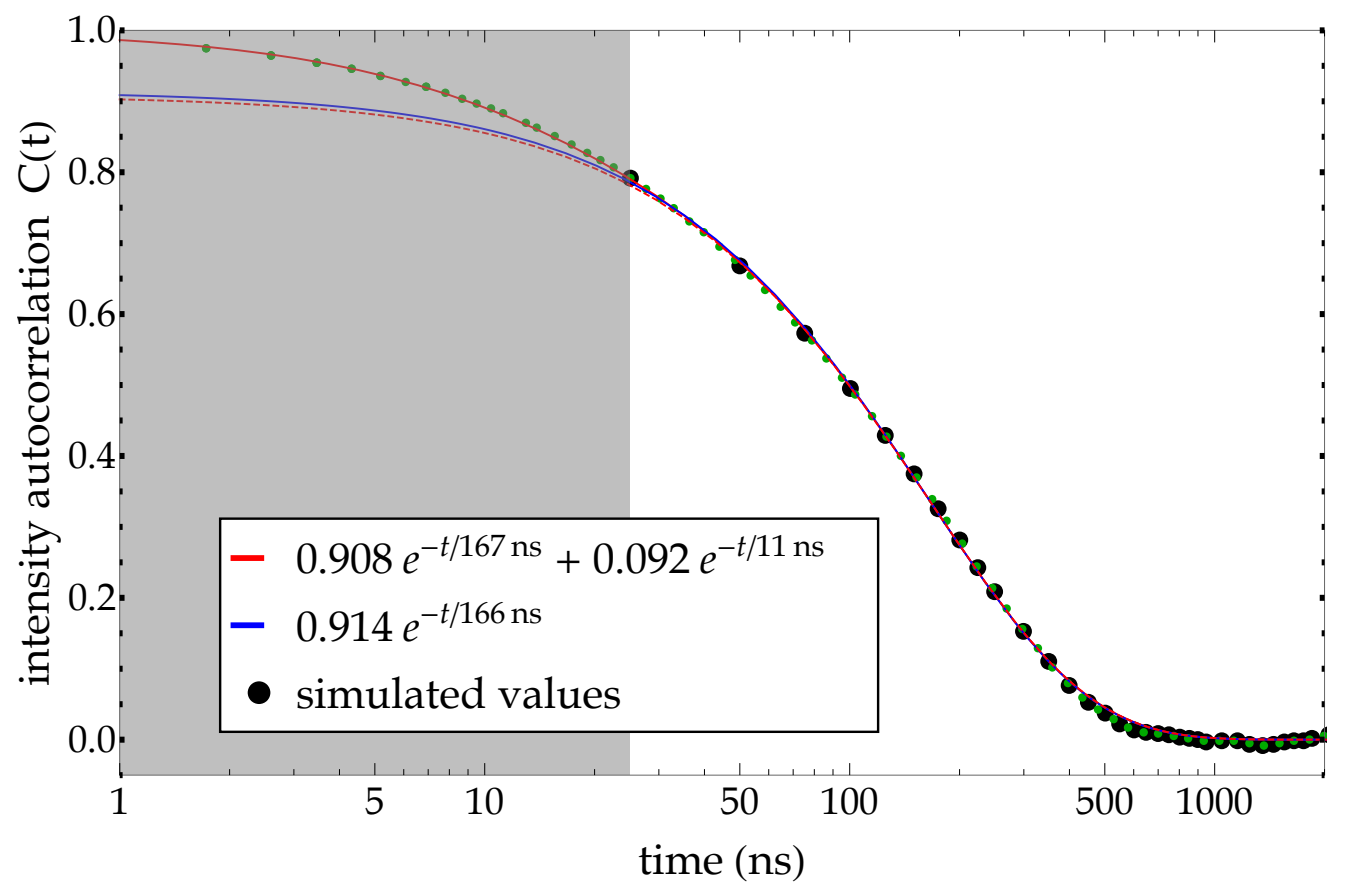

Figure 5.23: The autocorrelation $C(t)$ of the simulated intensity $I$, sampled at the same times as in experiments (black dots) and for interest's sake also sampled at logarithmically spaced times (green dots). The time axis is logarithmic. The grey area indicates the experimentally inaccessible time interval. The red solid line shows a double exponential fit to the black data points, and the solid blue line shows a mono-exponential fit to the black data points. The red, dashed line is the slower component (first term) of the double-exponential fit. As can be seen, it almost coincides with the blue solid line, meaning that the monoexponential fit is a good approximation to the data points in the experimentally accessible regime. 


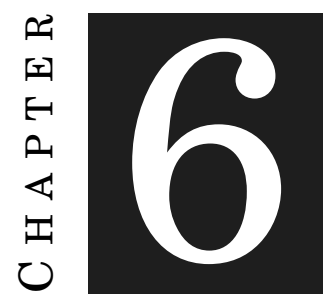

\section{DisCUSSION AND CONCLUSION}

$\mathrm{T}$ he results of this thesis will now be discussed. The findings for GS-repeats constitute the main experimental application of the polymer model we developed. Therefore, they enjoy the highest priority in this chapter, being followed up with the findings concerning the FG-proteins. The dynaMIET project is still unfinished as final experimental results are yet unavailable. Its state is briefly mentioned by the end of this chapter and further discussed in the next one. Our polymer model itself is reviewed by the end of this chapter (conclusion). Its behaviour was already discussed in section 5.1 (page 107), which will not be repeated here.

Contact rates The values of contact formation rates $k_{+}$are highly dependent on the photophysical details of contact formation. In our simulations, all these details are subsumed by the effective radius for instantaneous contact formation, for which we had chosen $R_{C}=5.4 \AA$ [117]. The appropriate numerical value for $R_{C}$, as well as the number of fluorophore-quencher collisions required for successfully forming a non-fluorescent fluorophore-quencher complex have been investigated using molecular dynamics simulations and experimental techniques [13, 117, 120], which share the fact that the investigated systems do not consist of energetically non-interacting spheres as is the case in our model. Some systematic bias of the modeled absolute values of fluorophore-quencher formation rates is therefore unavoidable for any beads model. The fact that our simulated rates are nonetheless within $\sim 17 \%$ of the experimental values is reassuring and confirms that the experimentally measured contact rates and hydrodynamic radius values are globally consistent with each other and our model. We reemphasize that the model parameters were not at all fitted to match the experimental rates' order of magnitude - we got this match for free. It can furthermore be observed that the magnitude of the simulated contact rates does not 
differ significantly between the candidate parameter sets. That is because these parameter sets attribute identical hydrodynamic radii to the chains. This is achieved by balancing the increase in hydrodynamic radius $R_{H}$ due to higher chain stiffness $\sigma$ with making the bead size $a$ smaller. The same balancing effect simultaneously takes place at the level of the contact rates' values: chain stiffness decreases the rates and making the beads smaller counteracts this decrease. Fitting the experimental rates' exact values is therefore in some sense redundant with fitting the experimental hydrodynamic radii. This circumstance would make a two-dimensional parameter search based on these two criteria difficult. Instead, our method of fitting the dimensionless power law exponent and tail effect seems sensible. If, however, the precise values of simulated contact rates become of interest in the future - for example to compare them for different amino acid sequences - I suggest using the critical contact radius $R_{C}=5 \AA$, which we had estimated to give the correct contact rate values for the simulated GS-repeats. For these proteins, the model has performed exceptionally well, which is why they are well-suited to serve as a calibration.

Previous literature on contact rates Several experimental studies measured the rate with which two sites on a protein come together [3-13]. The loop formation rate in disordered proteins was found to have a power law dependence on their chain length [3, 6, 7], as long as the chains are no shorter than about 10 amino acids [14]. The order of magnitude of our experimentally measured contact rates $\left(\sim 10^{7} \mathrm{~s}^{-1}\right)$ is in agreement with that measured previously for GS-repeats. Reference [6] finds them to be on the order of $10^{7}-10^{8} \mathrm{~s}^{-1}$ for proteins with 10 or fewer amino acids (1999). Reference [14] confirms this and observes the rates dropping to $\sim 5 \cdot 10^{6} \mathrm{~s}^{-1}$ for chain lengths up to 60 amino acids (2003). Reference [3] reports slightly larger values, although still on the same order (2007). These three studies did not use PET-FCS as we did, but an outdated method called triplet-triplet energy transfer (TTET), in which fluorescence quenching is not instantaneous. It occurs with a rate similar to $k_{+}$whenever fluorophore and quencher are in contact, thus requiring an additional fit parameter when interpreting FCS-curves. A more recent study [13] from 2010, which uses PET-FCS for GS-repeats as we did, considers chain lengths up to 15 amino acids and also finds contact rates on the order of $10^{7} \mathrm{~s}^{-1}$. On the theoretical side, several studies [19-25] delivered explicit but approximate expressions for contact rates which can be evaluated once a polymer model has been chosen. The most commonly used theory among these is the Szabo-Schulten-Schulten (SSS) theory [19]. In a nutshell, SSS theory assumes the end-to-end dynamics in a polymer chain to be a one-dimensional Markov-process. Given the end-to-end probability distribution $p(R)$, one immediately finds the associated free energy function $F(R)$ by inverting the Boltzmann distribution. Upon insertion of $F(r)$ into the one-dimensional FokkerPlanck equation, SSS theory yields an explicit expression for the contact rate. For Gaussian end-to-end statistics, this expression (integral) can be solved analytically [10] and reads

$$
k_{+} \propto\left\langle R^{2}\right\rangle^{-1.5} \propto L^{-1.5 v}
$$


which is a power law, since typically $\left\langle R^{2}\right\rangle \propto L^{v}$. Studies which numerically evaluate theory-based expressions for $k_{+}$typically confirm its power law behavior as a function of chain length. Different scaling regimes (depending on critical contact radius, chain length and persistence length) with different power law exponents exist [26-35], however, and deviations for too short, semiflexible chains are observed [40,41]. Purely computational studies for coarse-grained polymers (less realistic than ours though) confirm this power law behavior [36-39] for chains significantly longer than their persistence length. Considering how accurately our own simulated contact rates follow power laws (see figure 5.11, page 122), we can conclude that the true relation between contact rates and chain length - if it is not one exactly - is at least well-approximated by a power law.

Power law exponent Our results show that the power law exponent $\epsilon$ depends (i) only weakly on hydrodynamic bead size $a$, (ii) but strongly on bending stiffness $\sigma$. Without hydrodynamic interactions, the first statement is trivial: the bead size only linearly re-scales the time scale of the dynamics for all chain lengths in the same manner and will hence not change the power law dependence on chain length. With hydrodynamic interactions, our results show that while the contact rates depend non-linearly on $a$, they do so in a similar fashion for all chain lengths. The second statement, that the power law behavior strongly depends on bending stiffness $\sigma$, can be understood as follows. For the dynamics of less stiff chains, excluded volume effects become more important as beads collide statistically more often. This alters the chain's end-to-end distance (R) scaling behavior with chain length $L$; writing $\left\langle R^{2}\right\rangle \propto L^{v}$, one has $v=1$ for an ideal chain and $v>1$ for self-avoiding random walks (Flory chains, see page 65). This scaling behavior can be linked to the contact rate's scaling behavior in light of the SSS theory assuming Gaussian end-to-end statistics (see equation 6.1 above). Therefore, the power law exponent $\epsilon$ is altered from -1.5 for ideal chains towards lower values for self-avoiding chains. With a simulated exponent of $-1.52 \pm 0.01$, our simulations without excluded volume effects (see table 5.5, page 126) confirm this interpretation excellently. Although SSS-theory is oversimplified as it assumes the end-to-end dynamics to be Markovian, its predicted relationship between contact-rate scaling and end-to-end distance scaling seems correct. A similar impact of excluded volume effects on the dependence of the power law exponent on persistence length has also been found in reference [7]. Note that the quantitative relation between power law exponent and chain stiffness can be expected to depend on the model's excluded volume radius for each monomer. We emphasize again that the value we used ( $\sqrt{2} / 4$ times a bond length) is the minimum value preventing bonds from passing through each other and was chosen only for this geometric reason. We remark that, since excluded volume effects lower the contact rates, a smaller power law exponent (steeper slope in log-log-plot) implies that excluded volume effects play a more important role in longer chains.

The exponent value of $-1.86 \pm 0.15$ that we found is relatively small compared to findings of other studies of the same types of proteins (GS-repeats). Reference [6] reports a value of $-1.36 \pm 0.26$ for 
very short chains (4-10 amino acids only) where deviations from sufficiently long polymer chains (i.e. significantly longer than their persistence length) may be expected. Reference [13] reports a value of $-1.4 \pm 0.2$ using chain lengths of 10 to 30 amino acids. Reference [14] used chains even longer than ours (up to 56 amino acid residues) and found a value of $-1.72 \pm 0.08$, which is within our error margin. The last reference determined also the exponent for serine-only proteins (measuring no chains longer than 12 amino acids though) and found a value of $-2.1 \pm 0.3$.

When fitting a power law via least squares, we weighted each rate according to its error (weight $\propto$ 1/error ${ }^{2}$ ) which we took, for the experimental data, to be proportional to the rate's value itself, and, for the model, to be proportional to the statistical error over the ensemble of simulated trajectories. We fitted the power law to the contact rates as a function of contour length, including the fluorophore, whereas the references above fitted them as a function of the number of amino acids. We have tested that neglecting the fluorophore's contribution to the chain's contour length leads to a larger (smaller in magnitudes) fitted exponent for our data $(-1.68 \pm 0.15)$, which thus seems a likely source of discrepancy with other studies.

Tail effect We found that the tail effect depends predominantly on the hydrodynamic bead radius $a$, but not on the chain stiffness $\sigma$. We interpret this result as a clear indicator that the tail effect originates from the hydrodynamic drag that the extra beads behind the quencher add to its movement (see also reference [11]). Larger beads (i) diffuse more slowly as individual particles, but (ii) are hydrodynamically more strongly coupled and fluctuate more cooperatively. The second effect is dominated by the first when considering e.g. the absolute value of contact rates or the translational diffusion of the entire chain. The tail effect value, however, is a dimensionless ratio of rates which remains unaffected by the first effect. Therefore, the more cooperative motion of quencher and tail reduces the tail effect when the hydrodynamic bead size $a$ is increased. One may expect that the tail effect would also depend on persistence length as the tail is, on average, farther away from the quencher for stiffer chains, which reduces the hydrodynamic coupling between them. Our simulations show that this effect plays a minor role when compared to the impact of bead size. Another effect that contributes to the tail effect is that the tail leads to an additional sterical hindrance during loop formation. Our simulations without excluded volume show that this effect exists, but is relatively weak compared to the impact of changing $a$, which confirms again that solvent friction is the main origin of the tail effect.

More detailed investigations of the tail effect than ours are available in literature. For instance, reference [5] experimentally found that the tail effect increases as a function of the tail's length, and as such converges towards a value where the contact rate is about 2.5 times lower than the rate with no tail. Reference [11] systematically studied the tail effect in single-stranded DNA and in simple coarse-grained simulations. They demonstrated that the dependencies of contact rates as a function of quencher position (for different contour lengths) can be mapped to a universal 
curve when properly normalized. Reference [137] analytically investigated the tail effect in the Rouse chain (Gaussian chain), though not at the level of contact rates, but of reconfiguration times (time scale of end-to-end distance fluctuations).

For our purposes, we reiterate that the tail effect is a purely dynamical effect which cannot be inferred from the chain's statistical properties (Boltzmann distribution). It is therefore a good test for a dynamic model to reproduce an experimentally measured tail effect because correct chain statistics alone do not suffice to pass this test.

Fit parameter values We determined the persistence length of GS-repeats to be $l_{P}=5.2 \pm$ $1.9 \AA$, and the hydrodynamic radius of one of their amino acids to be $a=3.5 \pm 0.7 \AA$. For comparison, other authors found hydrodynamic radius values of $4.1 \AA$ [138], $4.5 \AA$ [139], $5.1 \AA$ [140], $>3.6 \AA$ [141] and $5.3 \AA$ [142] for single amino acids. These values were found by comparing experimentally measured diffusion coefficients with those of bead models of proteins within the Rotne-Prager approximation. Our value is on the lower end of this spectrum, which is reasonable, taking into account that glycine and serine are comparatively small amino acids. It should be noted that all these values are larger than half the distance between adjacent $C_{\alpha}$ atoms, which is equal to $1.9 \AA$. Thus, hydrodynamically speaking, neighboring beads overlap. Citing reference [55], "this reflects the fact that: 1) the interior of the protein is densely packed, 2) the side chains of amino acids are usually longer than $1.9 \AA$, and 3) the protein is covered by the hydration layer of tightly bound water molecules."

Finally, our persistence length value is in good agreement with findings of other authors for intrinsically disordered proteins: $l_{P}=4 \pm 0.7 \AA$ [143] (measured from Förster resonance energy transfer experiments), $l_{P}=4 \AA$ [144] (fitting a worm-like chain model to end-to-end distances extracted from the protein data base), $l_{P}=6.4 \AA$ [10] and $l_{P}=4$ to $5.5 \AA$ [7] (both comparing experimental contact rates with SSS theory inserted with the end-to-end statistics from a worm-like chain, the latter study taking into account excluded volume effects).

The model's failure in reproducing FG results It turns out that applying the exact same model and fitting procedure, which worked splendidly for GS-repeats, to the internal-to-end contact rates and hydrodynamic radii measured for the FG-proteins was unsuccessful. Candidate parameter sets reproducing the experimental hydrodynamic radius values did not reproduce the correct contact rate values. The simulated contact rates of the FG-protein are a factor of 2 to 5 too large when using the same experimental flurophore-quencher pair (Atto655 with tryptophan) and critical contact radius (5.4 $\AA$ ) as used for the GS-repeats. More importantly, the simulated power law exponents (between -1.9 and -1.6 ) do not match the experimental one which is $-1.07 \pm 0.09$ (see figure 5.18, page 132). It is thus clear that none of the candidate parameter sets can be regarded as well-fitting. It would be possible to try out parameter sets with an even greater chain stiffness $\sigma$, but the exponents' trend already indicates this task's futility. 
We must hence discuss why model and experiment do not fit together for these proteins. There are three major differences to the GS-repeats. Firstly, the quencher tryptophan is not placed at the chain end but within the chain. This will likely make it more difficult for the quencher to diffusively come together with the fluorophore due to steric hindrances. The model accounts for this at least partly as it contains excluded volume interactions of the central backbone. It is possible, however, that the sterical hindrance is more crucial in the real molecules because the aromatic rings of tryptophan and Atto655 must properly stack together to form a nonfluorescent complex. The model treats them both as spheres with isotropic interaction, thus possibly overestimating the contact rate. Secondly, the FG-protein is highly heterogeneous, whereas the GS-repeats are homogeneous. In terms of our model, this may correspond to the bending elasticity $\sigma$ and the amino acids' hydrodynamic radii $a$ varying along the chain. The model does not take heterogeneities into account and can hence be expected to fit GS-repeats better than FG-proteins. GS-repeats are known to be disordered proteins, but we cannot be certain that the more complicated amino acid sequence of the FG-proteins does not result in remnants of secondary structures. Thirdly, the model does not include internal friction. Internal friction is a dissipative mechanism which is known to play a role in the dynamics of proteins in general $[145,146]$. Like solvent friction, internal friction is caused by interactions between the degrees of freedom whose dynamics is explicitly considered (the protein's backbone) and faster variables whose is not. For solvent friction, these interactions are collisions with water molecules, which statistically decrease the relative velocity between backbone and fluid. Internal friction is less well understood, but believed to be an analogous interaction of the chain with itself, slowing down conformational dynamics: When a bending angle changes, the amino acid's side chains must move along, but they are not explicitly considered at our level of description, and so they statistically add a friction force dampening the relative motion of neighboring parts of the protein. In a sense, both friction mechanisms can thus be considered to be coarse-graining artifacts. Our model does not include internal friction for the following reasons: (i) it requires an additional free parameter, and we tried to keep the number of parameters as small as possible; (ii) experiments have shown that the contact rates in GS-repeats exhibit an inversely linear viscosity dependence [3] which indicates that solvent friction and not internal friction is the dominant dissipation mechanism for GS-repeats; and (iii) it cannot be implemented in a trivial, straightforward manner. In any case, internal friction slows down conformational dynamics, which would be an appropriate correction for our model to better match the FG-proteins: the simulated contact rates are too large compared to the experimental ones. Moreover, it is analytically known from Gaussian chains [70] that the influence of internal friction (compared to solvent friction) decreases with chain length. This implies that including internal friction in our model would lead to an increased power law exponent of internal-to-end contact rate versus quencher position (flatter slope in log-log plot because points on the left are decreased further). This would further improve our model's fit quality for FG-proteins. 
Moreover, internal friction only influences the conformational dynamics of the simulated chain, and not its translational diffusion as a whole, thus leaving the hydrodynamic radii and candidate parameter sets untouched. To conclude, the lack of internal friction hence seems to be a likely cause of the mismatch between the existing model and the experimental data for FG-proteins. In the next chapter we suggest possible ways to take internal friction into account in our model.

Systematic discrepancies between model and experiment Other than the obvious neglect of quantum and relativistic effects, chemical transformations and internal friction, there are four systematic differences between our model and real proteins which deserve mentioning. First is hydrodynamics - the continuum description of water becomes questionable as we approach length scales on the order of individual water molecules (a few Angstrom). We use the Rotne-Prager approximation to solve the Stokes equation, which is known to reproduce more accurate higher-order methods very well [55]. Particularly vicious thermal fluctuations may temporarily cause high thermal velocities for which inertial effects play a role, thus invalidating the Stokes equation itself. Estimating an object's Reynolds number $\mathrm{Re}=\rho r_{0} v_{0} \eta^{-1}$ in water requires knowledge of the object's typical size $r_{0}$ and its velocity $v_{0}$ relative to the fluid. Taking $r_{0}$ as the hydrodynamic radius of the GS-repeats and $v_{0}$ as their thermal velocity from the equipartition theorem ${ }^{1}$ for the kinetic energy $v_{0}=\sqrt{3 \cdot T / m}$, with $m$ being the molecule's mass [124, 147], yields $\mathrm{Re} \approx 0.05$, meaning that the neglect of inertial effects is justified, but not perfect. Secondly, the geometry of our model chain is that of a discrete elastic rod of fixed length (bead-rod chain), while proteins possess a finite stretching stiffness. The stretching stiffness in proteins is large enough for bond fluctuations to be very small and much faster than conformational dynamics, however [148]. More importantly, the geometrically allowed deformations in proteins are bond rotations around their $N-C_{\alpha}$ and $C_{\alpha}-C$ bonds (also known as the angles $\Phi$ and $\Psi$ ), and not free rotations of each tangent vector on a spherical shell as is the case in our model. For too short chains this will impact the values the end-to-end vector can take on, but for a sufficient number of degrees of freedom, such atomistic details lose their relevance (which is the whole point of statistical mechanics). Thirdly, we have not discriminated between amino acids, whether they are glycine, serine or tryptophan. Tryptophan, which is by far the largest of the three, may slow down the end-to-end dynamics more than glycine or serine would in its stead, especially when placed at the chain's end. A more detailed description may take this into account by employing different bead sizes, which would come at the cost of introducing new parameters to the model. Fourthly, molecular dynamics simulations have shown [13] that the formation of intrachain hydrogen bonds influences the end-to-end dynamics, an effect that our model does not capture since we included only steric interactions between non-neighboring amino acids.

\footnotetext{
${ }^{1}$ It reads $\frac{m}{2}\left\langle v_{0}^{2}\right\rangle=\frac{3}{2} T$.
} 
Conclusion In this thesis, we have combined the existing theories of overdamped hydrodynamics, discrete differential geometry and constrained Brownian motion into a polymer model. Our model is fairly realistic as it includes solvent friction, hydrodynamic interactions, bending rigidity, excluded volume effects and also takes into account the attached fluorophore. This realism came at the cost of the model losing analytical solvability and being treatable only computationally. Generally speaking, adding effects to a mathematical model in pursuit of making it more realistic entails introducing free parameters, which quickly become too numerous to be quantified. We have succeeded in utilizing all experimental information at our disposal in order to compose a realistic model, while keeping the number of free parameters to a minimum: The number of amino acids (chain length), the medium's temperature and viscosity, the fluorophore's properties and the distance between $C_{\alpha}$ atoms in proteins are all available to us. Our model is capable of taking all these pieces of information into account and consequently includes only two fit parameters. As a second step, we designed a fitting routine for estimating these two remaining parameters from systematically measured hydrodynamic radii and contact rate values of IDPs. The crucial "trick" was to further simplify the parameter search process in two dimensions: Making use of the fact that hydrodynamic radii can be calculated much faster than contact rates can be simulated, the parameter search was reduced to take place in a single dimension: Brownian dynamics simulations must only be run along the curve of candidate parameter sets.

We have therefore succeeded in developing an efficient computational method for interpreting single-molecule experiments, which explicitly delivers molecular properties of the proteins. For GS-repeats, considered an ideal disordered protein without internal friction or secondary structures, our model has performed excellently. It is simultaneously able to reproduce the hydrodynamic radius values for different chain lengths, the contact rate values for different chain lengths, the power law exponent and the tail effect. We found out that GS-repeats are well described by a generic semiflexible chain which represents the proteins by one bead with a hydrodynamic radius of $3.5 \pm 0.7 \AA$ per amino acid and has a persistence length of $5.2 \pm 1.9 \AA$, and that excluded volume effects are a crucial ingredient in the model. Thanks to the model, we also gained insights into the microscopic mechanisms which lead to the experimentally observed values of $R_{H}, k_{+}$, the power law exponent and the tail effect. At the same time, the model fulfills all available analytical benchmarks, and yields a scaling behavior of the contact rates which is explained by SSS theory. The fitting process has to take a large number of experimental results into account, but can be performed within a few days on a computational cluster such as the one I had access to. We can therefore conclude that for "ideal" molecules such as GS-repeats, we have solved the task we set out to tackle. However, the model has failed in matching experimental results for FG-proteins - an inhomogeneous amino acid sequence which may include secondary structures and/or internal friction. It remains to be seen just how ideal proteins need to be in 
order to be successfully investigated with the current method. We have further demonstrated that the model is not at all limited to being applied to a combination of PET-FCS and 2fFCS experiments - it also delivers quantitative and testable predictions for the outcome of dynaMIET experiments. Possible directions of future work are discussed in the next chapter.

Determining persistence lengths and hydrodynamic properties of proteins is not a novelty, and neither is studying scaling exponents in polymer theory. Our achievement here was that we have built a link between these topics and state-of-the-art single molecule experiments by combining them with modern hydrodynamics, statistical mechanics and differential geometry, thereby exploiting the full potential of theory, simulation and experiment working hand in hand. 



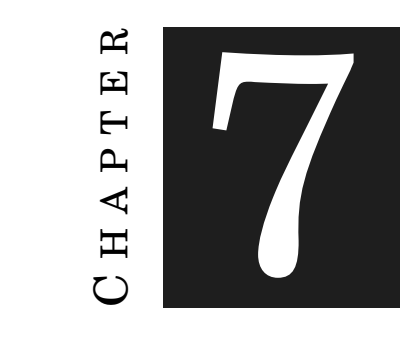

\section{OUTLOOK}

Follow up work to this thesis should go in five directions, which will now be discussed.

\subsection{Dynamics in generalized coordinates}

One of our model chains consisting of $N$ beads with $N-1$ inextensibility constraints effectively has $2 N+1$ degrees of freedom. Nonetheless, our inherently Cartesian description of the chain paired with introducing one Lagrangian multiplier per constraint results in $4 N-1$ variables whose values must be determined in each numerical time step. The routine for solving the constraints includes the inversion of an $N-1 \times N-1$ matrix, as does evaluating the entropic force $\vec{\nabla} S_{C}$. Furthermore, determining the diffusion matrix $\mathbf{D}$ requires the evaluation of $\propto N^{2}$ terms, after which its Cholesky decomposition must be calculated (time $\propto N \ln N$ ). Physically, the mean time between contact events grows as $N^{1.5-2.0}$. Taken together, these scaling laws severely limit the maximum chain length for which we can realistically simulate sufficiently long trajectories to around $\approx 60$. It is therefore desirable to describe the polymer dynamics in a lower-dimensional phase space. This can be achieved in two ways.

Firstly, the dynamics itself can be modeled in a simplified manner, e.g. in a one-dimensional picture where the only degree of freedom is the polymer's end-to-end distance. This path we should not take because it contradicts the purpose of our model - we want to learn about molecular properties and have at hand the full dynamics of the polymer, regardless of which observables are compared with experiments. For example, the tail effect cannot emerge from a one-dimensional picture - it would need to be encoded in the model by hand which undermines our philosophy of modeling: the model's behavior should be observed, not dictated. 
Secondly, lowering the effective number of degrees of freedom can be attempted within our polymer model without altering its physics. The idea should be completely analogous to the equivalence between the Lagrange I and Lagrange II formalism in classical mechanics: instead of imposing constraints on a Cartesian system by using Lagrangian multipliers, the dynamics may be described in terms of generalized coordinates which automatically satisfy the constraints. The set-out task would be to derive an equivalent Langevin equation not for the beads' Cartesian coordinates, but for a suitable parametrization of their tangent vectors (indicatrix). In practice, spherical coordinates $\left(\phi_{i}, \theta_{i}\right)$ could be used, although I suspect that the variables $\left(\phi_{i}, \cos \theta_{i}\right)$ yield a more practical description because they are uniformly distributed in absence of a bending energy and are "properly aligned" in its presence (see also pages 94 and 28). While in regular calculus transformations between variables are easily executed by using the chain rule, in stochastic calculus the rules for such transformations depend on whether the Itô or Stratonovich formalisms are used. Within either of them, some version of Itô's lemma [1] must be employed. For the Itô formalism we used in this thesis and a Langevin equation $\mathrm{d} x=a \mathrm{~d} t+b \mathrm{~d} W$, it states that the Langevin equation for a function $f(x)$ reads

$$
\mathrm{d} f=\left(a \partial_{x} f+\frac{1}{2} b^{2} \partial_{x}^{2} f\right) \mathrm{d} t+b \partial_{x} f \mathrm{~d} W
$$

which does not simply equal $\partial_{x} f \mathrm{~d} x$ as would be expected from regular calculus. Applying this lemma to our (Cartesian) Langevin equation carries over the terms including the Lagrangian multipliers as well as the entropic pseudoforce into the generalized coordinates' dynamics. Since, like the Cartesian description, the generalized description must be able to distinguish between a freely swimming chain and a chain with one end bound to a surface, it is impossible that the terms including constraining forces are simply "projected out" by the variable transformation. The information about one chain end being bound (or not) must be preserved upon translation to generalized coordinates. Another useful test for correctly preserved dynamics is the tail effect as explained before, it is a dynamical property which does not follow from correct conformational statistics. Moreover, the diffusion matrix is a function of the Cartesian coordinates, which may be tedious to evaluate in generalized coordinates, and the same holds true for the stochastic force term as its autocorrelation is given by the diffusion matrix.

It should be subject to future work to investigate whether or not these challenges can be overcome in such a way that numerical integration of our Langevin equation is made significantly more efficient by using generalized coordinates. To a reader pursuing this goal, I would like to point out that literature extending my knowledge on constrained Brownian motion (also in generalized coordinates) is available and may prove helpful [63, 65-67, 149-151]. 


\subsection{Investigating dynaMIET}

As soon as experimental results become available, a more thorough investigation of the polymer dynamics near a surface can be performed within our model. For instance, I have not yet studied the influence of the model parameters on the reconfiguration time and the shape of the autocorrelation function, or the scaling behavior with chain length, but in principle all this can already be achieved without altering the model. Like in PET-FCS, the molecules studied via dynaMIET can already be subjected to $2 \mathrm{fFCS}$ measurements, and the measured hydrodynamic radius be taken into account by the model. In the future, a combination of all three methods may be used in order to test the model's overall consistency, or to estimate the strength of internal friction, which influences the reconfiguration (dynaMIET) and the contact rate (PET-FCS), but not the hydrodynamic radius (2fFCS).

\subsection{Internal friction}

Generally speaking, internal friction is a relevant dissipative mechanism in the dynamics of IDPs $[145,146]$. It is currently not known, however, what exact microscopic mechanisms cause it, or how to implement it in a coarse-grained polymer model such as ours. A commonly used heuristic approach is that chosen in the Rouse-model with internal friction ("RIF") [70] whose Langevin equation reads

$$
\gamma \mathrm{d}_{t} \vec{r}_{n}=\left(\kappa+\zeta_{i} \mathrm{~d}_{t}\right)\left(\vec{r}_{n-1}-2 \vec{r}_{n}+\vec{r}_{n+1}\right)+\vec{\xi}
$$

The novel term here is the time-derivative on the right-hand side. It describes a force which is opposed to the relative velocity between the monomer $\vec{r}_{n}$ and its two neighbors. It is a linear term such that the Rouse model remains analytically solvable. The prefactor $\zeta_{i}$ tuning the strength of internal friction is analogous to the friction coefficient $\gamma$ doing so for solvent friction. Heuristically adapting this force term into our nonlinear, geometrically constrained Langevin equation leads to

$$
\begin{aligned}
\mathrm{d} \vec{r}_{i}= & T^{-1} \sum_{j=1}^{N} \mathbf{D}_{i j} \cdot\left[-\vec{\nabla}_{j}\left(E-T S_{C}\right)+\lambda_{i+1} \vec{t}_{i+1}-\lambda_{i} \vec{t}_{i}+\zeta_{i}\left(\mathrm{~d}_{t} \vec{r}_{j-1}-2 \mathrm{~d}_{t} \vec{r}_{j}+\mathrm{d}_{t} \vec{r}_{j+1}\right)\right] \mathrm{d} t \\
& +\sqrt{2} \sum_{j=1}^{N} \mathbf{B}_{i j} \cdot \mathrm{d} \vec{W}_{j}
\end{aligned}
$$

These updated equations of motion are not yet well-suited for numerical integration, because they are not explicitly solved for the increment $\mathrm{d} \vec{r}_{n}$. Fortunately, the increments enter the right-hand side in a linear manner, such that the Langevin equation (in $3 N$-dimensional vector notation) 
can be rearranged as follows:

$$
\begin{aligned}
\mathrm{d}_{t} \vec{r} & =\mathbf{D} \cdot\left(\vec{F}+\zeta_{i} \mathbf{M} \cdot \mathrm{d}_{t} \vec{r}\right) \\
\Leftrightarrow \quad\left(\mathbb{1}-\zeta_{i} \mathbf{D} \cdot \mathbf{M}\right) \cdot \mathrm{d}_{t} \vec{r} & =\mathbf{D} \cdot \vec{F} \\
\Leftrightarrow \quad \mathrm{d}_{t} \vec{r} & =\left(\mathbb{1}-\zeta_{i} \mathbf{D} \cdot \mathbf{M}\right)^{-1} \cdot \mathbf{D} \cdot \vec{F} \\
\Leftrightarrow \quad \mathrm{d} \vec{r} & =: T^{-1} \hat{\boldsymbol{D}} \cdot \vec{F}^{\operatorname{det}} \mathrm{d} t+\sqrt{2} \hat{\boldsymbol{B}} \cdot \mathrm{d} \vec{W}
\end{aligned}
$$

where $\mathbf{M}$ consists of blocks $\mathbf{M}_{i, i+1}=\mathbf{M}_{i, i-1}=\rrbracket_{3 \times 3}, \mathbf{M}_{i i}=-2 \rrbracket_{3 \times 3}$ and is zero elsewhere, and $\vec{F}^{\text {det }}$ denotes the collection of all deterministic forces. Following this approach, one finds that the fluctuation-dissipation relation $\hat{\boldsymbol{D}}=\hat{\boldsymbol{B}} \cdot \hat{\boldsymbol{B}}^{T}$ is not fulfilled. This is not surprising because the fluctuation-dissipation theorem [152] states that any source of friction also causes thermal fluctuations (random forces), and here we have only heuristically added a friction force but no additional source of noise. To correct for this, I suggest replacing the matrix $\hat{\boldsymbol{B}}$ above with the Cholesky decomposition of $\hat{\boldsymbol{D}}$ such that the detailed balance condition is fulfilled and the Boltzmann distribution is sampled.

This updated model possesses the additional free parameter $\zeta_{i}$ which quantifies the strength of internal friction. Note that without internal friction, the viscosity $\eta$ only entered our model's dynamics via the time scale $t_{0} \propto \eta$ (see section 4.1, page 85), and thus all simulated times were automatically proportional to it. This no longer holds true in presence of internal friction, and thus it is common practice in literature to infer the parameter $\zeta_{i}$ from the viscosity dependence of time-related quantities. Within the RIF model, for instance, it is known that the reconfiguration time $\tau^{R}$ of a Gaussian chain with internal friction is given by the sum of two terms - the first is proportional to $\eta$, and the other to $\zeta_{i}$. Hence, the offset of $\lim _{\eta \rightarrow 0} \tau^{R}(\eta)$ yields information about the strength of internal friction, and is also experimentally accessible.

I suggest that we utilize the same sort of trick and estimate $\zeta_{i}$ from experimental measurements of the mean loop formation time $k_{+}^{-1}$ as a function of solvent viscosity $\eta$. Its offset should then be compared to a model-derived prediction in terms of $\zeta_{i}$.

\subsection{Secondary structures and twist}

Our polymer model may be expanded by taking into account possible remnants of secondary structures in the measured proteins. To do so, the constrained Langevin equation does not need to be altered at all - only the energy function $E$ does. As was explained in section 2.3.2 on page 61, we use an energy function from within the framework of discrete differential geometry. It is capable of encoding an arbitrary energetic minimum of the modeled elastic rod, which can be chosen to e.g. resemble an $\alpha$-helix. It should be noted that, by doing so, one does not model the microscopic mechanisms (interactions between the amino acids' side chains) which result in 
secondary structures at all. Instead, one inserts the result of these mechanisms into the model without specifying why there is e.g. an $\alpha$-helix. Such a procedure is obviously questionable at the level of microscopic physics, but it does serve a meaningful purpose: It allows us to investigate the impact of secondary structures on experimentally accessible observables; in particular on the function $k_{+}(s)$, i.e. the internal-to-end contact rate as a function of quencher position. In the absence of secondary structures the energetic minimum of the model polymer is a straight line, as it has been throughout this thesis. This led to $k_{+}(s)$ being a power law as long as $s$ is not too close to the free chain end such that the tail effect plays a role (see figure 5.6, page 114) and at least a few persistence lengths away from the fluorophore. In the presence of secondary structures, however, the functional form can be expected to be altered, and we currently lack a link between the two. During his doctoral work [130], my colleague Man Zhou worked with a short protein called the FSFG-protein and experimentally measured its function $k_{+}(s)$ (see figure 7.1). Its amino acid sequence is

\section{Atto655-CENSKTGFSFAPKSSDSDKKEDSKAGFSFAPKSADGDKKEDSKAGFSFAPK}

where the amino acids marked in red where replaced with tryptophan one at a time, or tryptophan was attached to the chain end.

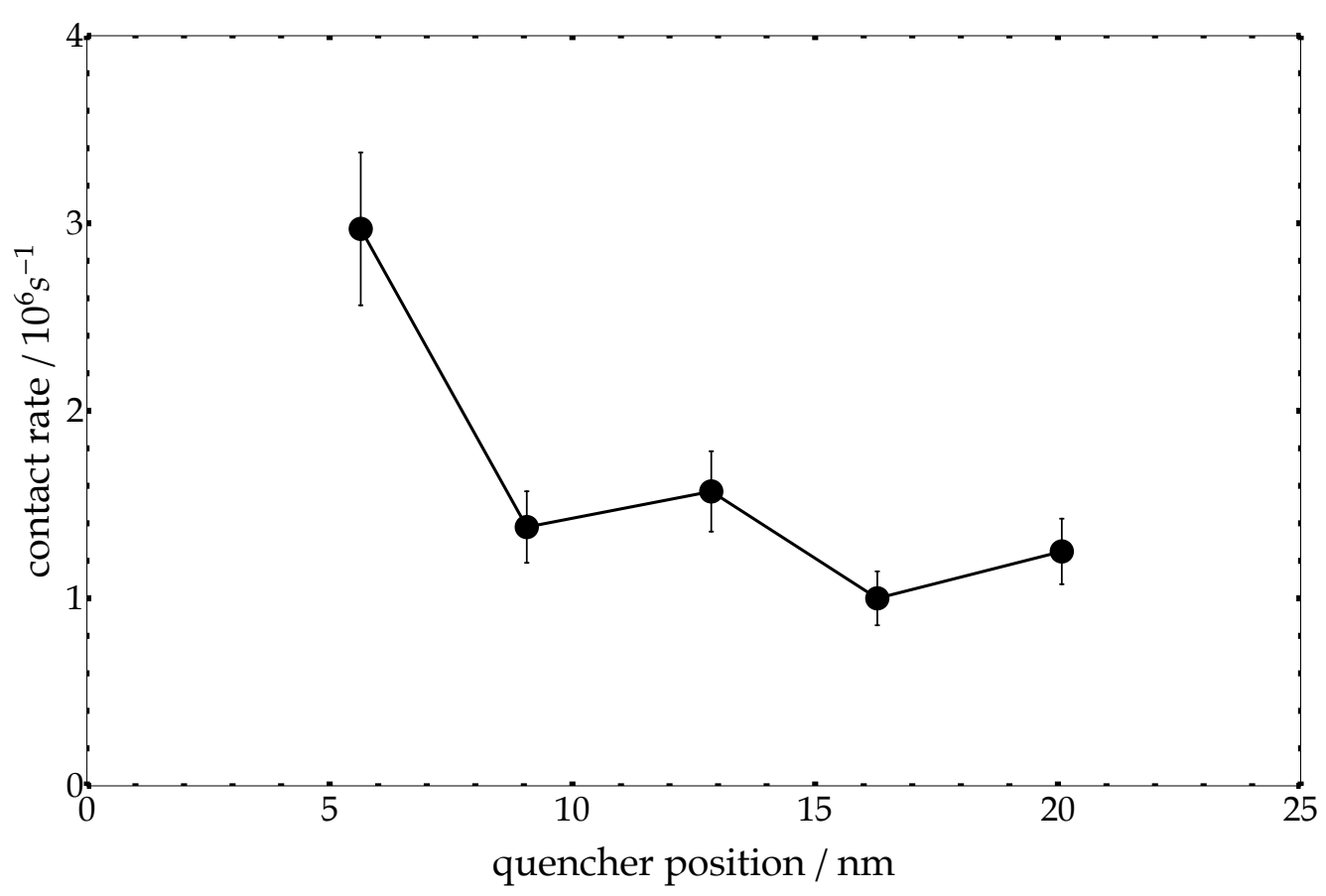

Figure 7.1: The internal-to-end contact rate measured in FSFG-proteins as a function of quencher position.

Interestingly, the measured function $k_{+}(s)$ is not strictly decreasing, but has a local maximum which cannot be explained with the current model. As a proof of principle, I have simulated 
$k_{+}(s)$ for a number of model chains with varying bending stiffness. All their reference curvatures encode two loops of a planar, circular ring such that the chain ends coincide with its center. The results as well as technical details of the simulations can be found in appendix G, where we also show that the influence of twisting elasticity on the end-to-end contact rate is very weak. It turns out that $k_{+}(s)$ can indeed have a local maximum when nonzero reference curvatures are introduced into our model. A detailed, quantitative analysis linking the shape and energetic stiffness of realistic secondary structures to the internal-to-end contact rates of the investigated proteins should be subject to future work, especially considering the unique vantage point which presents itself to us. As soon as such an analysis becomes available, it may become possible to combine our method with results of complementary methods quantifying the existence of secondary structures (circular dichroism [153] or Ramachandran plots [154]), or the free energy landscape (atomic force microscopy [155] or molecular dynamics simulations) in proteins.

\subsection{Repeat current protocol}

As explained in the previous two sections, our model can be extended to be more realistic, which comes at the cost of introducing additional fit parameters. However, the potential of the current model has not yet been fully exploited. The successful method of combining PET-FCS and 2fFCS experiments of end-to-end labeled homogeneous IDPs with our polymer model should be applied to other proteins. The amino acid sequences of those proteins should obviously differ from that of GS-repeats, but still be homogeneous. As a future goal, one may thereby obtain a list of bestfitting persistence lengths and hydrodynamic bead radii associated with different amino acids. Firstly, such a list would yield interesting insights, e.g. about which amino acid (or what chemical properties of amino acids) make proteins stiffer or diffuse faster than others do. Secondly, it would potentially allow us to tackle even inhomogeneous amino acid sequences by simulating a model polymer with parameters $a$ and $\sigma$ which vary along its contour according to a given experimental amino acid sequence.

Two prerequisites for our current method to be successful are that (i) the investigated protein does not fold but is intrinsically disordered, and (ii) that the amount of internal friction in it is negligible. As explained in the previous two sections, both criteria can be experimentally checked before the proteins are further investigated.

An ambitious future goal would be to have at hand a combined method of performing PET-FCS and 2fFCS experiments and numerical simulations within an extended polymer model, which not only delivers the measured proteins' persistence lengths and hydrodynamic properties, but also detects secondary structures and takes into account internal friction. 


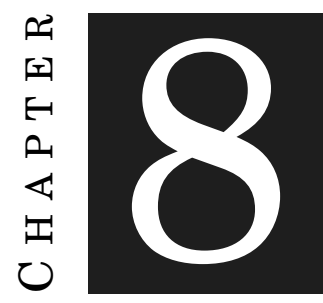

\section{OTHER CONTRIBUTIONS}

$\mathrm{D}$ uring my doctoral dissertation, I have worked on a number of side projects which I consider too unrelated to the main text of this thesis to be embedded into it. Nevertheless, all main and side projects share a common feature: They treat one-dimensional curves in threedimensional space. In the following, four projects will be presented in the following way: Firstly, the abstract of the published manuscript is presented, and the project's publication status and scientific context are briefly explained. Secondly, it is made clear what my own contribution to the project was, and selected parts of the published manuscript which are related to my own contribution are presented. The latter were slightly altered to better fit into this thesis, and are not meant as complete reproductions or summaries of the manuscripts. 


\title{
8.1 Geometric constraints in protein folding
}

\begin{abstract}
The intricate three-dimensional geometries of protein tertiary structures underlie protein function and emerge through a folding process from one-dimensional chains of amino acids. The exact spatial sequence and configuration of amino acids, the biochemical environment and the temporal sequence of distinct interactions yield a complex folding process that cannot yet be easily tracked for all proteins. To gain qualitative insights into the fundamental mechanisms behind the folding dynamics and generic features of the folded structure, we propose a simple model of structure formation that takes into account only fundamental geometric constraints and otherwise assumes randomly paired connections. We find that despite its simplicity, the model results in a network ensemble consistent with key overall features of the ensemble of Protein Residue Networks we obtained from more than 1000 biological protein geometries as available through the Protein Data Base. Specifically, the distribution of the number of interaction neighbors a unit (amino acid) has, the scaling of the structure's spatial extent with chain length, the eigenvalue spectrum and the scaling of the smallest relaxation time with chain length are all consistent between model and real proteins. These results indicate that geometric constraints alone may already account for a number of generic features of protein tertiary structures.
\end{abstract}

This project was published in PLOS ONE on February 27th 2020 [156], and is a collaboration with Nora Molkenthin, Antonia Mey and Marc Timme.

A well-known paradigm in biophysics is the protein-folding problem, which was described in the introduction of this thesis (see page 5) and the abstract above. In this work, we take a step back and identify universal features of folded protein structures which result from simple geometric constraints: any successful protein folding process has to (i) obey excluded volume interactions, while (ii) the backbone stays connected. To this end, we disregarded the heterogeneous nature of the protein's amino acid sequence and developed a simple geometric model which follows the same constraints: a chain of touching spheres. We repeatedly pull together two randomly chosen spheres of the chain, and permanently link them together if they could successfully be brought into contact while fulfilling the geometric constraints (including the maintenance of previously formed links). This process was repeated until no more links could be formed.

My contribution was to design, implement and run the simulation algorithm. What follows is the part of the published manuscript in which this algorithm is described. 
Selected part of published manuscript We have simulated the process modifying the chain geometry in 3D and tested the geometric constraints according to an algorithm consisting of repeated cycles of:

1. A pair $\left(i^{*}, j^{*}\right)$ of non-adjacent spheres is randomly chosen from the uniform distribution among the set of untried pairs.

2. The two spheres are attempted to be connected by switching on a force of unit strength pointing towards each other (see figure 8.1), under the geometric constraints:

(i) the backbone spheres stay together

(ii) no spheres overlap

(iii) spheres connected previously stay together.

3. If the selected spheres touch, a new link between them forms and we update the adjacency matrix ${ }^{1}$ by setting the elements $A_{i^{*} j^{*}}^{\operatorname{sim}}=A_{j^{*} i^{*}}^{\text {sim }}=1$. Alternatively, if the spheres move less than a velocity threshold $\Delta R / \Delta t$, the link is discarded and marked geometrically impossible (see below for details).

This process is repeated until no further link remains untried. During each cycle, to emulate the direct motion of spheres towards each other and to continuously match all geometric constraints, we change the spheres' positions $x_{i}, i \in\{1, \ldots, N\}$, according to simple overdamped dynamics

$$
\mathrm{d}_{t} x_{i}=\zeta F_{i}(x)
$$

where $x=\left(x_{1}, \ldots, x_{N}\right)^{T}$ is the collection of all positions and $F_{i}(x)$ is the sum of all constraint forces acting on sphere $i$ and, if $i \in\left\{i^{*}, j^{*}\right\}$, the unit force of magnitude 1 . The space and time scale were chosen such that all quantities are dimensionless, the single-sphere friction coefficient $\zeta$ is set to 1 and a distance of $x=1$ corresponds to a bond length between neighboring spheres.

The constraints are approximated by taking the total force

$$
F_{i}(x)=-\nabla_{i} V(x)+F_{i}^{\text {connect }}(x)
$$

as the sum of the forces inducing the connection attempt as

$$
F_{i}^{\text {connect }}(x)=\left(\delta_{i, i^{*}}-\delta_{i, j^{*}}\right) \frac{x_{j^{*}}-x_{i^{*}}}{\left|x_{j^{*}}-x_{i^{*}}\right|}
$$

and the constraint forces that are gradients of summed potentials

$$
V(x)=\frac{K}{2} \sum_{n, m=1}^{N} \frac{1}{2}\left(d_{n, m}-1\right)^{2}\left(A_{n m}^{\text {sim }}+\Theta\left(1-d_{n, m}\right)\right),
$$

\footnotetext{
${ }^{1}$ The $N \times N$ adjacency matrix $\mathbf{A}$ of a chain consisting of $N$ spheres has elements $A_{j i}$. These elements equal one if spheres $i$ and $j$ are linked together or are neighbors along the chain, and are zero otherwise.
} 
which are quadratic in the distances $d_{n, m}=\left|x_{n}-x_{m}\right|$. Here the Heaviside step function is defined as $\Theta(y)=0$ if $y<0$ and $\Theta(y)=1$ if $y \geq 0$. The first term in the final parenthesis in equation 8.3 ensures keeping neighboring units along the chain in contact as well as all other pairs of spheres linked so far during the process. The second term causes overlapping spheres to repel each other. $K$ is an elastic constant chosen large enough for the constraints to be virtually fulfilled and the final chain statistics being invariant of choosing larger values for $K$, but small enough in order not to limit the allowed numerical time steps unnecessarily. The value $K=50$ was found to meet these conditions and is used in simulations throughout the manuscript.

The initial configuration of the chain was drawn from a Boltzmann distribution with probability $p=Z^{-1} \exp \left(-E_{\text {Bend }} / k_{B} T\right)$ with $k_{B} T=1$ and energy

$$
E_{\text {Bend }}=-\kappa \sum_{n=2}^{N-1} \cos \left(\theta_{n}\right)
$$

where $Z$ is a normalization constant and $\theta_{n}$ is the bending angle at the $n$th unit of the chain, defined as the angle between the adjacent tangential vectors through the scalar (dot) product $\cos \theta_{n}=\left(x_{n}-x_{n-1}\right) \cdot\left(x_{n+1}-x_{n}\right)$, noting that the sphere diameter equals unity. Initially generated chains were rejected if any constraint was violated. The prefactor $k$ can be interpreted as bending stiffness and determines the persistence length of the initial chains. It was set to $\kappa=5$ such that initial chains are slightly bent (see figure 8.1 for an example).

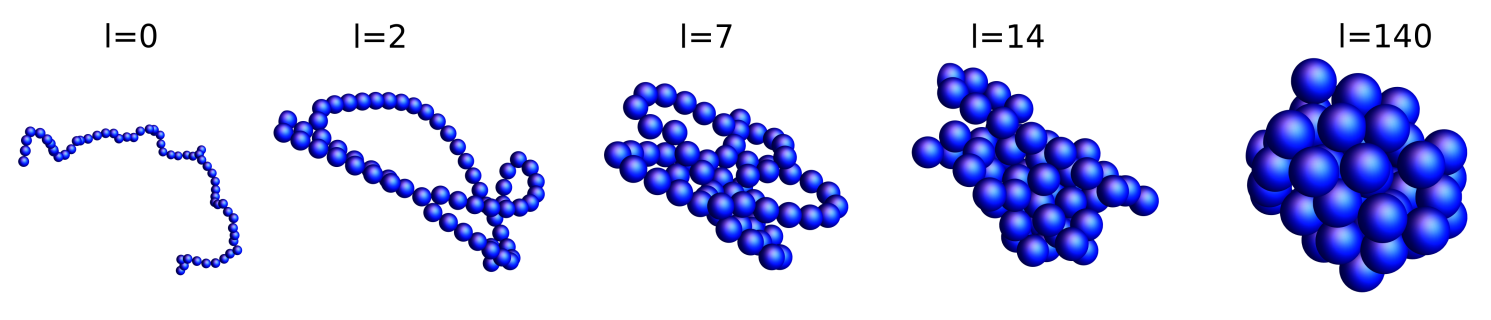

Figure 8.1: Model folding process at different times. Starting from an initial chain with $N=60$, randomly picked units connect if geometrically possible. Shown here are examples after $l=0,2,7,14$ and 140 successful connection attempts.

During a cycle started by selecting the spheres $i^{*}$ and $j^{*}$ to be pulled together, we monitored their decreasing distance $d_{i^{*}, j^{*}}$. As soon as $d_{i^{*}, j^{*}} \leq 1$, the cycle is considered successful and a new link is formed. We have also periodically checked at intervals $\Delta T$ whether $d_{i^{*}, j^{*}}$ has shrunk by less than a threshold distance $\Delta R=\Delta T \cdot \chi \cdot 2 /(N / 2)$. If this is the case, the cycle is discarded as unsuccessful, because the pair of units cannot make contact due to geometric constraints. The configuration at the beginning of that cycle is then restored. The last factor in $\Delta R$ is the relative velocity of the spheres $i^{*}$ and $j^{*}$ in case both - in order to move - have to drag half the other spheres $(N / 2)$ along. This lower velocity threshold was further decreased by introducing 


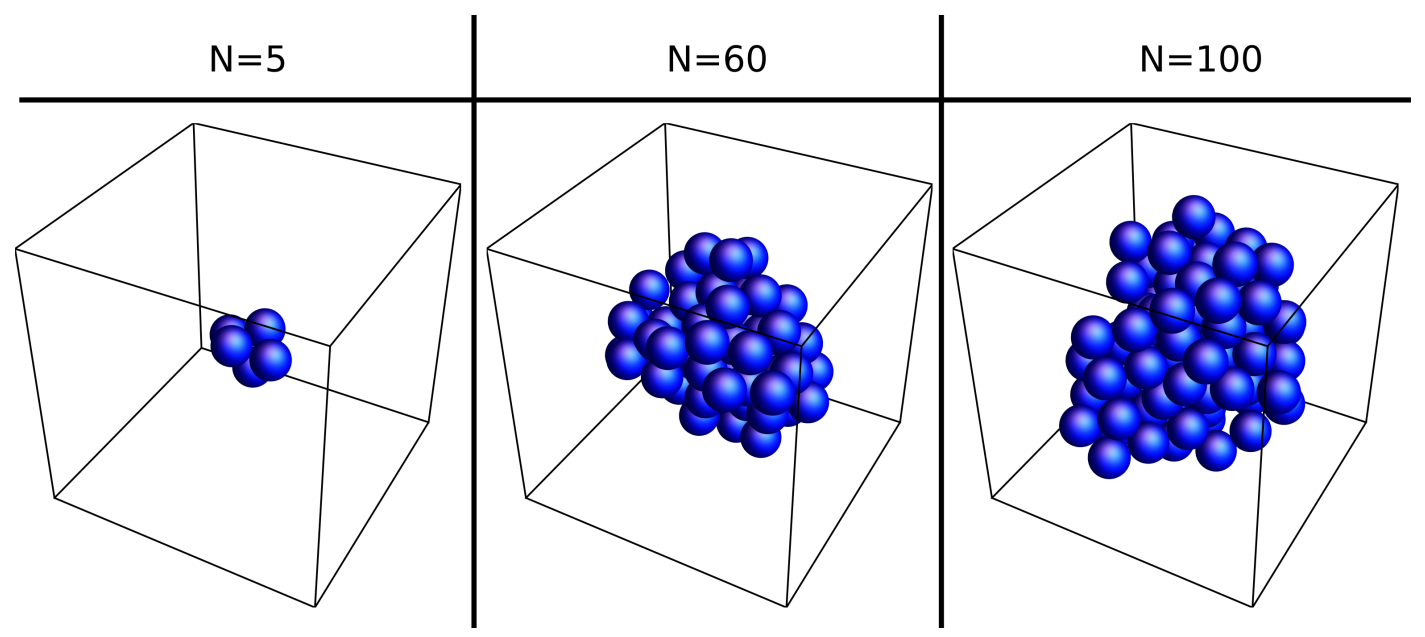

Figure 8.2: Final model aggregates. The final aggregates of the simulation for $N \in\{5,60,100\}$ display the expected compactness.

the factor $\chi=0.3$ because the final chain statistics weakly varied for larger values but remained the same for smaller values. We have found $\Delta T=0.15$ to be small enough in order not to waste computational time on unsuccessful cycles, but large enough to not abort cycles in which $d_{i^{*}, j^{*}}$ shrinks slowly only temporarily.

The excluded volume forces are nonzero only for pairs of spheres whose distance is less than one. To speed up the simulation, they were only evaluated for spheres that are elements of each others neighbor list listing all spheres within a distance $1+\epsilon$. We initially generated these lists, then integrated the maximum velocity of all spheres over time and updated the neighbor lists whenever the resulting value exceeded $\epsilon$. The value $\epsilon=0.2$ provided the best speed-up. At each integer multiple of 100 cycles, all untried links to a sphere $i$ with $\sum_{j=1}^{N} A_{i j}^{\text {sim }}=: k_{i} \geq 12$ were discarded. This measure was taken to accelerate the simulations as further bonding trials including this sphere are geometrically impossible.

As the next step, the simulated final configurations' network structures as well as their radii of gyration were compared with those of real proteins extracted from the Protein Data Base. For further reading, we refer to the published manuscript. 


\title{
8.2 Multiplane imaging of flagellar motion
}

\begin{abstract}
Cilia and flagella are whip-like cellular appendages found from microorganisms to large animals. Motile ciliary structures are mainly responsible for cell locomotion and generation of fluid flow across surfaces of cells and tissues. Considerable efforts have been invested to elucidate the mechanics and dynamics of cilia and flagella. But only recently it became clear that three-dimensional models and corresponding experiments are required for understanding their motion and mechanics. Flagella of micro-organisms such as Chlamydomonas reinhardtii are small (length $\sim 10 \mu \mathrm{m}$, diameter $\sim 0.15 \mu \mathrm{m})$ and beat rapidly $(\sim 50 \mathrm{~Hz})$. This makes the recording of their three-dimensional dynamics difficult. Here, we use a new multi-plane phase-contrast microscope for observing the three-dimensional beating behavior of unlabeled Chlamydomonas flagella with diffraction-limited resolution and several hundred frames per second. We find two types of flagellar motion: one type swims in counter-clockwise circles close to an interface and exhibits negative torsion values at their basal end. The much rarer second type shows reversed swimming direction and positive torsion values. We explain the signature of the torsion by the structural chirality of the axoneme, and thus directly connect axonemal structure with macroscopic swimming behavior. Our study is important for theoretical modeling of axoneme mechanics, and calls for future structural studies of the predicted connection between structure and swimming dynamics.
\end{abstract}

This project was submitted to Physical Review $X$ on May 27th 2020, and is a collaboration with Soheil Mojiri (experimental work + data analysis), Azam Gholami (sample preparation), Sebastian Isbaner (tracking + data analysis), Hongje Jang (data deconvolution + analysis), Jörg Enderlein (project coordination + data analysis) and Ingo Gregor (help with experiments).

We studied the three-dimensional dynamics of flagella. Flagella are whip-like appendices of cells (both bacteria and eukaryotic cells) whose major biological function is that of swimming, but they also serve other purposes such as sensing outside chemicals or temperature. They may be thought of as the "arms" with which bacteria swim, and are essentially one-dimensional with a contour length of around $10 \mu \mathrm{m}$ and a diameter around $\sim 0.15 \mu \mathrm{m}$. Their cross section is a pillar-like structure which consists of two central fused-pairs of microtubules and nine outside pairs, between which dyneins are assembled. Dyneins are motor-proteins which convert chemical energy into physical work ${ }^{2}$ and can be thought of as the "engines" driving flagellar motion.

\footnotetext{
${ }^{2}$ They convert adenosintriphosphat (ATP) into adenosindiphosphat (ADP) - an exothermal reaction which allows them to assume conformational states of higher free energy (thus performing "a step").
} 
This inner structure of flagella (also called the "axoneme") is highly preserved throughout evolution and well-understood today. The relationship between dynein activity and flagellar motion is less well-understood, and here we study the latter from a purely descriptive point of view. The main novelty is that we can study the flagella in three spatial dimensions and can thus test whether their beat patterns are planar or not.

My contribution I have fitted continuous curves to experimentally tracked 3D data points which were provided to me, and thereby reconstructed axonemal shapes from noisy experimental data.

Based on the experimentally measured intensity profile (measured by Soheil Mojiri), a tracking routine (performed by Sebastian Isbaner) yielded $N=30$ data points $\vec{r}_{i}=\left(x_{i}, y_{i}, z_{i}\right)$ which approximate an axoneme as a polygonal chain in three dimensions. The tracking routine was applied to all time frames in all measured axonemes, and thus we have at hand movies of discretized axonemes. Due to the presence of noise in the experimental data, these discrete shapes are far from "smooth" and are nontrivial to analyze: discrete curvature values as well as torsion estimators often yield extreme noise-dominated values. In order to retrieve and analyze the axonemes' shapes, we fit continuous, smooth curves to the polygonal chains. This is done as follows: The continuous curve $\vec{r}(s)$, where $s$ is its arc length, is described by a Bishop frame as we have introduced in section 2.3.1 (see page 55) of this thesis. Their shape is hence fully described by the complex curvature $\Psi(s)$. We represent both the real and imaginary part of $\Psi$ as a sum of $M$ modes of base functions $z_{k}(s)$ and, by introducing the complex mode coefficients $c_{k}$, write

$$
\Psi(s)=\sum_{k=1}^{M} c_{k} z_{k}(s) .
$$

We have chosen Chandrasekhar modes [157] for the base functions $z_{k}(s)$. They fulfill the differential equation $\partial_{s}^{4} z \propto z$, the boundary conditions $\left.z\right|_{0,1}=\left.\partial_{s} z\right|_{0,1}=0$ and are orthonormal with respect to the standard $L_{2}$ norm. The first few Chandrasekhar modes are displayed in figure 8.3 and their functional form is given by equation 8.8 , which we remark on by the end of this section.

These boundary conditions (BC) are those of a freely swimming elastic rod [78], but we emphasize that we only use them for the practical purpose of curve fitting and do not make assumptions about the axonemes' mechanics. The used BC make the fitted curve's curvature and its derivative vanish at its ends. Therefore the fitted curve cannot overfit single data points at the polygon's two ends. Using Fourier modes instead (thereby not imposing any boundary conditions at all) yielded occasional overfitting artifacts at the boundaries but otherwise similar results. Additionally, we observed a better fit quality for Chandrasekhar modes than for Fourier modes when the same number of free parameters was used. For BC reflecting the full, active mechanics of axonemal motion, we refer to reference [158]. 


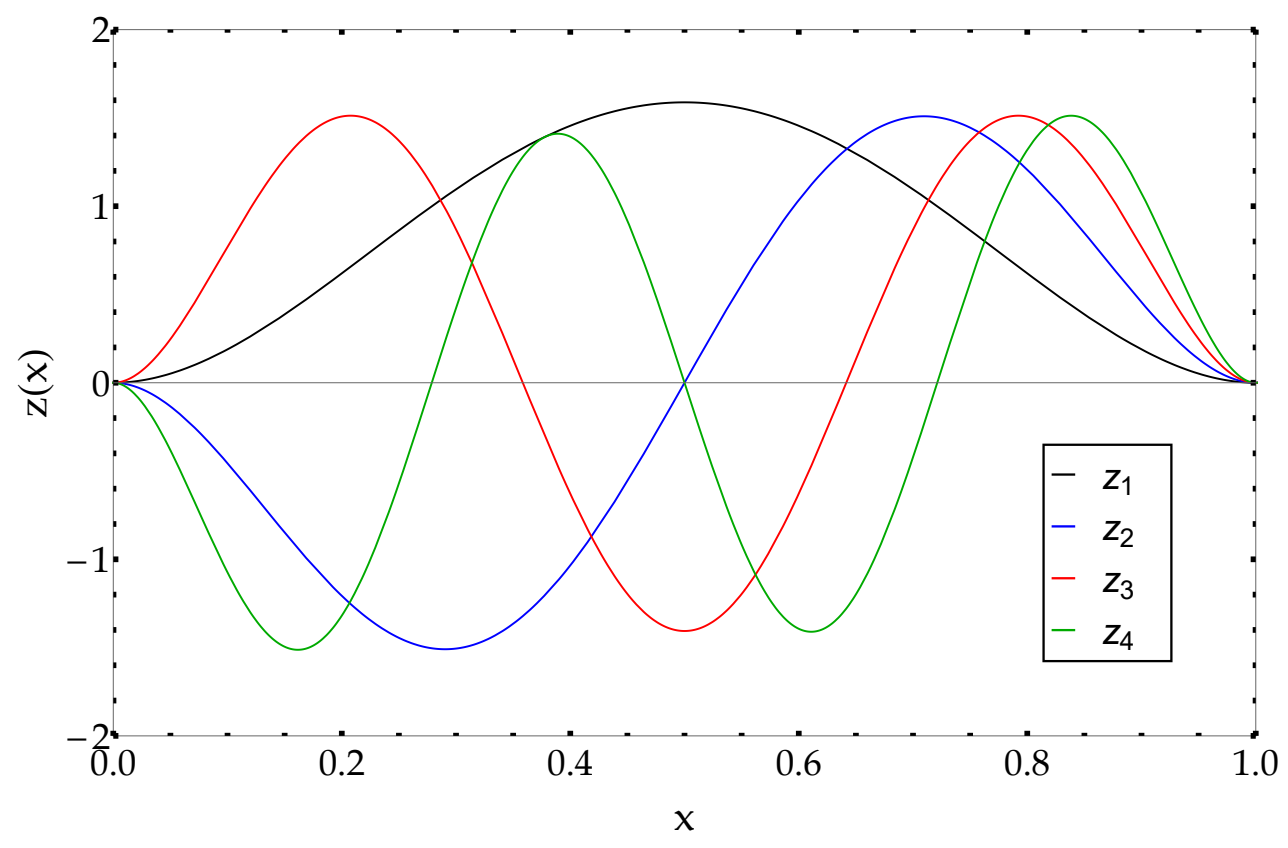

Figure 8.3: The first four Chandrasekhar modes.

For the initial conditions of the Bishop frame at $s=0$ only 2 additional free parameters are required due to the orthonormality of the frame. They determine the direction of the tangent vector (e.g. in spherical coordinates). The rotational angle of the initial triad around its tangential direction is redundant with globally changing the complex phase of $\Psi$, and hence it does not constitute another fit parameter. We chose the initial point $\vec{r}_{0}=\vec{r}(s=0)$ where the fitted curve starts such that the centers of mass of the fit curve and that of the 30 tracked data points always coincide, thereby eliminating the components of $\vec{r}_{0}$ as free parameters. The last fit parameter is the curve's contour length $L$, and hence the total number of free parameters is $2 \cdot M+3$. The number of modes, $M$, was chosen such that the axoneme's shape dictated by the tracked data points was well captured, but not overfitted. Furthermore, because Chandrasekhar modes $z_{k}(s)$ are alternatingly even and odd functions with respect to the axoneme's center, $M$ should be an even number in order to capture the symmetric and asymmetric parts of the true $\Psi$ equally well. These requirements were met by using $M=4$ complex modes. The collection of fit parameters, $\vec{p}$, was chosen such that the error function

$$
E(\vec{p})=\sum_{i=1}^{30}\left(\vec{r}\left(s=\frac{i-1}{29} L\right)-\vec{r}_{i}\right)^{2}
$$

was minimized. In figure 8.4, two representative fits are shown. The fit quality could be further increased by increasing the number of modes $M$. This would result in overfitting of experimental noise, however, and the whole point of fitting continuous curves was to "smooth" out outliers from individual data points. 

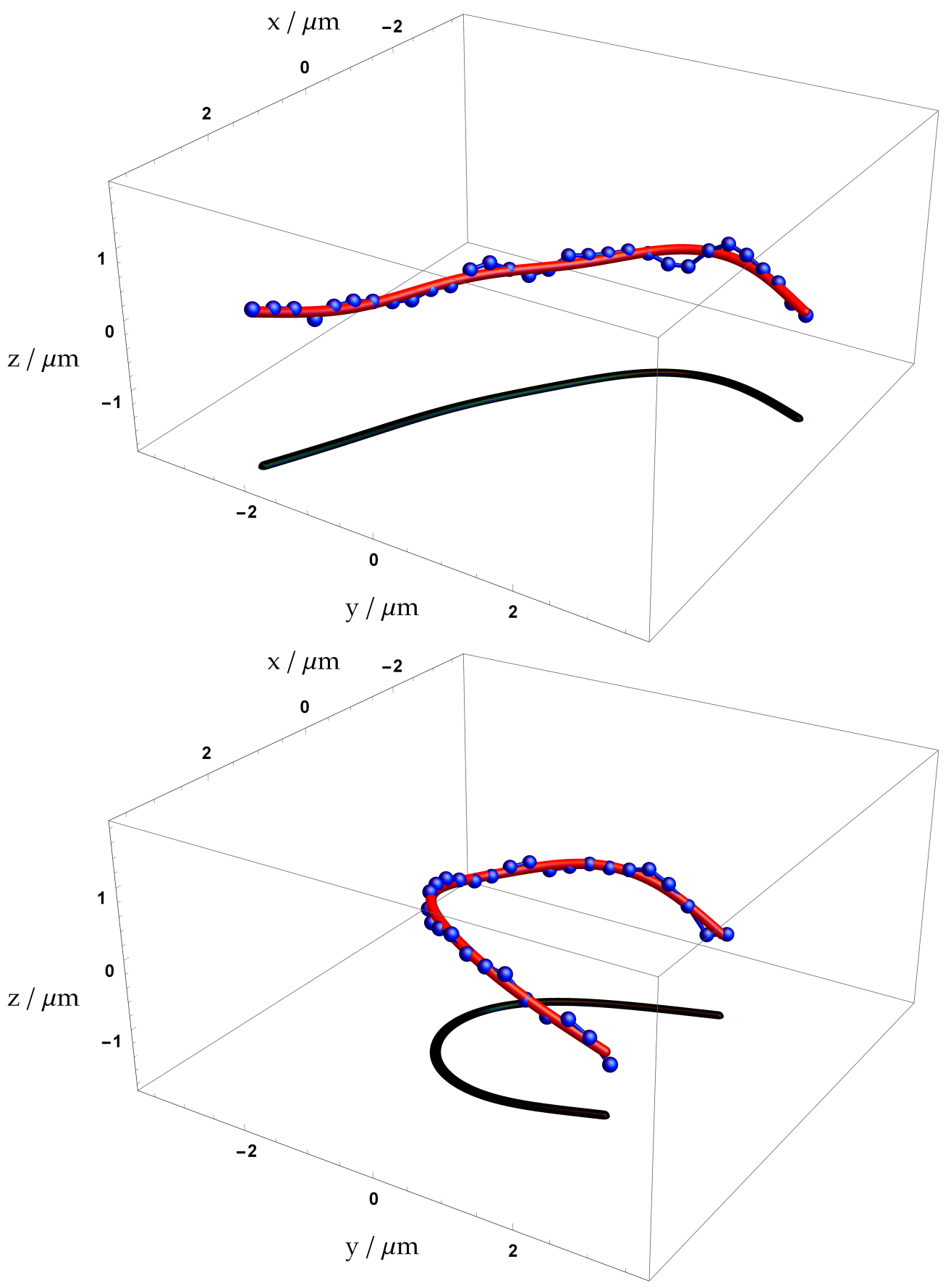

Figure 8.4: Two representative fitted axonemes. The blue data points result from applying a tracking routine to the experimentally measured 3D intensity profile. The red curves are the reconstructed shapes from our fitting routine. The black shape in the $x$-y-plane is a projection (shadow) of the fitted curve.

Next, the fitted shapes' curvature and torsion were analyzed as functions of arc length and time. For further reading, we refer to the submitted manuscript. 
About Chandrasekhar functions An important result one finds when analyzing the response of an elastic rod to bending is that boundary conditions apply to the complex curvature $\Psi$. In order to briefly demonstrate the boundary conditions' origin, let us calculate the variation of the elastic bending energy $E$ (see section 2.3.1, page 54):

$$
\begin{aligned}
\delta E & =\delta \frac{\alpha}{2} \int|\Psi|^{2} \mathrm{~d} s^{\prime}=\delta \frac{\alpha}{2} \int\left|\partial_{s}^{2} \vec{r}\right|^{2} \mathrm{~d} s^{\prime} \\
& =\int \alpha\left(\partial_{s}^{2} \vec{r} \cdot \delta \partial_{s}^{2} \vec{r}\right) \mathrm{d} s \\
& =\int \alpha\left(\partial_{s}^{2} \vec{r} \cdot \partial_{s}^{2} \delta \vec{r}\right) \mathrm{d} s \\
& =\int \alpha\left(\partial_{s}^{4} \vec{r} \cdot \delta \vec{r}\right) \mathrm{d} s
\end{aligned}
$$

The force density $-\delta E / \delta \vec{r}$ thus equals the fourth spatial derivative of $\vec{r}$. In the last line we have partially integrated twice and thereby made use of the boundary conditions $\partial^{2} \vec{r} / \partial s^{2}=\partial^{3} \vec{r} / \partial s^{3}=0$ which immediately leads to $\Psi=\Psi_{s}=0$. In the calculation above, we have assumed inextensibility of line segments $(\delta d s=0)$. A more thorough treatment is available in literature [78, 112].

A set of functions $z_{k}(s)$ which completely spans the space of functions satisfying $z_{k}(0)=\partial_{s} z_{k}(0)=$ $z_{k}(L)=\partial_{s} z_{k}(L)=0$ are called the Chandrasekhar functions [157] and they read

$$
z_{k}(s)= \begin{cases}\frac{\cosh \left(\lambda_{p}(s / L-0.5)\right)}{\cosh \left(\lambda_{p}\right)}-\frac{\cos \left(\lambda_{p}(s / L-0.5)\right)}{\cos \left(\lambda_{p} / 2\right)} & \text { for even } \mathrm{k} \\ \frac{\sinh \left(\mu_{q}(s / L-0.5)\right)}{\sinh \left(\mu_{q} / 2\right)}-\frac{\sin \left(\mu_{q}(s / L-0.5)\right)}{\sin \left(\mu_{q} / 2\right)} & \text { for odd } \mathrm{k}\end{cases}
$$

where $p=k / 2, q=(k+1) / 2$ and the coefficients $\lambda$ and $\mu$ are the solutions of $\tanh (\lambda / 2)+\tan (\lambda / 2)=0$ and $\tanh (\mu / 2)-\tan (\mu / 2)=0$ respectively. The Chandrasekhar functions satisfy the characteristic equation

$$
\partial_{s}^{4} z_{k} \propto z_{k}
$$

and are thus eigenfunctions of the fourth-derivative differential operator. The first few Chandrasekhar modes are shown in figure 8.3. 


\title{
8.3 Electric field lines of an arbitrarily moving point charge
}

\begin{abstract}
Generation of electromagnetic fields by moving charges is a fascinating topic where the tight connection between classical electrodynamics and special relativity becomes particularly apparent. One can gain direct insight into the fascinating structure of such fields by visualizing the electric field lines. However, the calculation of electric field lines for arbitrarily moving charges is far from trivial. Here, we derive an equation for the director that points from the retarded position of a moving charge towards a specific field line position, which allows for a simple construction of these lines. We analytically solve this equation for several special but important cases: for an arbitrary rectilinear motion, for the motion within the wiggler magnetic field of a free electron laser, and for the motion in a synchrotron.
\end{abstract}

This project was published in the American Journal of Physics on December 23rd 2019 [159]. The research for it was done by Jörg Enderlein and Daja Ruhland.

I have merely helped by revising the manuscript, double-checking calculations and making the presented line of arguments more consistent.

Selected part of published manuscript Electric and magnetic fields generated by arbitrarily moving point charges are a fascinating topic where relativistic physics meets classical electrodynamics. In particular, accelerated point charges are the generators for almost all electromagnetic radiation, such as that emitted by oscillating electric dipoles, synchrotrons, or free electron lasers. As is well known, the electric field $\vec{E}$ of an arbitrarily moving point charge $q$ can be found with the help of Liénard-Wiechert potentials and has the explicit form [160, 161]

$$
\vec{E}(\vec{r}, t)=q\left\{\frac{\vec{R}-R \vec{\beta}}{\gamma^{2}(R-\vec{R} \cdot \vec{\beta})^{3}}+\frac{\vec{R} \times[(\vec{R}-R \vec{\beta}) \times \dot{\vec{\beta}}]}{c(R-\vec{R} \cdot \vec{\beta})^{3}}\right\}_{t^{\prime}}
$$

where the three-dimensional vector $\vec{R}$ is the spatial part of the four-dimensional null-vector

$$
\left\{c\left(t-t^{\prime}\right), \vec{r}-\vec{r}_{0}\left(t^{\prime}\right)\right\}
$$

This null-vector defines the retarded time $t^{\prime}<t$ via

$$
t-t^{\prime}=\frac{R}{c}=\frac{\left|\vec{r}-\vec{r}_{0}\left(t^{\prime}\right)\right|}{c}
$$

at which the right hand side of equation 8.10 has to be evaluated. Here, $\vec{r}_{0}(t)$ is the particle's trajectory as a function of time $t$. Furthermore, the symbol $\vec{\beta}(t)=c^{-1} \mathrm{~d}_{t} \vec{r}_{0}(t)$ is the particle's velocity divided by the speed of light $c, \gamma$ is the usual Lorentz factor $\gamma=1 / \sqrt{1-\beta^{2}}$, and a dot denotes differentiation with respect to time. For finding the electric field at a given position $\vec{r}$ and 


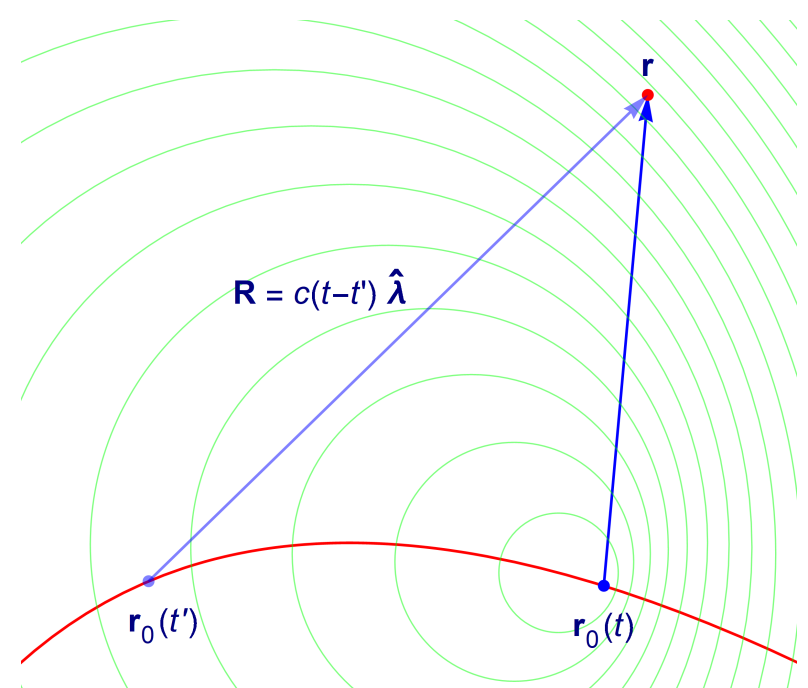

Figure 8.5: A point charge moving along an arbitrary trajectory $\vec{r}_{0}(t)$ (red solid line) generates an electromagnetic field throughout space. The field at any given position $\vec{r}$ at time $t$ originates from the charge when it is at retarded position $\vec{r}_{0}\left(t^{\prime}\right)$. Green circles are lines of constant retarded time $t^{\prime}$. The vector $\vec{R}=c\left(t-t^{\prime}\right) \hat{\vec{\lambda}}$ is the spatial part of the null-vector of equation 8.11 which connects the space-time events $\left\{c t^{\prime}, \vec{r}_{0}\left(t^{\prime}\right)\right\}$ and $\{c t, \vec{r}\}$, so that $\hat{\vec{\lambda}}$ is a unit vector.

time $t$, one has firstly to solve the retarded time equation 8.12 , and then secondly to evaluate the right hand side of equation 8.10 at time $t^{\prime}$, which is typically a numerically demanding task.

Another way of visualizing an electric field is to use electric field lines - continuous lines tangential to the electric field vector. Visualization of field lines can help in better understanding complex field configurations generated by non-trivial particle trajectories, and knowledge of field lines can also be used to estimate the electric field strength, due to the interconnection between local field line density and field strength as embodied by the zero divergence of the electric field in source-free space. Thus, the question how to efficiently calculate and draw field lines for arbitrarily moving point charges has been repeatedly considered in the literature [162-169]. Here, we present an efficient and relatively simple way of how to find and draw electric field lines of an arbitrarily moving charge by deriving a compact auxiliary equation for a unit vector pointing from the retarded position of the charge to a specific field line position. We then find analytic solutions of the problem for several important cases.

Let us describe a field line at time $t$ by a parametric three-dimensional curve $\vec{p}(s)$ which is parametrized by the variable $s$. Along all its positions, it has to be parallel to the electric field vector, which means that it has to obey the differential equation

$$
\frac{d \vec{p}(s)}{d s} \propto \vec{E}[\vec{p}(s)]
$$


Taking into account the non-trivial form of the electric field as given by equation 8.10, finding analytic solutions to this equation seems to be a formidable task. Note that any Cartesian position $\vec{r}$ can geometrically be referenced to the retarded position $\vec{r}_{0}\left(t^{\prime}\right)$ by $\vec{r}=\vec{r}_{0}\left(t^{\prime}\right)+\vec{R}\left(t^{\prime}\right)$, where $t^{\prime}$ is the retarded time of the particle's position when it contributes to the electric field at position $\vec{r}$, see also figure 8.5. In particular, this holds true also for positions $\vec{r}=\vec{p}(s)$ on a field line. Our core idea is to use the retarded time $t^{\prime}$ to parametrize a field line, by setting $s=t^{\prime}$. Thus, the time $t^{\prime}$ has a double meaning: it denotes the retarded time $t^{\prime}$ and it parametrizes the field line, and we find for the field line positions the relation

$$
\vec{p}\left(t^{\prime}\right)=\vec{r}_{0}\left(t^{\prime}\right)+\vec{R}\left(t^{\prime}\right)=\vec{r}_{0}\left(t^{\prime}\right)+c\left(t-t^{\prime}\right) \hat{\vec{\lambda}}\left(t^{\prime}\right)
$$

where we have used the fact that the length of the vector $\vec{R}\left(t^{\prime}\right)$ is $c\left(t-t^{\prime}\right)$, so that the vector $\hat{\vec{\lambda}}\left(t^{\prime}\right)$ on the right hand side of equation 8.14 is a unit vector pointing from the retarded position $\vec{r}_{0}\left(t^{\prime}\right)$ of the point charge to a position $\vec{p}\left(t^{\prime}\right)$. Now, let us consider equation 8.13. Because we require that vector $d \vec{p} / d s \equiv d \vec{p} / d t^{\prime}$ and vector $\vec{E}$ have only to be parallel at all positions $\vec{p}\left(t^{\prime}\right)$, we can choose any proportionality factor in equation 8.13 between these two vectors. Let us thus set $d \vec{p} / d t^{\prime}$ equal to $-c \gamma^{2} R^{2}(1-\hat{\vec{R}} \cdot \vec{\beta})^{3} \vec{E} / q$ so that we find the field-line determining equation

$$
\frac{d \vec{p}\left(t^{\prime}\right)}{d t^{\prime}}=-c(\hat{\vec{R}}-\vec{\beta})-\gamma^{2} \vec{R} \times[(\hat{\vec{R}}-\vec{\beta}) \times \dot{\vec{\beta}}]
$$

where a hat over a vector symbolizes normalization (unit vector). Now, by inserting equation 8.14 into the last equation, we find the following auxiliary equation for the unit vector $\hat{\vec{\lambda}}\left(t^{\prime}\right)=\hat{\vec{R}}$

$$
\frac{d \hat{\vec{\lambda}}}{d t^{\prime}}=\gamma^{2}[(\hat{\vec{\lambda}}-\vec{\beta}) \times \dot{\vec{\beta}}] \times \hat{\vec{\lambda}}
$$

This equation is the core result of our paper: When we can solve this equation and determine $\hat{\vec{\lambda}}\left(t^{\prime}\right)$ for all times $t^{\prime}<t$, then we can use equation 8.14 to find the full field line. Thus, $t^{\prime}$ plays the role of a line parameter and does not have to be found a priori from an implicit retarded time equation such as equation 8.12 , as has to be done when calculating the electric field. The final condition of equation 8.16, i.e. the direction $\hat{\vec{\lambda}}\left(t^{\prime}=t\right)$, defines into which direction a field line starts from a point charge at time $t$.

Although we cannot present a general solution of equation 8.16 for an arbitrary motion $\vec{r}_{0}\left(t^{\prime}\right)$, we consider in the next chapters several important and quite general cases for which analytical solutions can be found.

As a first application of equation 8.16 we consider the well-known classical example of a uniformly accelerated charge [170] which is at rest at time zero, then (relativistically) accelerates along the (horizontal) $x$-direction with constant acceleration to the speed $c / \sqrt{2}$ within one unit of time, and then continues to move uniformly with that constant velocity. 


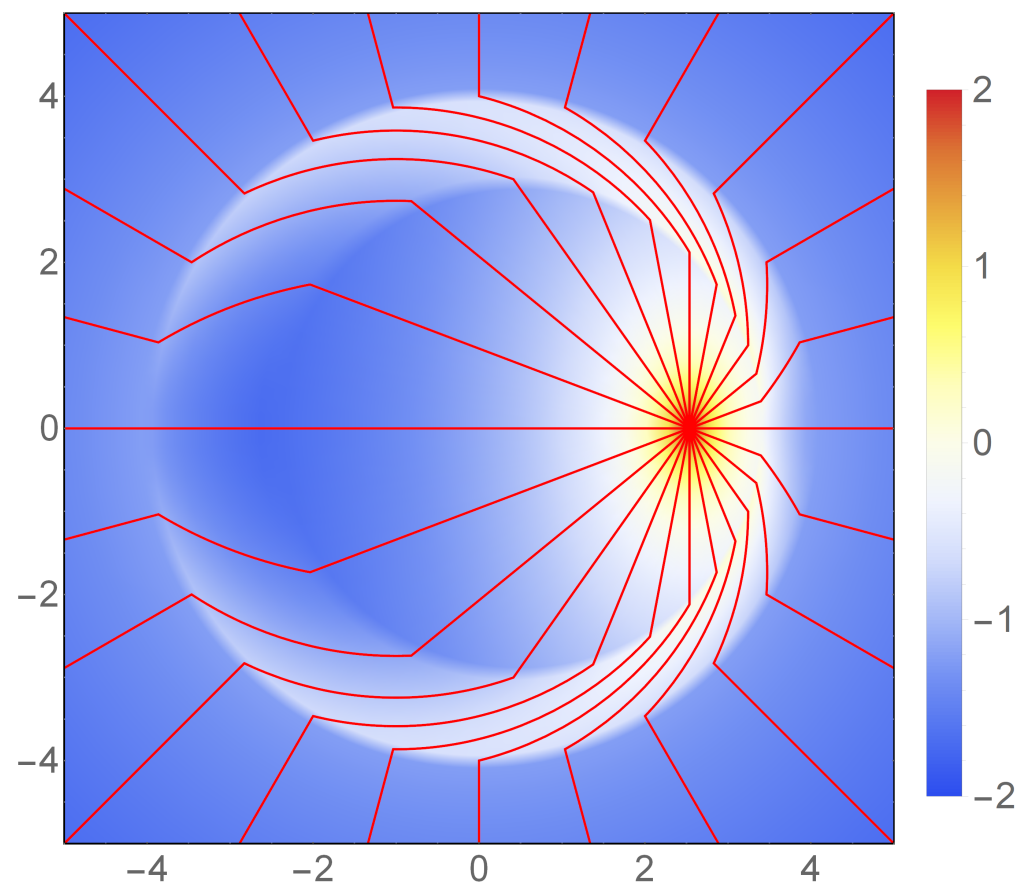

Figure 8.6: Electric field (density plot) and electric field lines (red) for an accelerated point charge. Its position along the horizontal axis ( $x$-axis) is given by equation 8.17 . The shown picture refers to time $t=4$, and the unit of length is chosen in such a way that the numerical value of the speed of light is one. The coloring encodes the decadic logarithm of the electric field amplitude $|\vec{E}|$ in arbitrary units. Here, we show field lines that start, in the particle's rest frame, from its position at angles $\phi=15^{\circ}$ to $\phi=360^{\circ}$ with respect to the horizontal axis in steps of $15^{\circ}$.

For such a motion, the particle's $x$-position as a function of time is given by

$$
x_{0}(t)=\left\{\begin{array}{ll}
0, & \text { if } t \leq 0 \\
c\left(\sqrt{1+t^{2}}-1\right), & \text { if } 0<t \leq 1 \\
c(\sqrt{2}-1+(t-1) / \sqrt{2}), & \text { if } t>1
\end{array} .\right.
$$

The resulting field line structure is displayed in figure 8.6. More applications - i.e. field lines resulting from different point charge trajectories than the one above - can be found in the published manuscript. 


\title{
8.4 Kinetics of loop closure in disordered proteins
}

\begin{abstract}
We study intrachain dynamics of intrinsically disordered proteins, as manifested by the time scales of loop formation, using atomistic simulations, experimentparametrized coarse-grained models, and one-dimensional theories assuming Markov or non-Markov dynamics along the reaction coordinate. Despite the generally nonMarkov character of monomer dynamics in polymers, we find that the simplest model of one-dimensional diffusion along the reaction coordinate (equated to the distance between the loop-forming monomers) well captures the mean first passage times to loop closure measured in coarse-grained and atomistic simulations, which, in turn, agree with the experimental values. This justifies use of the one-dimensional diffusion model in interpretation of experimental data. At the same time, the transition path times for loop closure in longer polypeptide chains show significant non-Markov effects; at intermediate times, these effects are better captured by the generalized Langevin equation model. At long times, however, atomistic simulations predict long tails in the distributions of transition path times, which are at odds with both the one-dimensional diffusion model and the generalized Langevin equation model.
\end{abstract}

This project was published in the Journal of Physical Chemistry B on April 7th 2020 [171]. The research for it was done by Rohit Satija and Dmitrii Makarov. My contribution was to run and make available end-to-end trajectories extracted from the polymer model developed in this thesis, and to help in revising the manuscript (as did Jörg Enderlein and Atanu Das). Atanu Das has further made available end-to-end trajectories from MD simulations of GS-repeats.

In this project, the end-to-end dynamics in GS-repeats are compared from different viewpoints: Experimentally measured loop formation rates and trajectories from Brownian dynamics (BD) and molecular dynamics (MD) simulations are compared with (i) one-dimensional Markovian diffusion and (ii) one-dimensional non-Markovian diffusion. Trajectories from our Brownian dynamics model are particularly valuable in this context because - unlike other coarse-grained polymer models - it is specifically parametrized to match experimental data for GS-repeats and unlike experimental results - yields complete trajectories and not just contact rate values.

From the perspective of this thesis, the most interesting result of this project is that onedimensional descriptions fail in reproducing certain dynamical features of the end-to-end dynamics described by the BD and MD simulations. Nevertheless, the mean first passage time (inverse contact rate) is reasonably well captured by the one-dimensional picture. This match is in agreement with our finding that SSS-theory (which assumes one-dimensional Markovian end-to-end dynamics, see page 142) correctly predicts the scaling behavior of the contact rate as a function of chain length. For further reading, we refer to the published manuscript [171]. 



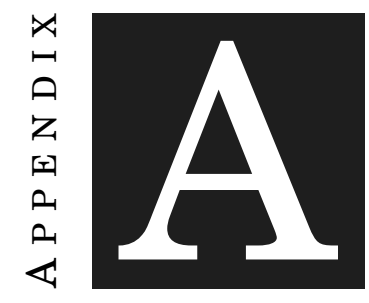

\section{Convergence OF Discrete CuRvatures TO THE WORM-LIKE CHAIN}

The context of this appendix is explained in section 2.3.2 on page 60. Here, we consider the convergence of the end-to-end distance's probability distribution in discretized worm-like chains towards the continuous limit. This convergence is being compared between three definitions of discrete curvature, which are

$$
\begin{aligned}
\kappa_{1} & =\frac{2 \tan \theta / 2}{\Delta s}, \\
\kappa_{2} & =\frac{2 \sin \theta / 2}{\Delta s} \\
\text { and } \quad \kappa_{3} & =\frac{\theta}{\Delta s}
\end{aligned}
$$

where $\theta$ is the bending angle at the considered chain vertex and $\Delta s$ is the arc length associated with it. For all chains considered here, the contour length equals 1 , and the persistence length equals $1 / 3$. The corresponding bending stiffnesses of the discrete chains were chosen according to equations 2.181 and 2.182 (page 68) for all discretizations and curvature definitions respectively. The probability distributions were obtained as histograms using MC sampling. An analytical approximation for the "true" end-to-end probability distribution in a continuous worm-like chain was taken from reference [172] whose author's made available a Mathematica notebook, which I have used. The following three figures show this analytical approximation and the numerically sampled probability distributions for chains consisting of $N \in\{5,10,20,40\}$ vertices.

It turns out that the tangent-based curvature definition yields the fastest convergence towards the continuous worm-like chain limit. 


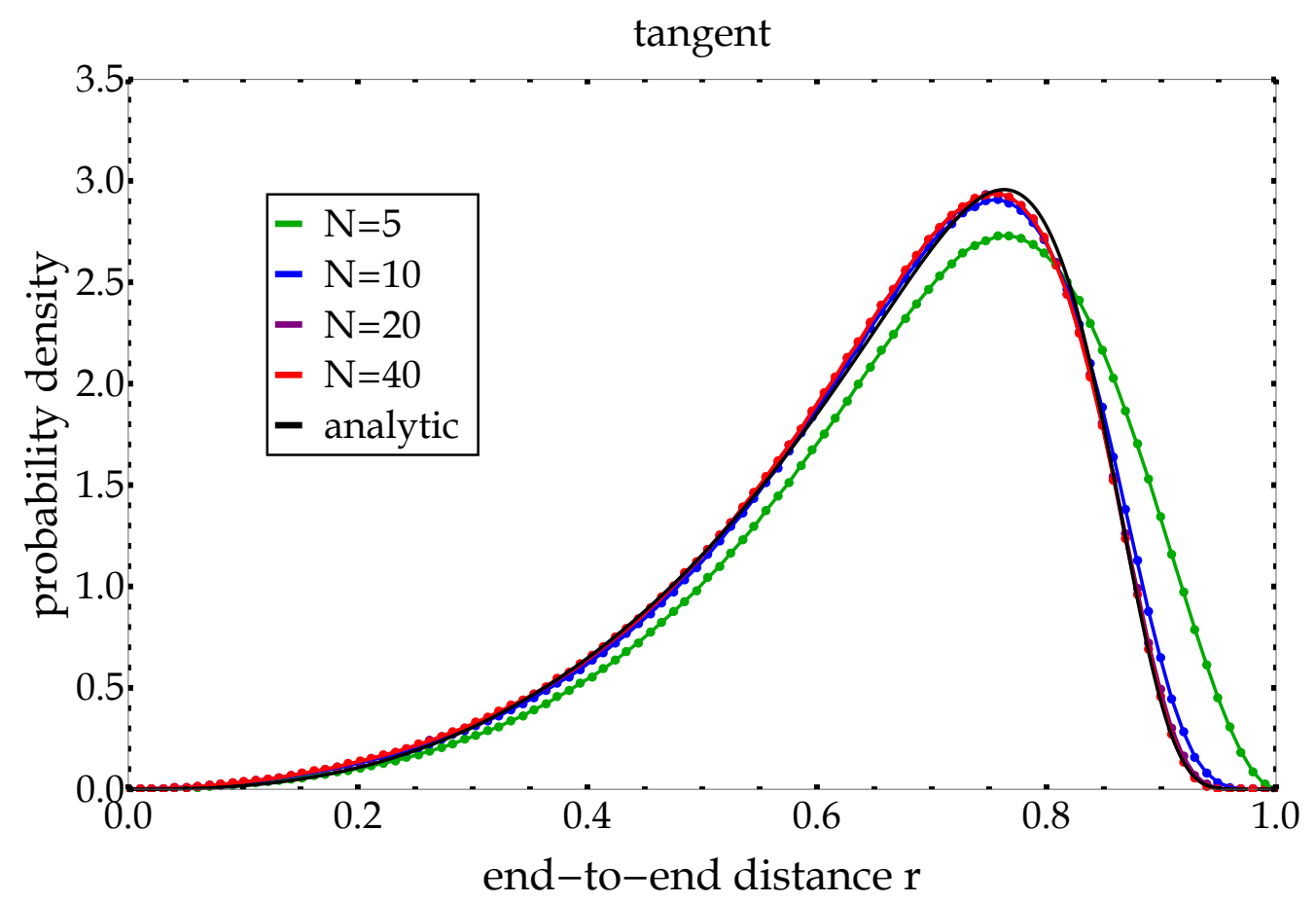

Figure A.1: The calculated end-to-end probability distribution for the tangent-based curvature for chains consisting of 5, 10, 20 and 40 vertices. The black curve shows an analytical approximation of the continuous worm-like chain limit. 


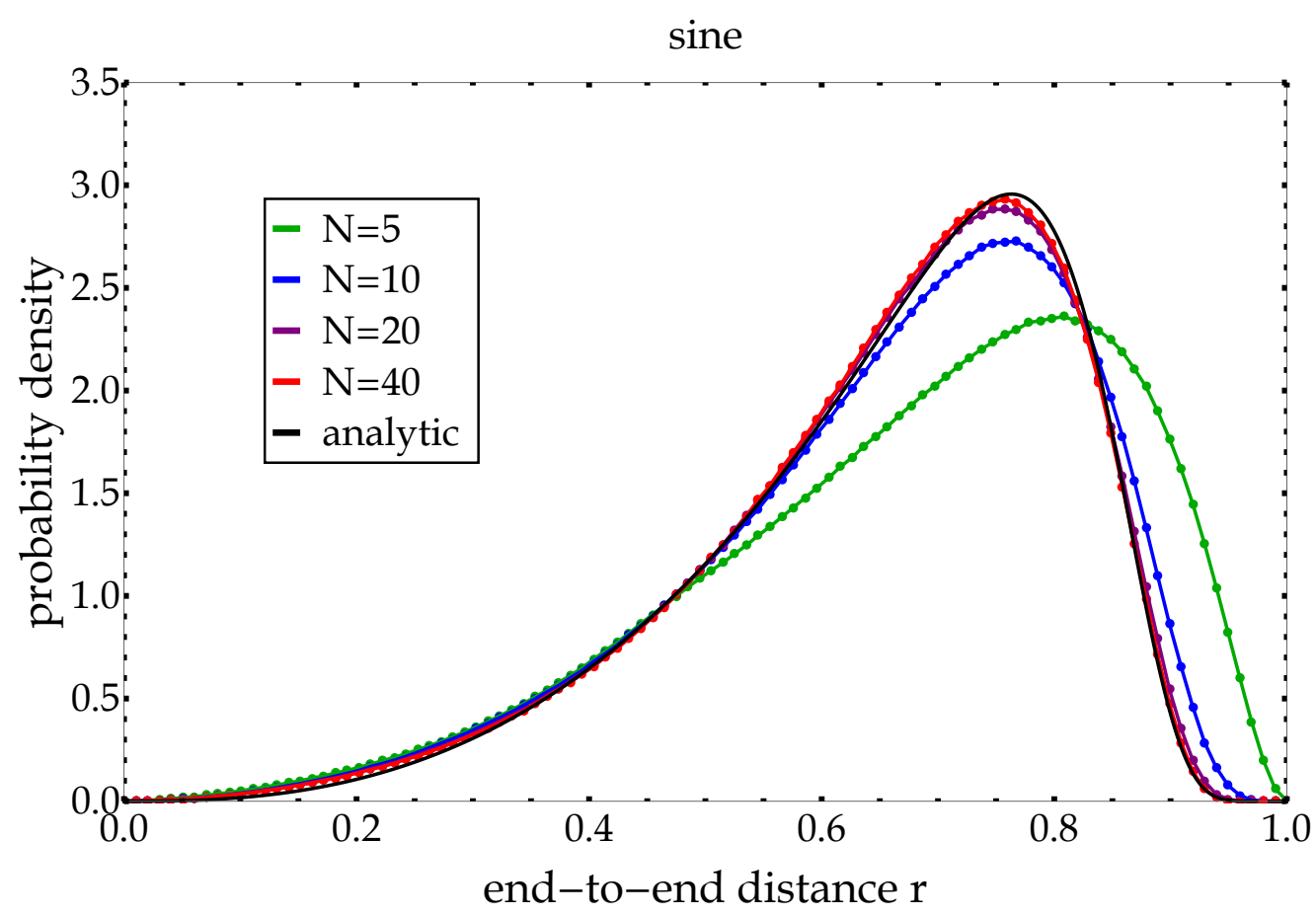

Figure A.2: The calculated end-to-end probability distribution for the sine-based curvature for chains consisting of 5,10,20 and 40 vertices. The black curve shows an analytical approximation of the continuous worm-like chain limit. 


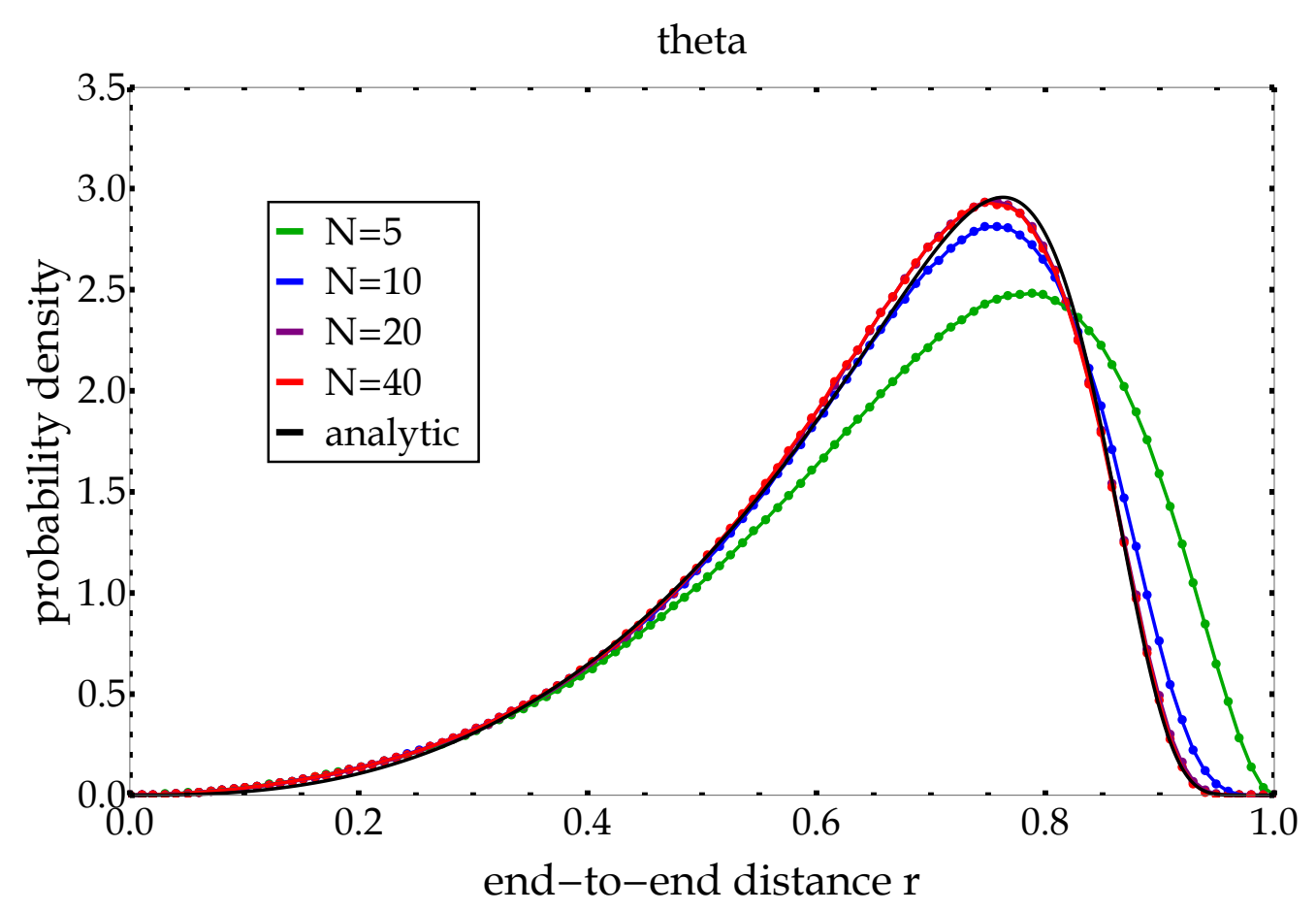

Figure A.3: The calculated end-to-end probability distribution for the $\theta$-based curvature for chains consisting of 5,10,20 and 40 vertices. The black curve shows an analytical approximation of the continuous worm-like chain limit. 


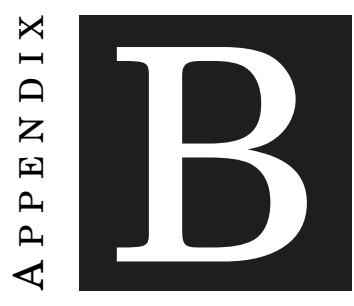

\section{PAWULA'S THEOREM}

Proofs of Pawula's theorem, similar to the one presented here, can be found in references [97] and [104]. The context of this appendix is explained in section 2.2.3 on page 36. There, we found that the jump moments $\alpha_{k}(x)$ uniquely define a Markov process from the Kramers-Moyal expansion (in orders of $k$ ) of its Master equation. Now we proof that the Kramer-Moyal expansion either ends after one term, two terms, or it never ends. This fact is known as Pawula's theorem [104]. The generalized Schwartz inequality reads

$$
\left(\int f(z) g(z) \rho(z) \mathrm{d} z\right)^{2} \leq\left(\int f^{2}(z) \rho(z) \mathrm{d} z\right) \cdot\left(\int g^{2}(z) \rho(z) \mathrm{d} z\right)
$$

where $\rho(z)$ is some nonnegative function and $f(x)$ and $g(x)$ are arbitrary functions. In the context of section 2.2.3, we choose $\rho(z)=p(z, t+\tau \mid x, t)$ which is by definition nonnegative, $f=(z-x)^{n}$ and $g(z)=(z-x)^{n+m}$ for an arbitrary, fixed $x$ and $n, m \in \mathbb{N}_{0}$. Inserting these choices into the Schwartz inequality yields

$$
\left(\Psi^{(2 n+m)}\right)^{2} \leq \Psi^{(2 n)} \cdot \Psi^{(2 n+2 m)}
$$

where

$$
\begin{aligned}
\Psi^{(k)} & =\int(z-x)^{n} p(z, t+\tau \mid x, t) \mathrm{d} z \\
& =\alpha^{(k)} \tau+\mathscr{O}\left(\tau^{2}\right)
\end{aligned}
$$

As we found in the main text, this relation between $\Psi^{(k)}$ and $\alpha^{(k)}$ only holds for $k \geq 1$. For $n=m=0$, as well as for $m=0$ while $n \geq 1$, the inequality above is trivially fulfilled. 
For $n=0$ and $m \geq 1$ we have

$$
\begin{aligned}
\left(\Psi^{(m)}\right)^{2} & \leq 1 \cdot \Psi^{(2 m)} \\
\Leftrightarrow \quad\left[\alpha^{(m)} \tau+\mathscr{O}\left(\tau^{2}\right)\right]^{2} & \leq \alpha^{(2 m)} \tau+\mathscr{O}\left(\tau^{2}\right) \quad \mid: \tau, \quad \lim _{\tau \rightarrow 0} \\
0 & \leq \alpha^{(2 m)}
\end{aligned}
$$

which is interesting but also clear from the definition of the jump moments. In conclusion, we only need to consider $n, m \geq 1$, for which the last line of equation B.3 holds true. Inserting it into the inequality, then dividing by $\tau^{2}$ and considering the limit $\tau \rightarrow 0$, we arrive at

$$
\left(\alpha^{(2 n+m)}\right)^{2} \leq \alpha^{(2 n)} \cdot \alpha^{(2 n+2 m)} \quad \text { for } \quad n, m \geq 1 \quad \text {. }
$$

From this inequality follow two conclusions:

$$
\begin{aligned}
\alpha^{(2 n)} & =0 & \Rightarrow & \alpha^{(2 n+m)}=0 \\
\text { and } \quad \alpha^{(2 n+2 m)} & =0 & \Rightarrow & \alpha^{(2 n+m)}=0 \quad .
\end{aligned}
$$

This means that if one finds some even moment $\alpha^{(2 r)}(x)=0, r \in \mathscr{N}$, all higher moments also vanish (first line).

Furthermore, since we may choose any combination $(n, m) \in\{(1, r-1),(2, r-2), \ldots,(r-1,1)\}$ in the second line such that $n+m=r$, we learn that $\alpha_{r+1}(x), \alpha_{r+2}(x) \ldots, \alpha_{2 r-1}(x)$ also vanish. Among these latter moments, there might then be more even moments which we now know to equal zero as well, and hence the whole argument can be repeated. The reader is highly encouraged to e.g. assume $\alpha_{8}=0$ and see what follows. It turns out that only three scenarios are possible: The Kramers-Moyal expansion (ii) ends after the first term (deterministic dynamics of the Liouville equation), (ii) ends after the second term (diffusive dynamics) or (iii) has an infinite number of nonzero terms including all even moments (discontinuous jump processes). 


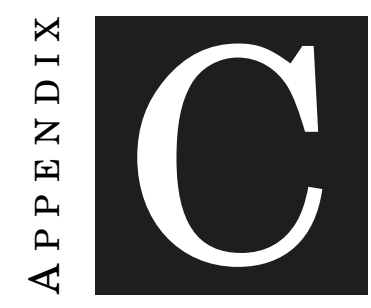

\section{ENTROPIC PSEUDOFORCE}

The physical context of this appendix is explained in section 2.2.6 on page 48. Here, we consider the statistical properties of a stochastic process whose dynamics is governed by an unconstrained Langevin equation in $3 N$ dimensions which reads

$$
\mathrm{d}_{t} \vec{r}=T^{-1} \mathbf{D} \cdot[-\vec{\nabla} E]+\sqrt{2} \mathbf{B} \cdot \vec{\xi}
$$

The Boltzmann distribution $p \propto \exp (-E / T)$ is sampled as long as the relation $\mathbf{D}=\mathbf{B} \cdot \mathbf{B}^{T}$ is fulfilled. For convenience, we choose the unity matrix $\mathbf{D}=T \mathbf{1}_{3 N \times 3 N}$ (absorbing the physical dimension into the time scale). The dynamics shall closely approximate the constraints

$$
g_{k}(\vec{r})=0 \quad \text { where } \quad k=1, \ldots, M
$$

due to the presence of strong restoring forces $-\vec{\nabla} E$ acting on the system. Fluctuations around the minimum of $E$ are very small such that it becomes a quadratic function of the (infinitesimal) deviations $\delta g_{k}$ from the constraints. The resulting Langevin equation then reads

$$
\mathrm{d}_{t} \vec{r}=-\alpha \sum_{j=1}^{M} \delta g_{j} \vec{\nabla} g_{j}+\ldots
$$

where the abbreviated terms remain constant as the elastic constant $\alpha \rightarrow \infty$. According to the chain rule, the dynamics of each of the $k=1, \ldots, M$ deviations satisfies

$$
\begin{aligned}
\mathrm{d}_{t} \delta g_{k} & =\vec{\nabla} g_{k} \cdot \mathrm{d}_{t} \vec{r} \\
& =\vec{\nabla} g_{k} \cdot\left(-\alpha \sum_{j=1}^{M} \delta g_{j} \vec{\nabla} g_{j}+\ldots\right) \\
& =-\alpha \sum_{j=1}^{M} \vec{\nabla} g_{k} \cdot \vec{\nabla} g_{j} \cdot \delta g_{j}+\ldots
\end{aligned}
$$


Rewriting this set of equations in vector notation $\delta \vec{g}=\left(\delta g_{1}, \ldots, \delta g_{M}\right)$ yields

$$
\mathrm{d}_{t} \delta \vec{g}=-\alpha \mathbf{G} \cdot \delta \vec{g}+\ldots
$$

$$
\text { where } G_{k j}=\partial_{i} g_{k} \cdot \partial_{i} g_{j} \quad \text {. }
$$

Next we transform this equation into the eigensystem of matrix $\mathbf{G}$ by multiplying both sides (from the left) with the orthonormal matrix $\mathbf{S}=\left(\mathbf{S}^{T}\right)^{-1}$ containing its eigenvectors:

$$
\begin{aligned}
\mathrm{d}_{t} \vec{v} & =-\alpha \hat{\mathbf{G}} \cdot \vec{v}+\ldots \\
\text { where } \quad \vec{v} & =\mathbf{S} \cdot \delta \vec{g} \\
\text { and } \quad \hat{\mathbf{G}} & =\mathbf{S} \cdot \mathbf{G} \cdot \mathbf{S}^{T}
\end{aligned}
$$

Since $\hat{\mathbf{G}}$ is diagonal, the dynamics of the components of $\vec{v}$ occur independently, all experiencing harmonic potentials

$$
E_{k}=\frac{\alpha}{2} \lambda_{k} v_{k}^{2}
$$

where the $\lambda_{k}$ are the eigenvalues (diagonal entries) of $\hat{\mathbf{G}}$. The probability (Boltzmann) distribution for $v_{k}$ is hence a Gaussian with zero mean and variance

$$
\sigma_{k}^{2} \propto \frac{1}{\lambda_{k}}
$$

The entropy of a Gaussian with variance $\sigma^{2}$ equals $\frac{1}{2} \ln \left(2 \pi e \sigma^{2}\right)$, and therefore the entropy $S_{k}$ of the fluctuations of $v_{k}$ are

$$
S_{k}=-\frac{1}{2} \ln \left(\lambda_{k}\right)+\text { const. }
$$

It follows that the total entropy $S_{C}$ of the fluctuations of the vector $\vec{v}$ is

$$
\begin{aligned}
S_{C} & =\sum_{k} S_{k} \\
& =-\frac{1}{2} \sum_{k} \ln \left(\lambda_{k}\right)+\text { const. } \\
& =-\frac{1}{2} \ln \left(\prod_{k} \lambda_{k}\right)+\text { const. } \\
& =-\frac{1}{2} \ln (\operatorname{det} \hat{\mathbf{G}})+\text { const. } \\
& =-\frac{1}{2} \ln (\operatorname{det} \mathbf{G})+\text { const. }
\end{aligned}
$$

For the constrained Langevin equation (main text) we only require the gradient of this entropy with respect to the Cartesian coordinates $\vec{r}$. For this reason the neglected terms (including $\alpha$ and $T$ ) in the calculation above are indeed inconsequential as they vanish upon differentiation. 
The matrix $\mathbf{G}$ does depend on $\vec{r}$, however, because the constraints $g_{k}$ themselves do, and therefore the force term

$$
\vec{\nabla} S_{C}=-\frac{1}{2} \vec{\nabla} \ln \operatorname{det} \mathbf{G}
$$

is all we need to report back to the main text.

Inextensibility constraints The constraint entropy's gradient can be evaluated for specific constraints. In this thesis in particular, we consider inextensibility constraints for a discrete chain consisting of $N$ beads:

$$
\begin{aligned}
g_{k} & =\left|\vec{r}_{k+1}-\vec{r}_{k}\right|-1=0 \\
\text { such that } \quad \vec{\nabla}_{i} g_{k}(\vec{x}) & =\frac{\vec{r}_{k+1}-\vec{r}_{k}}{\left|\vec{r}_{k+1}-\vec{r}_{k}\right|} \cdot\left(\delta_{i+1, k}-\delta_{i, k}\right)=\vec{t}_{k} \cdot\left(\delta_{i+1, k}-\delta_{i, k}\right)
\end{aligned}
$$

where $k=1, \ldots, N-1$. The matrix elements of $\mathbf{G}$ then read

$$
G_{k j}=\sum_{i=1}^{N} \vec{\nabla}_{i} g_{k} \cdot \vec{\nabla}_{i} g_{j}=\sum_{i=1}^{N} \vec{t}_{k} \cdot\left(\delta_{i+1, k}-\delta_{i, k}\right) \cdot \vec{t}_{j} \cdot\left(\delta_{i+1, j}-\delta_{i, j}\right)= \begin{cases}-\vec{t}_{k} \cdot \vec{t}_{j} & \text { if }|j-k|=1 \\ 2 & \text { if } j=k \\ 0 & \text { otherwise }\end{cases}
$$

Making use of the fact that only the gradients of the next diagonals of $\mathbf{G}$ are nonzero, the gradient of $\ln \operatorname{det} \mathbf{G}$ can be calculated using the following identity [66]:

$$
\vec{\nabla}_{i} \ln \operatorname{det} \mathbf{G}=\sum_{j, k=1}^{M} \frac{1}{\operatorname{det} \mathbf{G}} \cdot \frac{\partial \operatorname{det} \mathbf{G}}{\partial G_{j k}} \cdot \vec{\nabla}_{i} G_{j k}=\sum_{j, k=1}^{M}\left(G^{-1}\right)_{j k} \cdot \vec{\nabla}_{i} G_{j k}=\sum_{j=1}^{M-1}\left(G^{-1}\right)_{j, j+1} \cdot \vec{\nabla}_{i} G_{j, j+1}
$$

The last factor can easily be evaluated analytically, and the first factor (of the final sum) requires knowledge of the next diagonal elements of the inverse matrix $\mathbf{G}^{-1}$, whose numerical evaluation is straightforward (but unfortunately depends on the Cartesian coordinates $\vec{r}$ ).

Bound-end constraint Binding one end of a chain to a fixed point in space (the origin) can be achieved by implementing the constraint

$$
g_{0}(\vec{r})=\left|\vec{r}_{1}\right|-1=0 \quad \text {. }
$$

For a chain consisting of $N$ beads, this additional constraint turns the $N-1 \times N-1$-dimensional matrix $\mathbf{G}$ into an $N \times N$-dimensional matrix. Apart from its size, it is almost identical to the matrix for the inextensibility constraints alone. The only difference is that its first element is $G_{1,1}=1$, and the first "tangent vector" must be taken to be $\vec{r}_{1} /\left|\vec{r}_{1}\right|$ (assuming the chain end is bound to the origin of our coordinate system). 



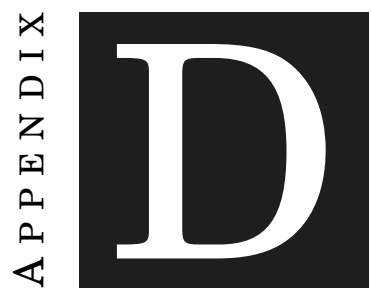

\section{ANGULAR CORRELATIONS IN WORM-LIKE}

CHAINS

The context of this appendix is explained in section 2.4 on page 66 . The following calculation can also be found in the textbook by Doi and Edwards [115].

For a discrete WLC with a bending energy present, the average cosine of a bending angle $\theta$ can be directly calculated from the Boltzmann distribution via

$$
\langle\cos \theta\rangle=\frac{\int_{-1}^{1} y \cdot \exp \left(-\frac{\sigma}{2 d}\left(2 \tan \left(\frac{\arccos y}{2}\right)\right)^{2}\right) \mathrm{d} y}{\int_{-1}^{1} \exp \left(-\frac{\sigma}{2 d}\left(2 \tan \left(\frac{\arccos y}{2}\right)\right)^{2}\right) \mathrm{d} y}
$$

where $y:=\cos \theta$. Let us now consider the direction of a tangent vector $\vec{t}_{i}$. Since no energy is associated with rotations of $\vec{t}_{i}$ around $\vec{t}_{i-1}$, all such rotational (torsion) angles are equally probable and we have

$$
\left\langle\vec{t}_{i}\right\rangle=\langle\cos \theta\rangle \vec{t}_{i-1}
$$

Multiplying both sides with $\vec{t}_{k}$, and then averaging over its orientations yields

$$
\left\langle\vec{t}_{i} \cdot \vec{t}_{k}\right\rangle=\langle\cos \theta\rangle\left\langle\vec{t}_{i-1} \cdot \vec{t}_{k}\right\rangle
$$

This is a recursion rule for the vectorial correlations $\left\langle\vec{t}_{i} \cdot \vec{t}_{k}\right\rangle$ where $k$ is fixed and $i$ is the variable index. 
Together with the initial condition $\left\langle\vec{t}_{k}^{2}\right\rangle=1$, the recursion rule is solved by

(D.4)

$$
\left\langle\vec{t}_{i} \cdot \vec{t}_{k}\right\rangle=\langle\cos \theta\rangle^{|i-k|},
$$

which is an exponential decay along the chain. Defining the persistence length $l_{P}=-\frac{d}{\ln \langle\cos \theta\rangle}$, we may alternatively write

$$
\left\langle\vec{t}_{1} \cdot \vec{t}_{k+1}\right\rangle=\exp \left(-\frac{k d}{l_{P}}\right)
$$

where $d$ is the bond length of the discrete WLC.

The analogous behavior of the continuous WLC immediately follows from this result by considering the limit $d \rightarrow 0$ while holding $l_{P}$ fixed, in which case $k \cdot d$ turns into the continuous variable $s$ called arc length. 


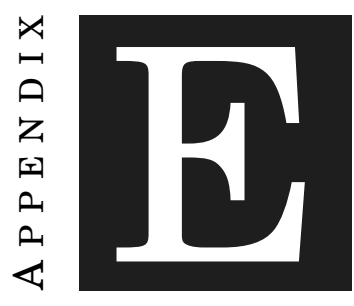

\section{Solving CONSTRAINTS}

The context of this appendix is explained in section 4.2 on page 87 . Here, we merely insert the equations of motion

$$
\mathrm{d} \vec{r}_{i}=\sum_{j=1}^{N} \mathbf{D}_{i j} \cdot\left[\vec{F}_{j}^{\mathrm{uc}}+\lambda_{j} \vec{t}_{j}-\lambda_{j-1} \vec{t}_{j-1}\right] \mathrm{d} t
$$

where

$$
\vec{F}^{\mathrm{uc}}=-\vec{\nabla}\left(E-S_{C}\right)+\mathbf{D}^{-1} \cdot\left(\vec{\nabla}^{T} \cdot \mathbf{D}\right)+\sqrt{2}\left(\mathbf{B}^{T}\right)^{-1} \mathrm{~d} \vec{W} / \mathrm{d} t
$$

into the constraints

$$
\begin{aligned}
& 0 \stackrel{!}{=} \vec{t}_{0}^{T} \cdot \mathrm{d} \vec{r}_{1} \\
& 0 \stackrel{!}{=} \vec{t}_{\mu}^{T} \cdot\left(\mathrm{d} \vec{r}_{\mu+1}-\mathrm{d} \vec{r}_{\mu+1}\right), \quad \mu=1, \ldots, N-1,
\end{aligned}
$$

with the goal of rewriting them in the simple form

$$
\sum_{v=0}^{N-1} M_{\mu v} \lambda_{v}=v_{\mu} \quad \mu=0, \ldots, N-1 \quad .
$$

Before we start, let us define the following $3 \times 3$-matrices:

$$
\begin{aligned}
\mathbf{G}_{\mu v}:=\mathbf{D}_{\mu v}-\mathbf{D}_{\mu, v+1}, & \mu=1, \ldots, N, & v=1, \ldots, N-1 \\
\mathbf{H}_{\mu v}:=\mathbf{D}_{\mu+1, v}-\mathbf{D}_{\mu v}, & \mu=1, \ldots, N-1, & v=1, \ldots, N \\
\mathbf{C}_{\mu v}:=\mathbf{H}_{\mu v}-\mathbf{H}_{\mu, v+1}, & \mu=1, \ldots, N-1, & v=1, \ldots, N-1
\end{aligned}
$$

and remark that $\mu$ counts the constraints and $v$ counts the corresponding Lagrangian multipliers $\lambda$. 
The first constraint (bound-end, $\mu=0$ ) reads

$$
\begin{aligned}
0 & \stackrel{!}{=} \vec{t}_{0}^{T} \cdot \mathrm{d} \vec{r}_{1} \\
& =\sum_{j=1}^{N} \vec{t}_{0}^{T} \cdot \mathbf{D}_{1 j} \cdot\left[\vec{F}_{j}^{\mathrm{uc}}+\lambda_{j} \vec{t}_{j}-\lambda_{j-1} \vec{t}_{j-1}\right] \\
& =\sum_{j=1}^{N} \vec{t}_{0}^{T} \cdot \mathbf{D}_{1 j} \cdot \vec{F}_{j}^{\mathrm{uc}}+\sum_{j=1}^{N-1} \vec{t}_{0}^{T} \cdot \mathbf{D}_{1 j} \cdot \lambda_{j} \vec{t}_{j}-\sum_{j=1}^{N} \vec{t}_{0}^{T} \cdot \mathbf{D}_{1 j} \cdot \lambda_{j-1} \vec{t}_{j-1} \\
& =\sum_{j=1}^{N} \vec{t}_{0}^{T} \cdot \mathbf{D}_{1 j} \cdot \vec{F}_{j}^{\mathrm{uc}}+\sum_{j=1}^{N-1} \vec{t}_{0}^{T} \cdot \mathbf{D}_{1 j} \cdot \lambda_{j} \vec{t}_{j}-\sum_{j=0}^{N-1} \vec{t}_{0}^{T} \cdot \mathbf{D}_{1, j+1} \cdot \lambda_{j} \vec{t}_{j} \\
& =\sum_{j=1}^{N} \vec{t}_{0}^{T} \cdot \mathbf{D}_{1 j} \cdot \vec{F}_{j}^{\mathrm{uc}}+\sum_{j=1}^{N-1} \vec{t}_{0}^{T} \cdot\left(\mathbf{D}_{1 j}-\mathbf{D}_{1, j+1}\right) \cdot \lambda_{j} \vec{t}_{j}-\vec{t}_{0}^{T} \cdot \mathbf{D}_{11} \cdot \lambda_{0} \vec{t}_{0} \\
& =\sum_{j=1}^{N} \vec{t}_{0}^{T} \cdot \mathbf{D}_{1 j} \cdot \vec{F}_{j}^{\mathrm{uc}}+\sum_{j=1}^{N-1}\left(\vec{t}_{0}^{T} \cdot \mathbf{G}_{1 j} \cdot \vec{t}_{j}\right) \lambda_{j}-\left(\vec{t}_{0}^{T} \cdot \mathbf{D}_{11} \cdot \vec{t}_{0}\right) \lambda_{0}
\end{aligned}
$$

and the remaining $N-1$ of the constraints (inextensibility, $\mu=1, \ldots, N-1$ ) are

(E.7)

$$
\begin{aligned}
0 & \stackrel{!}{=} \vec{t}_{\mu}^{T} \cdot\left(\mathrm{d} \vec{r}_{\mu+1}-\mathrm{d} \vec{r}_{\mu+1}\right) \\
& =\vec{t}_{\mu}^{T} \cdot \sum_{j=1}^{N}\left(\mathbf{D}_{\mu+1, j}-\mathbf{D}_{\mu j}\right) \cdot\left[\vec{F}_{j}^{\mathrm{uc}}+\lambda_{j} \vec{t}_{j}-\lambda_{j-1} \vec{t}_{j-1}\right] \\
& =\sum_{j=1}^{N} \vec{t}_{\mu}^{T} \cdot \mathbf{H}_{\mu j} \cdot\left[\vec{F}_{j}^{\mathrm{uc}}+\lambda_{j} \vec{t}_{j}-\lambda_{j-1} \vec{t}_{j-1}\right] \\
& =\sum_{j=1}^{N} \vec{t}_{\mu}^{T} \cdot \mathbf{H}_{\mu j} \cdot \vec{F}_{j}^{\mathrm{uc}}+\sum_{j=1}^{N-1} \vec{t}_{\mu}^{T} \cdot \mathbf{H}_{\mu j} \cdot \lambda_{j} \vec{t}_{j}-\sum_{j=1}^{N} \vec{t}_{\mu}^{T} \cdot \mathbf{H}_{\mu j} \cdot \lambda_{j-1} \vec{t}_{j-1} \\
& =\sum_{j=1}^{N} \vec{t}_{\mu}^{T} \cdot \mathbf{H}_{\mu j} \cdot \vec{F}_{j}^{\mathrm{uc}}+\sum_{j=1}^{N-1} \vec{t}_{\mu}^{T} \cdot \mathbf{H}_{\mu j} \cdot \lambda_{j} \vec{t}_{j}-\sum_{j=0}^{N-1} \vec{t}_{\mu}^{T} \cdot \mathbf{H}_{\mu, j+1} \cdot \lambda_{j} \vec{t}_{j} \\
& =\sum_{j=1}^{N} \vec{t}_{\mu}^{T} \cdot \mathbf{H}_{\mu j} \cdot \vec{F}_{j}^{\mathrm{uc}}+\sum_{j=1}^{N-1} \vec{t}_{\mu}^{T} \cdot\left(\mathbf{H}_{\mu j}-\mathbf{H}_{\mu, j+1}\right) \cdot \lambda_{j} \vec{t}_{j}-\vec{t}_{\mu}^{T} \cdot \mathbf{H}_{\mu, 1} \cdot \lambda_{0} \vec{t}_{0} \\
& =\sum_{j=1}^{N} \vec{t}_{\mu}^{T} \cdot \mathbf{H}_{\mu j} \cdot \vec{F}_{j}^{\mathrm{uc}}+\sum_{j=1}^{N-1}\left(\vec{t}_{\mu}^{T} \cdot \mathbf{C}_{\mu j} \cdot \vec{t}_{j}\right) \lambda_{j}-\left(\vec{t}_{\mu}^{T} \cdot \mathbf{H}_{\mu, 1} \cdot \vec{t}_{0}\right) \lambda_{0} .
\end{aligned}
$$

Hence we can read off that

$$
\begin{aligned}
& v_{0}=-\sum_{j=1}^{N} \vec{t}_{0}^{T} \cdot \mathbf{D}_{1 j} \cdot \vec{F}_{j}^{\mathrm{uc},}, \\
& v_{\mu}=-\sum_{j=1}^{N} \vec{t}_{\mu}^{T} \cdot \mathbf{H}_{\mu j} \cdot \vec{F}_{j}^{\mathrm{uc},} \quad \mu=1, \ldots, N-1,
\end{aligned}
$$

$$
\begin{aligned}
& M_{00}=-\vec{t}_{0}^{T} \cdot \mathbf{D}_{11} \cdot \vec{t}_{0} \\
& M_{0 v}=\vec{t}_{0}^{T} \cdot \mathbf{G}_{1 v} \cdot \vec{t}_{v}, \quad v=1, \ldots, N-1 \\
& M_{\mu 0}=-\vec{t}_{\mu}^{T} \cdot \mathbf{H}_{\mu, 1} \cdot \vec{t}_{0}, \quad \mu=1, \ldots, N-1 \\
& \text { and } \quad M_{\mu v}=\vec{t}_{\mu}^{T} \cdot \mathbf{C}_{\mu v} \cdot \vec{t}_{v}, \quad \mu, v=1, \ldots, N-1
\end{aligned}
$$


The most general case of inextensibility as well as bound-end constraints being present is thus treated. The case of a freely swimming, inextensible chain immediately follows from the line of reasoning above - one must only set $\lambda_{0}=0$ and ignore the corresponding constraint $(\mu=0)$ such that $\mu=1, \ldots, N-1$ in equation E.4. Therefore, all entries with an index which is zero can be discarded from equation E.8 and the inextensibility constraints may be written as

$$
\sum_{v=1}^{N-1} M_{\mu v} \lambda_{v}=v_{\mu}
$$

where $\mu, v=1, \ldots, N-1$,

$$
\begin{aligned}
v_{\mu} & =-\sum_{j=1}^{N} \vec{t}_{\mu}^{T} \cdot \mathbf{H}_{\mu j} \cdot \vec{F}_{j}^{\mathrm{uc}} \\
\text { and } \quad M_{\mu \nu} & =\vec{t}_{\mu}^{T} \cdot \mathbf{C}_{\mu \nu} \cdot \vec{t}_{v}
\end{aligned}
$$





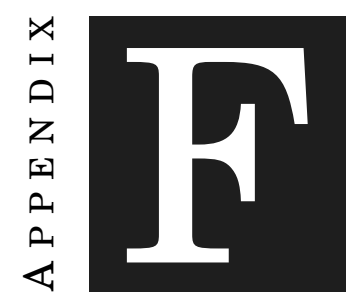

\section{DIFFUSION COEFFICIENT - BENCHMARKS}

The context of this appendix is explained in section 4.7 on page 101. Here, we show mean square displacements (MSD) as functions of time for simulated model chains. These chains have two different chain lengths; $N=5$ and $N=16$. For both chain lengths, we investigate the three cases of (i) no hydrodynamic interactions (HI) ( $a=0$ ), (ii) strong HI ( $a=1)$, and (iii) strong HI ( $a=1)$ with a fluorophore attached. The latter fluorophore mimics the real fluorophore Atto655 and has hydrodynamic radius $a_{1}=6 / 3.8$ and a fixed bond length of $d_{1}=7 / 3.8$. The bending stiffness of all chains is set to $\sigma=1$ and the excluded volume radius to $R_{\mathrm{EV}}=\sqrt{2} / 4$. For each simulation, we display the MSD of the chain's center of mass, as well as that of the first bead (which is the fluorophore if applicable).

We observe again that hydrodynamic interactions make the beads fluctuate more cooperatively. Here, this fact is reflected by the offset between the first bead's MSD and the diagonal line being smaller in case of hydrodynamic interactions. In general, the center of mass of a simulated chain also undergoes weak intrachain dynamics, as all beads are weighted equally in the center of mass, but some are more mobile than other (the free ends for instance). Hence the offset between the center of mass' MSD and the diagonal line is also allowed.

All simulated MSDs were divided by the theoretically predicted diffusion coefficient (see equation 4.22, page 96). Their slope is thus supposed to converge towards 1. "BD" stands for Brownian dynamics, i.e. simulated values, and "MC" stands for Monte-Carlo sampling, i.e. calculated values. 


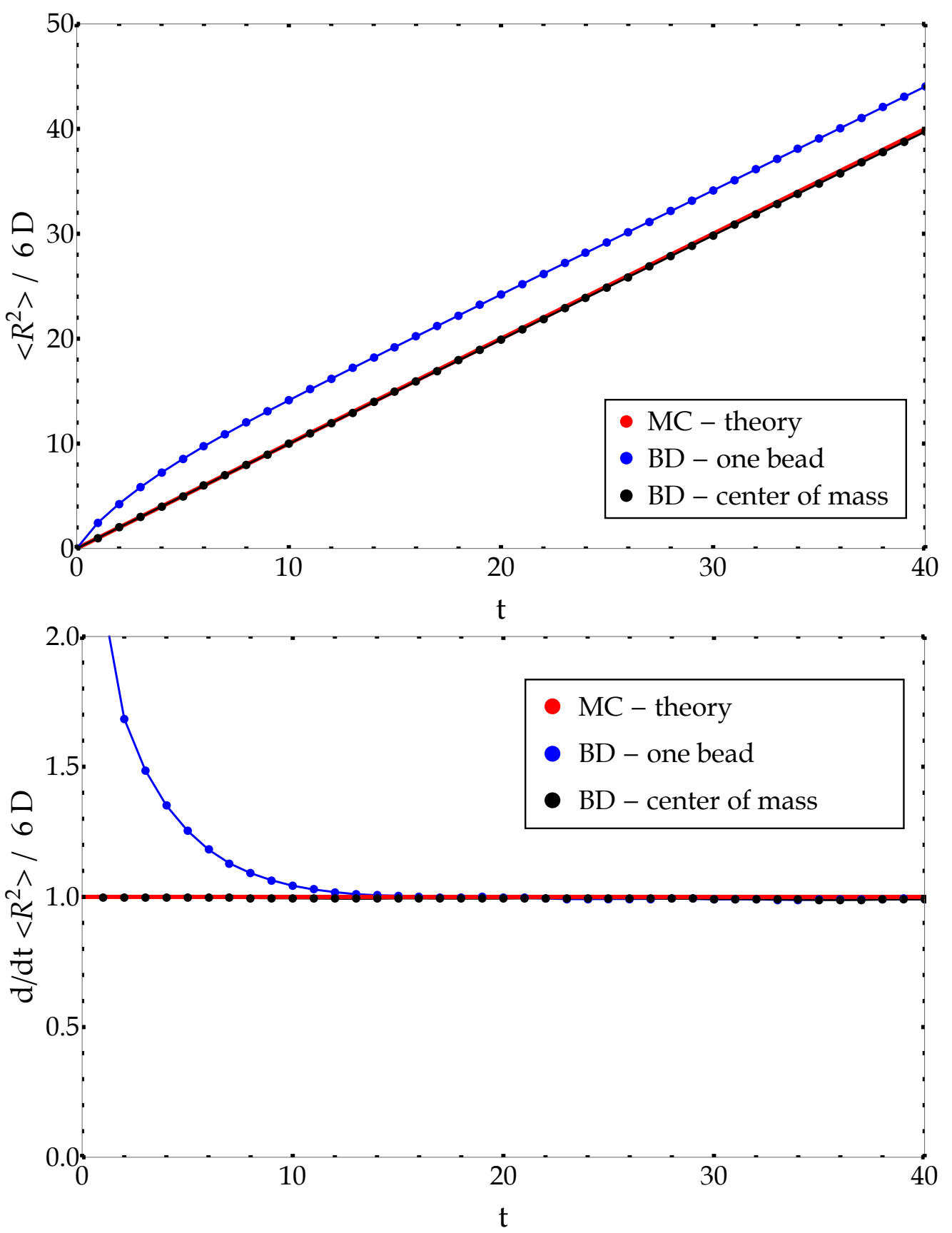

Figure F.1: $N=5, a=0$. Top: Simulated MSDs from Brownian dynamics simulations (BD) of the chain's first bead (blue) and its center of mass (black), as well as the theoretically predicted long-term behavior (red), over time. Bottom: slopes of top plot. 

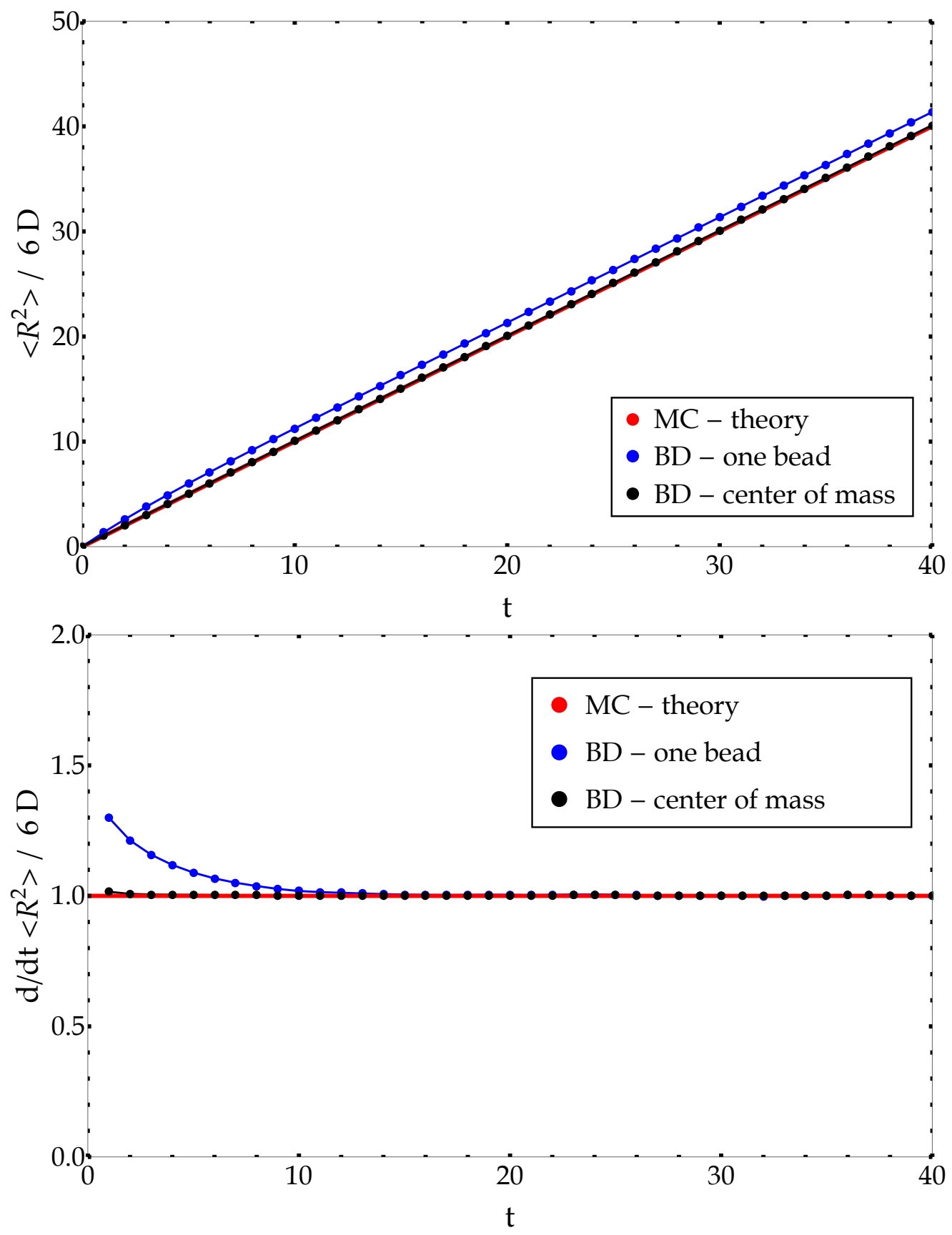

Figure F.2: $N=5, a=1$. Top: Simulated MSDs from Brownian dynamics simulations (BD) of the chain's first bead (blue) and its center of mass (black), as well as the theoretically predicted long-term behavior (red), over time. Bottom: slopes of top plot. 


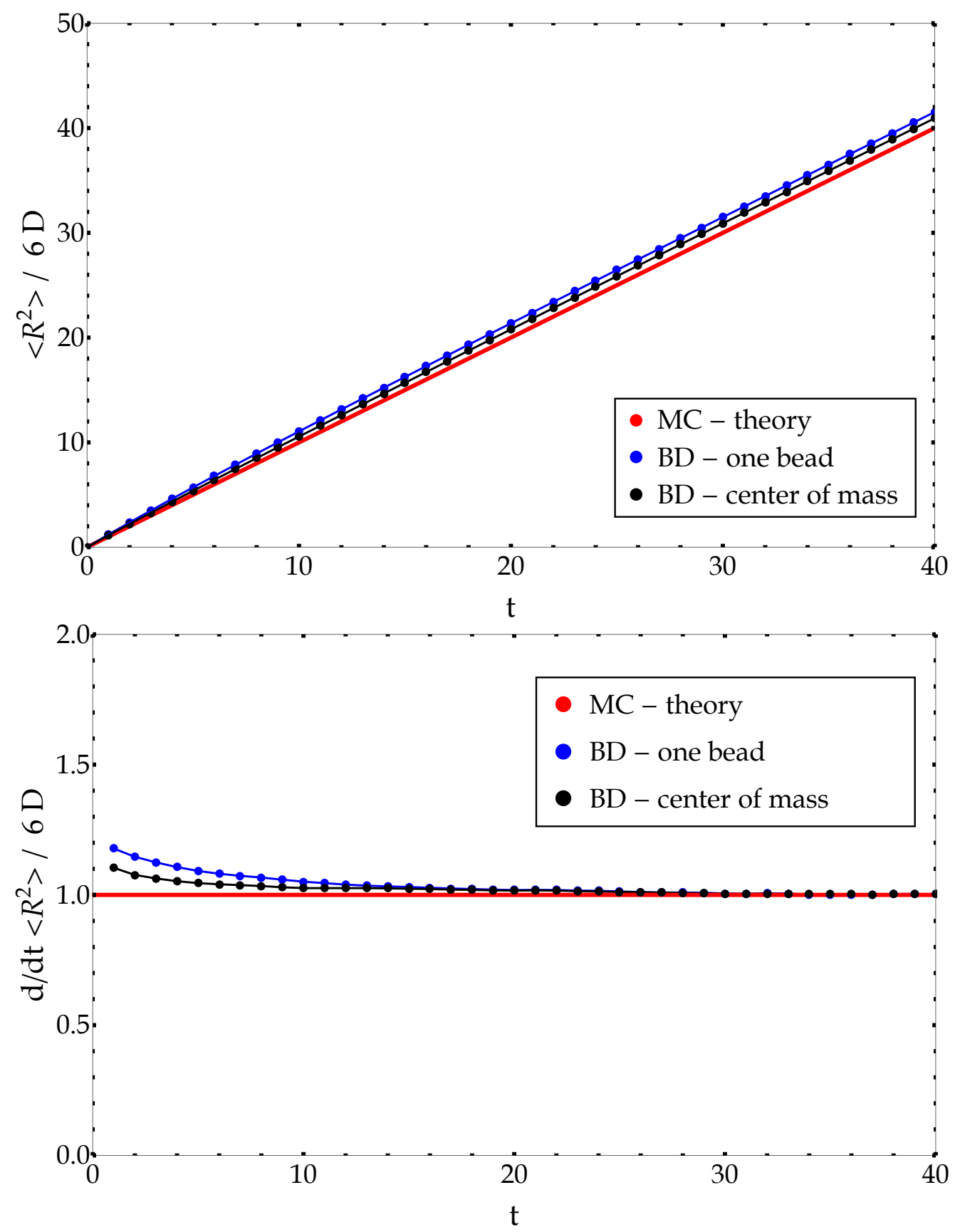

Figure F.3: $N=5, a=1$, fluorophore included. Top: Simulated MSDs from Brownian dynamics simulations (BD) of the chain's first bead (blue) and its center of mass (black), as well as the theoretically predicted long-term behavior (red), over time. Bottom: slopes of top plot. 

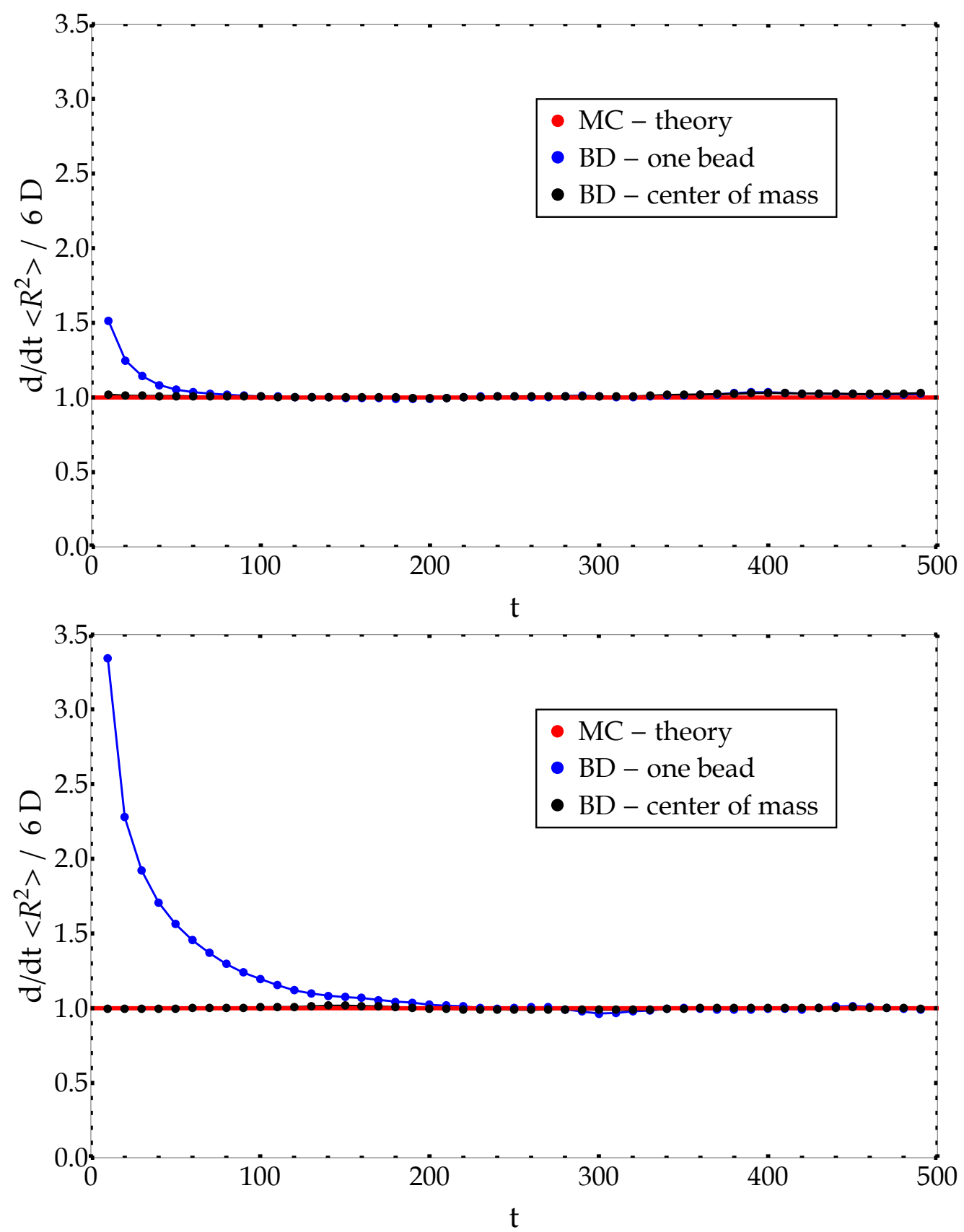

Figure F.4: $N=16, a=0$. Top: Simulated MSDs from Brownian dynamics simulations (BD) of the chain's first bead (blue) and its center of mass (black), as well as the theoretically predicted long-term behavior (red), over time. Bottom: slopes of top plot. 

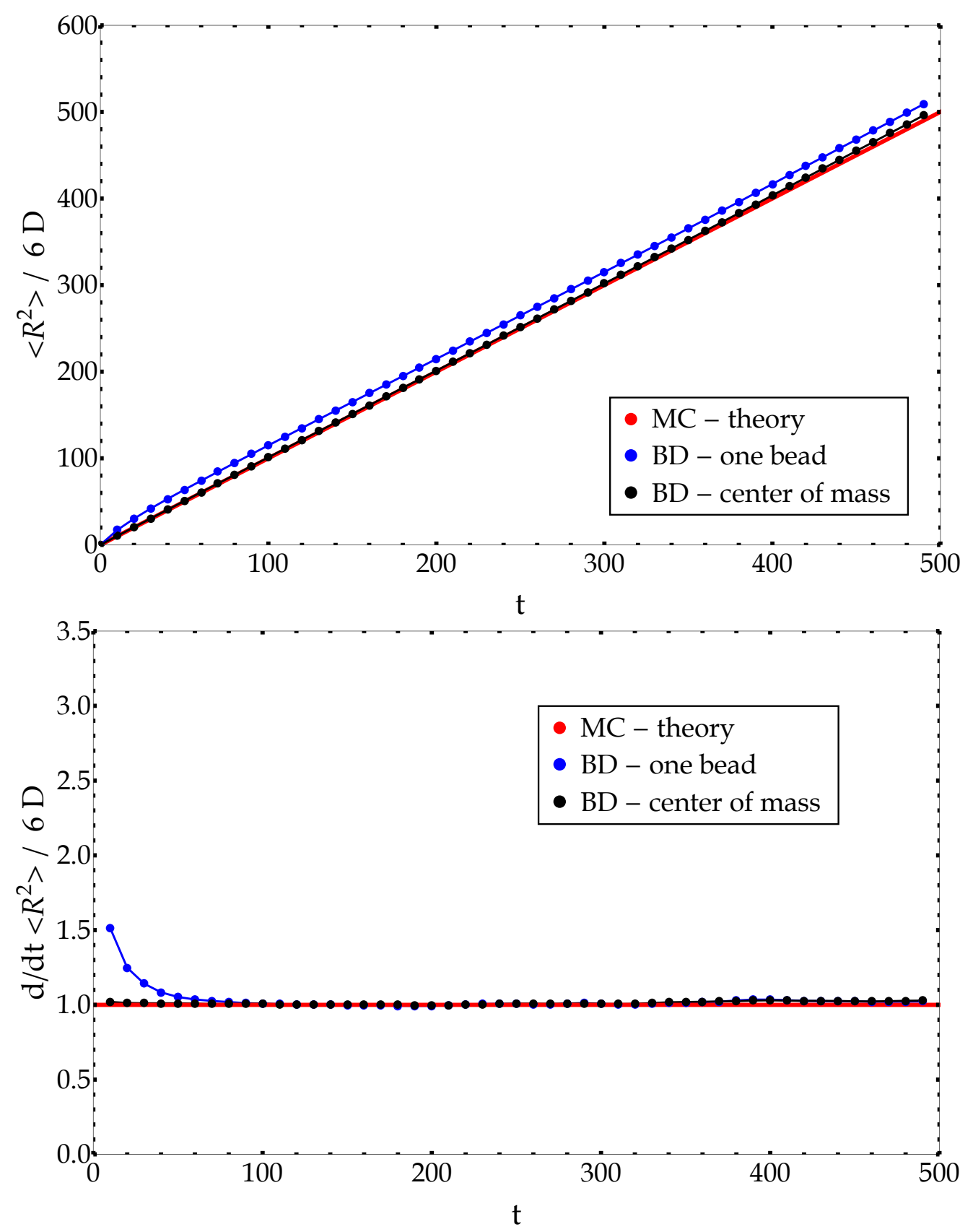

Figure F.5: $N=16, a=1$. Top: Simulated MSDs from Brownian dynamics simulations (BD) of the chain's first bead (blue) and its center of mass (black), as well as the theoretically predicted long-term behavior (red), over time. Bottom: slopes of top plot. 


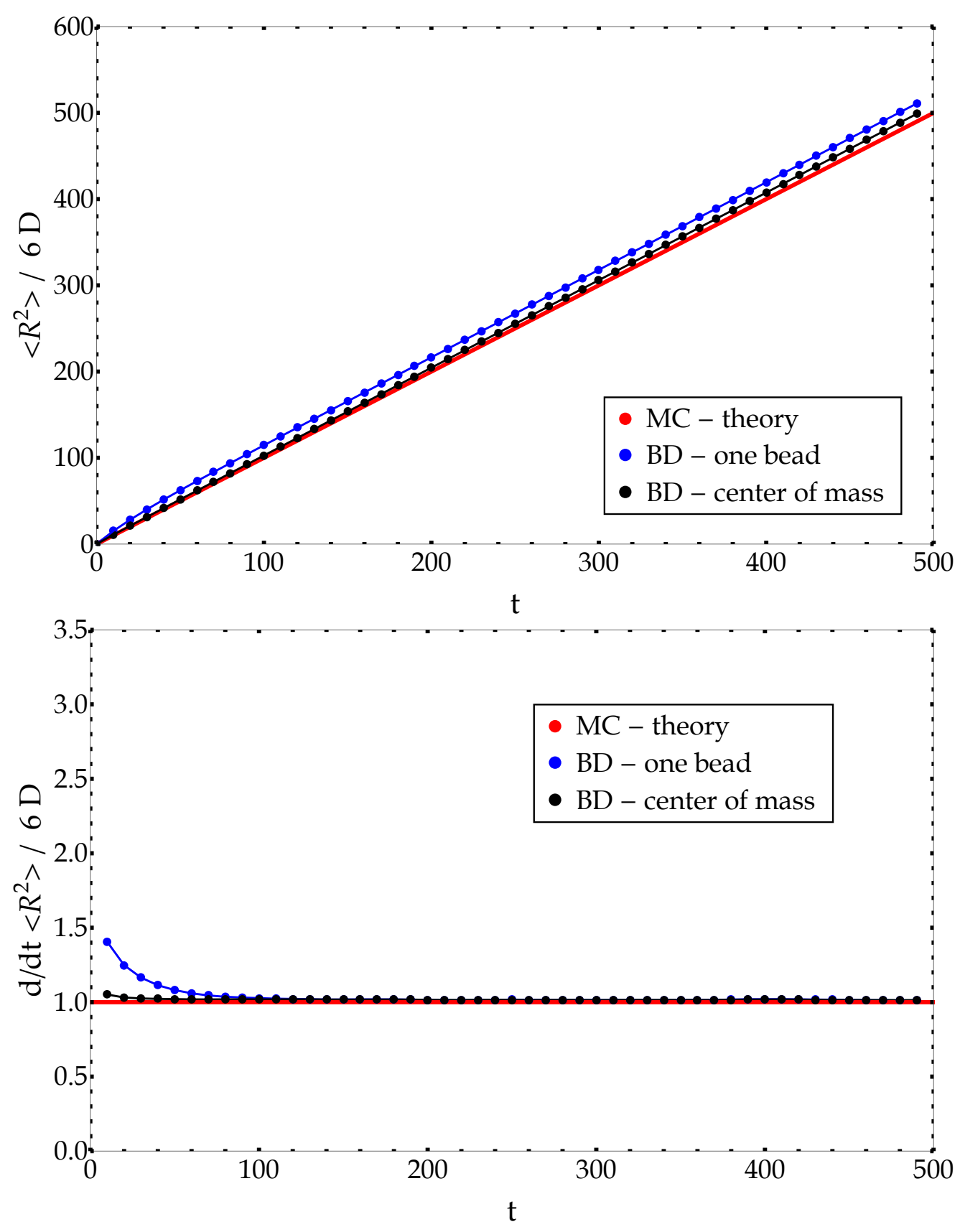

Figure F.6: $N=16, a=1$, fluorophore included. Top: Simulated MSDs from Brownian dynamics simulations (BD) of the chain's first bead (blue) and its center of mass (black), as well as the theoretically predicted long-term behavior (red), over time. Bottom: slopes of top plot. 



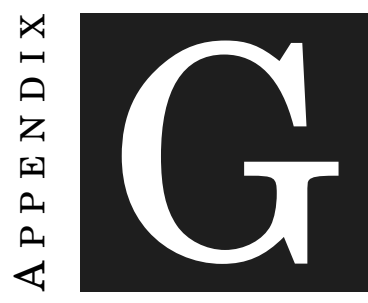

\section{SECONDARY STRUCTURES AND TWIST} ELASTICITY

In this appendix, we elaborate on extending our polymer model to take into account secondary structures and twist elasticity. The context for this is explained in section 7.4 on page 154 .

Throughout this thesis, the elastic energy only took into account bending effects in an isotropic manner. Now, we utilize the most general discrete elastic energy (as described in section 2.3.2, page 61) which reads

$$
E=\sum_{i=1}^{N-2}\left(\frac{\alpha}{2}\left(\Omega_{i}^{(1)}-\bar{\Omega}_{i}^{(1)}\right)^{2}+\frac{\alpha}{2}\left(\Omega_{i}^{(2)}-\bar{\Omega}_{i}^{(2)}\right)^{2}+\frac{c}{2}\left(\Omega_{i}^{(3)}\right)^{2}\right) \Delta s_{i}
$$

Twist elasticity Let us first investigate the effect of twist elasticity. To this end, we set the reference curvatures $\bar{\Omega}$ to zero, but do consider a finite twist elasticity $c$. The simulation algorithm for $c \neq 0$ is that described in the main text, but please note that now the material frame needs to be kept track of during the simulation. Interestingly, the influence of twist elasticity does not alter the Boltzmann distribution for the chain's vertex position. That is because the new energy term concerns only variables which can take on values independently of the chain's centerline: the twist angles. Therefore, the joined probability distribution for the vertex positions (i.e. bond angles) and twist angles factorizes. Statistically speaking, they are thus entirely independent. I have successfully checked that the simulated twist angle distribution indeed follows its Boltzmann distribution (data not shown). The twist gradient with respect to the vertex positions does not 
vanish (out-of-plane bending induces twist), however, and thus the vertex and twist dynamics are coupled. It may therefore be expected that finite twist elasticity $c$ influences dynamical observables of the chain dynamics, but not the vertices' statistical properties. For this reason, let us simulate chains with varying twist elasticity and study its influence on (i) the end-to-end contact rate and (ii) the end-to-end reconfiguration time (time scale on which its autocorrelation decays mono-exponentially). We simulated chains with parameters $N=10, \sigma=1, a=0$ and $c \in\{1,3,5,7\}$, where $c$ is measured in units of $T \cdot d$ in the same way that $\sigma$ is. The result is shown in figure G.1. We observe that both the reconfiguration and the loop formation time scale increase with $c$. This increase is relatively weak, however, considering that a value of $c=7$ makes a twist angle of one exp (7) times less probable than a twist angle of zero! We must conclude that twist elasticity would be very difficult to detect experimentally using PET-FCS or dynaMIET experiments.

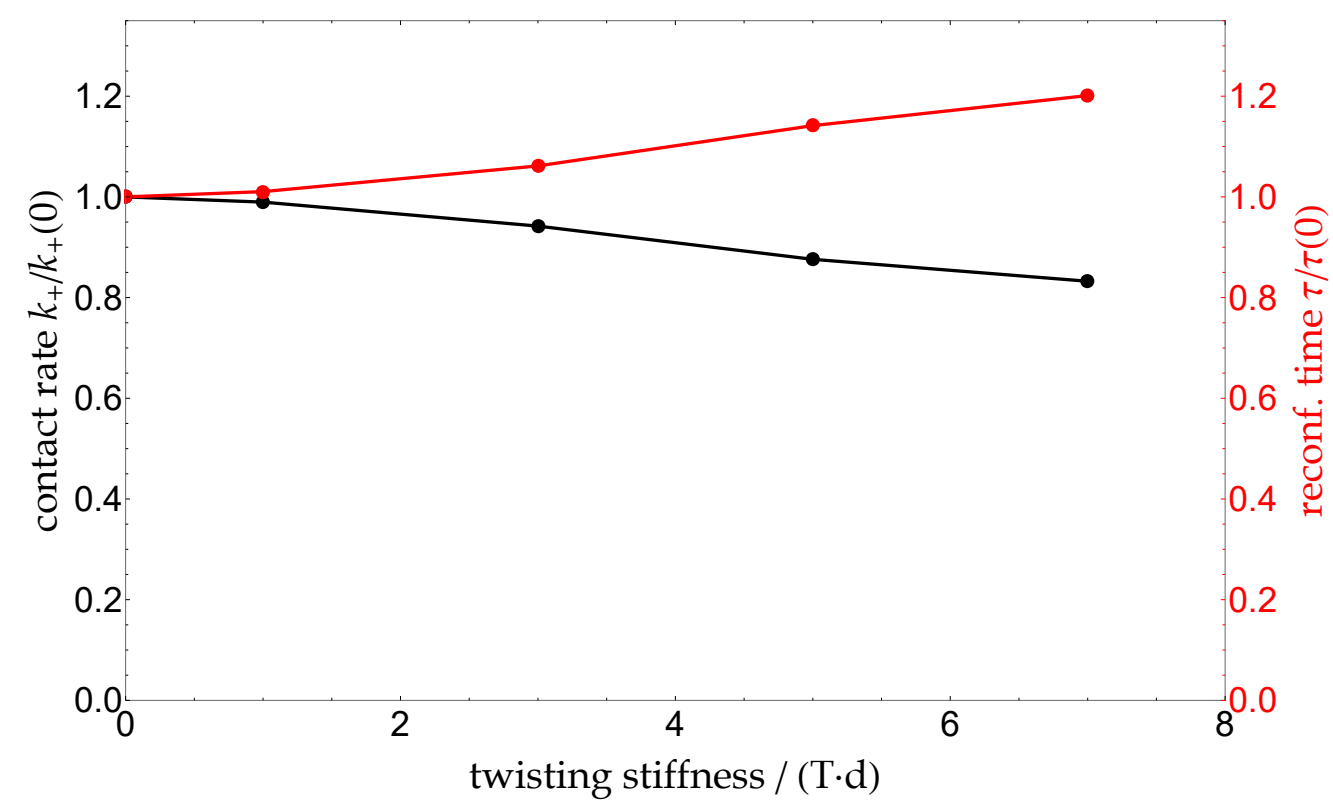

Figure G.1: The simulated end-to-end contact rate (black) and end-to-end reconfiguration time (red) in presence of twisting stiffness.

Reference curvature Next we turn on the reference curvatures $\bar{\Omega}$, starting with the arguably easiest non-straight reference shape: a circular arc, which refers to a constant reference curvature. Again, this makes keeping track of the material frame necessary, and thus a choice must be made concerning the value of the twisting stiffness. Turning off thermal fluctuations, the simulated chain must minimize its energy and thus converge towards the circular arc. I have numerically observed that this behavior cannot be observed when $c$ is set to zero - the material frame does 
not converge towards a Bishop frame, with respect to which the reference curvatures are defined. To avoid multiple new fit parameters, we thus consider the limit $c \rightarrow \infty$ in which the chain immediately reduces its twist. In practice, this can be achieved as follows. Starting at zero twist, a numerical time step for the chain's vertices is performed, which results in nonzero (but very small) twist angles $\bar{\Omega}_{i}^{(3)}$. Assuming a separation of time scales between vertex and twist dynamics, the material frames are then allowed to relax to zero (while keeping the chain vertices fixed) by rotating them around their tangent vectors. This relaxation works as follows: Each summand in the twist energy is the square of a twist angle associated with a vertex. The gradient of this summand with respect to the two adjacent material frames' orientations is linear, but of opposite signs. Therefore, the relaxation of all twist angles obeys an "actio-reactio" principle: The total sum of angles between Bishop frames and material frames along the chain is a preserved quantity. Since the energetic minimum the relaxation converges towards is a Bishop frame, the final result of the untwisting dynamics of the material frames is easily constructed: we (i) construct a Bishop frame which coincides with the material frame at one chain end, (ii) calculate the average angle $\Phi$ between this Bishop frame and the material frame, (iii) globally rotate the Bishop frame by the angle $\Phi$ and (iv) the replace the material frame with the rotated Bishop frame.

Following this integration procedure, we simulated the dynamics of chains with the following reference geometry. They consist of $N=20$ beads with $a=0$ and $R_{\mathrm{EV}}=0$ and form two loops of a planar circle. In the reference state, the first and least bead overlap with each other and with the chain's center position, while the bond length equals 1 . This situation corresponds to reference curvatures $\Omega_{i}^{1} \approx 0.65$ and $\Omega_{i}^{(2)}=0$. The bending stiffness was altered between simulations and its values were chosen such that they would correspond to persistence lengths $l_{P} \in\{0,2,5,10,20\}$ in absence of reference curvatures. From each of these 5 simulations, we calculated the internal-toend contact rates $k_{+}(s)$, which are displayed in figure G.2.

For $l_{P}=\sigma=0$, no energy term is present, and we obtain the monotonically decreasing functional form which we know from the main text. For increasing bending stiffness, the functional form changes. The stiffest chain shows the other extreme case: The contact rate increases with arc length because the energetic barrier which must be overcome for loop formation shrinks with loop length. In between these two extreme cases $\left(l_{P}=10\right)$, we observe a local maximum in the middle of the chain.

We conclude that reference structures introduced by the reference curvatures $\bar{\Omega}$ can indeed lead to nontrivial, non-power-law functional forms of $k_{+}(s)$. They are even capable of reproducing experimentally observed local maxima (see figure 7.1, page 155), and are thus worthwhile to investigate in the future. 


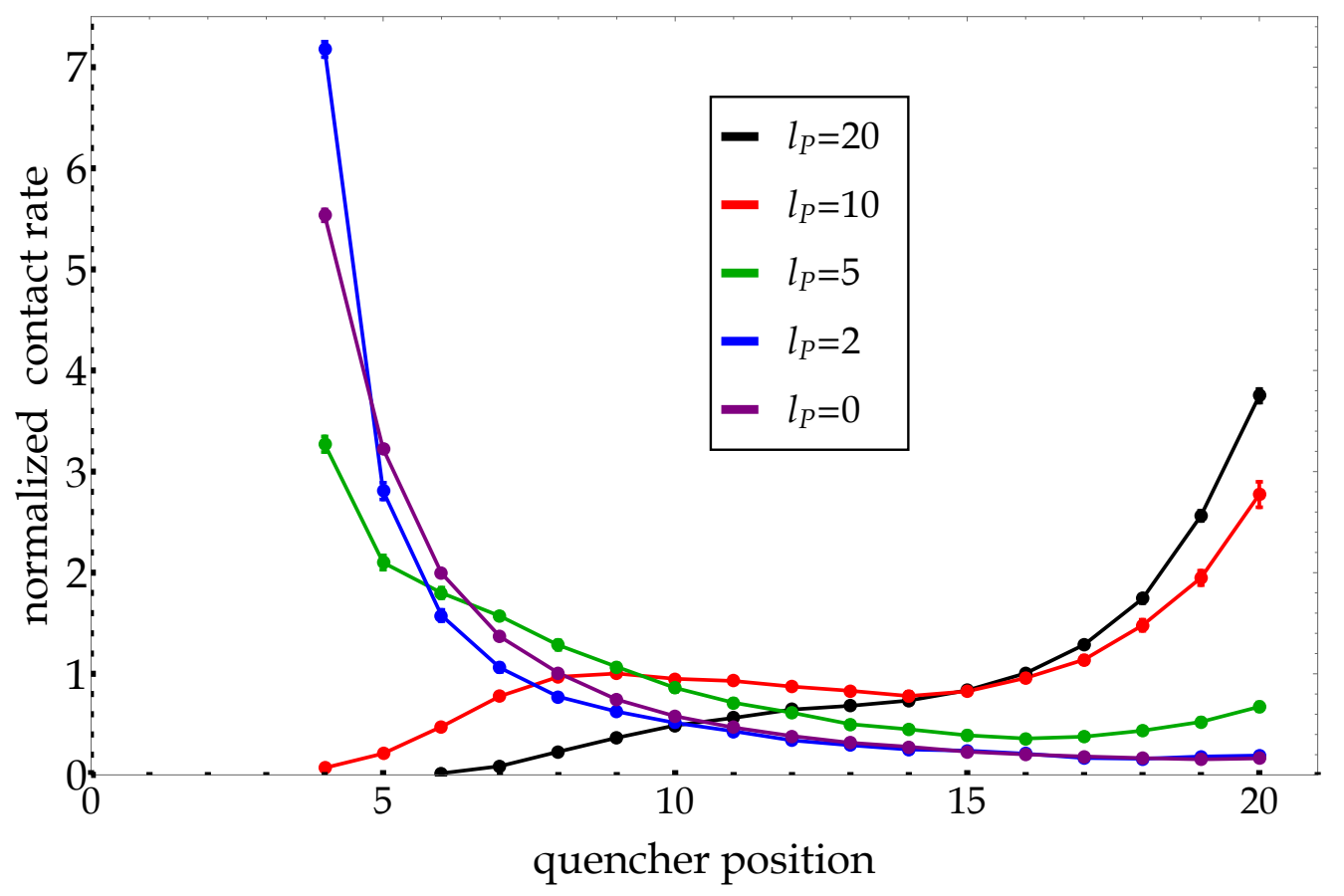

Figure G.2: The simulated internal-to-end contact rate $k_{+}(s)$ in presence of a constant reference curvature which corresponds to two loops of a circular arc. All five curves were individually normalized for visual purposes such that the areas below all of them are identical. 


\section{BIBLIOGRAPHY}

[1] C. Gardiner, Stochastic methods - a handbook for the natural and social sciences, Vol. 4 (Springer Berlin, 2009).

[2] E. Hairer and G. Wanner, Analysis by its history, Vol. 2 (Springer Science \& Business Media, 2006).

[3] H. Neuweiler, M. Löllmann, S. Doose, and M. Sauer, Dynamics of unfolded polypeptide chains in crowded environment studied by fluorescence correlation spectroscopy, Journal of Molecular Biology 365, 856 (2007).

[4] F. Krieger, A. Möglich, and T. Kiefhaber, Effect of proline and glycine residues on dynamics and barriers of loop formation in polypeptide chains, Journal of the American Chemical Society 127, 3346 (2005).

[5] B. Fierz and T. Kiefhaber, End-to-end vs interior loop formation kinetics in unfolded polypeptide chains, Journal of the American Chemical Society 129, 672 (2007).

[6] O. Bieri, J. Wirz, B. Hellrung, M. Schutkowski, M. Drewello, and T. Kiefhaber, The speed limit for protein folding measured by triplet-triplet energy transfer, Proceedings of the National Academy of Sciences 96, 9597 (1999).

[7] M. Buscaglia, L. J. Lapidus, W. A. Eaton, and J. Hofrichter, Effects of denaturants on the dynamics of loop formation in polypeptides, Biophysical Journal 91, 276 (2006).

[8] M. P. Luitz, A. Barth, A. H. Crevenna, R. Bomblies, D. C. Lamb, and M. Zacharias, Covalent dye attachment influences the dynamics and conformational properties of flexible peptides, PLOS ONE 12, e0177139 (2017).

[9] L. J. Lapidus, W. A. Eaton, and J. Hofrichter, Measuring the rate of intramolecular contact formation in polypeptides, Proceedings of the National Academy of Sciences 97, 7220 (2000). 
[10] L. J. Lapidus, P. J. Steinbach, W. A. Eaton, A. Szabo, and J. Hofrichter, Effects of chain stiffness on the dynamics of loop formation in polypeptides, The Journal of Physical Chemistry B 106, 11628 (2002).

[11] R. R. Cheng, T. Uzawa, K. W. Plaxco, and D. E. Makarov, Universality in the timescales of internal loop formation in unfolded proteins and single-stranded oligonucleotides, Biophysical Journal 99, 3959 (2010).

[12] B. Fierz, H. Satzger, C. Root, P. Gilch, W. Zinth, and T. Kiefhaber, Loop formation in unfolded polypeptide chains on the picoseconds to microseconds time scale, Proceedings of the National Academy of Sciences 104, 2163 (2007).

[13] I. Daidone, H. Neuweiler, S. Doose, M. Sauer, and J. C. Smith, Hydrogen-bond driven loop-closure kinetics in unfolded polypeptide chains, PLOS Computational Biology 6 , e1000645 (2010).

[14] F. Krieger, B. Fierz, O. Bieri, M. Drewello, and T. Kiefhaber, Dynamics of unfolded polypeptide chains as model for the earliest steps in protein folding, Journal of Molecular Biology 332, 265 (2003).

[15] P. E. Rouse Jr, A theory of the linear viscoelastic properties of dilute solutions of coiling polymers, The Journal of Chemical Physics 21, 1272 (1953).

[16] B. H. Zimm, Dynamics of polymer molecules in dilute solution: viscoelasticity, flow birefringence and dielectric loss, The Journal of Chemical Physics 24, 269 (1956).

[17] N. Saitô, K. Takahashi, and Y. Yunoki, The statistical mechanical theory of stiff chains, Journal of the Physical Society of Japan 22, 219 (1967).

[18] N. Samanta, J. Ghosh, and R. Chakrabarti, Looping and reconfiguration dynamics of a flexible chain with internal friction, AIP Advances 4, 067102 (2014).

[19] A. Szabo, K. Schulten, and Z. Schulten, First passage time approach to diffusion controlled reactions, The Journal of Chemical Physics 72, 4350 (1980).

[20] S. Jun, J. Bechhoefer, and B.-Y. Ha, Diffusion-limited loop formation of semiflexible polymers: Kramers theory and the intertwined time scales of chain relaxation and closing, EPL (Europhysics Letters) 64, 420 (2003).

[21] G. Wilemski and M. Fixman, Diffusion-controlled intrachain reactions of polymers. I: Theory, The Journal of Chemical Physics 60, 866 (1974). 
[22] G. Wilemski and M. Fixman, Diffusion-controlled intrachain reactions of polymers. II: Results for a pair of terminal reactive groups, The Journal of Chemical Physics 60, 878 (1974).

[23] B. Friedman and B. O'Shaughnessy, Universal behavior in reacting polymer systems, Physical Review Letters 60, 64 (1988).

[24] B. Friedman and B. O'Shaughnessy, Theory of polymer cyclization, Physical Review A 40, 5950 (1989).

[25] B. Friedman and B. O'Shaughnessy, Theory of intramolecular reactions in polymeric liquids, Macromolecules 26, 4888 (1993).

[26] M. Doi, Diffusion-controlled reaction of polymers, Chemical Physics 9, 455 (1975).

[27] A. Dua and B. J. Cherayil, The dynamics of chain closure in semiflexible polymers, The Journal of Chemical Physics 116, 399 (2002).

[28] J. Z. Chen, H.-K. Tsao, and Y.-J. Sheng, Diffusion-controlled first contact of the ends of a polymer: crossover between two scaling regimes, Physical Review E 72, 031804 (2005).

[29] P. Debnath and B. J. Cherayil, Dynamics of chain closure: approximate treatment of nonlocal interactions, The Journal of Chemical Physics 120, 2482 (2004).

[30] N. M. Toan, G. Morrison, C. Hyeon, and D. Thirumalai, Kinetics of loop formation in polymer chains, The Journal of Physical Chemistry B 112, 6094 (2008).

[31] J. J. Portman, Non-Gaussian dynamics from a simulation of a short peptide: loop closure rates and effective diffusion coefficients, The Journal of Chemical Physics 118, 2381 (2003).

[32] F. X.-F. Ye, P. Stinis, and H. Qian, Dynamic looping of a free-draining polymer, SIAM Journal on Applied Mathematics 78, 104 (2018).

[33] R. Chakrabarti, Dynamics of end-to-end loop formation: A flexible chain in the presence of hydrodynamic interaction, Physica A: Statistical Mechanics and its Applications 391, 4081 (2012).

[34] K. Santo and K. Sebastian, Dynamics of loop formation in a semiflexible polymer, Physical Review E 80, 061801 (2009).

[35] A. Dua and B. J. Cherayil, The thermodynamics of reversible cyclization in semiflexible polymers, The Journal of Chemical Physics 117, 7765 (2002). 
[36] G. H. Zerze, J. Mittal, and R. B. Best, Diffusive dynamics of contact formation in disordered polypeptides, Physical Review Letters 116, 068102 (2016).

[37] Z. Wang and D. E. Makarov, Rate of intramolecular contact formation in peptides: the loop length dependence, The Journal of Chemical Physics 117, 4591 (2002).

[38] A. Podtelezhnikov and A. Vologodskii, Simulations of polymer cyclization by Brownian dynamics, Macromolecules 30, 6668 (1997).

[39] R. W. Pastor, R. Zwanzig, and A. Szabo, Diffusion limited first contact of the ends of a polymer: comparison of theory with simulation, The Journal of Chemical Physics 105, 3878 (1996).

[40] R. Afra and B. A. Todd, Kinetics of loop formation in worm-like chain polymers, The Journal of Chemical Physics 138, 174908 (2013).

[41] J. Chen, H.-K. Tsao, and Y.-J. Sheng, First-passage time of cyclization dynamics of a wormlike polymer, EPL (Europhysics Letters) 65, 407 (2004).

[42] S.-H. Chong, P. Chatterjee, and S. Ham, Computer simulations of intrinsically disordered proteins, Annual Review of Physical Chemistry 68, 117 (2017).

[43] S. Pronk, S. Páll, R. Schulz, P. Larsson, P. Bjelkmar, R. Apostolov, M. R. Shirts, J. C. Smith, P. M. Kasson, D. van der Spoel, et al., GROMACS 4.5: a high-throughput and highly parallel open source molecular simulation toolkit, Bioinformatics 29, 845 (2013).

[44] S. A. Hollingsworth and R. O. Dror, Molecular dynamics simulation for all, Neuron 99, 1129 (2018).

[45] C. Cercignani, The Boltzmann equation and its applications (Springer, 1988) pp. 40-103.

[46] E. Moeendarbary, T. Ng, and M. Zangeneh, Dissipative particle dynamics: introduction, methodology and complex fluid applications - a review, International Journal of Applied Mechanics 1, 737 (2009).

[47] G. Gompper, T. Ihle, D. Kroll, and R. Winkler, Multi-particle collision dynamics: A particlebased mesoscale simulation approach to the hydrodynamics of complex fluids (Springer, 2009) pp. 1-87.

[48] T. T. Pham, U. D. Schiller, J. R. Prakash, and B. Dünweg, Implicit and explicit solvent models for the simulation of a single polymer chain in solution: Lattice Boltzmann versus Brownian dynamics, The Journal of Chemical Physics 131, 164114 (2009). 
[49] S. R. Bistafa, On the development of the Navier-Stokes equation by Navier, Revista Brasileira de Ensino de Física 40 (2018).

[50] G. G. Stokes, On the effect of the internal friction of fluids on the motion of pendulums, Vol. 9 (Pitt Press Cambridge, 1851).

[51] C. Oseen, Über die Stoke'sche Formel und über eine verwandte Aufgabe in der Hydrodynamik: Mitteilung 2, Arkiv för matematik, astronomi och fysik No. Bd. 1 (Almqvist \& Wiksell, 1911).

[52] J. Rotne and S. Prager, Variational treatment of hydrodynamic interaction in polymers, The Journal of Chemical Physics 50, 4831 (1969).

[53] H. Yamakawa, Transport properties of polymer chains in dilute solution: hydrodynamic interaction, The Journal of Chemical Physics 53, 436 (1970).

[54] E. Wajnryb, K. A. Mizerski, P. J. Zuk, and P. Szymczak, Generalization of the Rotne-PragerYamakawa mobility and shear disturbance tensors, Journal of Fluid Mechanics $\mathbf{7 3 1}$ (2013).

[55] P. Zuk, E. Wajnryb, K. Mizerski, and P. Szymczak, Rotne-Prager-Yamakawa approximation for different-sized particles in application to macromolecular bead models, Journal of Fluid Mechanics 741 (2014).

[56] D. Flamm, History and outlook of statistical physics, arXiv: physics (1998).

[57] M. V. Smoluchowski, Über Brownsche Molekularbewegung unter Einwirkung äußerer Kräfte und deren Zusammenhang mit der verallgemeinerten Diffusionsgleichung, Annalen der Physik 353, 1103 (1916).

[58] P. Langevin, Sur la théorie du mouvement brownien, Compt. Rendus 146, 530 (1908).

[59] A. D. Fokker, Over Brown'sche Bewegingen in het Stralingsveld, en WaarschijnlijkheidsBeschouw ingenin de Stralingstheorie, Annalen der Physik 43, 812 (1914).

[60] M. Planck, Über einen Satz der statistischen Dynamik und seine Erweiterung in der Quantentheorie, Sitzungsberichte der Preussischen Akademie der Wissenschaften zu Berlin 24 (1917).

[61] A. Einstein, Über die von der molekularkinetischen Theorie der Wärme geforderte Bewegung von in ruhenden Flüssigkeiten suspendierten Teilchen, Annalen der Physik 322, 549 (1905). 
[62] D. L. Ermak and J. McCammon, Brownian dynamics with hydrodynamic interactions, The Journal of Chemical Physics 69, 1352 (1978).

[63] G. T. Barkema and J. Van Leeuwen, Efficient simulation of semiflexible polymers with stiff bonds, Physical Review E 95, 012502 (2017).

[64] H. A. Kramers, The behavior of macromolecules in inhomogeneous flow, The Journal of Chemical Physics 14, 415 (1946).

[65] M. Fixman, Simulation of polymer dynamics. I. General theory, The Journal of Chemical Physics 69, 1527 (1978).

[66] M. Pasquali and D. C. Morse, An efficient algorithm for metric correction forces in simulations of linear polymers with constrained bond lengths, The Journal of Chemical Physics 116, 1834 (2002).

[67] N. Metropolis, A. W. Rosenbluth, M. N. Rosenbluth, A. H. Teller, and E. Teller, Equation of state calculations by fast computing machines, The Journal of Chemical Physics 21, 1087 (1953).

[68] O. Kratky and G. Porod, Röntgenuntersuchung gelöster Fadenmoleküle, Recueil des Travaux Chimiques des Pays-Bas 68, 1106 (1949).

[69] P. J. Flory, Principles of polymer chemistry (Cornell University Press, 1953).

[70] B. S. Khatri and T. C. McLeish, Rouse model with internal friction: A coarse grained framework for single biopolymer dynamics, Macromolecules 40, 6770 (2007).

[71] H. Lopez and V. Lobaskin, Coarse-grained model of adsorption of blood plasma proteins onto nanoparticles, The Journal of Chemical Physics 143, 243138 (2015).

[72] A. Ghavami, E. van der Giessen, and P. R. Onck, Coarse-grained potentials for local interactions in unfolded proteins, Journal of Chemical Theory and Computation $\mathbf{9}, 432$ (2012).

[73] G. L. Dignon, W. Zheng, Y. C. Kim, R. B. Best, and J. Mittal, Sequence determinants of protein phase behavior from a coarse-grained model, PLOS Computational Biology 14, e1005941 (2018).

[74] S. M. Han, H. Benaroya, and T. Wei, Dynamics of transversely vibrating beams using four engineering theories, Journal of Sound and Vibration 225, 935 (1999).

[75] G. Kirchhoff, Über das Gleichgewicht und die Bewegung eines unendlich dünnen elastischen Stabes, Journal für die reine und angewandte Mathematik 56, 285 (1859). 
[76] R. L. Bishop, There is more than one way to frame a curve, The American Mathematical Monthly 82, 246 (1975).

[77] J. Langer and D. A. Singer, Lagrangian aspects of the Kirchhoff elastic rod, SIAM Review 38, 605 (1996).

[78] R. E. Goldstein, T. R. Powers, and C. H. Wiggins, Viscous nonlinear dynamics of twist and writhe, Physical Review Letters 80, 5232 (1998).

[79] M. Bergou, B. Audoly, E. Vouga, M. Wardetzky, and E. Grinspun, Discrete viscous threads, ACM Transactions on Graphics (TOG) 29 (2010).

[80] T. Hirschfeld, Optical microscopic observation of single small molecules, Applied Optics 15, 2965 (1976).

[81] T. Hirschfeld, Quantum efficiency independence of the time integrated emission from a fluorescent molecule, Applied Optics 15, 3135 (1976).

[82] N. J. Dovichi, J. C. Martin, J. H. Jett, and R. A. Keller, Attogram detection limit for aqueous dye samples by laser-induced fluorescence, Science 219, 845 (1983).

[83] E. B. Shera, N. K. Seitzinger, L. M. Davis, R. A. Keller, and S. A. Soper, Detection of single fluorescent molecules, Chemical Physics Letters 174, 553 (1990).

[84] D. Magde, E. L. Elson, and W. W. Webb, Fluorescence correlation spectroscopy. II. An experimental realization, Biopolymers: Original Research on Biomolecules 13, 29 (1974).

[85] J. Widengren and Ü. Mets, Single-Molecule Detection in Solution-Methods and Applications (Wiley-VCH, 2002).

[86] N. Petersen, Scanning fluorescence correlation spectroscopy. I. Theory and simulation of aggregation measurements, Biophysical Journal 49, 809 (1986).

[87] T. Dertinger, V. Pacheco, I. von der Hocht, R. Hartmann, I. Gregor, and J. Enderlein, Two-focus fluorescence correlation spectroscopy: A new tool for accurate and absolute diffusion measurements, ChemPhysChem 8, 433 (2007).

[88] J. K. Dhont, An introduction to dynamics of colloids, Vol. 2 (Elsevier, 1996).

[89] E. M. Purcell, Life at low Reynolds number, American Journal of Physics 45, 3 (1977).

[90] J. Burgers, Second report on viscosity and plasticity, Nordemann, New York, 113 (1938).

[91] M. Lisicki, Four approaches to hydrodynamic Green's functions - the Oseen tensors, arXiv preprint: 1312.6231 (2013). 
[92] I. Oppenheim and N. van Kampen, The kinetic origin of the stick boundary condition, Physica A: Statistical Mechanics and its Applications 122, 277 (1983).

[93] J. Blake, A note on the image system for a Stokeslet in a no-slip boundary, Mathematical Proceedings of the Cambridge Philosophical Society 70 (1971).

[94] R. Singh and R. Adhikari, Fluctuating hydrodynamics and the Brownian motion of an active colloid near a wall, European Journal of Computational Mechanics 26, 78 (2017).

[95] Y. von Hansen, M. Hinczewski, and R. R. Netz, Hydrodynamic screening near planar boundaries: Effects on semiflexible polymer dynamics, The Journal of Chemical Physics 134, 06B609 (2011).

[96] N. Hoda and S. Kumar, Brownian dynamics simulations of polyelectrolyte adsorption in shear flow with hydrodynamic interaction, The Journal of Chemical Physics 127, 234902 (2007).

[97] H. Risken, The Fokker-Planck equation (Springer, 1996).

[98] N. G. Van Kampen, Stochastic processes in physics and chemistry, Vol. 1 (Elsevier, 1992).

[99] R. M. Mazo, Brownian motion: fluctuations, dynamics, and applications (Oxford University Press on Demand, 2002).

[100] J. Garcia-Palacios, Introduction to the theory of stochastic processes and Brownian motion problems, arXiv preprint: condensed matter/0701242 (2007).

[101] G. Schön, Moderne Theoretische Physik III - Statistische Mechanik, Lecture Script - Karlsruher Institut für Technologie (2007).

[102] C. E. Shannon, A mathematical theory of communication, Vol. 27 (Wiley Online Library, 1948) pp. 379-423.

[103] A. Caticha, Lectures on probability, entropy, and statistical physics, arXiv preprint:0808.0012 (2008).

[104] R. Pawula, Approximation of the linear Boltzmann equation by the Fokker-Plank equation, Phys. Rev. 162, 196 (1967).

[105] M. R. R. Tabar, Analysis and data-based reconstruction of complex nonlinear dynamical systems: Stochastic processes with jumps and non-vanishing higher-order KramersMoyal coefficients (Springer, 2019) pp. 99-110.

[106] J. Enderlein, Theoretical biophysics, Lecture Script - Georg-August-Universität Göttingen (2014). 
[107] S. A. V. Gihman I. F., The theory of stochastic processes I, Grundlagen der Mathematischen Wissenschaften 210 (1974).

[108] I. Sokolov, Ito, Stratonovich, Hänggi and all the rest: The thermodynamics of interpretation, Chemical Physics 375, 359 (2010).

[109] N. Van Kampen, Itô versus Stratonovich, Journal of Statistical Physics 24, 175 (1981).

[110] A. Montesi, D. C. Morse, and M. Pasquali, Brownian dynamics algorithm for bead-rod semiflexible chain with anisotropic friction, The Journal of Chemical Physics 122, 084903 (2005).

[111] L. D. Landau and E. M. Lifshitz, Course of Theoretical Physics Vol 7: Theory and Elasticity (Pergamon press, 1959).

[112] R. E. Goldstein and S. A. Langer, Nonlinear dynamics of stiff polymers, Physical Review Letters 75, 1094 (1995).

[113] M. Bergou, M. Wardetzky, S. Robinson, B. Audoly, and E. Grinspun, Discrete elastic rods, ACM transactions on graphics (TOG) 27 (2008).

[114] K. Crane and M. Wardetzky, A glimpse into discrete differential geometry, Notices of the American Mathematical Society 64, 1153 (2017).

[115] M. Doi and S. F. Edwards, The theory of polymer dynamics, Vol. 73 (Oxford University Press, 1988).

[116] P.-G. De Gennes and P.-G. Gennes, Scaling concepts in polymer physics (Cornell University Press, 1979).

[117] A. C. Vaiana, H. Neuweiler, A. Schulz, J. Wolfrum, M. Sauer, and J. C. Smith, Fluorescence quenching of dyes by tryptophan: interactions at atomic detail from combination of experiment and computer simulation, Journal of the American Chemical Society 125, 14564 (2003).

[118] D. Zhong, S. K. Pal, C. Wan, and A. H. Zewail, Femtosecond dynamics of a drug-protein complex: daunomycin with apo riboflavin-binding protein, Proceedings of the National Academy of Sciences 98, 11873 (2001).

[119] X. Li, R. Zhu, A. Yu, and X. S. Zhao, Ultrafast photoinduced electron transfer between tetramethylrhodamine and guanosine in aqueous solution, The Journal of Physical Chemistry B 115, 6265 (2011). 
[120] S. Doose, H. Neuweiler, and M. Sauer, A close look at fluorescence quenching of organic dyes by tryptophan, ChemPhysChem 6, 2277 (2005).

[121] N. Karedla, A. I. Chizhik, I. Gregor, A. M. Chizhik, O. Schulz, and J. Enderlein, Singlemolecule metal-induced energy transfer (smMIET): Resolving nanometer distances at the single-molecule level, ChemPhysChem 15, 705 (2014).

[122] N. Karedla, Single-molecule metal-induced energy transfer: from basics to applications (Springer, 2017).

[123] N. Karedla, A. M. Chizhik, S. C. Stein, D. Ruhlandt, I. Gregor, A. I. Chizhik, and J. Enderlein, Three-dimensional single-molecule localization with nanometer accuracy using Metal-Induced Energy Transfer (MIET) imaging, The Journal of Chemical Physics 148, 204201 (2018).

[124] "Atto-Tec dye properties," https://www.atto-tec.com/fileadmin/user_upload/ Katalog_Flyer_Support/Dye_Properties_01.pd, accessed: 2018-08-01.

[125] E. Buckingham, On physically similar systems; illustrations of the use of dimensional equations, Physical Review 4, 345 (1914).

[126] N. Metropolis, A. W. Rosenbluth, M. N. Rosenbluth, A. H. Teller, and E. Teller, Equation of state calculations by fast computing machines, The Journal of Chemical Physics 21, 1087 (1953).

[127] J. G. de la Torre and V. A. Bloomfield, Hydrodynamic properties of macromolecular complexes. I. Translation, Biopolymers: Original Research on Biomolecules 16, 1747 (1977).

[128] J. G. de la Torre and V. A. Bloomfield, Hydrodynamic properties of complex, rigid, biological macromolecules: theory and applications, Quarterly Reviews of Biophysics 14, 81 (1981).

[129] S. Mühle, M. Zhou, A. Ghosh, and J. Enderlein, Loop formation and translational diffusion of intrinsically disordered proteins, Physical Review E 100, 052405 (2019).

[130] M. Zhou, Study conformational dynamics of intrinsically disordered proteins by singlemolecule spectroscopy (Georg-August-Universität Göttingen, PhD Thesis, 2016).

[131] P. Kapusta, Absolute diffusion coefficients: compilation of reference data for FCS calibration, Application note, PicoQuant GmbH: Berlin (2010).

[132] V. Bloomfield, D. Crothers, and I. Tinoco Jr, Nucleic acids: structures, properties, and functions, Calif: University Science Books 794 (2000). 
[133] G. S. Manning, The persistence length of DNA is reached from the persistence length of its null isomer through an internal electrostatic stretching force, Biophysical Journal 91, 3607 (2006).

[134] A. Y. Sim, J. Lipfert, D. Herschlag, and S. Doniach, Salt dependence of the radius of gyration and flexibility of single-stranded DNA in solution probed by small-angle $x$-ray scattering, Physical Review E 86, 021901 (2012).

[135] B. Tinland, A. Pluen, J. Sturm, and G. Weill, Persistence length of single-stranded DNA, Macromolecules 30, 5763 (1997).

[136] Q. Chi, G. Wang, and J. Jiang, The persistence length and length per base of single-stranded DNA obtained from fluorescence correlation spectroscopy measurements using mean field theory, Physica A: Statistical Mechanics and its Applications 392, 1072 (2013).

[137] D. E. Makarov, Spatiotemporal correlations in denatured proteins: The dependence of fluorescence resonance energy transfer (FRET)-derived protein reconfiguration times on the location of the FRET probes, The Journal of Chemical Physics 132, $01 \mathrm{~B} 612$ (2010).

[138] P. Szymczak and M. Cieplak, Proteins in a shear flow, The Journal of Chemical Physics 127, 10B618 (2007).

[139] E. Banachowicz, J. Gapiński, and A. Patkowski, Solution structure of biopolymers: a new method of constructing a bead model, Biophysical Journal 78, 70 (2000).

[140] J. Antosiewicz and D. Porschke, Volume correction for bead model simulations of rotational friction coefficients of macromolecules, The Journal of Physical Chemistry 93, 5301 (1989).

[141] T. Hellweg, W. Eimer, E. Krahn, K. Schneider, and A. Müller, Hydrodynamic properties of nitrogenase - the MoFe protein from Azotobacter vinelandii studied by dynamic light scattering and hydrodynamic modelling, Biochimica et Biophysica Acta (BBA)-Protein Structure and Molecular Enzymology 1337, 311 (1997).

[142] T. Frembgen-Kesner and A. H. Elcock, Striking effects of hydrodynamic interactions on the simulated diffusion and folding of proteins, Journal of Chemical Theory and Computation 5, 242 (2009).

[143] H. Hofmann, A. Soranno, A. Borgia, K. Gast, D. Nettels, and B. Schuler, Polymer scaling laws of unfolded and intrinsically disordered proteins quantified with single-molecule spectroscopy, Proceedings of the National Academy of Sciences (2012). 
[144] H.-X. Zhou, Polymer models of protein stability, folding, and interactions, Biochemistry 43, 2141 (2004).

[145] A. Soranno, B. Buchli, D. Nettels, R. R. Cheng, S. Müller-Späth, S. H. Pfeil, A. Hoffmann, E. A. Lipman, D. E. Makarov, and B. Schuler, Quantifying internal friction in unfolded and intrinsically disordered proteins with single-molecule spectroscopy, Proceedings of the National Academy of Sciences 109, 17800 (2012).

[146] A. Soranno, A. Holla, F. Dingfelder, D. Nettels, D. E. Makarov, and B. Schuler, Integrated view of internal friction in unfolded proteins from single-molecule FRET, contact quenching, theory, and simulations, Proceedings of the National Academy of Sciences 114, E1833 (2017).

[147] "Atomic weights and isotopic compositions for all elements," https://physics.nist.gov/ cgi-bin/Compositions/stand_alone.pl, accessed: 2020-05-04.

[148] J. P. Müller, S. Mielke, A. Löf, T. Obser, C. Beer, L. K. Bruetzel, D. A. Pippig, W. Vanderlinden, J. Lipfert, R. Schneppenheim, et al., Force sensing by the vascular protein von Willebrand factor is tuned by a strong intermonomer interaction, Proceedings of the National Academy of Sciences 113, 1208 (2016).

[149] D. C. Morse, Theory of constrained Brownian motion, Advances in Chemical Physics 128, 65 (2004).

[150] E. Hinch, Brownian motion with stiff bonds and rigid constraints, Journal of Fluid Mechanics 271, 219 (1994).

[151] B. Delmotte, E. Climent, and F. Plouraboué, A general formulation of bead models applied to flexible fibers and active filaments at low Reynolds number, Journal of Computational Physics 286, 14 (2015).

[152] H. B. Callen and T. A. Welton, Irreversibility and generalized noise, Physical Review 83, 34 (1951).

[153] N. Berova, K. Nakanishi, and R. W. Woody, Circular dichroism: principles and applications (John Wiley \& Sons, 2000).

[154] G. N. Ramachandran, Stereochemistry of polypeptide chain configurations, Journal of Molecular Biology 7, 95 (1963).

[155] B. Cappella and G. Dietler, Force-distance curves by atomic force microscopy, Surface science reports 34, 1 (1999). 
[156] N. Molkenthin, S. Mühle, A. S. Mey, and M. Timme, Self-organized emergence of folded protein-like network structures from geometric constraints, PLOS ONE 15, e0229230 (2020).

[157] S. Chandrasekhar and W. Reid, On the expansion of functions which satisfy four boundary conditions, Proceedings of the National Academy of Sciences of the United States of America 43, 521 (1957).

[158] A. Hilfinger and F. Jülicher, The chirality of ciliary beats, Physical Biology 5 (2008).

[159] D. Ruhlandt, S. Mühle, and J. Enderlein, Electric field lines of relativistically moving point charges, American Journal of Physics 88, 5 (2020).

[160] L. Landau and E. Lifshitz, The classical theory of fields (Pergamon, 1971) Chap. 3, pp. 1-10.

[161] J. Jackson, Classical electrodynamics (John Wiley \& Sons, 1998) Chap. 14, pp. 661-707.

[162] J. R. Tessman and J. T. Finnell Jr, Electric field of an accelerating charge, American Journal of Physics 35, 523 (1967).

[163] R. Y. Tsien, Pictures of dynamic electric fields, American Journal of Physics 40, 46 (1972).

[164] H. C. Ohanian, Electromagnetic radiation fields: A simple approach via field lines, American Journal of Physics 48, 170 (1980).

[165] O. D. Jefimenko, Direct calculation of the electric and magnetic fields of an electric point charge moving with constant velocity, American Journal of Physics 62, 79 (1994).

[166] B. Bolotovskii and A. Serov, On the force line representation of radiation fields, PhysicsUspekhi 40, 1055 (1997).

[167] A. K. Singal, A first principles derivation of the electromagnetic fields of a point charge in arbitrary motion, American Journal of Physics 79, 1036 (2011).

[168] S. Datta, Visualizing electromagnetic fields using Gnuplot. Part 2: field from accelerating charged particle, Physics Education 32 (2016).

[169] J. Franklin and A. Ryder, Electromagnetic field visualization in virtual reality, American Journal of Physics 87, 153 (2019).

[170] E. M. Purcell and D. J. Morin, Electricity and magnetism (Cambridge University Press, 2013) Chap. 5.7, pp. 251-255. 
[171] R. Satija, A. Das, S. Mühle, J. Enderlein, and D. E. Makarov, Kinetics of loop closure in disordered proteins: theory vs simulations vs experiments, The Journal of Physical Chemistry B (2020).

[172] B. Hamprecht and H. Kleinert, End-to-end distribution function of stiff polymers for all persistence lengths, Physical Review E 71, 031803 (2005). 


\section{ACKNOWLEDGements}

Der meiste Dank geht natürlich an meine Eltern Berit und Werner, die mir ein Leben ermöglicht haben, in dem ich mich jahrelang sorgenfrei auf mein Studium konzentrieren konnte. Dass das keine Selbstverständlichkeit ist, weiß ich zu schätzen.

Furthermore, I would like to thank:

Liz, my wife, editor and semi-quarantine partner. You're my favourite aspect of our branch of the wavefunction (come on, what were the odds?!) and thus my main reason against time travel. Trading that salami for some cheese was easily the best deal I've ever made.

Jörg Enderlein for providing me (in the very sense of this thesis' opening quote) with "the freedom to make my own mistakes". I could have never developed my polymer model if you had pushed me towards a quick publication of the GS-project. With all honesty, spending the first two years of my $\mathrm{PhD}$ running into dead ends without producing publishable results was the best thing I could have done. Your pressure-free policy of open doors and discussions on eye-level really worked for me!

Max Wardetzky for setting me on the right track early on: Having you on my TAC was a great choice, proving the usefulness of TACs in the first place. Without your advice, I may have never learned about discrete differential geometry and probably would have ended up failing to introduce stochastic terms into a continuous framework of elasticity. I'm glad you took an interest in my work - it always excited me to be able to discuss science eye to eye with math professor(s) as a deputy of physics.

Matthias Häring for proofreading parts of this thesis and providing valuable feedback about the foundations of statistical mechanics. Thank you also for taking the initiative on reading the Strogatz book during our 5th semester, writing our Bachelor thesis' in the HZI, doing our Master in Göttingen and getting into stochastic thermodynamics. Those were great influences in my (scientific) life, and I sincerely doubt I'd have found this path on my own. 
Simon Christoph Stein for valuable discussions and advice, especially in the early stage of my $\mathrm{PhD}$, but also concerning this thesis. Multiple times, you have made me take a step back and see things from a different perspective, which is really what it's all about, isn't it? Thank you also for setting up the code to run simulations on the GWDG cluster, which I still use today. Oh, and for lighting a bonfire with a gas cooker - we did it!

Jan Thiart for proofreading parts of this thesis and helping me get started as a PhD student. I'm deeply impressed by your ability to provide valuable feedback at all the levels - language, formatting, but also content.

Morten Wesche for proofreading parts of this thesis and letting me copy your homework in the "Mathematical English" course - the beginning of a refreshingly nerdy friendship.

Jan Mudler for proofreading parts of this thesis and being a loyal friend who made me tea in the Scottish highlands. Good times!

All my colleagues, in particular Arindam Ghosh, Man Zhou, Sebastian Isbaner, Soheil Mojiri, Jan Christoph Thiele, Hongje Jang, Tao Chen, Ingo Gregor and Roman Tsukanov. You have created a productive and fun working environment amongst friends rather than just colleagues. What better job is there?

Dmitrii Makarov, Ben Schuler, Piotr Szymczak and Hagen Hofmann for answering my many emails with questions about polymer science, and thereby helping to set me on the right track. This makes me believe that our scientific community is friendly and supportive rather than competitive.

GGNB-PBCS and the Third Physical Institute, in particular Frauke Bergmann, Sabine Huhnold, Elke Zech, Regina Wunderlich and Thomas Geiling for creating a healthy working atmosphere in which I never felt left alone with non-scientific problems. 